\title{
Phase I Focused Corrective Measures Study/Feasibility Study for the L-Area Oil and Chemical Basin (904-83G) Rev. 0
}

by

E. Palmer

Westinghouse Savannah River Company

Savannah River Site

Aiken, South Carolina 29808

This paper was prepared in connection with work done under the above contract number with the U.S. Department of Energy. By acceptance of this paper, the publisher and/or recipient acknowledges the U. S. Government's right to retain a nonexclusive, royalty-free license in and to any copyright covering this paper, along with the right to reproduce and to authorize others to reproduce all or part of the copyrighted paper. 


\section{DISCLAIMER}

This report was prepared as an account of work sponsored by an agency of the United States Government. Neither the United States Government nor any agency thereof, nor any of their employees, makes any warranty, express or implied, or assumes any legal liability or responsibility for the accuracy, completeness, or usefulness of any information, apparatus, product, or process disclosed, or represents that its use would not infringe privately owned rights. Reference herein to any specific commercial product, process, or service by trade name, trademark, manufacturer, or otherwise does not necessarily constitute or imply its endorsement, recommendation, or favoring by the United States Government or any agency thereof. The views and opinions of authors expressed herein do not necessarily state or reflect those of the United States Government or any agency thereof.

This report has been reproduced directly from the best available copy.

Available to DOE and DOE contractors from the Office of Scientific and Technical Information, P. O. Box 62, Oak Ridge, TN 37831; prices available from (423) 576-8401.

Available to the public from the National Technical Information Service, U. S. Department of Commerce, 5285 Port Royal Road, Springfield, VA 22161. 


\section{DISCLAIMER}

Portions of this document may be illegible electronic image products. Images are produced from the best available original document. 


\section{PHASE I \\ FOCUSED CORRECTIVE MEASURES STUDY/ FEASIBLITY STUDY}

FOR THE

\section{L-AREA OIL AND CHEMICAL BASIN 904-83G (U)}

FINAL

\section{FEBRUARY 1997}

Westinghouse Savannah River Company Savannah River Site Aiken, South Carolina 29808

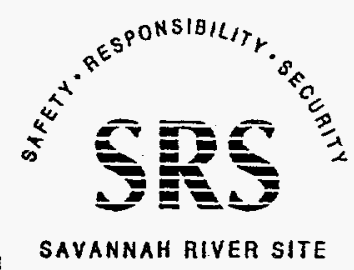

PREPARED FOR THE U.S. DEPARTMENT OF ENERGY UNDER CONTRACT DE-AC09-96-SR18500 
THIS PAGE INTENTIONALLY LEFT BLANK 


\section{PHASE I FOCUSED CMS/FS REPORT L-AREA OIL AND CHEMICAL BASIN 904-83G (U)}

\section{DISCLAIMER}

This report was prepared for the United States Department of Energy under Contract No. DE-AC09-96-SR18500 and is an account of work performed under that contract. Reference herein to any specific commercial product, process, or service by trademark, name, manufacturer or otherwise does not necessarily constitute or imply endorsement, recommendation, or favoring of same by Westinghouse Savannah River Company or by the United States Government or any agency thereof. The views and opinions of the authors expressed herein do not necessarily state or reflect those of the United States Government or any agency thereof.

Printed in the United States of America

Prepared For

U.S. Department of Energy

and

Westinghouse Savannah River Company

Prepared by

Rust Environment \& Infrastructure Inc.

Aiken, South Carolina 
THIS PAGE INTENTIONALLY LEFT BLANK 


\section{CERTIFICATION}

\section{Focused Corrective Measures Study/Feasibility Study (CMS/FS) for the L-Area Oil, \& Chemical Basin and the L-Area Acid/Caustic Basin Rev. 1.1 (U)}

"I certify under the penalty of law that this document and all attachments were prepared under my direction or supervision in accordance with a system designed to assure that qualified personnel properly gather and evaluate the information submitted. Based on my inquiry of the person or persons who manage the system, the information submitted is, to the best of my knowledge and belief, true, accurate, and complete. I am aware that there are significant penalties for submitting false information, including the possibility of fines and imprisonment for knowing violations."

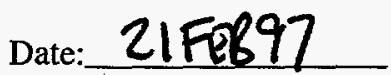

Date: $\operatorname{Heb} 27,1997$
Signature:

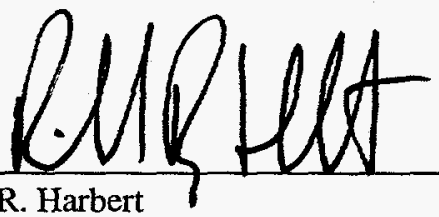

R. R. Harbert

Vice President and General Manager

Environmental Restoration Division

Westinghouse Savannah River Company

Co-operator for the U.S. Dept. of Energy

Savanaah River Operations

Signature:

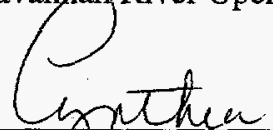

C. V.Apderson

Director

Environmental Restoration Division

U. S. Department of Energy

Savannah River Field Office

Owner and Co-operator 


\section{TABLE OF CONTENTS}

$\underline{\text { Page }}$

EXECUTIVE SUMMARY

ES-1

1.0 INTRODUCTION

$1.1 \quad$ Purpose

1.1.1 SRS RCRA 3004(u)/CERCLA Program Description

1.2 Approach and Report Organization

1.3 Background Information

1.3.1 Unit Description

1.3.1.1 Surface Features and Topography

1.3.1.2 Ecology

1.3.1.3 Meteorology

1.3.1.4 Geology

$1-10$

1.3.1.5 Soil

$1-13$

1.3.1.6 Hydrogeology

$1-13$

1.3.1.7 Surface Water Hydrology

1.3.2 Unit History

1.3.3 Conceptual Unit Model

1.3.4 Nature and Extent of Contamination

$1-18$

1.3.4.1 Summary of Nature and Extent of Contamination in the LAOCB

1.3.4.2 Conclusions Regarding Nature and Extent of Contamination

1.3.5 Contaminant Fate and Transport

1.3.5.1 Chemical Persistence

1.3.5.2 Chemical Migration

1.3.6 Summary of the Risk Assessment $\quad 1-31$

1.3.6.1 Human Health Risk Assessment $\quad 1-32$

1.3.6.2 Ecological Risk Assessment 1-33

1.3.6.3 Risk Summary 1-36 


\subsection{TECHNOLOGY IDENTIFICATION AND SCREENING}

$2.1 \quad$ Introduction

2.2 Remedial Action Objectives

2.2.1 Applicable or Relevant and Appropriate Requirements (ARARs) 2-3

2.2.1.1 Definition of ARARs

2.2.1.2 To-Be-Considered Information

2.2.1.3 Types of ARARs

2.2.2 Remedial Action Objectives for LAOCB and LAOCB Pipeline Soil 2-6

2.2.2.1 Identification of Chemical-Specific ARARs 2-7

2.2.2.2 To-Be-Considered Information 2-9

2.2.2.3 Summary of Remedial Action Objectives for Soil 2-13

2.2.3 Remedial Action Objectives for LAOCB Pipelines - 2-15

2.2.4 Remedial Action Objectives for Vegetation 2-16

2.3 General Response Actions : 2-16

2.3.1 General Response Actions for Soil 2-17

2.3.2 Estimated Volume of Impacted Soil 2-17

2.3.3 General Response Actions for Pipelines 2-18

2.3.4 Estimated Volume of Impacted Pipeline 2-18

$\begin{array}{lll}2.4 & \text { Listing of Technologies } & 2-19\end{array}$

$\begin{array}{ll}2.5 & \text { Screening Of Technologies }\end{array}$

2.5.1 Screening Criteria $2-21$

2.5.1.1 Effectiveness 2-21

2.5.1.2 Implementability 2-22

2.5.1.3 Cost $2-22$

2.5.2 Description and Evaluation of LAOCB Soil Technologies 2-23

2.5.2.1 No Action 2-23

2.5.2.2 Institutional Controls $2-24$

2.5.2.3 Containment $2-25$

2.5.2.4 In-Situ Treatment $\quad 2-29$

2.5.2.5 Ex-Situ Treatment 2-36

2.5.2.6 Disposal of LAOCB Soil and the LAOCB Pipeline 2-44

2.5.2.7 Technology Screening Summary _ - $2-48$ 
3.0 DEVELOPMENT AND EVALUATION OF REMEDIAL 3-1 ALTERNATIVES

3.1 Development and Evaluation of Remedial Alternatives for LAOCB Soil 3-1

3.1.1 Development of Remedial Alternatives 3-2

3.1.2 Screening of Remedial Alternatives 3-4

3.1.2.1 Alternatives Under the General Response of No Action 3-7

3.1.2.2 Alternatives Under the General Response of Containment 3-10

3.1.2.3 Alternatives Under the General Response of In-Situ 3-15 Treatment

3.1.2.4 Alternatives Under the General Response of Ex-Situ 3-23 Treatment

3.1.2.5 Alternatives Under the General Response of 3-32 Off-Unit Disposal

3.1.2.6 Summary of LAOCB Alternative Screening 3-37

3.2 Development and Evaluation of Remedial Alternatives for 3-38 LAOCB Pipelines

3.2.1 Development of Remedial Alternatives 3-38

3.2.2 Screening of Remedial Alternatives $\quad 3-40$

3.2.2.1 Alternatives Under the General Response of No Action 3-41

3.2.2.2 Alternatives Under the General Response of 3-43 Institutional/Administrative Controls

3.2.2.3 Alternatives Under the General Response of Containment 3-44

3.2.2.4 Alternatives Under the General Response of In-Situ 3-46 Treatment

3.2.2.5 Alternatives Under the General Response of Disposal 3-49

3.2.2.6 Summary of LAOCB Pipeline Alternatives Screening 3-53

4.0 DETAILED ANALYSIS OF ALTERNATIVES 4-1

4.1 Introduction $4-1$

4.1.1 Threshold Criteria $4-2$

4.1.2 Primary Balancing Criteria 4-3

4.1.3 Primary Modifying Criteria 4-4

4.2 Individual Analysis of Soil Alternatives _ - 4-5 
4.2.1 Alternative S-1: No Action 4-5

4.2.2 Alternative S-2: Capping : 4-10

4.2.3 Alternative S-3: Slurry Cut-Off Wall and Capping 4-16

4.2.4 Alternative S-4: In-Situ Stabilization and Capping 4-21

4.2.5 Alternative S-5: Ex-Situ Stabilization and Capping 4-26

4.2.6 Alternative S-6: Excavation and Disposal at the NTS Facility 4-31

4.2.7 Comparative Analysis 4-35

4.2.7.1 Overall Protection of Human Health and the Environment 4-35

4.2.7.2 Compliance with ARARs 4-36

4.2.7.3 Long-Term Effectiveness and Permanence 4-37

4.2.7.4 Reduction of Toxicity, Mobility, or Volume 4-38

4.2.7.5 Short-Term Effectiveness $\quad$ 4-39

4.2.7.6 Implementability 4-40

4.2.7.7 Cost $\quad 4-41$

4.3 Individual Analysis of Pipeline Alternatives

4.3.1 Alternative P-1: No Action 4-42

4.3.2 Alternative P-2: Capping 4-45

4.3.3 Alternative P-3: In-situ S/S, Excavation and Disposal in the LAOCB $\quad$ 4-49

4.3.4 Alternative P-4: Excavation and Disposal at the NTS Facility 4-52

4.3.5 Comparative Analysis $\quad$ 4-56

4.3.5.1 Overall Protection of Human Health and the Environment 4-56

4.3.5.2 Compliance with ARARs $\quad$ 4-57

4.3.5.3 Long-Term Effectiveness and Permanence 4-58

4.3.5.4 Reduction of Toxicity, Mobility, or Volume 4-58

4.3.5.5 Short-Term Effectiveness 4-59

$\begin{array}{ll}4.3 .5 .6 & \text { Implementability } \quad 4-60\end{array}$

$\begin{array}{lll}4.3 .5 .7 & \text { Cost } & 4-60\end{array}$

REFERENCES

REF-1

\section{APPENDICES}

Appendix A Dose Rate Calculations

Appendix B Cost Estimates 


\section{LIST OF FIGURES}

Figure 1-1 Overall Feasibility Study/Corrective Measures Study Process

Figure 1-2 Location of the Savannah River Site (SRS) and Major SRS

Facilities

Figure 1-3 Topographic Map of the L-Area Oil and Chemical Basin and Surrounding Area

Figure 1-4 L-Area Oil and Chemical Basin and Vicinity

Figure 1-5 Gross Alpha Concentration Versus Depth

Figure 1-6 Gross Beta Concentration Versus Depth

Figure 1-7a Conceptual Unit Model

Figure 1-7b Conceptual Unit Model

Figure 1-7c Conceptual Unit Model

Figure 1-7d Conceptual Unit Model

Figure 1-7e Conceptual Unit Model

Figure 1-7f Conceptual Unit Model

Figure 1-7g Conceptual Unit Model

Figure 1-7h Conceptual Unit Model

Figure 2-1 Response Action Selection Process

Figure 2-2 In-Situ (Deep) Grouting Techniques

Figure 2-3 Terra-Vit Melter Ex-Situ Vitrification

Figure 3-1a Reduction in Cesium-137 and Cobalt-60 Concentration and

Corresponding Annual Effective Dose Rate through Radioactive Decay

Figure 3-1b Annual Effective Dose Rate Reduction through Capping

Figure 3-2a Proposed Capping System and Slurry Cut-Off Wall Configuration 


\section{LIST OF FIGURES (CONTINUED)}

Figure 3-2b Proposed Capping System for the LAOCB and Pipelines

Figure 4-1 Components of the Detailed Analysis of Alternatives

Figure 4-2 Components of the Nine NCP Criteria

Figure 4-3 Components of the Nine CMS Criteria 


\section{LIST OF TABLES}

Table ES-1

Table ES-2

Table ES-3

Table ES-4

Table ES-5

Table 1-1

Table 1-2

Table 1-3

Table 1-4

Table 1-5

Table 1-6

Table 1-7

Table 1-8

Table 2-1

Table 2-2

Table 2-3

Table 2-4
Primary and Secondary COCs and RGOs

ES-11

Summary of Remedial Alternatives Screening LAOCB Soil

ES-12

ES-15

Summary of Remedial Alternatives Screening LAOCB Pipelines

Comparative Analysis of Soil/Sediment Alternatives

ES-16

Comparative Analysis of Pipeline Alternatives

ES-22

Preferred Habitat of Threatened and Endangered Species in Vicinity of L-Area

Summary of Detected Constituents

$1-73$ in Soil

Soil Adjacent to the LAOCB, Summary of Detected Constituents

$1-74$ in Soil

LAOCB Pipeline, Summary of Detected Constituents in Soil

$1-75$

LAOCB Standing Water, Summary of Detected Constituents

$1-76$

Radioactive Isotope Half-Lives

$1-77$

Summary of Human Health Chemicals of Concern

$1-78$

Potential Location Specific ARARs

$2-63$

Estimated Human Health Risk-Based Remedial Goal Options

$2-67$

Listing of Potentially Applicable Remedial Technologies

$2-71$

L-Area Oil and Chemical Basin Soil

Listing of Potentially Applicable Remedial Technologies

2-72 L-Area Oil and Chemical Basin Pipelines 


\section{LIST OF TABLES (CONTINUED)}

Table 2-5

Technology Screening Summary

L-Area Oil and Chemical Basin Soil

Table 2-6

Summary of Retained Technologies

L-Area Oil and Chemical Basin Soil

Table 2-7

Technology Screening Summary

L-Area Oil and Chemical Basin Pipeline

Table 2-8

Summary of Retained Technologies

L-Area Oil and Chemical Basin Pipeline

Table 3-1

Summary of Remedial Alternatives Screening for LAOCB Soil

Table 3-2

Summary of Remedial Alternatives Screening for LAOCB Pipelines

$3-70$

Table 4-1

Comparative Analysis of Soil/Sediment Alternatives

4-73

Table 4-2

Comparative Analysis of Pipeline Alternatives

4-79 


\section{LIST OF ACRONYMS}

ac

$\mathrm{Al}$

Am

ARARs

As

$\mathrm{Ba}$

$\mathrm{B}$

$\mathrm{Be}$

$\mathrm{Bi}$

bls

BRA

BRC

${ }^{0} \mathrm{C}$

$\mathrm{Ca}$

CA

Cd

CERCLA

Ci

$\mathrm{cm}$

CMS

Co

$\mathrm{COC}$

COPCs

Cs

$\mathrm{Cu}$

DOE

DOT

dpm

EPA

$\mathrm{Eu}$

${ }^{0} \mathrm{~F}$

FFA

FS

$\mathrm{ft}$

$\mathrm{g}$

$\mathrm{H}$

ha

HI

HSWA

HQ

IA-ROD acres

aluminum

Americium

applicable or relevant and appropriate requirements

arsenic

barium

boron

beryllium

bismuth

below land surface

Baseline Risk Assessment

below regulatory concern

degrees Celsius

calcium

control area

cadmium

Comprehensive Environmental Response, Compensation, and Liability Act

Curie

centimeter

Corrective Measures Study

cobalt

chemical of concern

chemicals of potential concern

cesium

copper

U.S. Department of Energy

U.S. Department of Transport

disintegrations per minute

U.S. Environmental Protection Agency

Europium

degrees Fahrenheit

Federal Facility Agreement

Feasibility Study

feet

gram

hydrogen $(\mathrm{H}-3=$ tritium $)$

hectares

hazard index

Hazardous and Solid Waste Amendments

hazard quotient

Interim Action - Record of Decision 


\section{LIST OF ACRONYMS (CONTINUED)}

$\begin{array}{ll}\text { in } & \text { inch } \\ \mathrm{K} & \text { potassium } \\ \mathrm{kg} & \text { kilogram } \\ \mathrm{km} & \text { kilometer } \\ \text { LAACB } & \text { L-Area Acid Caustic Basin } \\ \text { LAHS } & \text { L-Area Hot Shop } \\ \text { LAOCB } & \text { L-Area Oil and Chemical Basin } \\ \text { LOAELs } & \text { lowest-observed-adverse-effect levels } \\ \mathrm{m} & \text { meter } \\ \mathrm{MCL} & \text { maximum contaminant level } \\ \mathrm{MEK} & \text { methyl ethyl ketone } \\ \mathrm{mg} & \text { milligram } \\ \mathrm{Mg} & \text { magnesium } \\ \mathrm{mi} & \text { mile } \\ \mathrm{Mn} & \text { manganese } \\ \mathrm{msl} & \text { mean sea level } \\ \mathrm{Na} & \text { sodium } \\ \mathrm{NCP} & \text { National Oil and Hazardous Substances Contingency Plan } \\ \mathrm{NEPA} & \text { National Environmentai Policy Act } \\ \mathrm{NESHAP} & \text { National Emissions Standards for Hazardous Air Pollutants } \\ \mathrm{Ni} & \text { nickel } \\ \mathrm{NPL} & \text { National Priorities List } \\ \mathrm{NTS} & \text { Nevada Test Site } \\ \mathrm{OU} & \text { Operable Unit } \\ \mathrm{OCPD} & \text { octachlorodibenzo-p-dioxin } \\ \mathrm{Pb} & \text { lead } \\ \mathrm{PCBs} & \text { polychlorinated biphenyls } \\ \mathrm{PCE} & \text { tetrachloroethene } \\ \mathrm{pCi} & \text { picoCurie } \\ \mathrm{PIP} & \text { Public Involvement Plan } \\ \mathrm{PP} & \text { Proposed Plan } \\ \mathrm{PRGs} & \text { Preliminary Remedial Goals } \\ \mathrm{Pu} & \text { plutonium } \\ \mathrm{Ra} & \text { radium } \\ \mathrm{RCRA} & \text { Resource Conservation and Recovery Act } \\ \mathrm{RFA} & \text { RCRA Facility Assessment } \\ \mathrm{RFI} & \text { RCRA Facility Investigation } \\ \mathrm{RGO} & \text { remedial goal option } \\ & \text { Remedial Investigation } \\ \text { reasonable maximum exposure } \\ \end{array}$




\section{LIST OF ACRONYMS (CONTINUED)}

\begin{tabular}{ll} 
Rn & radon \\
Ru & ruthenium \\
SARA & Superfund Amendments and Reauthorization Act \\
Sb & antimony \\
SCDHEC & South Carolina Department of Health and Environmental Control \\
SDCF & Soil Debris Consolidation Facility \\
Sr & strontium \\
SRFS & Savannah River Forest Service \\
SRS & Savannah River Site \\
S/S & stabilization/solidification \\
SVOCs & semi-volatile organic compounds \\
SWMUs & Solid Waste Management Units \\
TBC & to-be-considered \\
TCE & trichloroethene \\
TES & Threatened, Endangered, or Sensitive \\
Th & thorium \\
Tl & thallium \\
TPH & total petroleum hydrocarbons \\
TSCA & Toxic Substances Control Act \\
U & uranium \\
UMTRCA & Uranium Mill Tailings Radiation Control Act \\
V & vanadium \\
VOCs & volatile organic compounds \\
WAC & waste acceptance criteria \\
WSRC & Westinghouse Savannah River Company \\
yd & yard \\
Zn & zinc \\
\hline
\end{tabular}


PHASE I FOCUSED CMS/FS REPORT

L-AREA OIL AND CHEMICAL BASIN
WSRC-RP-96-106, REV. 1.1

FEBRUARY 1997

THIS PAGE INTENTIONALLY LEFT BLANK 


\section{EXECUTIVE SUMMARY}

This report presents the completed Resource Conservation and Recovery Act (RCRA) Comprehensive Environmental Response, Compensation, and Liability Act (CERCLA) Focused Corrective Measures Study/Feasibility Study (CMS/FS) for the L-Area Oil and Chemical Basin (LAOCB)/L-Area Acid Caustic Basin (LAACB) Solid Waste Management Unit/Operable Unit (SWMU/OU) at the Savannah River Site (SRS). The LAOCB and LAACB are located approximately $192 \mathrm{~m}$ (400 ft) southeast of L Reactor. Between 1961 and 1979, the LAOCB was operated as an unlined earthen basin for the purpose of disposing of small volumes of wastes that were not appropriate for discharge to local streams, regular seepage basins, or the waste management system. Liquid waste consisting of small volumes of radioactive oil and chemical wastewater were sent to the LAOCB from throughout the SRS, but came primarily from the reactor areas. The LAACB was operated from 1955 to 1968 as an unlined earthen basin for disposal of wastewater from the L-Area water treatment plant. The wastewater from this treatment plant consisted of dilute solutions of sulfuric acid and sodium hydroxide which had been used to regenerate ion exchange resins in the L-Area's power generation facility's water purification processes.

The LAOCB SWMU/OU includes the LAOCB sludge/soil, vegetation inside the LAOCB, soil,. concrete, and vegetation in the staging area on the north end of the LAOCB, soil adjacent to the LAOCB, the LAOCB pipelines and associated soil, the LAACB, and groundwater. The RCRA Facility Investigation/Remedial Investigation (RFI/RI) and the Baseline Risk Assessment (BRA) performed at this unit indicates that:

- LAOCB sludge/soil pose significant risk to human health

- LAOCB staging area on north end of basin poses significant risk to human health

- LAOCB pipelines pose potential future risk to human health

- soils adjacent to the LAOCB do not pose significant risk

- $\mathrm{LAACB}$ and associated pipeline do not pose significant risk 
- the extent of LAOCB groundwater contamination in the vicinity of the LAOCB has not been completely defined and requires further characterization

Results of the risk assessment conducted for the LAOCB identified potential significant carcinogenic risks associated with exposure to direct radiation and ingestion of soils/sediments at the LAOCB under hypothetical future land use scenarios. Significant risk related exposure pathways for the LAOCB soils include: (1) external radiation from basin soils, (2) ingestion of produce grown in basin soils, (3) inhalation of soil particulates, and (4) ingestion of basin soils. Two receptors are identified as having the potential for significant risk from both radiological and nonradiological (metals) contaminants: (1) the future on-unit resident and (2) the future onunit worker. Radionuclides and metals represent 100 percent of the risk above $1 \mathrm{E}-06$ for all applicable receptors, with greater than 99 percent of the total unit risk attributable to radionuclides that pose risk in excess of $1 \mathrm{E}-04$. The predominant risk drivers for these receptors are direct radiation from cobalt- 60 and cesium-137 and ingestion of soils from chromium and lead. Both the future on-unit resident and the future on-unit worker has significant risk from exposure to radionuclides and nonradionuclides (metals).

The BRA indicates that ingestion of soils from the LAOCB pipelines poses a potential hazard under future land use scenarios primarily from exposure to non-radioactive metals; however, it has been demonstrated that the metals that represent risk are naturally occurring in SRS soils. Results of the RFI/RI identified radioactive contamination in the interior of the LAOCB pipelines; however, specific radioisotopes were not identified and actual risk from this contamination was not calculated. Although the observed radioactive contamination on the internal surface does not currently represent risk to human health and the environment, future deterioration of the steel walls of the pipeline could potentially release contaminants to the environment and result in unacceptable risk. Man-made radioisotopes were not detected in pipeline soils and are not suspected to be migrating from the pipelines currently. No risk or hazards under the current and future land use scenarios were identified for soils adjacent to the LAOCB or the soils within and adjacent to the LAACB and associated pipeline. 
Since significant risks are associated with the waste remaining in the LAOCB, an evaluation of available remediation strategies was warranted. Because potential future risks are also associated with the LAOCB pipelines, an evaluation of remediation strategies applicable to the pipelines was also warranted. Therefore, a CMS/FS was conducted for the LAOCB sludge/soil and LAOCB pipelines. Since no significant risks are associated with the LAACB, evaluation of remediation strategies was not warranted and No Action is proposed. In addition, since the full extent of groundwater contamination has not been delineated, LAOCB groundwater remediation strategies will be evaluated following assessment of additional characterization efforts.

Remedial action objectives are based on the results of the BRA and on the evaluation of applicable or relevant and appropriate requirements (ARARs) and "to-be-considered" (TBC) information. The remedial action objectives were developed to guide the selection/evaluation of remedial technologies/alternatives. The remedial action objectives for the LAOCB sludge/soil are as follows:

1. reduce risks to human health and the environment associated with:

a. external exposure to radiological contaminants

b. inhalation of radiological contaminants

c. incidental ingestion of radiological contaminants in the surface soil at the unit

d. to prevent or mitigate the leaching of constituents of concern (COCs) to unit groundwater

2. meet concentration based remedial goal options (RGOs) for unit soils

The only remedial action objective associated with the LAOCB pipeline is the prevention/mitigation of the release of COCs to groundwater. 
The BRA indicated that LAOCB sludge/soil pose a potential threat to human health through exposure to sixteen primary COCs (>1E-04 risk) and five secondary COCs (1E-04 to 1E-06 risk). The BRA indicated that LAOCB pipeline soil pose a potential threat to human health through exposure to four primary COCs and two secondary COCs. These primary and secondary COCs are presented in Table ES-1.

RGOs were developed for the primary COCs (primarily radionuclides) which represent greater than 99 percent of the total unit risk (see Table ES-1). RGOs are human health risk-based calculations performed on COCs which are primary contributors of potential risk and/or adverse effects for the future resident scenario. In addition, linear regression analysis of gross alpha and gross beta activity in LAOCB sludge/soil indicate that gross alpha and grošs beta activity decreases to below background levels within a depth approximately $0.61 \mathrm{~m}(2 \mathrm{ft})$ from the top of the soils/sediments. Therefore, additional RGOs for the radionuclides in sludge/soil of the LAOCB are site specific background gross alpha and gross beta activities.

In general, RGOs for radionuclides in soil can only be achieved through off-unit removal/disposal alternatives. Therefore, no containment or treatment general response actions/alternatives will achieve RGOs. However, these alternatives meet the remedial action objective of preventing or mitigating the leaching of $\mathrm{COCs}$ to unit groundwater, and reduce risks to human health and the environment.

Development of remedial action objectives was not warranted for the soils adjacent to the LAOCB, within the LAACB, and adjacent to the LAACB and associated pipeline since no risk or hazards from the identified potential exposure pathways from either current or future land use scenarios exceed regulatory guidelines. In addition, no remedial action objectives were developed for groundwater in the vicinity of the Unit since the extent of groundwater contamination has not been fully delineated and further characterization is required. 
A full range of general response actions were developed in Section 2.0 for remediation of sludge/soil at the LAOCB and the LAOCB pipeline. General response actions for the LAOCB soils include no action, institutional controls, containment (e.g., physical barriers), in-situ treatment, ex-situ treatment, and disposal. General response actions for the LAOCB pipelines include no action, institutional controls, containment, in-situ treatment, and disposal. No action alternatives, as required by the National Oil and Hazardous Substance Contingency Plan (NCP), form the basis of comparison for more proactive corrective measures/remedial alternatives. Technologies were identified within each general response action and screened on the basis of effectiveness, implementability, and relative cost.

The NCP requires that a range of alternatives that include treatment to reduce toxicity, mobility, or volume of contaminants be developed and evaluated. The range of alternatives developed includes alternatives which immobilize residual chemicals or reduce the volume of contaminants or contaminated media and, to the maximum extent feasible, eliminate or minimize the need for long-term management. In addition, alternatives were developed which involve little or no treatment, but which provide protection to human health and the environment by preventing or controlling exposure to the contaminants through engineering and institutional controls. Potential alternatives were developed from the lists of retained technologies to address LAOCB sludge/soil (Table ES-2) and LAOCB pipelines (Table ES-3). The remedial alternatives. developed for the LAOCB soils are:

S-1 No Action

S-2 Backfill and Cap

S-3 Slurry Cut-Off Wall \& Cap

S-4 In Situ Stabilization \& Cap

S-5 In-Situ Vitrification \& Cap

S-6 Ex Situ Stabilization \& Cap

S-7 Ex Situ Vitrification \& Cap

S-8 Excavate and Disposal at SRS Soil Consolidation Facility

S-9 Excavate and Disposal at Nevada Test Site

S-10 Excavate and Disposal at Envirocare Facility 
The remedial alternatives developed for the LAOCB pipelines are:

P-1 No Action

P-2 Institutional/Administrative Controls

P-3 Capping

P-4 In Situ Solidification/Stabilization (S/S)

P-5 In Situ High Pressure Wash and In Situ S/S

P-6 In Situ S/S, Excavation, and Disposal in the LAOCB

P-7 In Situ S/S, Excavation, and Disposal at Nevada Test Site

Comprehensive alternatives presented above were screened on the basis of effectiveness, implementability, and cost (see Tables ES-2 and ES-3). The costs for alternatives were developed only for comparison purposes and are not intended to forecast a budgetary estimate of actual expenditures. Soil/sediment alternatives involving vitrification were rejected on the basis of cost. Disposal of LAOCB sludge/soil and the LAOCB pipelines at the conceptual SRS Soils/Debris Consolidation Facility (SDCF) was temporarily rejected because the facility has not been sited, designed, or constructed and no waste acceptance criteria have been established; however, should the SDCF become operational in the future, disposal of LAOCB wastes at the facility could be re-evaluated. Disposal of soils/sediment and LAOCB pipelines at the Envirocare Facility was rejected on the basis of costs in relation to disposal at the Nevada Test Site (NTS) Facility. Alternative P-2 (institutional controls), and Alternatives P-4 and P-5 (in-situ $\mathrm{S} / \mathrm{S}$ ) for the LAOCB pipeline were rejected on the basis of not being protective of human health and the environment.

Radionuclides are unique contaminants with a very limited selection of remedial responses/technologies. Six alternatives for LAOCB soils and four alternatives for the LAOCB pipelines retained for detailed analysis are as follows:

\section{$\underline{\text { LAOCB Soil Alternatives }}$}

S-1 No Action

S-2 Backfill and Cap

S-3 Slurry Cut-Off Wall \& Cap

S-4 In Situ Stabilization \& Cap

S-5 Ex Situ Stabilization \& Cap

S-6 Excavate and Disposal at Nevada Test Site

\section{LAOCB Pipeline Alternatives}

P-1 No Action

P-2 Capping

P-3 In Situ S/S, Excavation, and Disposal in the LAOCB

P-4 In Situ S/S, Excavation, and

Disposal at Nevada Test Site 
Tables ES-4 and ES-5 provide summaries of the comparative analysis of alternatives relative to the NCP criteria for the LAOCB and LAOCB pipelines, respectively. These technologies, or any combination of them, are equally if not more applicable to the treatment of metals as they are for radionuclides. Since radionuclides and metals represent 100 percent of total unit risk above $1 \mathrm{E}$ 06, and radionuclides and metals have very similar physical and chemical properties, these remedial alternatives will be effective for treating all contaminants with risk values above 1E-06. 
THIS PAGE INTENTIONALLY LEFT BLANK 
EXECUTIVE SUMMARY

TABLES 
PHASE I FOCUSED CMS/FS REPORT

WSRC-RP-96-106, REV. 1.1

L-AREA OIL AND CHEMICAL BASIN

FEBRUARY 1997

THIS PAGE INTENTIONALLY LEFT BLANK 
Table ES-1

Primary and Secondary COCs and RGOs

\begin{tabular}{|c|c|c|}
\hline $\begin{array}{l}\text { Contaminants of } \\
\text { Concern (COCs) }\end{array}$ & Units & $\begin{array}{l}\text { RGOs to Achieve } \\
\text { 1E-06 Risk } \\
\text { and HI of } 1 \\
\text { Future Resident } \\
\text { Adult and Child }\end{array}$ \\
\hline \multicolumn{3}{|c|}{ 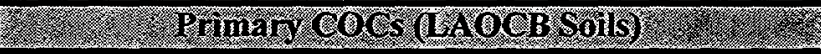 } \\
\hline $\begin{array}{l}\text { Americium-241 } \\
\text { Antimony-125 } \\
\text { Cesium-137 } \\
\text { Cobalt-60 } \\
\text { Curium-244 } \\
\text { Europium-152 } \\
\text { Europium-154 } \\
\text { Plutonium-238 } \\
\text { Plutonium-239 } \\
\text { Potassium-40 } \\
\text { Strontium-90 } \\
\text { Uranium-238+D } \\
\text { Uranium-234 } \\
\text { Uranium-235+D } \\
\text { Aluminum } \\
\text { Cadmium } \\
\text { Chromium } \\
\text { Lead } \\
\text { Nickel } \\
\text { Vanadium }\end{array}$ & $\begin{array}{l}\mathrm{pCi} / \mathrm{g} \\
\mathrm{pCi} / \mathrm{g} \\
\mathrm{pCi} / \mathrm{g} \\
\mathrm{pCi} / \mathrm{g} \\
\mathrm{pCi} / \mathrm{g} \\
\mathrm{pCi} / \mathrm{g} \\
\mathrm{pCi} / \mathrm{g} \\
\mathrm{pCi} / \mathrm{g} \\
\mathrm{pCi} / \mathrm{g} \\
\mathrm{pCi} / \mathrm{g} \\
\mathrm{pCi} / \mathrm{g} \\
\mathrm{pCi} / \mathrm{g} \\
\mathrm{pCi} / \mathrm{g} \\
\mathrm{pCi} / \mathrm{g} \\
\mathrm{mg} / \mathrm{kg} \\
\mathrm{mg} / \mathrm{kg} \\
\mathrm{mg} / \mathrm{kg} \\
\mathrm{mg} / \mathrm{kg} \\
\mathrm{mg} / \mathrm{kg} \\
\mathrm{mg} / \mathrm{kg}\end{array}$ & $\begin{array}{l}2.60 \mathrm{E}+00 \\
6.30 \mathrm{E}-02 \\
3.70 \mathrm{E}-02 \\
8.80 \mathrm{E}-03 \\
4.90 \mathrm{E}+00 \\
2.10 \mathrm{E}-02 \\
1.90 \mathrm{E}-02 \\
3.60 \mathrm{E}+00 \\
3.40 \mathrm{E}+00 \\
1.20 \mathrm{E}-01 \\
3.90 \mathrm{E}-01 \\
1.40 \mathrm{E}+00 \\
2.50 \mathrm{E}+01 \\
3.10 \mathrm{E}-01 \\
7.80 \mathrm{E}+04 \\
1.20 \mathrm{E}+01 \\
3.40 \mathrm{E}+02 \\
2.80 \mathrm{E}+01 \\
4.70 \mathrm{E}+02 \\
5.40 \mathrm{E}+01\end{array}$ \\
\hline \multicolumn{3}{|c|}{ 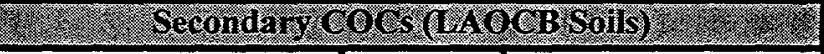 } \\
\hline $\begin{array}{l}\text { Actinium-228 } \\
\text { Thallium-208 } \\
\text { Bismuth-214 } \\
\text { Potassium-40 } \\
\text { Beryllium }\end{array}$ & & $\begin{array}{l}\text { ND } \\
\text { ND } \\
\text { ND } \\
\text { ND } \\
\text { ND }\end{array}$ \\
\hline \multicolumn{3}{|c|}{ 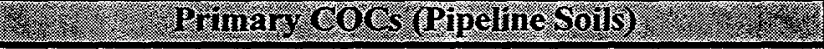 } \\
\hline $\begin{array}{l}\text { Aluminum } \\
\text { Arsenic } \\
\text { Lead } \\
\text { Thallium }\end{array}$ & $\begin{array}{l}\mathrm{mg} / \mathrm{kg} \\
\mathrm{mg} / \mathrm{kg} \\
\mathrm{mg} / \mathrm{kg} \\
\mathrm{mg} / \mathrm{kg}\end{array}$ & $\begin{array}{l}7.80 \mathrm{E}+04 \\
1.90 \mathrm{E}+01 \\
2.80 \mathrm{E}+01 \\
6.10 \mathrm{E}+00\end{array}$ \\
\hline 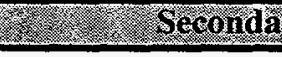 & & nons \\
\hline $\begin{array}{l}\text { Beryllium } \\
\text { Thallium-208 }\end{array}$ & & $\begin{array}{l}\text { ND } \\
\text { ND }\end{array}$ \\
\hline
\end{tabular}

ND - RGOs were not determined 
TABLE ES-2

SUMMARY OF REMEDIAL ALTERNATIVES SCREENING

L-AREA OIL AND CHEMICAL BASIN SOIL

SAVANNAH RIVER SITE

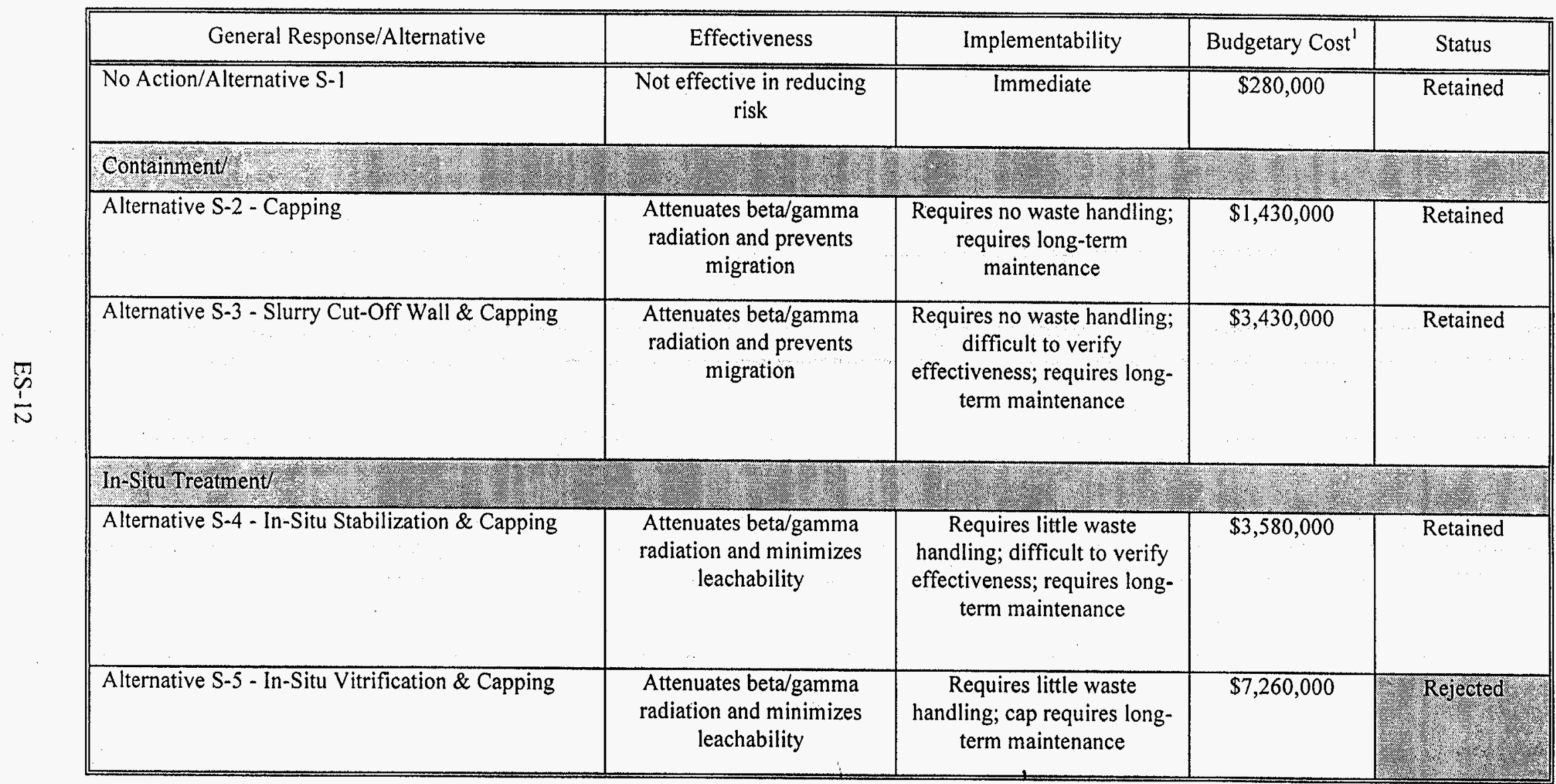


TABLE ES-2 (CONTINUED)

SUMMARY OF REMEDIAL ALTERNATIVES SCREENING L-AREA OIL AND CHEMICAL BASIN SOIL

SAVANNAH RIVER SITE

\begin{tabular}{|c|c|c|c|c|}
\hline General Response/Alternative & Effectiveness & Implementability & Budgetary Cost ${ }^{1}$ & Status \\
\hline \multicolumn{5}{|l|}{ Ex-Situ Treatment } \\
\hline Alternative S-6 - Ex-Situ Stabilization \& Capping & $\begin{array}{c}\text { Attenuates beta/gamma } \\
\text { radiation and minimizes } \\
\text { leachability }\end{array}$ & $\begin{array}{l}\text { Requires pre-excavation } \\
\text { treatment for waste handling } \\
\text { purposes; extensive waste } \\
\text { handling and long-term cap } \\
\text { maintenance }\end{array}$ & $\$ 4,370,000$ & Retained \\
\hline \multicolumn{5}{|l|}{ Orf-Unit Disposall } \\
\hline $\begin{array}{l}\text { Alternative S-8 - Excavation \& Disposal at the Soils } \\
\text { Consolidation Facility }\end{array}$ & $\begin{array}{l}\text { Removes source of } \\
\text { contamination; little } \\
\text { residual risk to human } \\
\text { health or the environment }\end{array}$ & $\begin{array}{l}\text { Requires pre- \& post- } \\
\text { excavation treatment for } \\
\text { waste handling/packaging } \\
\text { purposes; extensive waste } \\
\text { handling and transport; } \\
\text { increased worker exposure; } \\
\text { significant risk to public }\end{array}$ & Unknown $^{2}$ & $\operatorname{emp}$ \\
\hline $\begin{array}{l}\text { Alternative S-9 - Excavation \& Disposal at the } \\
\text { Nevada Test Site Facility }\end{array}$ & $\begin{array}{l}\text { Removes source of } \\
\text { contamination; little } \\
\text { residual risk to human } \\
\text { health or the environment }\end{array}$ & $\begin{array}{l}\text { Requires pre- \& post- } \\
\text { excavation treatment for } \\
\text { waste handling/packaging } \\
\text { purposes; extensive waste } \\
\text { handling and transport; } \\
\text { increased wórker exposure; } \\
\text { significant risk to public }\end{array}$ & $\$ 9,100,000$ & Retained \\
\hline
\end{tabular}


TABLE ES-2 (CONTINUED)

SUMMARY OF REMEDIAL ALTERNATIVES SCREENING

L-AREA OIL AND CHEMICAL BASIN SOIL

SAVANNAH RIVER SITE

\begin{tabular}{|l|c|c|c|}
\hline \multicolumn{1}{|c|}{ General Response/Alternative } & Effectiveness & Implementability $^{\text {Alternative S-10 - Excavation \& Disposal at the }}$ \\
Envirocare Facility & $\begin{array}{c}\text { Removes source of } \\
\text { contamination; little } \\
\text { residual risk to human } \\
\text { health or the environment }\end{array}$ & $\begin{array}{c}\text { Bequires pre- \& post- } \\
\text { excavation treatment for } \\
\text { waste handling/packaging } \\
\text { purposes; extensive waste } \\
\text { handling and transport; } \\
\text { increased worker exposure; } \\
\text { significant risk to public }\end{array}$ \\
\hline
\end{tabular}

${ }^{1}$ Costs are developed for comparison purposes only and are not intended to forecast actual expenditures.

${ }^{2}$ Costs are not known since the Soils Consolidation Facility has not been sited, designed, or constructed.

$\mathrm{S} / \mathrm{S}=$ Stabilization/Solidification 
TABLE ES-3

SUMMARY OF REMEDIAL ALTERNATIVES SCREENING

L-AREA OIL AND CHEMICAL BASIN PIPELINES

SAVANNAH RIVER SITE

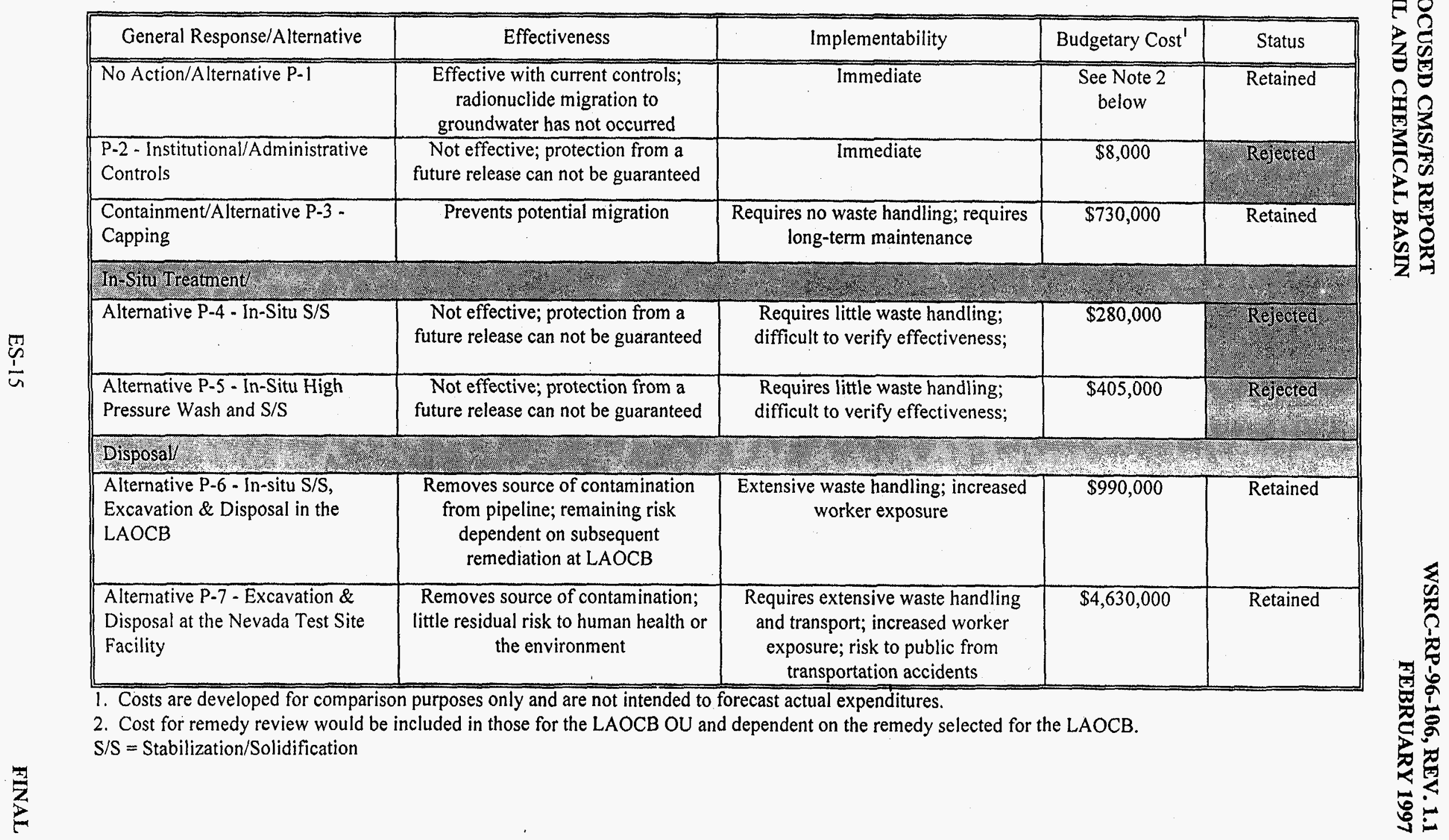


TABLE ES-4

COMPARATIVE ANALYSIS OF SOIL/SEDIMENT ALTERNATIVES

L-AREA OIL AND CHEMICAL BASIN

SAVANNAH RIVER SITE

\begin{tabular}{|c|c|c|c|c|c|c|}
\hline \multirow{2}{*}{ Criterion } & \multicolumn{6}{|c|}{ LAOCB Soil Remedial Alternatives } \\
\hline & $\begin{array}{l}\text { Alternative S-1 } \\
\text { No Action }\end{array}$ & $\begin{array}{l}\text { Alternative } S-2 \\
\text { Capping }\end{array}$ & $\begin{array}{l}\text { Alternative S-3 } \\
\text { Slurry Cut-Off Wall } \\
\text { \& Capping }\end{array}$ & $\begin{array}{l}\text { Alternative S-4 } \\
\text { In-Situ S/S \& } \\
\text { Capping }\end{array}$ & $\begin{array}{l}\text { Alternative S-5 } \\
\text { Ex-Situ S/S \& } \\
\text { Capping }\end{array}$ & $\begin{array}{c}\text { Alternative S-6 } \\
\text { Disposal at the Nevada } \\
\text { Test Site }\end{array}$ \\
\hline Human Health & $\begin{array}{l}\text { Protective as long as } \\
\text { institutional controls } \\
\text { are maintained }\end{array}$ & Protective & Protective & Protective & Protective & Protective \\
\hline Environment & $\begin{array}{l}\text { Protective as long as } \\
\text { clay layer beneath } \\
\text { Basin prevents } \\
\text { migration }\end{array}$ & Protective & Protective & Protective & Protective & Protective \\
\hline Chemical-specific & $\begin{array}{c}\text { Meets } \\
\text { TSCA/UMTRCA } \\
\text { levels; would not } \\
\text { meet } 40 \text { CFR } 191 \text { or } \\
\text { DOE Order } 5400.5 \\
\text { (TBC) under } \\
\text { hypothetical future } \\
\text { conditions }\end{array}$ & $\begin{array}{c}\text { Meets } \\
\text { TSCA/UMTRCA } \\
\text { levels; Complies } \\
\text { with 40 CFR 191 } \\
\text { and DOE Order } \\
5400.5 \text { (TBC) }\end{array}$ & $\begin{array}{c}\text { Meets } \\
\text { TSCA/UMTRCA } \\
\text { levels; Complies } \\
\text { with 40 CFR 191 } \\
\text { and DOE Order } \\
5400.5 \text { (TBC) }\end{array}$ & $\begin{array}{c}\text { Meets } \\
\text { TSCA/UMTRCA } \\
\text { levels; Complies } \\
\text { with } 40 \text { CFR 191 } \\
\text { and DOE Order } \\
5400.5 \text { (TBC) } \\
\text { I }\end{array}$ & $\begin{array}{c}\text { Meets } \\
\text { TSCA/UMTRCA } \\
\text { levels; Complies } \\
\text { with 40 CFR 191 } \\
\text { and DOE Order } \\
5400.5(\mathrm{TBC})\end{array}$ & $\begin{array}{c}\text { Meets } \\
\text { TSCA/UMTRCA } \\
\text { levels; Complies with } \\
\text { 40 CFR } 191 \text { and DOE } \\
\text { Order 5400.5 (TBC) }\end{array}$ \\
\hline Location-specific & Not Applicable & None & None & None & None & None \\
\hline
\end{tabular}


TABLE ES-4 (CONTINUED)

COMPARATIVE ANALYSIS OF SOIL/SEDIMENT ALTERNATIVES

L-AREA OIL AND CHEMICAL BASIN

SAVANNAH RIVER SITE

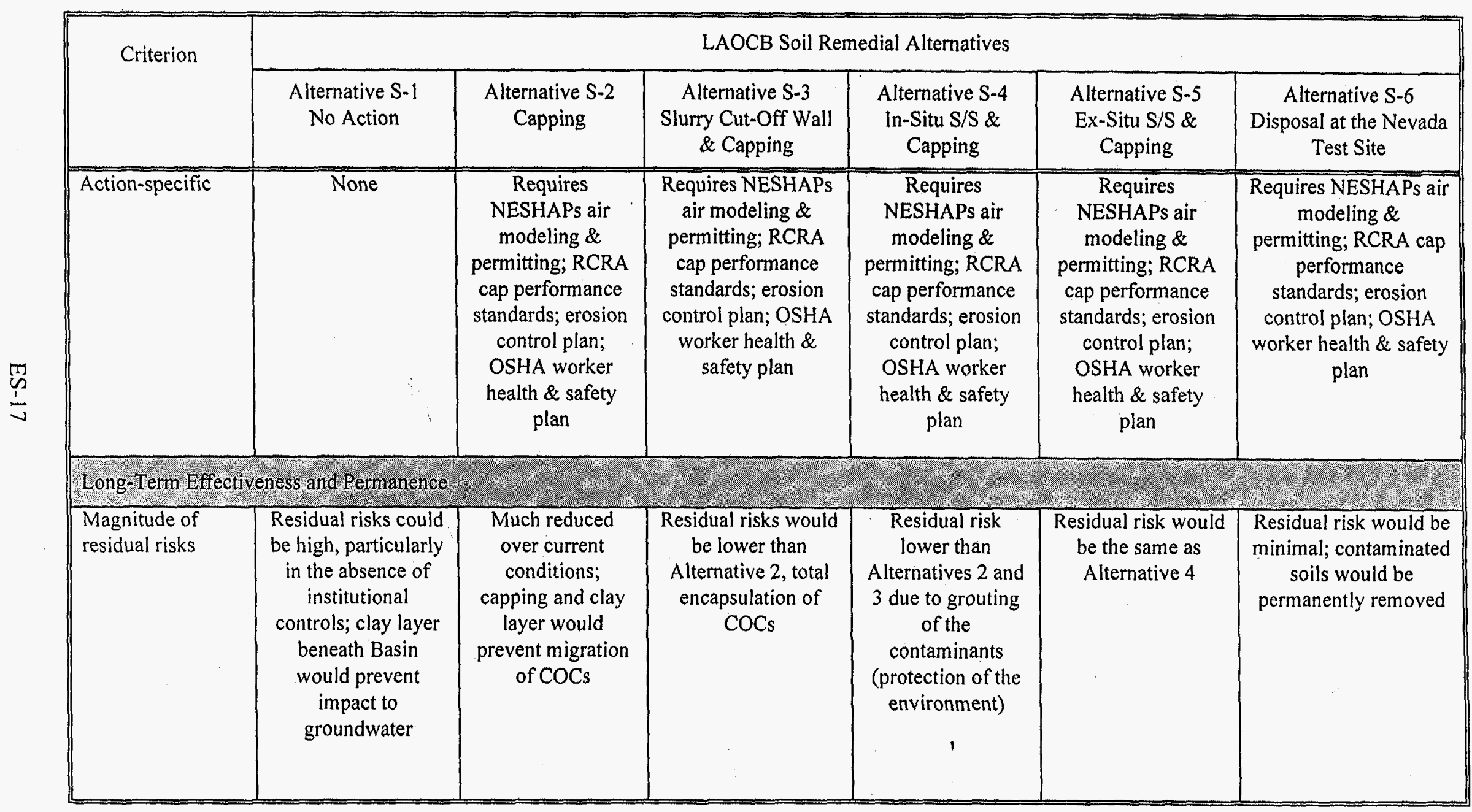


TABLE ES-4 (CONTINUED)

COMPARATIVE ANALYSIS OF SOIL/SEDIMENT ALTERNATIVES

L-AREA OIL AND CHEMICAL BASIN

SAVANNAH RIVER SITE

\begin{tabular}{|c|c|c|c|c|c|c|}
\hline \multirow{2}{*}{ Criterion } & \multicolumn{6}{|c|}{ LAOCB Soil Remedial Alternatives } \\
\hline & $\begin{array}{c}\text { Alternative S-1 } \\
\text { No Action }\end{array}$ & $\begin{array}{c}\text { Alternative S-2 } \\
\text { Capping }\end{array}$ & $\begin{array}{l}\text { Alternative S-3 } \\
\text { Slurry Cut-Off Wall } \\
\text { \& Capping }\end{array}$ & $\begin{array}{l}\text { Alternative S-4 } \\
\text { In-Situ S/S \& } \\
\text { Capping }\end{array}$ & $\begin{array}{l}\text { Alternative S-5 } \\
\text { Ex-Situ S/S \& } \\
\text { Capping }\end{array}$ & $\begin{array}{c}\text { Alternative S-6 } \\
\text { Disposal at the Nevada } \\
\text { Test Site }\end{array}$ \\
\hline $\begin{array}{l}\text { Adequacy of } \\
\text { controls }\end{array}$ & $\begin{array}{l}\text { Existing } \\
\text { institutional controls } \\
\text { are effective for the } \\
\text { protection of human } \\
\text { health, but cannot } \\
\text { be guaranteed; } \\
\text { adequacy of the clay } \\
\text { layer has proven } \\
\text { effective, but would } \\
\text { not be verified }\end{array}$ & $\begin{array}{l}\text { Existing and } \\
\text { supplemental } \\
\text { institutional } \\
\text { controls would be } \\
\text { effective; cap and } \\
\text { the clay layer } \\
\text { beneath the Basin } \\
\text { would prevent } \\
\text { migration of } \\
\text { COCs }\end{array}$ & $\begin{array}{c}\text { Existing and } \\
\text { supplemental } \\
\text { institutional controls } \\
\text { would be effective; } \\
\text { slurry wall, cap and } \\
\text { the clay layer } \\
\text { beneath the Basin } \\
\text { would prevent } \\
\text { migration of COCs }\end{array}$ & $\begin{array}{l}\text { Existing and } \\
\text { supplemental } \\
\text { institutional } \\
\text { controls would be } \\
\text { effective and } \\
\text { grouting of the } \\
\text { contaminated soils } \\
\text { would further } \\
\text { limit risk to the } \\
\text { environment }\end{array}$ & $\begin{array}{l}\text { Existing and } \\
\text { supplemental } \\
\text { institutional } \\
\text { controls would be } \\
\text { effective and } \\
\text { grouting of the } \\
\text { contaminated soils } \\
\text { would further limit } \\
\text { risk to the } \\
\text { environment }\end{array}$ & $\begin{array}{l}\text { No controls required; } \\
\text { could be released for } \\
\text { unrestricted land use }\end{array}$ \\
\hline Treatment type & No active treatment & $\begin{array}{l}\text { No active } \\
\text { treatment }\end{array}$ & No active treatment & $\begin{array}{c}\text { Stabilization/ } \\
\text { solidification of } \\
\text { the contaminated } \\
\text { soil }\end{array}$ & $\begin{array}{l}\text { Stabilization/ } \\
\text { solidification of the } \\
\text { contaminated soil }\end{array}$ & None \\
\hline
\end{tabular}


TABLE ES-4 (CONTINUED)

COMPARATIVE ANALYSIS OF SOIL/SEDIMENT ALTERNATIVES

L-AREA OIL AND CHEMICAL BASIN

SAVANNAH RIVER SITE

\begin{tabular}{|c|c|c|c|c|c|c|}
\hline \multirow{2}{*}{ Criterion } & \multicolumn{6}{|c|}{ LAOCB Soil Remedial Alternatives } \\
\hline & $\begin{array}{l}\text { Alternative S-1 } \\
\text { No Action }\end{array}$ & $\begin{array}{l}\text { Alternative S-2 } \\
\text { Capping }\end{array}$ & $\begin{array}{l}\text { Alternative S-3 } \\
\text { Slurry Cut-Off Wall } \\
\text { \& Capping }\end{array}$ & $\begin{array}{l}\text { Alternative S-4 } \\
\text { In-Situ S/S \& } \\
\text { Capping }\end{array}$ & $\begin{array}{l}\text { Alternative S-5 } \\
\text { Ex-Situ S/S \& } \\
\text { Capping }\end{array}$ & $\begin{array}{c}\text { Alternative S-6 } \\
\text { Disposal at the Nevada } \\
\text { Test Site }\end{array}$ \\
\hline $\begin{array}{l}\text { Reduction of } \\
\text { toxicity, mobility } \\
\text { or volume }\end{array}$ & $\begin{array}{l}\text { None through } \\
\text { treatment }\end{array}$ & $\begin{array}{l}\text { Capping and the } \\
\text { clay layer beneath } \\
\text { the Basin would } \\
\text { effectively reduce } \\
\text { contaminant } \\
\text { mobility as long as } \\
\text { cap integrity is } \\
\text { maintained; not a } \\
\text { permanent } \\
\text { reduction in } \\
\text { contaminant } \\
\text { mobility }\end{array}$ & $\begin{array}{l}\text { Slurry wall, capping } \\
\text { and the clay layer } \\
\text { beneath the Basin } \\
\text { would effectively } \\
\text { reduce contaminant } \\
\text { mobility as long as } \\
\text { cap integrity is } \\
\text { maintained; not a } \\
\text { permanent reduction } \\
\text { in contaminant } \\
\text { mobility }\end{array}$ & $\begin{array}{l}\text { Permanently } \\
\text { reduce } \\
\text { contaminant } \\
\text { mobility in the } \\
\text { soils }\end{array}$ & $\begin{array}{l}\text { Permanently reduce } \\
\text { contaminant } \\
\text { mobility in the soils }\end{array}$ & $\begin{array}{l}\text { Contaminated soils } \\
\text { removed, but relocated }\end{array}$ \\
\hline \multicolumn{7}{|c|}{ Short-Term Effectiveness } \\
\hline $\begin{array}{l}\text { Risk to remedial } \\
\text { workers }\end{array}$ & $\begin{array}{c}\text { None; would } \\
\text { involve no handling } \\
\text { of contaminated } \\
\text { media }\end{array}$ & Minimal & Minimal & Low & $\begin{array}{c}\text { Medium; volume of } \\
\text { soils excavated: } \\
760 \mathrm{~m}^{3}\left(27,000 \mathrm{ft}^{3}\right. \\
\left.1,000 \mathrm{yd}^{3}\right)\end{array}$ & $\begin{array}{l}\text { Medium to high; } \\
\text { volume of soils } \\
\text { excavated and } \\
\text { transported: } 760 \mathrm{~m}^{3} \\
\left(27,000 \mathrm{ft}^{3}, 1,000 \mathrm{yd}^{3}\right)\end{array}$ \\
\hline $\begin{array}{l}\text { Risk to } \\
\text { community }\end{array}$ & Negligible & Minimal & Minimal & Minimal & Minimal & $\begin{array}{l}\text { Medium; would } \\
\text { involve transport of } \\
\text { soils to the NTS }\end{array}$ \\
\hline $\begin{array}{l}\text { Construction } \\
\text { schedule }\end{array}$ & $\begin{array}{c}\text { Immediately } \\
\text { implementable }\end{array}$ & 3 months & 6 months & 12 months & 15 months & 3 months \\
\hline
\end{tabular}


TABLE ES-4 (CONTINUED)

COMPARATIVE ANALYSIS OF SOIL/SEDIMENT ALTERNATIVES

L-AREA OIL AND CHEMICAL BASIN

SAVANNAH RIVER SITE

\begin{tabular}{|c|c|c|c|c|c|c|}
\hline \multirow{2}{*}{ Criterion } & \multicolumn{6}{|c|}{ LAOCB Soil Remedial Alternatives } \\
\hline & $\begin{array}{l}\text { Alternative S-1 } \\
\text { No Action }\end{array}$ & $\begin{array}{l}\text { Alternative S-2 } \\
\text { Capping }\end{array}$ & $\begin{array}{l}\text { Alternative S-3 } \\
\text { Slurry Cut-Off Wall } \\
\text { \& Capping }\end{array}$ & $\begin{array}{l}\text { Alternative S-4 } \\
\text { In-Situ S/S \& } \\
\text { Capping }\end{array}$ & $\begin{array}{c}\text { Alternative S-5 } \\
\text { Ex-Situ S/S \& } \\
\text { Capping }\end{array}$ & $\begin{array}{c}\text { Alternative S-6 } \\
\text { Disposal at the Nevada } \\
\text { Test Site }\end{array}$ \\
\hline Potential concerns & $\begin{array}{l}\text { Potential for public } \\
\text { concern if no action } \\
\text { is implemented }\end{array}$ & $\begin{array}{l}\text { Potential for } \\
\text { public concern } \\
\text { since no treatment } \\
\text { is performed }\end{array}$ & $\begin{array}{l}\text { Potential for public } \\
\text { concern since no } \\
\text { treatment is } \\
\text { performed }\end{array}$ & None & $\begin{array}{l}\text { Medium; would } \\
\text { require pre- } \\
\text { excavation } \\
\text { treatment for waste } \\
\text { handling purposes }\end{array}$ & $\begin{array}{l}\text { High; would involve } \\
\text { transport of soils } \\
\text { outside SRS } \\
\text { boundaries; would } \\
\text { require pre- \& post- } \\
\text { excavation treatment } \\
\text { for waste handling \& } \\
\text { packaging purposes }\end{array}$ \\
\hline $\begin{array}{l}\text { Relative } \\
\text { implementability }\end{array}$ & $\begin{array}{c}\text { Readily } \\
\text { implementable }\end{array}$ & $\begin{array}{l}\text { Readily } \\
\text { implementable, } \\
\text { but would require } \\
\text { much more effort } \\
\text { than No Action }\end{array}$ & $\begin{array}{c}\text { Readily } \\
\text { implementable; } \\
\text { would require more } \\
\text { effort than capping } \\
\text { alone (Alt 2) }\end{array}$ & $\begin{array}{l}\text { Readily } \\
\text { implementable; } \\
\text { would require } \\
\text { more effort than } \\
\text { capping alone } \\
\text { (Alt 2) }\end{array}$ & $\begin{array}{l}\text { Implementable; } \\
\text { however, waste } \\
\text { handling may cause } \\
\text { down time during } \\
\text { remediation; also } \\
\text { requires pre- } \\
\text { excavation } \\
\text { treatment }\end{array}$ & $\begin{array}{l}\text { Implementable; } \\
\text { however, waste } \\
\text { handling may cause } \\
\text { down time during } \\
\text { remediation; also } \\
\text { requires pre- \& post- } \\
\text { excavation treatment }\end{array}$ \\
\hline
\end{tabular}


TABLE ES-4 (CONTINUED)

COMPARATIVE ANALYSIS OF SOIL/SEDIMENT ALTERNATIVES

L-AREA OIL AND CHEMICAL BASIN

SAVANNAH RIVER SITE

\begin{tabular}{|c|c|c|c|c|c|c|}
\hline Criterion & $\begin{array}{l}\text { Alternative S-1 } \\
\text { No Action }\end{array}$ & $\begin{array}{l}\text { Alternative S-2 } \\
\text { Capping }\end{array}$ & $\begin{array}{l}\text { Alternative S-3 } \\
\text { Slurry Cut-Off Wall } \\
\quad \& \text { Capping }\end{array}$ & $\begin{array}{l}\text { Alternative S-4 } \\
\text { In-Situ S/S \& } \\
\text { Capping }\end{array}$ & $\begin{array}{l}\text { Alternative S-5 } \\
\text { Ex-Situ S/S \& } \\
\text { Capping }\end{array}$ & $\begin{array}{c}\text { Alternative S- } 6 \\
\text { Disposal at the Nevada } \\
\text { Test Site }\end{array}$ \\
\hline st* & & & & & (2) & 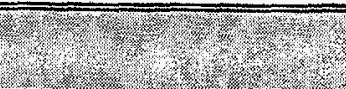 \\
\hline $\begin{array}{l}\text { Basis for O\&M } \\
\text { costs }\end{array}$ & 30 years & 30 years & 30 years & 30 years & 30 years & Not applicable \\
\hline $\begin{array}{l}\text { Present worth } \\
\text { capital costs }\end{array}$ & $\$ 0$ & $\$ 1,000,000$ & $\$ 3,000,000$ & $\$ 3,150,000$ & $\$ 3,940,000$ & $\$ 9,100,000$ \\
\hline $\begin{array}{l}\text { Present worth } \\
\text { O\&M costs }\end{array}$ & $\$ 280,000$ & $\$ 430,000$ & $\$ 430,000$ & $\$ 430,000$ & $\$ 430,000$ & $\$ 0$ \\
\hline $\begin{array}{l}\text { Total present } \\
\text { worth costs }\end{array}$ & $\$ 280,000$ & $\$ 1,430,000$ & $\$ 3,430,000$ & $\$ 3,580,000$ & $\$ 4,370,000$ & $\$ 9,100,000$ \\
\hline
\end{tabular}

${ }^{*}$ Costs are developed for comparison purposes only and are not intended to forecast actual expenditures.

$\mathrm{S} / \mathrm{S}=$ Stabilization/Solidification 
TABLE ES-5

COMPARATIVE ANALYSIS OF PIPELINE ALTERNATIVES

L-AREA OIL AND CHEMICAL BASIN

SAVANNAH RIVER SITE

\begin{tabular}{|c|c|c|c|c|}
\hline \multirow[t]{2}{*}{ Criterion } & \multicolumn{4}{|c|}{ LAOCB Pipelines Remedial Alternatives } \\
\hline & Alternative P-1 No Action & Alternative P-2 Capping & $\begin{array}{c}\text { Alternative } \mathrm{P}-3 \text { In-situ } \mathrm{S} / \mathrm{S} \\
\text { Excavation \& Disposal in the } \\
\text { LAOCB }\end{array}$ & $\begin{array}{l}\text { Alternative P-4 In-situ S/S } \\
\text { Excavation and Disposal at } \\
\text { the Nevada Test Site }\end{array}$ \\
\hline \multicolumn{5}{|l|}{ Overall Protectiveness } \\
\hline Human Health & Not Protective & Protective & Protective & Protective \\
\hline Environment & Not Protective & Protective & Protective & Protective \\
\hline Chemical-specific & $\begin{array}{c}\text { Compliance with DOE Order } \\
5400.5 \text { cannot be determined } \\
\text { until further investigation is } \\
\text { conducted }^{1}\end{array}$ & $\begin{array}{l}\text { Would comply with DOE } \\
\text { Order } 5400.5\end{array}$ & $\begin{array}{l}\text { Would comply with DOE } \\
\text { Order } 5400.5\end{array}$ & $\begin{array}{l}\text { Would comply with DOE } \\
\text { Order } 5400.5\end{array}$ \\
\hline Location-specific & None & None & None & None \\
\hline \multicolumn{5}{|c|}{ Long-Term Effectiveness and Performance } \\
\hline Magnitude of residual risks & $\begin{array}{l}\text { Risk would include intrusion } \\
\text { into the pipeline, and future } \\
\text { release of contaminants due } \\
\text { to degradation of the } \\
\text { pipelines integrity }\end{array}$ & $\begin{array}{l}\text { Residual risks would be } \\
\text { lower than Alternative 1; } \\
\text { capping would decreases } \\
\text { access, migration and } \\
\text { external exposure potential }\end{array}$ & $\begin{array}{l}\text { Residual risk would be } \\
\text { minimal; the pipeline would } \\
\text { be grouted in place and } \\
\text { permanently removed; } \\
\text { contaminants would be } \\
\text { transferred to the basin to be } \\
\text { stabilized further }\end{array}$ & $\begin{array}{l}\text { Residual risk would be } \\
\text { minimal; the pipeline would } \\
\text { be permanently removed }\end{array}$ \\
\hline
\end{tabular}


TABLE ES-5 (CONTINUED)

COMPARATIVE ANALYSIS OF PIPELINE ALTERNATIVES

L-AREA OIL AND CHEMICAL BASIN

SAVANNAH RIVER SITE

\begin{tabular}{|c|c|c|c|c|}
\hline Criterion & \multicolumn{4}{|c|}{ LAOCB Pipelines Remedial Alternatives } \\
\hline Adequacy of controls & $\begin{array}{l}\text { Existing pipeline appears to } \\
\text { prevent migration of } \\
\text { radionuclides, but migration } \\
\text { or protection against human } \\
\text { intrusion cannot be } \\
\text { guaranteed }\end{array}$ & $\begin{array}{l}\text { Capping would further } \\
\text { prevent migration of } \\
\text { contaminants by limiting } \\
\text { infiltration }\end{array}$ & $\begin{array}{l}\text { No controls required; could } \\
\text { be released for unrestricted } \\
\text { land use }\end{array}$ & $\begin{array}{l}\text { No controls required; could } \\
\text { be released for unrestricted } \\
\text { land use }\end{array}$ \\
\hline \multicolumn{5}{|c|}{ Reduction of Toxicity, Mobility or Volume: } \\
\hline Treatment type & No active treatment & No active treatment & In-Situ S/S & In-Situ S/S \\
\hline $\begin{array}{l}\text { Reduction of toxicity, } \\
\text { mobility or volume }\end{array}$ & None through treatment & $\begin{array}{l}\text { None through treatment, but } \\
\text { capping would decrease the } \\
\text { mobility of contaminants }\end{array}$ & $\begin{array}{l}\text { Grouting the pipeline would } \\
\text { reduce mobility }\end{array}$ & $\begin{array}{l}\text { Grouting the pipeline would } \\
\text { reduce mobility }\end{array}$ \\
\hline Risk to community & Negligible & Negligible & Minimal & $\begin{array}{l}\text { Medium; would involve } \\
\text { transport of contaminated } \\
\text { media to the NTS }\end{array}$ \\
\hline Construction schedule & Immediately implementable & 2 months & 2 months & 3 months \\
\hline
\end{tabular}


TABLE ES-5 (CONTINUED)

COMPARATIVE ANALYSIS OF PIPELINE ALTERNATIVES

L-AREA OIL AND CHEMICAL BASIN

SAVANNAH RIVER SITE

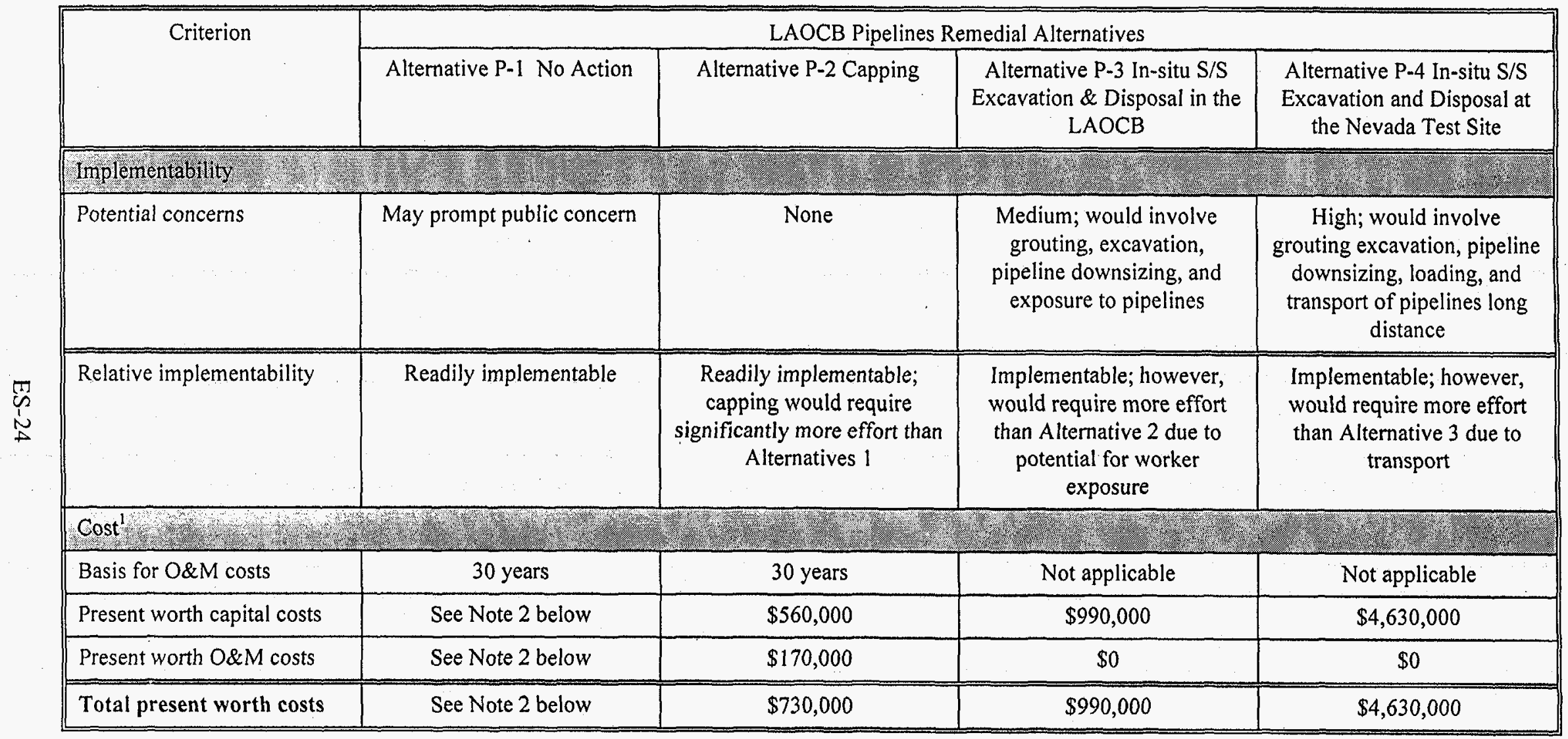

1. Costs are developed for comparison purposes only and are not intended to forecast actual expenditures.

2. The cost of remedy reviews would be included in those for the LAOCB OU, depending on the remedy selected for the LAOCB.

$\mathrm{S} / \mathrm{S}=$ Stabilization/Solidification 


\subsection{INTRODUCTION}

This Corrective Measures Study (CMS)/Feasibility Study (FS) report has been prepared for the L-Area Oil and Chemical Basin (LAOCB) at the Savannah River Site (SRS) near Aiken, South Carolina. The LAOCB, or Basin, was designed and constructed as an unlined seepage basin for the purpose of disposing of small volumes of wastes that were not appropriate for discharge to local streams or the waste management system. The LAOCB Solid Waste Management Unit/Operable Unit (SWMU/OU) includes the Basin soil/sediment, soil adjacent to the unit, the pipelines and associated soil, vegetation, groundwater, and the L-Area Acid/Caustic Basin (LAACB). However, contamination at the LAACB is related to acid/caustic solutions laden with non-radioactive metals and non-metals; only low concentrations of naturally occurring radionuclides are present at the LAACB. Additionally, no impact to groundwater by these solutions has occurred at the LAACB. Because of the nature of contamination and the findings of the Baseline Risk Assessment (BRA), it has been determined that the risk is very low at the LAACB, the LAACB pipeline and drainage ditch, and the area adjacent to the LAOCB. A No Action option is appropriate for these components of the OU. Further characterization of the soils associated with the LAACB will be performed to confirm $\mathrm{pH}$ values as identified in the Resource Conservation and Recovery Act (RCRA) Facility Investigation (RFI)/Remedial Investigation (RI). Therefore, contaminated materials associated with LAACB are not addressed in this report.

The groundwater at the OU will be addressed following additional characterization as identified in the RFI/RI. Final remedial activities for groundwater will be developed following the characterization. The results of this RFI/RI and its associated BRA and RFI/RI documentation provides sufficient information to move forward with a focused CMS/FS, Proposed Plan, Interim Action - Record of Decision (IA-ROD), and remedial action of the surface and vadose zone components of unit. However, this investigation recognized that the horizontal extent of groundwater contamination had not been completely characterized downgradient of the LAOCB 
and in areas adjacent to the L-Area Hot Shop and LAOCB. Also, any remedial actions directed toward the groundwater at this time could cause commingling of contamination from the Hot Shop and L-Reactor Seepage Basin, resulting in a larger problem than currently exists. Therefore, the extent of groundwater contamination adjacent to the LAOCB, the L-Area Hot Shop, and the L-Reactor Seepage Basin must be defined prior to any final groundwater remedial action plans for the area. For additional documentation, refer to the RFI/RI Report (WSRC, 1996a). The RFI/RI provides the phased approach plan for designating further characterization of the groundwater of the LAOCB, and the Hot Shop to be conducted for the waste units in the vicinity of the LAOCB. This characterization would be followed by a CMS/FS, Proposed Plan, and IA-ROD as conducted for the surface and vadose zone components of the LAOCB and LAACB. In the event that earlier interim actions on the groundwater and/or soils are feasible, then these actions could be integrated into the plan and associated documentation.

Liquid waste consisting of small volumes of slightly radioactive oil and chemical wastewater were sent to the LAOCB from various waste streams throughout SRS, but primarily from the reactor areas. Wastewater flowed into the Basin from a bermed concrete drainage pad that was located outside the Basin perimeter fence, and from an underground pipeline originating at the maintenance Hot Shop (Building 717-G, referred to as the Hot Shop in this document). Wastes were transported to the drainage pad in tank trucks, metal drums, skid tanks, and other containers. The Hot Shop discharged decontamination wastewater containing radionuclides, detergents, and spent degreasing solvents through the pipeline to the Basin. Constituents of concern $(\mathrm{COC})$ within the LAOCB include radionuclides and metals.

Sections 1.1 and 1.2 define the purpose and organization of this CMS/FS Report. Section 1.1 also includes a description of the RCRA/Comprehensive Environmental Response, Compensation, and Liability Act (CERCLA) Program at SRS. Section 1.3 provides background information on the LAOCB obtained from the RFI and the CERCLA RI programs. 


\subsection{Purpose}

The purpose of the CMS/FS is to develop remedial action objectives; identify, screen, and evaluate remedial technologies considered applicable to the unit-specific conditions; and develop and evaluate appropriate corrective measures/remedial alternatives. The approach adopted to achieve the CMS/FS objectives is stated in Section 1.2. A description of the RCRA/CERCLA Program at SRS and the role of the CMS/FS in SRS program activities appears below.

\subsubsection{SRS RCRA 3004(u)/CERCLA Program Description}

The U.S. Department of Energy (DOE) Savannah River Operations Office manages waste materials which are regulated under RCRA. In particular, certain activities have required operating or post-closure permits under RCRA. The SRS has received a RCRA permit from the South Carolina Department of Health and Environmental Control (SCDHEC). Part V of the permit mandates that SRS establish and implement an RFI Program, in order to fulfill requirements of RCRA Section 3004(u). Section V.A.1 of the permit lists 65 solid waste management units (SWMUs) that were identified by the Environmental Protection Agency (EPA) Region IV through the RCRA Facility Assessment (RFA) process. A requirement of the permit mandates that these 65 SWMUs be further investigated to determine their actual or potential impact on the environment.

Prior to conducting the investigations, SRS submitted the RFI Program Plan (WSRC, 1989) which specifies the generic methods and procedures to be used for each unit investigation. This Program Plan has been developed to provide guidance and delineate standard procedures for facility investigations. The Program Plan provides a reasonable and cost effective approach while allowing SRS flexibility in the investigation of each unit. 
On December 21, 1989, SRS was included on the National Priorities List (NPL). A site included on the NPL falls under the jurisdiction of CERCLA. This created the need to integrate the established RFI Program with CERCLA requirements. In accordance with Section 120 of CERCLA, the DOE has negotiated a Federal Facility Agreement (FFA) (WSRC, 1993a) with EPA and SCDHEC to integrate remedial activities at the SRS into one comprehensive strategy, which fulfills these dual regulatory requirements. The RFI Program Plan was subsequently revised to integrate the CERCLA RI with RCRA 3004(u) requirements and was published as the RFI/RI Program Plan (WSRC, 1993b). Since approval of the initial Program Plan by the EPA, SRS has added a number of newly-identified units to the original list of 65 SWMUs (termed "RCRA/CERCLA units" in this document).

The LAOCB is one of the RCRA/CERCLA units listed in Appendix C of the FFA (WSRC, 1993a) and mandated for further investigation. As a result, a RFI/RI was conducted at this unit. The RFL/RI Program Plan and Guidance for Conducting Remedial Investigations and Feasibility Studies Under CERCLA, Interim Final (EPA, 1988a) were used as the primary sources of guidance for RFI/RI work plan and report preparation. These references were jointly referred to as RFI/RI guidance documents. The work plan and the RFIRI Report (WSRC, 1996a) for the LAOCB conformed to the requirements specified in the RFI/RI guidance documents.

A Baseline Risk Assessment (BRA) was conducted using data generated during the RFI/RI Unit Assessment. The BRA evaluated potential risks posed by the unit to human health and the environment. The BRA is summarized in Section 6.0 of the RFI/RI report and was submitted as a separate document (WSRC, 1996b).

This CMS/FS Report was conducted in accordance with the requirements contained in the RFI/RI guidance documents. This document is referred to as a CMS/FS since it satisfies both RCRA CMS and CERCLA FS requirements. This report was based on the data, results, and 
conclusions contained in two previous RCRA/CERCLA documents, the LAOCB RFI/RI Report and the LAOCB BRA.

Public participation requirements are listed in Sections 113 and 117 of CERCLA. These requirements include the establishment of an Administrative Record File that documents the selection of cleanup alternatives and provides for review and comment by the public on those alternatives. An SRS Public Involvement Plan (PIP) (DOE, 1994a) is designed to facilitate public involvement in the decision-making processes for permitting, closure, and the selection of remedial alternatives. The PIP addresses the requirements of CERCLA, RCRA, and the National Environmental Policy Act (NEPA).

Unit-specific documentation, such as work plans and reports, will be a part of the Administrative Record File and will be available to the public. Information repositories have been established at DOE's Public Reading Room, located in the Gregg-Graniteville Library of the University of South Carolina in Aiken, South Carolina, and the Government Documents Department of the Thomas Cooper Library located at the University of South Carolina in Columbia, South Carolina. A notice will be published in local newspapers when information is being compiled regarding the investigation and cleanup of the LAOCB and LAACB. Additional repositories may be added and/or locations changed to better meet the needs of the public.

\subsection{Approach and Report Organization}

The FS was developed in accordance with CERCLA guidance as promulgated under the National Oil and Hazardous Substances Contingency Plan (NCP) of November 20, 1985 (50 FR 47973), the Superfund Amendments and Reauthorization Act (SARA) of October 17, 1986, and the amended NCP of March 5, 1990 (55 FR 8666). The general framework of this report is based on the EPA document Guidance for Conducting Remedial Investigations and Feasibility Studies Under CERCLA (EPA, 1988a), the DOE document Remedial Investigations/Feasibility Study 
Process, Elements, and Techniques Guidance (DOE, 1993), and the Westinghouse Savannah River Company (WSRC) document RFI/RI Program Plan, Revision 1 (WSRC, 1993b).

The CMS was developed in accordance with RCRA guidance as enacted under the Hazardous and Solid Waste Amendments (HSWA) of 1984; however, regulations establishing the framework for implementation of the HSWA provisions have not yet been promulgated. EPA proposed regulations on July 27, 1990 (55 FR 30798). HSWA gave EPA authority to require corrective actions for all releases of hazardous waste or constituents from any SWMU at facilities regulated under a RCRA permit. The proposed regulations establish a framework similar to the revised NCP. The EPA document RCRA Corrective Action Plan (EPA, 1988b) was used as a guide in preparing the CMS. Additionally, RCRA CMS aspects of the WSRC document referenced above were used in the development of this CMS/FS.

The CMS/FS for the LAOCB has utilized a focused approach which is limited to the Basin area, the area adjacent to the Basin, and the pipeline area. The groundwater medium and the LAACB are not addressed as part of this CMS/FS. The only concern for the LAACB is pH. Further testing will be conducted and a No Action ROD will be pursued. Additional investigation of the sources of groundwater contamination at the LAOCB will be conducted. However, this is a complete FS for the LAOCB.

Figure 1-1 provides a flowchart diagram of the CMS/FS process. The basic steps in the CMS/FS process and, consequently, the organization of this CMS/FS report are as follows:

- summarize the results of the RFI/RI, including the BRA (Section 1.3)

- identify the media of concern that will be addressed in this CMS/FS (Section 2.2)

- $\quad$ determine the remedial action objectives for the media of concern (Section 2.3)

- identify the general response actions that may be used to address the media of concern (Section 2.4) 
- identify remedial technologies under each general response action that may have potential application under unit-specific conditions (Section 2.5)

- screen potential remedial technologies based on their effectiveness, implementability and cost (Section 2.6)

- develop potential media-specific remedial alternatives (Section 3.1)

- $\quad$ screen media-specific remedial alternatives based on effectiveness, implementability, and cost (Section 3.2)

- identify comprehensive corrective measures/remedial alternatives that address the entire LAOCB waste unit (Section 3.3)

- conduct a detailed analysis of potential corrective measures/remedial alternatives based on NCP and RCRA criteria (Section 4.2)

- conduct a comparative analysis of the corrective measures/remedial alternatives (Section 4.3)

Although a comparative analysis of corrective measures/remedial alternatives is presented in Section 4.0, this CMS/FS does not propose a preferred alternative. The preferred alternative for the LAOCB will be presented in the Statement of Basis/Proposed Plan to be submitted under separate cover. Selection of the preferred alternative in the Statement of Basis/Proposed Plan will be based on information contained in the LAOCB RFI/RI and CMS/FS reports and EPA, SCDHEC, and public acceptance.

\subsection{Background Information}

The following subsections provide background information obtained from the LAOCB RFI/RI report (WSRC, 1996a). 


\subsubsection{Unit Description}

SRS is located principally in Barnwell and Aiken Counties approximately 32 kilometers $(\mathrm{km})$ [20 miles (mi)] south of the city of Aiken, South Carolina (Figure 1-2). The locations of major SRS facilities are also shown in Figure 1-2.

\subsubsection{Surface Features and Topography}

The topographic setting of the unit is shown in Figure 1-3. The LAOCB is located in an area of low to moderate relief just outside and south of the L-Area perimeter fence. It is located on the southern flank of a hill approximately 380 meters (m) [(1,250 feet (ft)] north of $\mathrm{L}$ Lake on the south side of the divide between L Lake and Pen Branch. The area lies at an elevation of

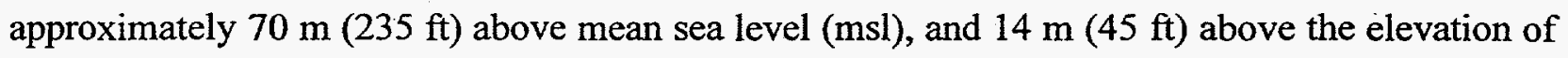
L Lake. A Carolina Bay (Bay 116) is located approximately $1,520 \mathrm{~m}(5,000 \mathrm{ft})$ northeast and upgradient of the Basin.

The LAOCB measures $55 \mathrm{~m}$ (182 ft) long and $33 \mathrm{~m}(108 \mathrm{ft})$ wide at the berm with an

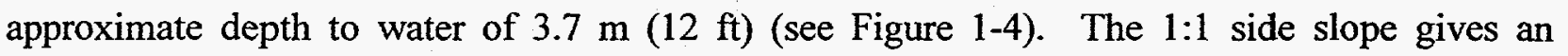
approximate freeboard capacity of $4,206 \mathrm{~m}^{3}\left(148,500 \mathrm{ft}^{3}\right)$. Depth from equivalent ground surface grade to the top of the sediment is about 3.1 to $4.0 \mathrm{~m}$ (10 to $13 \mathrm{ft}$ ); about 15.2 centimeters $(\mathrm{cm})$ [6 inches (in.)] of sludge/sediment are present in the Basin. Water in the Basin is primarily from rainfall. Some water is present in the Basin at most times due to rainfall; however, the Basin has been observed to be dry during dry weather conditions. The Basin has contained up to $0.9 \mathrm{~m}(3$ $\mathrm{ft})$ of water during prolonged periods of precipitation. 


\subsubsection{Ecology}

General ecological reconnaissance or surveys were conducted in November 1992 and April 1994. Files of the Savannah River Forest Service (SRFS) were examined for the LAOCB site. Detailed descriptions of the plant and wildlife communities observed is contained in the RFI/RI Report for the LAOCB (WSRC, 1996a).

A Threatened, Endangered, or Sensitive (TES) Species survey was conducted at the LAOCB by the SRFS of the U.S. Forest Service in May 1993 (Jarvis, 1993). SRFS files were examined to determine past or present occurrences of any TES species at or in the vicinity of this unit. Records reviewed included aerial photographs, vegetative stand data, soil information, and existing TES species records. This review was followed by a field survey to document any TES plant or animal species potentially occurring at the unit based on past recorded observations or presence of required habitat elements. The survey was conducted within a study area of approximately 0.8 hectare (ha) [2 acres (ac)], which encompasses the Basin and the fenced area around the Basin. The Basin itself covers an area of $0.1 \mathrm{ha}(0.3 \mathrm{ac})$ and is surrounded by a fence. The area outside the fence is covered by mowed grass.

Neither the May 1993 survey nor other current or historic records indicates the occurrence of any TES plant or animal populations/individuals in the study area. Those TES species considered to have the potential to inhabit the study area based on their habitat requirements and ranges are listed in Table 1-1 together with Federal and State Heritage Trust Program status rankings (Jarvis, 1993). Of the TES animal species listed, only the Carolina gopher frog might reasonably be expected to utilize habitat provided by the Basin. Of the TES plant species listed, those which utilize bay/ditch/pond/meadow habitats have some potential to occur within the Basin. Habitation or utilization of the Basin by other TES plant/animal species listed is highly unlikely. 


\subsubsection{Meteorology}

The regional climate at SRS is characterized by warm humid summers and short mild winters. The average annual temperature is $17^{\circ} \mathrm{C}\left(64^{\circ} \mathrm{F}\right)$, with monthly average temperatures ranging from $7^{\circ} \mathrm{C}\left(45^{\circ} \mathrm{F}\right)$ in January to $27^{\circ} \mathrm{C}\left(87^{\circ} \mathrm{F}\right)$ in July.

The average annual precipitation at SRS is $122 \mathrm{~cm}$ (48 in.). Precipitation is distributed fairly evenly throughout the year, with the highest precipitation in summer $(36.1 \mathrm{~cm} ; 14.2 \mathrm{in}$.) and lowest in fall $(22.4 \mathrm{~cm} ; 8.8 \mathrm{in}$.). Snowfall is rare, occurring at an annual average of $3.0 \mathrm{~cm}(1.2$ in.). Approximately 73 percent of the average annual precipitation is lost to evapotranspiration. The remaining precipitation represents the amount available to surface streams for overland flow and to groundwater for recharge.

\subsubsection{Geology}

\section{Regional Geology}

A detailed discussion of regional geology, including descriptions of stratigraphic units, is contained in the LAOCB RFI/RI Report (WSRC, 1996a).

\section{$\underline{\text { Unit-Specific Geology }}$}

A shallow, sandy clay to clayey sand unit, under the LAOCB area designated the local "hardpan" zone, occurs at a typical depth of 3 to $4 \mathrm{~m}$ (10 to $13 \mathrm{ft}$ ) below land surface (bls) at an approximate elevation of $67 \mathrm{~m}(220 \mathrm{ft})$ mean sea level $(\mathrm{msl})$ under the LAOCB area. The unit's thickness varies from less than $0.3 \mathrm{~m}(1 \mathrm{ft})$ to more than $1.5 \mathrm{~m}(5 \mathrm{ft})$, increasing southeastward under the Basin. The hardpan unit strike trends northeast-southwest. The hardpan thins and 
pinches out northwest of the LAOCB. Thickness of the hardpan in the pipeline vicinity is $0.3 \mathrm{~m}$ (1 ft) or less. Thickness increases toward the southeast.

The hardpan zone varies from a clayey sand to sandy clay facies. The clay is dry, with ironoxide concretions, and color mottled by iron-oxide staining. The iron-oxide concretions observed at the LAOCB appear typical of iron-cemented concretions observed at other locations in Aiken County.

The hardpan zone consists of three general facies: a sand/silt facies, a clayey sand facies, and a clay/sandy clay facies. The sand/silt facies occurs to the northwest of the $\mathrm{LAOCB}_{2}$ and is present in the areas of the pipeline breaks. The sandy nature of this facies likely provides relatively greater potential for downward movement of liquids compared to the clayey sand and clay/sandy clay facies. The clayey sand facies occurs to the south and southeast of the LAOCB. This facies likely provides intermediate potential for downward movement of liquids compared to the two other facies. The clay/sandy clay facies occurs under the LAOCB, particularly under the southeast portion of the Basin. The clay/sandy clay facies is located between the sand/silt facies to the north and west, and the clayey sand facies to the north and east. The clay/sandy clay facies in the vicinity of the LAOCB likely provides the greatest confining characteristics of the three hardpan facies.

A clayey zone, designated as "AA" horizon, is found at a typical depth of 4.9 to $5.5 \mathrm{~m}$ (16 to 18 $\mathrm{ft}) \mathrm{bls}$ with an average elevation of $66 \mathrm{~m}(215 \mathrm{ft}) \mathrm{msl}$. The "AA" horizon thickness, when present, increases from less than $0.3 \mathrm{~m}(1 \mathrm{ft})$ in the pipeline area and the area south of LAOCB to more than $3 \mathrm{~m}(10 \mathrm{ft})$. The thicker, southeastern portion of the clayey zone includes an interbedded sand, sandwiched between finer-grain " $\mathrm{AA}$ " horizon zones. The "AA" horizon strike trends northeast-southwest, with dip to the southeast. The "AA" horizon surface maximum relief is 1.8 to $2.5 \mathrm{~m}$ ( 6 to $8 \mathrm{ft}$ ). The general slope of the top of the horizon downward to the southeast is marked by an elevationally high area that coincides with the thickest portion of the horizon. A 
contrasting low area is located approximately $30 \mathrm{~m}(100 \mathrm{ft})$ southeast of the LAOCB. The "AA" horizon's fine-grain nature likely results in a relatively lower potential for downward movement of liquids compared to the under- and overlying coarser-grain zones.

A clayey unit, designated as "A" horizon is identified at a typical depth of 6.4 to $9.7 \mathrm{~m} \mathrm{(21}$ to 32 $\mathrm{ft})$ bls with an approximate elevation of $62 \mathrm{~m}(205 \mathrm{ft}) \mathrm{msl}$. The "A" horizon thickness varies from less than $0.3 \mathrm{~m}(1 \mathrm{ft})$ in the pipeline vicinity, northeast of the Basin to more than $1.5 \mathrm{~m}(5$ $\mathrm{ft}$ ) in the southern LAOCB area. Thickness also decreases from these areas of greatest thickness

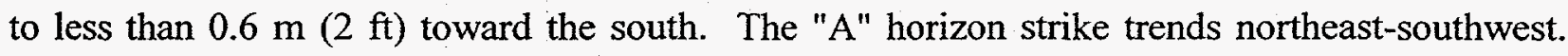
Unit dip is to the southeast. The " $\mathrm{A}$ " horizon clay is firm to stiff, moist, and mottled. The mottling colors are moderate-reddish brown and grayish-red purple. Horizon " $\mathrm{A}$ " appears to be part of the lowest portion of the overlying, mottled Tobacco Road Formation, and is underlain by the generally sandier Dry Branch Formation. The thinning of the " $\mathrm{A}$ " horizon in the pipeline vicinity is coincident with the thinning of the "AA" horizon and the sand/silt facies of the hardpan zone. This combination of lithologic traits increases the potential for more rapid downward liquid movement in the area of the pipeline breaks.

A relatively thick 1.5 to $8 \mathrm{~m}$ ( 5 to $26 \mathrm{ft}$ ) zone of interbedded to interlaminated sands, silts, and clays is identified below the "AA" unit and above and/or below the " $\mathrm{A}$ " unit in the study area. The top of the zone typically occurs at depths between 8.2 to $10 \mathrm{~m} \mathrm{(27} \mathrm{to} 33 \mathrm{ft}$ ) bls. The zone is identifiable over the entire study area.

The "B" horizon is a sand unit. The unit occurs at a typical depth of 11 to $17 \mathrm{~m} \mathrm{(35} \mathrm{to} 55 \mathrm{ft}$ ) bls at an approximate elevation of $57 \mathrm{~m}(187 \mathrm{ft}) \mathrm{msl}$. The "B" horizon extends downward to the "Tan Clay" interval, for an approximate thickness of 4.6 to $11 \mathrm{~m}$ (15 to $35 \mathrm{ft}$ ). These sands are probably Dry Branch Formation sediment. They are usually described as a silty/clayey sand, pale red, yellow orange or tan to brown. Underlying this silty/clayey sand interval is an interval which is sandier. The "B" horizon is continuous across the study area. 
Within the sandy "B" horizon, an interval is described as a clayey sand beginning at a depth of $20.4 \mathrm{~m}(67 \mathrm{ft})$ bls at an approximate elevation of $52 \mathrm{~m}(170 \mathrm{ft}) \mathrm{msl}$. The " $\mathrm{C}$ " horizon is traceable at 50 to $55 \mathrm{~m}$ (165 to $180 \mathrm{ft}$ ) $\mathrm{msl}$, and represents a clayey interval in an otherwise relatively clean sandy zone. The sandy "B" horizon resumes below the clayey " $\mathrm{C}$ " horizon, extending downward 4.6 to $6.1 \mathrm{~m}$ (15 to $20 \mathrm{ft})$ to the "Tan Clay" interval which marks the bottom of the water table aquifer (Aquifer Zone $\mathrm{IIB}_{2}$ ).

\subsubsection{5 $\underline{\text { Soil }}$}

The soil in the immediate vicinity of the LAOCB are classified as Udorthent (USDA, 1990). This is a generic term indicating that the natural soil weathering horizons have been disturbed or removed, usually by construction activities or erosion. Soil may also be classified as Udorthent when either the topsoil is removed and/or disturbed soil is used for fill activities during grading for parking lots or to create level areas, as was the case during construction of the LAOCB. Evaluation of the soil survey of SRS (USDA, 1990) indicates that prior to construction activities at L Area, the soil in the LAOCB area were probably Fuquay or Dothan series. Both these soil series characteristically have perched water tables at 0.9 to $1.8 \mathrm{~m} \mathrm{(3} \mathrm{to} 6 \mathrm{ft}$ ) bls, from January through April (USDA, 1990).

\subsubsection{Hydrogeology}

\section{$\underline{\text { Regional Hydrogeology }}$}

A detailed discussion of regional hydrogeology, including hydrostratigraphic nomenclature, is contained in the LAOCB RFI/RI Report (WSRC, 1996a). 


\section{Local/Site Hydrogeology}

Water level data reveals that the water table surface ranges in depth from 4 to $8 \mathrm{~m}$ (12 to $25 \mathrm{ft}$ ) and with elevations from 70 to $74 \mathrm{~m}$ (230 to $244 \mathrm{ft}) \mathrm{msl}$. The water table aquifer extends to the top of the "Tan Clay" interval. The water table aquifer is approximately $17 \mathrm{~m}(55 \mathrm{ft})$ thick (vicinity of LCO-5). The water table can be divided into upper and lower aquifers. The upper water table consists primarily of aquifer zone I. Aquifer zone IIa is also included in the upper water table. The lower water table consists of aquifer zone IIb and aquifer zone III.

Groundwater flow direction for the water table surface is south-southeast and south-southwest for aquifer zone IIb. Horizontal gradients of 0.011 and $0.013 \mathrm{ft} / \mathrm{ft}$ were calculated for the water table surface and aquifer zone $\mathrm{IIb}$ potentiometric surface, respectively.

Hydraulic conductivities for aquifer zone I and zone IIa sediment ranged from 5.65E-5 to $4.30 \mathrm{E}-3 \mathrm{~cm} /$ second $(0.16$ to $12.17 \mathrm{ft} /$ day) and averaged $9.25 \mathrm{E}-4 \mathrm{~cm} / \mathrm{second}(2.62 \mathrm{ft} /$ day). The lateral seepage (pore) velocity for the upper water table is estimated to be 0.56 to $42.53 \mathrm{~m} /$ year (1.83 to $139.44 \mathrm{ft} /$ year).

Estimated hydraulic conductivities for aquifer zone IIb sediment range from $4.24 \mathrm{E}-5$ to $9.28 \mathrm{E}-4$ $\mathrm{cm} /$ second ( 0.12 to $2.63 \mathrm{ft} /$ day) and average $2.78 \mathrm{E}-4 \mathrm{~cm} / \mathrm{second}(0.79 \mathrm{ft} /$ day $)$. The calculated lateral seepage (pore) velocity for aquifer zone IIb ranges from 0.50 to $10.80 \mathrm{~m} /$ year (1.64 to $35.4 \mathrm{ft} /$ year). Hydraulic conductivities of aquifer zone $\mathrm{IIB}_{1}$ (McBean) and aquifer unit IIA (Congaree) are $3.08 \mathrm{~m} /$ day $(10.11 \mathrm{ft} /$ day) and $0.03 \mathrm{~m} /$ day $(0.11 \mathrm{ft} /$ day), respectively.

Horizontal permeability for the clay ranges from $2.2 \mathrm{E}-7$ to $3.0 \mathrm{E}-6 \mathrm{~cm} /$ second $(6.23 \mathrm{E}-4$ to $8.49 \mathrm{E}$ $3 \mathrm{ft} /$ day) with an average of $1.47 \mathrm{E}-6 \mathrm{~cm} / \mathrm{second}(4.16 \mathrm{E}-3 \mathrm{ft} /$ day). The vertical permeability ranges from $1.5 \mathrm{E}-7$ to $6.0 \mathrm{E}-7 \mathrm{~cm} / \mathrm{second}(4.25 \mathrm{E}-4$ to $1.7 \mathrm{E}-3 \mathrm{ft} /$ day), with an average of $3.67 \mathrm{E}-7$ $\mathrm{cm} /$ second (1.04E-3 ft/day). 
Recharge to the upper water table is from net rainfall infiltration and to a lesser extent by inflow from upgradient inflow. Aquifer zone IIa receives recharge from leakage across horizon "A", especially in the area where thinning of the "AA" and " $\mathrm{A}$ " horizons occur in the vicinity of the pipeline. Discharge from aquifer zone I is downgradient to the south-southeast toward L Lake.

Aquifer zone IIb receives recharge from leakage across the interbedded sands, silts, and clays and from upgradient inflow. Discharge is toward L Lake to the south-southwest.

\subsubsection{Surface Water Hydrology}

Surface water runoff in $\mathrm{L}$ Area drains southward to $\mathrm{L}$ Lake through overland flow and small intermittent stream channels and drainage ditches. A road ditch (for SRS Road-B) and culvert convey overland flow from L Area to a canal that empties into L Lake.

Wastewater has never been reported to have overflowed from the LAOCB. Sufficient freeboard volume exists $\left(4158 \mathrm{~m}^{3} ; 148,500 \mathrm{ft}^{3}\right)$ to ensure that an overflow event is unlikely.

A berm surrounding the Basin diverts overland flow away from the Basin. Direct precipitation is currently the only source for Basin water. Overflow from the LAOCB would drain southward to L Lake as described above; however, due to the significant freeboard in the Basin, no overflow is expected.

\subsubsection{Unit History}

Prior to construction of SRS, the area that is now referred to as L Area was used for agricultural purposes. An aerial photograph from October 17, 1951, records the presence of a farmhouse, miscellaneous outbuildings, and a corral just south of where LAOCB is now located. The 
photograph illustrates that the Basin was constructed in an open field north of the farmhouse and outbuildings.

The LAOCB was designed and constructed as an unlined seepage basin for the purpose of disposing of small volumes of wastes that were not appropriate for discharge to local streams, regular seepage basins, or the waste management system.

The Basin was put in operation in 1961 and remained active until 1979. Rainfall has resulted in the presence of some water in the Basin at most times. During October 1988, when the unit reconnaissance was conducted, the Basin was dry and exhibited desiccation features in the bottom sediment. During the November 1992 reconnaissance, the Basin contained approximately one-half to one foot of rainwater.

Liquid waste consisting of small volumes of slightly radioactive oil and chemical wastewater were sent to the LAOCB from throughout SRS, but came primarily from the reactor areas. Wastewater flowed into the Basin from a bermed concrete drainage pad that was located outside the Basin perimeter fence, and from an underground pipeline originating at the maintenance Hot Shop (Building 717-G, referred to as the Hot Shop in this document). Wastes were transported to the drainage pad in tank trucks, metal drums, skid tanks, and other containers. The Hot Shop. discharged decontamination wastewater containing radionuclides, detergents, and spent degreasing solvents through the pipeline to the Basin.

Historical records indicate that wastes from all sources contributed 2.2 curie ( $\mathrm{Ci}$ ) of alpha emitters and $270 \mathrm{Ci}$ of nonvolatile beta emitters including $0.1 \mathrm{Ci}$ of strontium-90 $\left({ }^{90} \mathrm{Sr}\right)$ and 0.4 $\mathrm{Ci}$ of cesium-137 ( $\left.{ }^{137} \mathrm{Cs}\right)$. (Fenimore et al., 1988). A small undocumented amount of radioactivity was released to the Basin through infrequent repair work in the Hot Shop. Several filters at the L-Reactor building's distillation and purification system had high external radiation exposure rates, and underwater work was performed on the filters to protect personnel. A tank 
filled with water was placed inside the Basin perimeter fence and used for shielding during work on the filters. After repairs to the filters were completed, including disassembly/assembly, the water was drained to the Basin, and the tank was removed from the area. Although records indicate that radioactivity was released to the Basin through this activity, the chemical composition of the releases is not known.

\subsubsection{Conceptual Unit Model}

A conceptual unit model is used to simplify the breadth of information that is gathered during unit investigations into an ordered set of components that can be used to guide further investigations, technology screening, remedial alternatives evaluation, and selection of the final remedy. Components of a conceptual unit model include: summary descriptions of the source, primary contaminated media, migration pathways, exposure pathways, potential receptors, and COCs.

The conceptual unit model for the LAOCB is based on the data that are presented in the RFI/RI report (WSRC, 1996a). For this CMS/FS report the model considers only source sludge and subsoil in the LAOCB, soil outside the LAOCB, the LAOCB pipelines and associated soil, and contaminated vegetation. Specifically, contaminated media addressed in this report are:

- $\quad$ LAOCB sludge and subsoil

- $\quad$ soil adjacent to the LAOCB

- $\quad$ LAOCB pipelines soil

- vegetation (in the immediate vicinity of the LAOCB)

A summary of potential receptors, exposure pathways, and COCs for each of these media for which contaminants posed potential risks or hazards is provided in Figures 1-7a through $-7 \mathrm{~h}$. 
These components of the conceptual unit model are consistent with the results of the EPA and SCDHEC approved RFI/RI and BRA.

\subsubsection{Nature and Extent of Contamination}

The RFI/RI was conducted in accordance with the RFI/RI Work Plan (WSRC, 1993c). The following presents a summary of the results of the RFI/RI program in regards to Basin contamination.

\subsubsection{Summary of Nature and Extent of Contamination in the LAOCB}

This section summarizes the nature and extent of contamination in standing water, sludge/subsoil, and soil in and around the Basin and along the LAOCB pipeline. Contamination of vegetation is also summarized in this section. Contaminants detected within each medium are listed in Table 1-2. Further discussion of the nature and extent of contamination appears in the LAOCB RFI/RI Report (WSRC, 1996a).

\section{$\underline{\text { Source Area Sludge and Subsoil }}$}

The source area is defined as the Basin. Sampling of the Basin sludge and subsoil indicate that the sludge within the Basin is approximately $15 \mathrm{~cm}$ (6 in.) thick. A summary of contaminants detected in the Basin sludge and subsoil is included as Table 1-3. The nature and extent of contamination in source area sludge and subsoil can be summarized as follows:

- Radionuclide species: The sludge within the LAOCB contains the highest concentrations (or levels) of radionuclides at the unit. The subsoil sampled below the sludge are also contaminated with radionuclides; however, the concentrations of radionuclides decrease rapidly with depth. The major radionuclides with respect to activity within the Basin 
sludge and subsoil that are anthropogenic are: Americium-241 $\left({ }^{241} \mathrm{Am}\right),{ }^{137} \mathrm{Cs}$, cobalt-60 $\left({ }^{60} \mathrm{Co}\right)$, Europium-152 $\left({ }^{152} \mathrm{Eu}\right),{ }^{154} \mathrm{Eu}$, plutonium-238 $\left({ }^{238} \mathrm{Pu}\right),{ }^{239} \mathrm{Pu},{ }^{90} \mathrm{Sr}$, uranium-234 $\left({ }^{234} \mathrm{U}\right),{ }^{235} \mathrm{U},{ }^{238} \mathrm{U}$, thorium-234 $\left({ }^{234} \mathrm{Th}\right)$, and tritium $\left({ }^{3} \mathrm{H}\right)$.

- The average activity for the Basin sludge and subsoil [to a depth of $0.5 \mathrm{~m}(1.5 \mathrm{ft})$ ] is $3,833.3$ picocuries per gram (pCi/g) for the major radionuclides (i.e., ${ }^{241} \mathrm{Am},{ }^{137} \mathrm{Cs},{ }^{60} \mathrm{Co}$, ${ }^{152} \mathrm{Eu},{ }^{154} \mathrm{Eu},{ }^{238} \mathrm{Pu},{ }^{239} \mathrm{Pu},{ }^{90} \mathrm{Sr},{ }^{235} \mathrm{U},{ }^{238} \mathrm{U}$, and $\left.{ }^{3} \mathrm{H}\right)$. The total radionuclide activity within the Basin [including the sludge and subsoil to a depth of $0.5 \mathrm{~m}(1.5 \mathrm{ft})$ ] is approximately 4.2 curies. An analysis of the attenuation of the maximum gross nonvolatile beta and alpha emissions indicate that radionuclide concentrations (other than ${ }^{3} \mathrm{H}$ ) should reach background activity levels within two feet from the top of the sludge. Linear regression analysis of the gross alpha values $(\log )$ versus sample depth demonstrates that the maximum observed sludge gross alpha values decrease to activities less than background levels (WSRC, 1995a) at a depth of $0.5 \mathrm{~m}$ (1.5 ft) from the top of the sludge (Figure 1-5). Linear regression analysis of the gross nonvolatile beta values (log) versus sample depth demonstrates that the maximum observed sludge gross nonvolatile beta values decrease to activities less than background levels at a depth of $0.6(2 \mathrm{ft})$ from the top of the sludge (Figure 1-6; WSRC, 1995a).

- A review of the ${ }^{3} \mathrm{H}$ activities of the Basin sludge and subsoil indicates that ${ }^{3} \mathrm{H}$ activities also decrease rapidly with depth. The maximum ${ }^{3} \mathrm{H}$ activity within the Basin sludge is $15,498 \mathrm{pCi} / \mathrm{g}$; however, the maximum ${ }^{3} \mathrm{H}$ activity at $0.3 \mathrm{~m}(1 \mathrm{ft})$ below the Basin sludge is $137.9 \mathrm{pCi} / \mathrm{g}$.

- Metals: Seventeen metals were detected in the LAOCB sludge and subsoil samples at concentrations exceeding screening levels (Table 1-3). Relatively high concentrations of chromium $(\mathrm{Cr})$, beryllium $(\mathrm{Be})$, copper $(\mathrm{Cu})$, cadmium $(\mathrm{Cd})$, lead $(\mathrm{Pb})$, and zinc $(\mathrm{Zn})$ are attributed to decontamination of stainless steel, galvanized metals, and brass. All 17 
metals, except barium $(\mathrm{Ba})$, cobalt $(\mathrm{Co}), \mathrm{Cu}$, mercury $(\mathrm{Hg})$, and $\mathrm{Zn}$, exceed risk-based Preliminary Remediation Goals (PRGs) in at least one sample.

- Organic constituents: Four volatile organic compounds (VOCs) were detected above screening levels (Table 1-3) in the LAOCB sludge or subsoil at frequencies of less than 50 percent. These compounds are methyl-ethyl-ketone (MEK), ethylbenzene, carbon disulfide, and toluene. All VOCs but MEK were determined to be false positives. Reported MEK concentrations are near the screening level and well below risk-based PRGs.

- No analyses were conducted for semi-volatile organic compounds (SVOCs̄).

- The LAOCB sludge/subsoil contains total petroleum hydrocarbons (TPH) (full range) with a median observed concentration of 11.34 milligrams per kilogram $(\mathrm{mg} / \mathrm{kg})$, and a maximum observed concentration of $7,186 \mathrm{mg} / \mathrm{kg}$. No apparent pattern to the distribution of petroleum hydrocarbons in the Basin is evident.

- Based on the waste disposal history of the LAOCB, pesticides, polychlorinated-biphenyl (PCB), dioxins, and furans were probably not released to the Basin during its operations, and were, therefore, not analyzed.

\section{Soil Adjacent to the LAOCB}

A summary of contaminants detected in the soil adjacent to the LAOCB is included as Table 1-4. The nature and extent of contamination in the soil adjacent to the LAOCB can be summarized as follows: 
- Radionuclide species: Based on field screening results, it is evident that there is no manmade radionuclide contamination of soil adjacent to the LAOCB. The detected radionuclides are determined to be strictly naturally occurring.

- Metals: Eighteen metals are reported at concentrations above screening values in the soil samples collected adjacent to the LAOCB (Table 1-4).

- Organics constituents: Six VOCs and one SVOC were detected at concentrations exceeding screening values in soil samples from locations adjacent to the LAOCB. Twenty-six compounds were detected in some samples at very low concentrations. Each of the 26 compounds detected are either laboratory artifacts, present in concentrations below the quantitation limit, or detected at low concentrations above quantitation limits. No apparent vertical or lateral trends in these data that may indicate concentration gradients or hot spot areas were noted.

- Soil were not analyzed for TPH because TPH compounds were not expected to migrate beyond the Basin sludge/subsoil. No pesticides or PCBs are reported in any of the soil samples. Based on these results and the historical use of the site, it is evident that there is no pesticide or PCB contamination of the soil adjacent to the LAOCB. No dioxins or furans are reported above method detection limits in the analyzed samples. Based on these results and the historical use of the site, it is evident that there is no dioxin or furan contamination of soil adjacent to the LAOCB.

\section{$\underline{\text { Soil Along the LAOCB Pipelines }}$}

Soil samples were collected to a maximum depth of approximately $3 \mathrm{~m}(10 \mathrm{ft})$ bls along the LAOCB pipeline to detect evidence of release from the pipeline that may have occurred during operation. A summary of the contaminants detected in the soil along the LAOCB pipeline is 
included as Table 1-5. The nature and extent of contamination in the soil along the LAOCB pipeline can be summarized as follows:

- Radionuclides species: Based on field screening results, it is evident that there is no manmade radionuclide contamination of soil along the LAOCB pipeline.

- Metals: Nineteen metals are reported in the soil samples adjacent to the LAOCB (Table 1-4). Review of the analytical data from soil adjacent to the LAOCB pipeline indicate that metal concentrations reported for samples collected along the LAOCB pipeline are consistent with those reported for the soil adjacent to the LAOCB (Table 1-5).

- Organic constituents: Acetone, carbon disulfide, methyl isobutyl ketone, and toluene are reported at concentrations above the screening levels in two to 12 percent of samples analyzed. However, all detections are also reported in the laboratory method blanks and were often estimated values. No apparent vertical or lateral trends in these data are evident that might indicate concentration gradients or hot spot areas.

- Methyl-ethyl-ketone is reported at concentrations above the screening values in 32 percent of the samples analyzed. The detections are for samples located in the vicinity of the two inferred leaks along the pipeline and in the vicinity of the upper end of the pipeline at the Hot Shop. The soil gas survey indicates that VOCs [primarily tetrachloroethene (PCE)] are reported in the vicinity of the LAOCB pipeline with probable origin near two areas along the pipeline. In addition, groundwater is also contaminated with PCE and trichloroethene (TCE), with probable origin near these two areas along the LAOCB pipeline. Chloromethane and chloroform (which may originate as breakdown products of PCE) are also present in the groundwater. The relative absence of PCE and TCE in the soil samples collected along the pipeline may be attributed to the fact that no decontamination fluids have flowed through the pipeline in approximately 15 
years and residual PCE/TCE originating from a break or leak in a pipeline has likely migrated vertically to the water table and degraded (e.g., biodegradation) to nondetectable concentrations in the soil. Despite the apparent absence of PCE and TCE in the soil samples, it is probable that some amounts of these compounds are present in nondetectable concentrations in an adsorbed phase in the vadose zone soil along the LAOCB pipeline. It must be noted that all detected VOCs are well below risk-based PRGs.

- No SVOCs are reported at concentrations exceeding screening values in soil samples from any locations along the LAOCB pipeline (Table 1-5).

- $\quad$ No pesticides or PCBs are reported for any of the soil samples. Based on this result and the historical use of the site, it is evident that there is no pesticide or PCB contamination at the site. Dioxins, furans, and total petroleum hydrocarbons (TPH) were not analyzed in the samples collected along the LAOCB pipeline.

\section{$\underline{\text { LAOCB Pipelines }}$}

The LAOCB pipeline was only sampled at each end. The interior surfaces of the pipeline are known to be radioactive as evidenced by measurable radioactivity [300,000 disintegrations per minute (dpm)] at both ends of the pipeline. Therefore, to minimize investigation derived waste, a video and radioactivity survey of the interior of the pipeline to identify cracks and/or obstructions was not performed.

\section{$\underline{\text { LAOCB Standing Water }}$}

A summary of the contaminants detected in the LAOCB standing water is included as Table 1-6. The nature and extent of contamination in the LAOCB standing water can be summarized as follows: 
- Radionuclide species: Seven radionuclides were reported in standing water samples collected from the LAOCB; bismuth-214 $\left({ }^{214} \mathrm{Bi}\right),{ }^{137} \mathrm{Cs},{ }^{60} \mathrm{Co}$, potassium- $40\left({ }^{40} \mathrm{~K}\right)$, and ${ }^{90} \mathrm{Sr}$ were detected at concentrations exceeding risk-based PRGs for residential water of these radionuclides. ${ }^{137} \mathrm{Cs},{ }^{60} \mathrm{Co}$, and ${ }^{90} \mathrm{Sr}$ are believed to originate from the LAOCB sludge/subsoil. The ${ }^{214} \mathrm{Bi}$, thallium-208 $\left({ }^{208} \mathrm{Tl}\right)$, and ${ }^{40} \mathrm{~K}$ detected in the standing water are probably of natural origin. Lastly, ${ }^{208} \mathrm{Tl}$ was reported at concentrations not exceeding the PRGs.

- Maximum ${ }^{3} \mathrm{H}$ activity in the Basin water was reported to be $8.6 \mathrm{pCi} / \mathrm{ml}$ at the time of sample collection. This activity is below the Maximum Contaminant Level (MCL) (20 $\mathrm{pCi} / \mathrm{ml}$ ) and is very low considering the ${ }^{3} \mathrm{H}$ activity in the sludge.

- Metals: Nine metals are reported in the standing water samples collected within the LAOCB. $\mathrm{Mn}$ is reported at concentrations exceeding the screening value (risk-based $\mathrm{PRG})$. No screening values are available for calcium $(\mathrm{Ca})$, iron $(\mathrm{Fe})$, or aluminum $(\mathrm{K})$. These metals likely originated from diffusion or desorption from the LAOCB sludge. Aluminum ( $\mathrm{Al}), \mathrm{Ba}, \mathrm{Cd}$, nickel $(\mathrm{Ni})$, and $\mathrm{Zn}$ are reported at concentrations below screening values.

- Organic constituents: Six VOCs are above method detection limits. These VOCs are 1,2-dichloropropane, benzene, bromomethane, carbon disulfide, dichloromethane, and styrene. When detected, these concentrations are estimated values below the quantitation limits and with poor precision. With the exception of bromomethane, all concentrations are well below the screening values (risk-based PRGs). Due to the very small difference between the screening value and the reported concentration for bromomethane and the poor precision of the reported concentration, an exceedance of the screening value cannot be determined with confidence. 
- Standing water samples collected were not analyzed for TPH. However, based on the results of the TPH analyses for the LAOCB sludge, it is expected that some petroleum hydrocarbons may be present in the standing water.

- No SVOC, pesticide, PCB, dioxin, or furan analyses were conducted on the standing water. Based on the disposal history of the LAOCB, no pesticides, PCBs, dioxins, or furans are expected within the standing water.

\section{Vegetation}

The nature and extent of contamination of vegetation in the LAOCB can be summarized as follows:

- $\quad$ Radionuclide species: ${ }^{137} \mathrm{Cs},{ }^{60} \mathrm{Co},{ }^{57} \mathrm{Co},{ }^{212} \mathrm{~Pb},{ }^{40} \mathrm{~K}$, ruthenium-106 $\left({ }^{106} \mathrm{Ru}\right)$, and ${ }^{234} \mathrm{Th}$ are reported in the vegetation samples collected within the LAOCB. Mean ${ }^{137} \mathrm{Cs}$ concentrations are significantly higher at the LAOCB than similar vegetation types at the reference area. The highest concentrations were detected in vegetation collected nearest the water (black willow, rush, and sedge). In addition, mean concentrations in vegetation are much higher than the mean concentration of ${ }^{137} \mathrm{Cs}$ in $\mathrm{SRS}$ soil $(0.15 \mathrm{pCi} / \mathrm{g})$ estimated by Fay and Pickett (1987). Mean ${ }^{60}$ Co concentrations in vegetation at the LAOCB appear to be significantly higher than the trace levels which normally occur in plants; however, it was not analyzed for in the samples from the reference area. ${ }^{60} \mathrm{Co}$ and ${ }^{137} \mathrm{Cs}$ concentrations detected at the LAOCB are likely to be unit-related and may be ecologically significant. ${ }^{57} \mathrm{Co},{ }^{212} \mathrm{~Pb},{ }^{40} \mathrm{~K},{ }^{106} \mathrm{Ru}$, and ${ }^{234} \mathrm{Th}$ were either detected at relatively low levels in single samples within the LAOCB, or detected concentrations were similar to or less than concentrations in the same types of vegetation from the 
reference area. Detected levels of these radionuclides are unlikely to be unit-related and/or ecologically significant.

- Metals: Sixteen metals are reported in the vegetation samples collected in the LAOCB. All detected metal concentrations from samples collected from the LAOCB are either at or below those observed in the reference area, within acceptable background ranges for the SRS, and/or ecologically insignificant.

- Organic constituents: Vegetation was not analyzed for organic constituents.

\subsubsection{Conclusions Regarding Nature and Extent of Contamination}

Source Area Sludge and Subsoil

Specific conclusions contained in the LAOCB RFI/RI Report for source area sludge and subsoil are presented below. Contaminants detected within the source area sludge and subsoil are summarized in Tables 1-2 and 1-3.

- Based on facility records and analytical data, it is concluded that LAOCB received wastewaters which contained radioactive substances and metals. Wastewater flowed into the Basin from a bermed concrete drainage pad located outside the Basin perimeter fence and from an underground pipeline originating at the maintenance Hot Shop, and then seeped into the sludge and subsoil.

- The sludge is highly contaminated with radionuclides. Radionuclides concentrations in the subsoil should reach background activity levels within two feet of the top of the sludge. 
- Several metals are present in the sludge/subsoil which exceed the screening values (Al, $\mathrm{Cr}, \mathrm{Fe}$, manganese (Mn), K, Ca, Ni, magnesium (Mg), $\mathrm{Pb}$, vanadium (V), Be, and $\mathrm{Cd}$ ).

- There is no significant organic contamination in the sludge and subsoil.

\section{Soil Adjacent to the LAOCB}

Specific conclusions contained in the LAOCB RFI/RI Report for soil adjacent to the LAOCB appear below. Contaminants detected within soil adjacent to the LAOCB are summarized in Tables 1-2 and 1-4.

- $\quad \mathrm{Cr}, \mathrm{V}, \mathrm{Al}$, arsenic (As), and Fe are the only metals that exceed screening values

- There is no significant man-made radionuclide or organic contamination in the soil.

\section{Soil Along the LAOCB Pipeline}

Specific conclusions contained in the LAOCB RFI/RI Report for soil along the LAOCB pipeline appear below. Contaminants detected within soil along the LAOCB pipeline are summarized in Tables 1-2 and 1-5.

- Metal concentrations in soil collected to a maximum depth of 10 feet were consistent with those reported for soil adjacent to the LAOCB.

- There is no man-made radionuclide contamination in the soil.

- All detections of VOCs were well below risk-based PRGs. No SVOCs were detected in the soil. 


\section{$\underline{\text { LAOCB Pipelines }}$}

To date, radioisotopes have not been detected in groundwater from the pipeline vicinity or soils surrounding the pipelines. This suggests that radioisotopes are not leaking from the pipelines. Radioactivity measurements taken within the pipelines suggest that radioisotopes are present. However, given that the pipelines have been inactive for approximately 40 years, the mobility of contaminants appears to be minimal. The pipelines are composed of cast iron which could degrade overtime and release associated radioisotopes to the surrounding soils.

\section{$\underline{\text { LAOCB Standing Water }}$}

Specific conclusions contained in the LAOCB RFI/RI Report for LAOCB standing water appear below. Contaminants detected within the standing water are summarized in Tables 1-2 and 1-6.

- $\quad$ Of the five radionuclides detected at concentrations above risk-based $\mathrm{PRGs},{ }^{137} \mathrm{Cs},{ }^{60} \mathrm{Co}$, and ${ }^{90} \mathrm{Sr}$ are believed to originate from the sludge/subsoil.

- Metals present in the standing water likely originate from the sludge.

- Five of the six VOCs detected were well below screening limits. An exceedance of the screening value cannot be determined for bromomethane.

\section{$\underline{\text { Vegetation }}$}

Specific conclusions contained in the LAOCB RFI/RI Report for vegetation within the LAOCB appear below. Contaminants detected within the vegetation are summarized in Table 1-2. 
- Elevated radionuclide concentrations in vegetation within the LAOCB, and especially close to the water, are likely to be unit-related and may be ecologically significant.

- Metals concentrations are either at or below reference area concentration, within acceptable SRS background ranges, and/or ecologically insignificant.

\subsubsection{Contaminant Fate and Transport}

Contaminants at the LAOCB may migrate in the environment following release from both the primary and the secondary sources, by any of several release or transport mechanisms, including:

- suspension and dispersal by the wind of particulate contaminants or contaminants adsorbed to surface soil particles (fugitive dust generation)

- direct volatilization of organic compounds from surface soil and sediment to air

- . uptake of soil or water contaminants by vegetation

- leaching of deep soil contaminants into groundwater

\subsubsection{Chemical Persistence}

The persistence of a residual contaminant in the subsurface environment is dependent on the properties of the chemical itself and on the characteristics of the subsurface environment. Inorganic elements are not capable of being destroyed by treatment processes but are capable of being transformed into different compounds or species. The exception to this is the radioactive decay process which chemically transforms a radioactive element into a completely different element by virtue of reducing the number of protons in the atom's nucleus. Radioactive decomposition occurs through the emission of radiation by a first order reaction. The rate of decomposition is directly proportional to the amount of undecayed material. For radioactive isotopes, it is customary to express the decomposition rates in terms of half-life, or the time 
required for the amount of the isotopes to decrease to half its initial value. Half-lives of radioactive isotopes detected at LAOCB are given in Table 1-7. Radioactive decay will be the primary removal mechanism for radioisotopes present in the Basin under current conditions.

Other physical/chemical processes that may influence the persistence (i.e., mobility) of inorganic compounds in soil in the environment include ion exchange, chelation, and oxidation/reduction reactions. These processes are very complex in the soil matrix since they are influenced by the cation/anion exchange capacity of the soil and other chemical/physical processes. With the exception of ${ }^{3} \mathrm{H}\left({ }^{3} \mathrm{H}\right.$ moves with water), LAOCB radioactive isotopes appear to be adsorbed to organic carbon in the Basin sludge and subsurface soil since other anthropogenic radionuclides have been detected in groundwater.

\subsubsection{Chemical Migration}

Based on the nature and extent of contamination at the site, the principal contaminant is ${ }^{3} \mathrm{H} .{ }^{3} \mathrm{H}$ is present at high activities in the sludge/substrate of the LAOCB, and in moderate amounts in the water table aquifer downgradient of the LAOCB. The fate and transport of ${ }^{3} \mathrm{H}$ present in the LAOCB is described below.

The source of ${ }^{3} \mathrm{H}$ at the site is the LAOCB sediment, where ${ }^{3} \mathrm{H}$ concentrations range from an observed median of $175 \mathrm{pCi} / \mathrm{g}$ to an observed maximum of $15,498 \mathrm{pCi} / \mathrm{g}$. The ${ }^{3} \mathrm{H}$ in the sediment would require from 22 to 101 years to decay to a threshold screening level of $50 \mathrm{pCi} / \mathrm{g}$. ${ }^{3} \mathrm{H}$ from the sediment has been transported from the Basin sediment to the upper and lower water table aquifers through ionic diffusion, advective and capillary transport, and/or migration down the annulus of monitoring wells LCO-1 and LCO-4. The time frame from which the maximum concentration of tritium decays to $50 \mathrm{pCi} / \mathrm{ml}$ is 24.6 years and the MCL of $20 \mathrm{pCi} / \mathrm{ml}$ is 42.3 years. During a decay period of 24.6 years, the ${ }^{3} \mathrm{H}$ plume could migrate from 45 to $94 \mathrm{~m}$ (147.6 to $307.5 \mathrm{ft})$. 
Radioactive isotopes are found in significant amounts only in the LAOCB sediment, where they are apparently fixed to the organic carbon in the sediment. The radioisotopes that are found in the groundwater are probably naturally occurring and are not associated with prior waste disposal activities at the site. Additional time series data on radionuclide concentrations in the groundwater are needed to confirm the origin of the groundwater nuclides.

Metals are commonly found in elevated concentrations in soil at the site. This condition appears to be the natural background condition for the site; however, analysis of additional soil samples collected in the vicinity of L-Area but distant from the disposal facilities at the site are needed in order to confirm this observation. The few metals that are found in the groundwater are probably naturally occurring; however, additional time series data on concentrations of metals in groundwater is needed to confirm this observation.

\subsubsection{Summary of the Risk Assessment}

As part of the LAOCB RFI/RI process, a BRA was prepared to evaluate the potential risk to human health and the environment from radioactive and chemical contaminants identified in investigations at the LAOCB. The following sections outline the results of the human health risk characterization and the ecological risk characterization. A complete discussion of the risk assessment methodology, receptor analysis, the risk characterizations, and uncertainty within the characterizations can be found in the BRA report (WSRC, 1996b).

Unit-specific data from the RFI/RI were used to identify and screen chemicals of potential concern (COPCs). Exposure point concentrations were calculated and used to estimate potential exposures and risks to humans and wildlife. Carcinogenic risks and hazard indices (HIs), based on a combination of exposure scenarios, location, and receptors, were calculated and then compared to EPA risk guidelines [i.e., 1E-04 to $1 \mathrm{E}-06$ carcinogenic risk, $\mathrm{HI}>1$, and Ecological 
Effects Quotient $(\mathrm{EEQ})>1$ ]. If the COPCs were determined to contribute significantly to risk, they were identified as COCs. The information from the BRA, along with other information evaluated as part of the CMS/FS process, support the development of remedial alternatives for the unit.

\subsubsection{Human Health Risk Assessment}

Cancer risk and noncarcinogenic hazards were estimated for COPCs based on EPA guidance. Human health COPCs at LAOCB are listed in Table 1-8. The cancer risk from exposure to contaminants is expressed as the increased probability of developing cancer over a 70-year lifetime. The potential for adverse noncarcinogenic health effects is expressed as an $\mathrm{HI}$, the sum of chemical-specific hazard quotients. Estimates of reasonable maximum exposure (RME) risk were developed for each potentially complete exposure pathway. The RME estimate is the highest exposure that is reasonably expected to occur in a small but definable "high-end" segment of the potentially exposed population. A summary of the future human health risk estimated for on-unit workers and residents exposed to the LAOCB contaminated media is depicted in Figures 1-7a through 1-7h.

Based on the current inactive industrial status of the Unit, the only current human receptor is the on-unit sampler (soil and/or groundwater). Estimates of total risk for this receptor by pathway for radioactive carcinogens resulted in risks above $1 \mathrm{E}-06$. This risk (3E-06) is due primarily to ${ }^{60} \mathrm{Co}$ and is a result of external exposure to gamma radiation from soil inside the LAOCB. Estimated risk for nonradioactive carcinogens are all less than the EPA point of departure risk level of $1 \mathrm{E}-06$, consequently carcinogenic risk for nonradionuclides is not a concern under the assumed current exposure scenario. In addition, none of the HIs for the current on-unit sampler (soil and/or groundwater) exceeded one, indicating that noncarcinogenic COPCs do not pose a threat of adverse human health effects. 
Under the hypothetical future industrial worker scenario, ingestion of soil from the LAOCB has a radiological risk of $2 \mathrm{E}-04$ due principally to ${ }^{241} \mathrm{Am},{ }^{90} \mathrm{Sr}$, and ${ }^{239} \mathrm{Pu}$, and exposure to external radiation directly from LAOCB soil has a risk of $2 \mathrm{E}-02$, with ${ }^{60} \mathrm{Co}$ and ${ }^{137} \mathrm{Cs}$ being the predominant contributors. The nonradioactive carcinogenic risks greater than the EPA target risk range and/or an $\mathrm{HI}$ greater than one were identified for the inhalation pathway within the LAOCB. The risk is due principally to $\mathrm{Cr}$. The carcinogenic and noncarcinogenic hazards for the hypothetical future industrial worker are summarized in Figure 1-7a.

Under the hypothetical future resident scenario, ingestion of soil from the LAOCB has a radiological risk of $6 \mathrm{E}-04$, due principally to ${ }^{241} \mathrm{Am},{ }^{90} \mathrm{Sr}$, and ${ }^{239} \mathrm{Pu}$, and exposure to external gamma radiation directly from $\mathrm{LAOCB}$ soil has a radiological risk of $2 \mathrm{E}-01$ due primarily to ${ }^{60} \mathrm{Co}$ and ${ }^{137} \mathrm{Cs}$. Estimated radiological risk levels for ingestion of fruit produce.(2E-03) leafy produce (9E-04), and tuberous produce (3E-03) were due to ${ }^{90} \mathrm{Sr}$ and ${ }^{137} \mathrm{Cs}$. The carcinogenic risk and noncarcinogenic hazards for the potential on-unit hypothetical resident exposure pathways are summarized in Figure 1-7e.

\subsubsection{Ecological Risk Assessment}

The purpose of the ecological risk assessment component of the BRA is to evaluate the likelihood that adverse ecological effects are occurring or may occur as a result of exposure of ecological components to contaminants associated with the LAOCB.

The specific methodology followed in this ecological risk assessment consists of a two-tiered evaluation. The first tier of the process is a Screening Evaluation, in which screening-level chemicals of potential concern (screening COPCs) are evaluated with regard to their potential to pose ecological risk based on consistently conservative assumptions. The methodology followed in the Screening Evaluation consists of three main elements: Unit Characterization, including preliminary problem formulation and preliminary evaluation of potential ecological effects 
associated with the screening COPCs identified at the unit; Preliminary Unit Calculation of exposure and risk estimates; and Preliminary Risk Characterization based on the results of the previous elements of the process. Any screening COPCs which fail the Screening Evaluation are termed COPCs and are carried into the second tier of the process, the Focused Evaluation.

The Focused Evaluation consists of essentially the same steps as the Screening Evaluation, but is based on more unit-specific and realistic assumptions. Accordingly, it provides a means of assessing whether COPCs identified as having a potential to pose ecological risk in a very conservative screening evaluation are actually likely to pose risk under existing or future conditions at the unit.

The Screening Evaluation resulted in the identification of nine metals $(\mathrm{Al}, \mathrm{Ba}, \mathrm{Cd}, \mathrm{Cr}, \mathrm{Cu}, \mathrm{Pb}$, $\mathrm{Mn}, \mathrm{Tl}$, and $\mathrm{V}$ ) that are considered COPCs because they have hazard quotients (HQs) exceeding a value of one. In addition to these metals, which are automatically classified as COPCs and included in the Focused Evaluation, several other screening COPCs are identified as having the potential to pose risk through bioaccumulation and/or biomagnification, event though they are below toxic levels in soil and their HQs are less than one. On this basis, octachlorodibenzo-p-dioxin isomers (OCDD) also is considered a COPC and carried through to the Focused Evaluation. An evaluation of risks from selected radionuclides in the LAOCB proper indicated no risk to ecological receptors.

In the Focused Evaluation, more realistic and unit-specific assumptions are used in reevaluating the COPCs identified in the Screening Evaluation. Intakes are calculated based on a range of area-use factors $(100,50,5$ percent $)$ and toxicity benchmarks are based on lowest-observedadverse-effect levels (LOAELs) for mortality or reproductive effects, when available. Thus, the HQs calculated in this evaluation are a more realistic indication of the potential for risk to assessment endpoints. The proximate assessment endpoints selected for the Focused Evaluation are reductions in populations of small mammals inhabiting the unit (represented by the cotton 
mouse) and of amphibians inhabiting the seepage basin (represented by the spring peeper frog). The ultimate assessment endpoint selected is a reduction in biodiversity of the ecological community encompassing the unit.

When based on 100 percent use of each of the areas being evaluated with the LAOCB, the COPCs identified as having a potential to pose ecological risk because they have an HQ greater than one are $\mathrm{Al}$ and $\mathrm{Cr}$.

When evaluated on the basis of the assumption of 50 percent usage of these areas by a receptor, only $\mathrm{Al}$ has an HQ exceeding one at any area. When five percent usage of these areas is assumed, there are no COPCs which exceed the HQ benchmark of one. The small extent of each of these areas within the OU and the limited habitat they provide make 100 percent use of any one area by an assessment endpoint unlikely. Because Cr exceeds the HQ benchmark of one by only a very small margin, any use of this area by less than 100 percent will essentially reduce these HQs to below the level of concern. Although the HQ for Al still exceeds a value of one when 50 percent use of the areas is assumed, it is generally very low in toxicity, particularly when it is complexed (as it is in the environment) and not in a free form (as it was in the toxicity test, which the toxicity benchmark value was based). Thus, the reduced bioavailability of these metals in the environment and the conservativeness of the toxicity benchmarks on which the HQs: are based is another indication that risk associated with these two metals is likely to be minimal. Therefore, they are not considered COCs.

The other COPC identified in the Focused Evaluation on the basis of the potential to bioaccumulate/biomagnify to toxic levels is OCDD. OCDD was identified in soil adjacent to the LAOCB.

OCDD is potentially toxic, lipophilic, and resistant to degradation. Based on these characteristics, OCDD is estimated to have a potential to pose risk to assessment endpoints. 
However, this compound is at such low levels that it must bioaccumulate significantly (from two to nine orders of magnitude) in order to approach toxic levels in mice. This would likely require a much longer period of time than the lifespan of a mouse or other assessment endpoint species. Also, wide ranging predators at higher trophic levels would not be sufficiently exposed to prey contaminated by this unit to be a risk. Therefore, OCDD is not considered to pose a risk to either proximate or ultimate assessment endpoints at the LAOCB. Therefore, there are no identified ecological COCs at the LAOCB.

\subsubsection{Risk Summary}

During the development of the BRA, it was determined that a majority of the risk to human health and the environment posed by this waste unit is associated with the radionuclides (representing greater than $99 \%$ of the risk) within the LAOCB. Specifically, the radionuclides in the sludge/subsoil in the LAOCB represent as much as $2 \mathrm{E}-01$ risk (future resident exposure to

radiation) with a majority of the risk due to gamma emitting radionuclides (e.g., ${ }^{137} \mathrm{Cs}$ and ${ }^{60} \mathrm{Co}$ ). In addition, during the development of the BRA, it was determined that the COPCs with risk values between $1 \mathrm{E}-04$ and $1 \mathrm{E}-06$ are radionuclides and metals, and represent less than $0.04 \%$ of the total unit risk. Therefore, since a majority of the risk is posed by radionuclides in the LAOCB and the COPCs that represent risk between 1E-04 and 1E-06 are radionuclides and metals with very similar physical and chemical properties, this CMS/FS was focused on the development of remedial alternatives for the COPCs that represent risks exceeding 1E-04. 
PHASE I FOCUSED CMS/FS REPORT

L-AREA OIL AND CHEMICAL BASIN
WSRC-RP-96-106, REV. 1.1

FEBRUARY 1997

003664

CHAPTER 1

FIGURES 
PHASE I FOCUSED CMS/FS REPORT

L-AREA OIL AND CHEMICAL BASIN
WSRC-RP-96-106, REV. 1.1

FEBRUARY 1997

THIS PAGE INTENTIONALLY LEFT BLANK

$1-38$

FINAL 

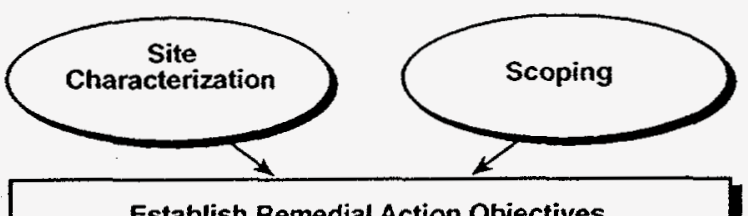

Establish Remedial Action Objectives

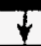

Develop General Response

Actions Describing Areas or

Volumes of Media to Which

Containment, Treatment, or

Removal Actions May Be Applied

\section{1}

Identify Potential

Treatment and

Disposal Technologies

and Screen Based on

Techrical Implementability

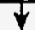

Evaluate Process Options Based

on Effectiveness, Implementability,

and Relative Cost, to Select a

Representative Process for each

Technology Type

Repeat Previous Scoping Steps:

-Determine New Data Needs

-Develop Sampling Strategies

and Analytical Support to

Aquire Additional Data

-Repeat Steps in RI Site

Characterization
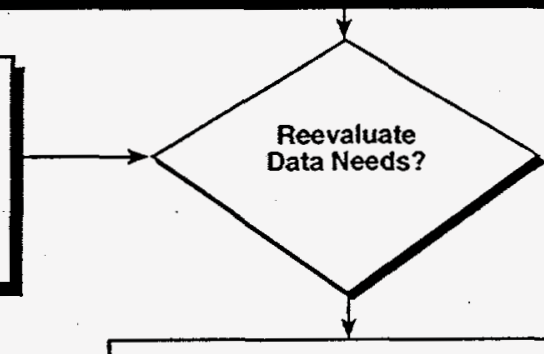

Combine Media-Specific

Technologies into Alternatives

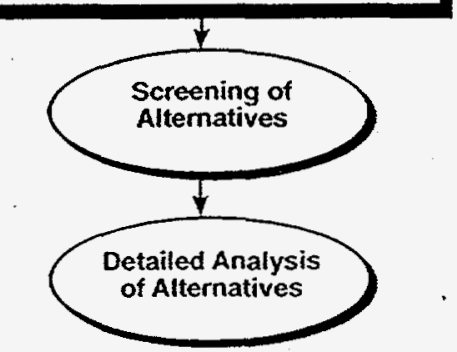

Source: EPA, 1988a

Figure 1-1 Overall Feasibility Study/Corrective Measures Study Process 
PHASE I FOCUSED CMS/FS REPORT

L-AREA OIL AND CHEMICAL BASIN
WSRC-RP-96-106, REV. 1.1

FEBRUARY 1997

THIS PAGE INTENTIONALLY LEFT BLANK 


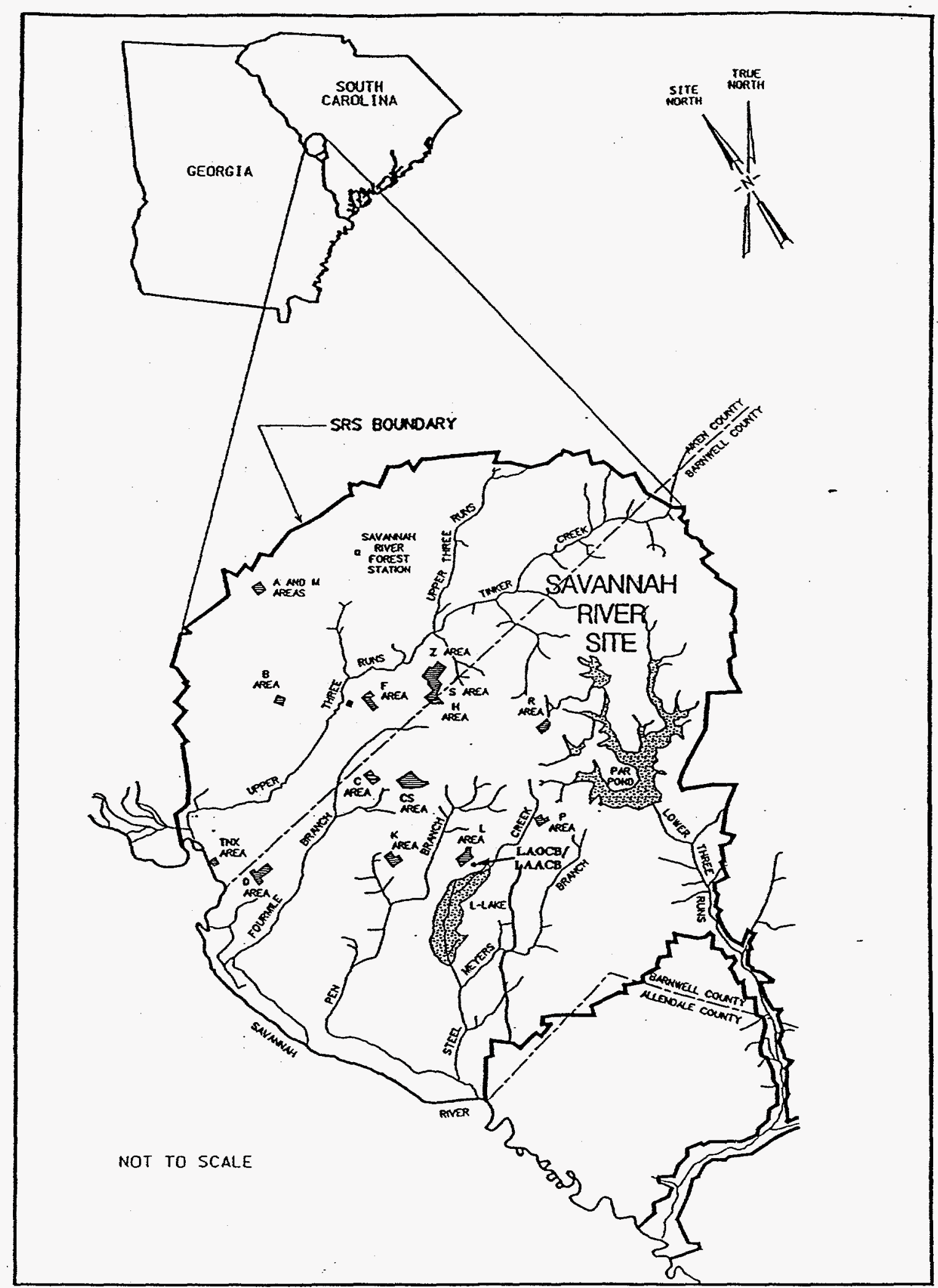

Figure 1-2 Location of the Savannah River Site (SRS) and Major SRS Facilities 


\section{THIS PAGE INTENTIONALLY LEFT BLANK}




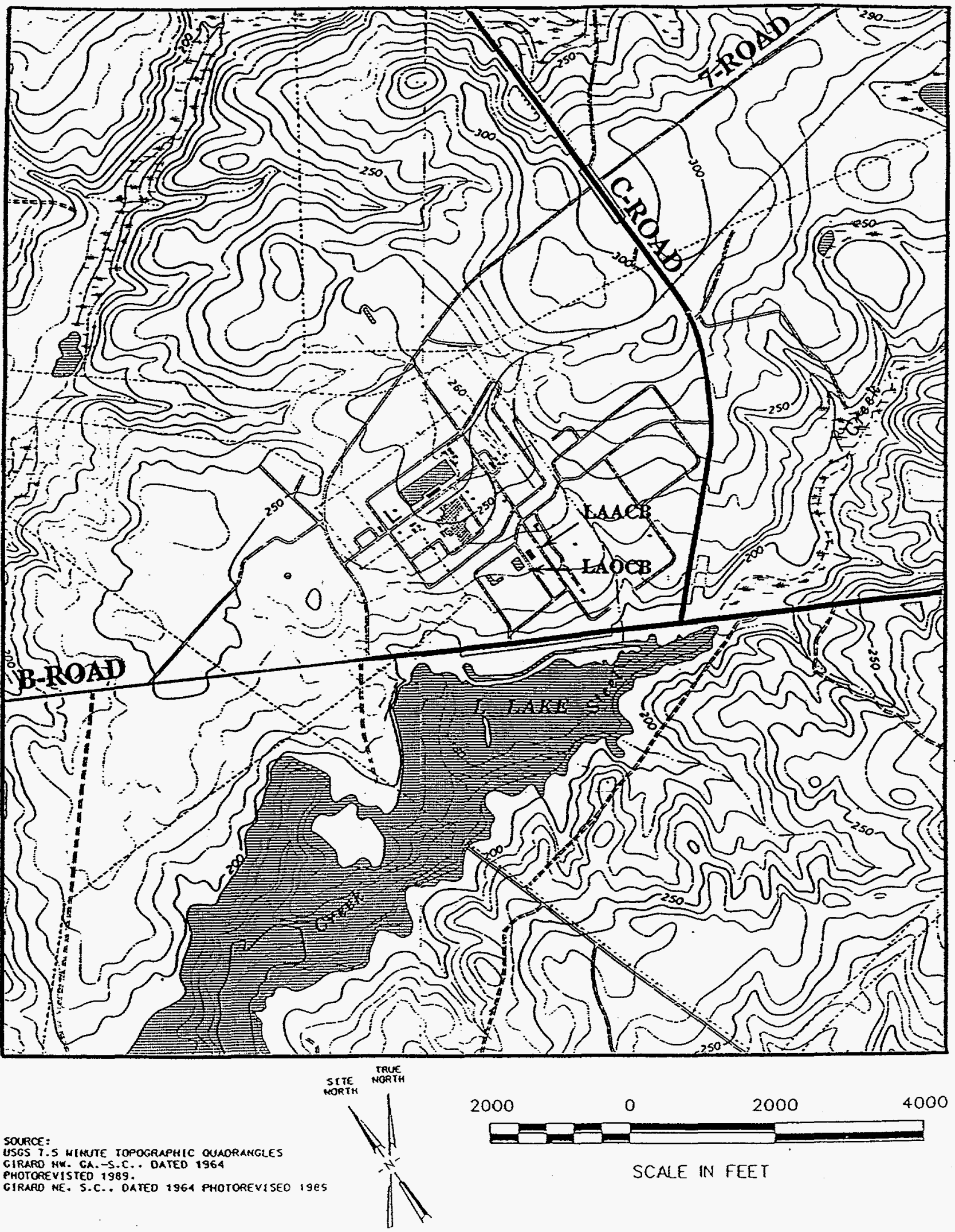

Figure 1-3 Topographic Map of the L-Area Oil and Chemical Basin and Surrounding Area 
PHASE I FOCUSED CMS/FS REPORT

L-AREA OIL AND CHEMICAL BASIN
WSRC-RP-96-106, REV. 1.1

FEBRUARY 1997

THIS PAGE INTENTIONALLY LEFT BLANK

$1-44$

FINAL 

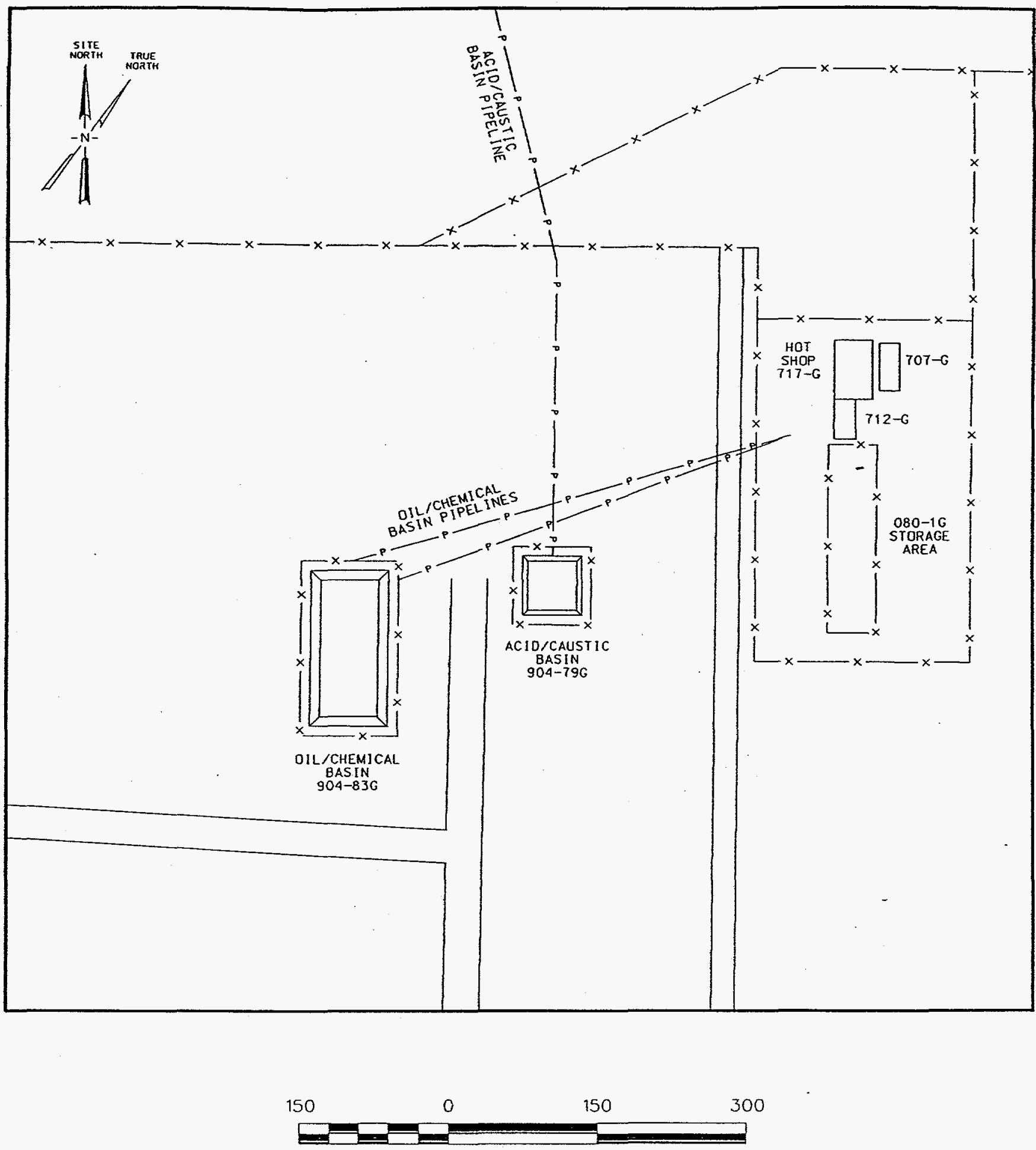

SCALE IN FEET

Figure 1-4 L-Area Oil and Chemical Basin and Vicinity 
Log Gross Alpha (pCi/g)

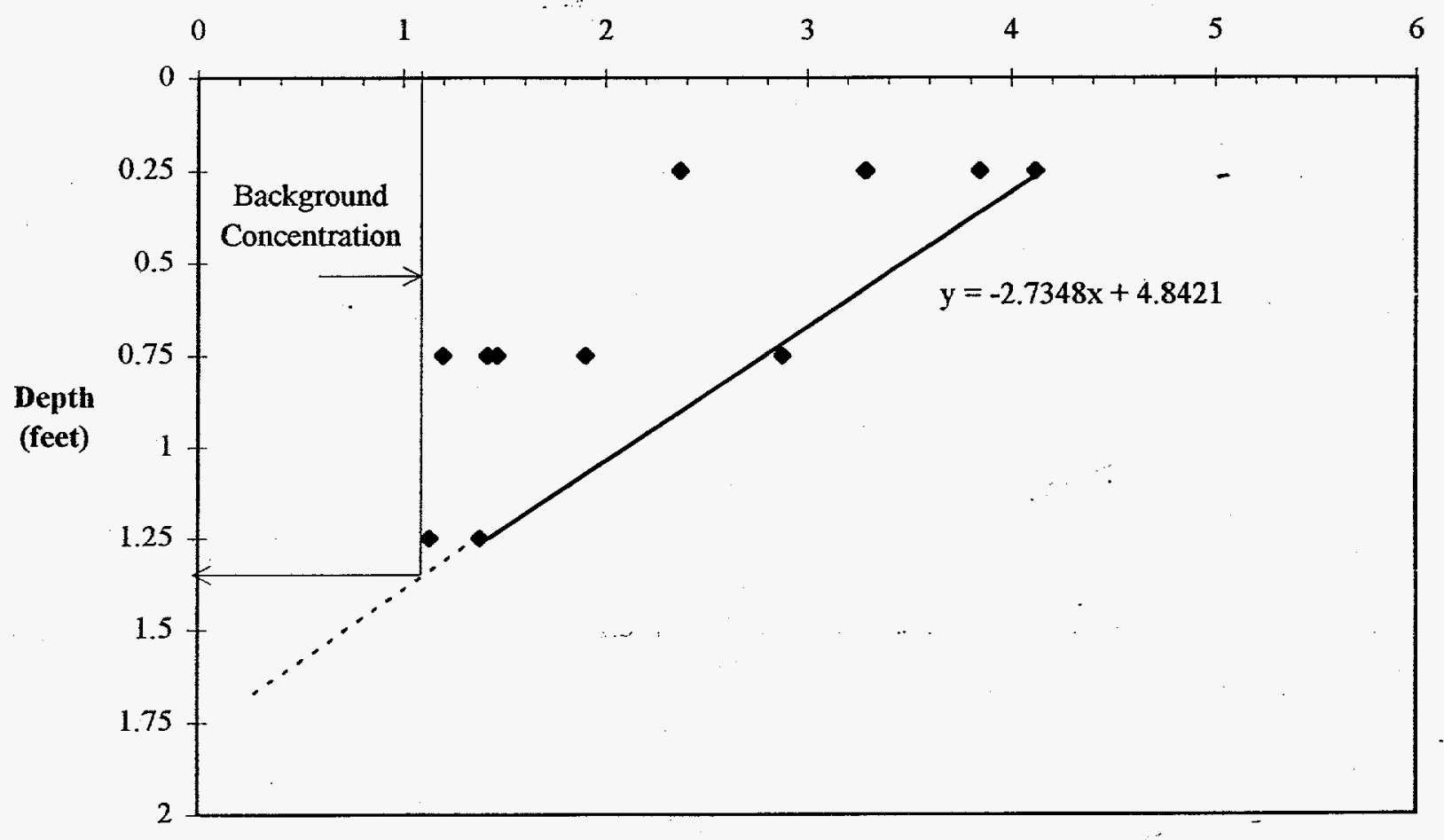

Figure 1-5 Gross Alpha Concentration Versus Depth 
PHASE I FOCUSED CMS/FS REPORT

L-AREA OIL AND CHEMICAL BASIN
WSRC-RP-96-106, REV. 1.1

FEBRUARY 1997

THIS PAGE INTENTIONALLY LEFT BLANK 
Log Gross Beta (pCi/g)

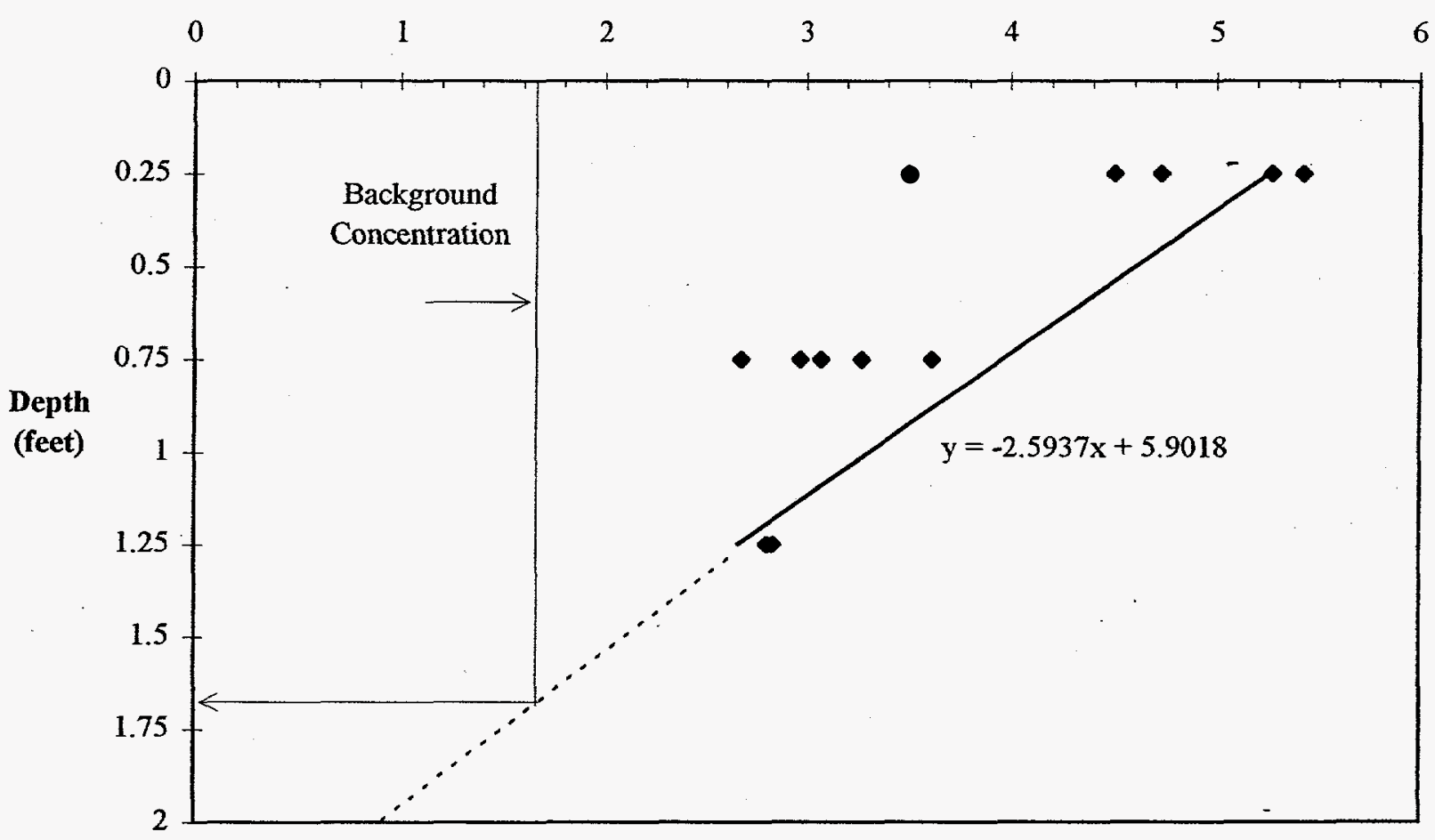

Figure 1-6 Gross Beta Concentration Versus Depth 
THIS PAGE INTENTIONALLY LEFT BLANK 


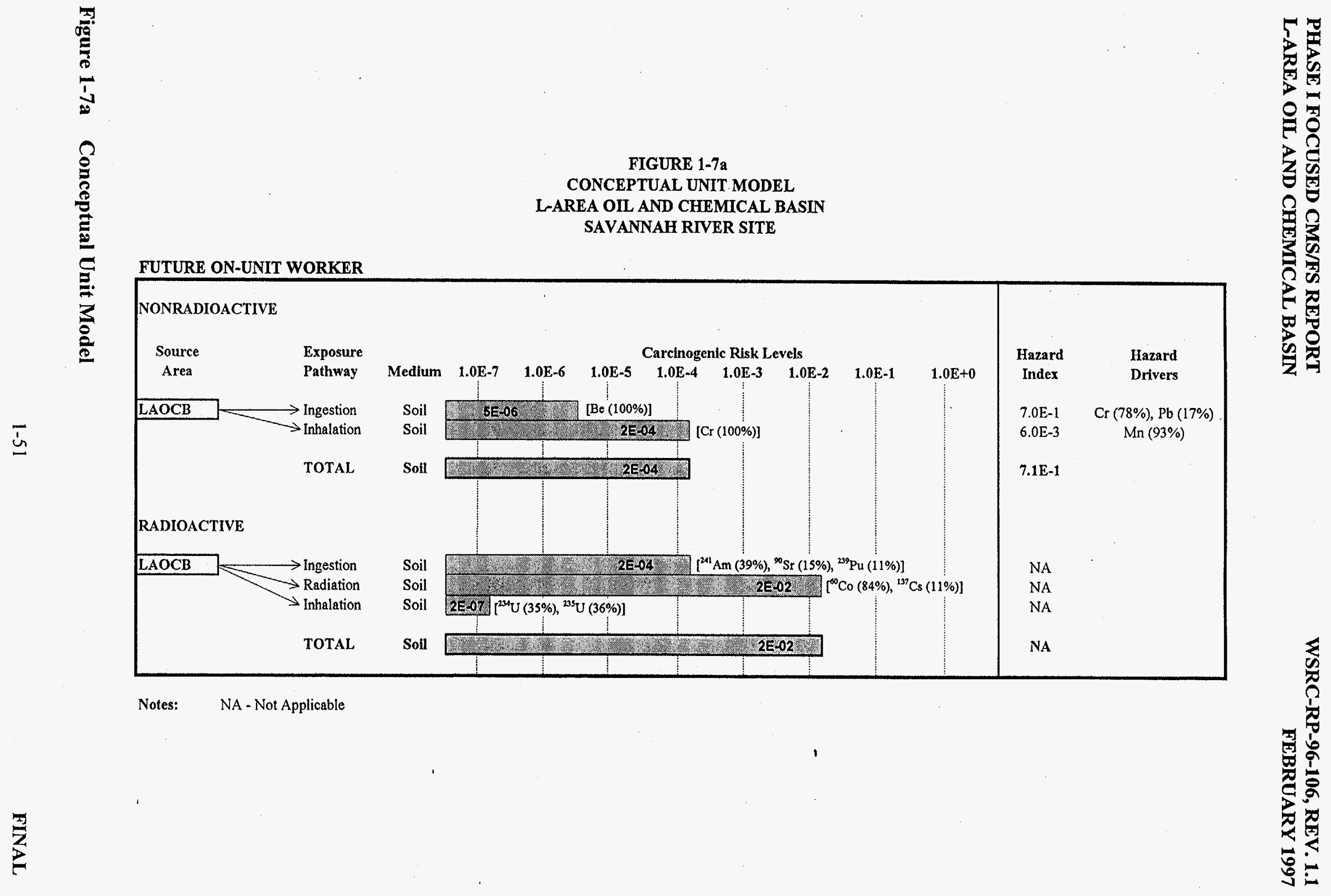




\section{THIS PAGE INTENTIONALLY LEFT BLANK}




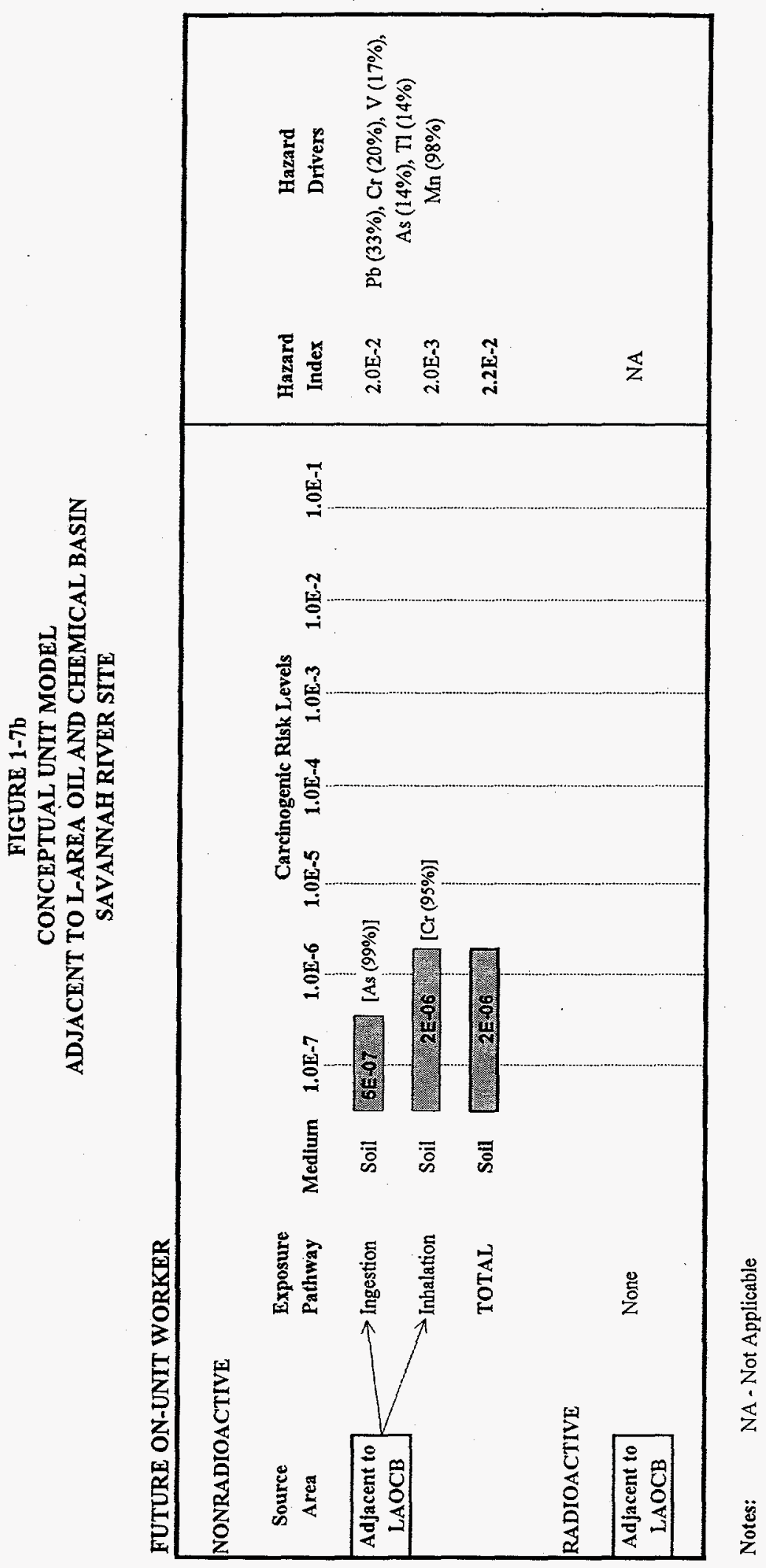

Figure 1-7b Conceptual Unit Model 
THIS PAGE INTENTIONALLY LEFT BLANK 


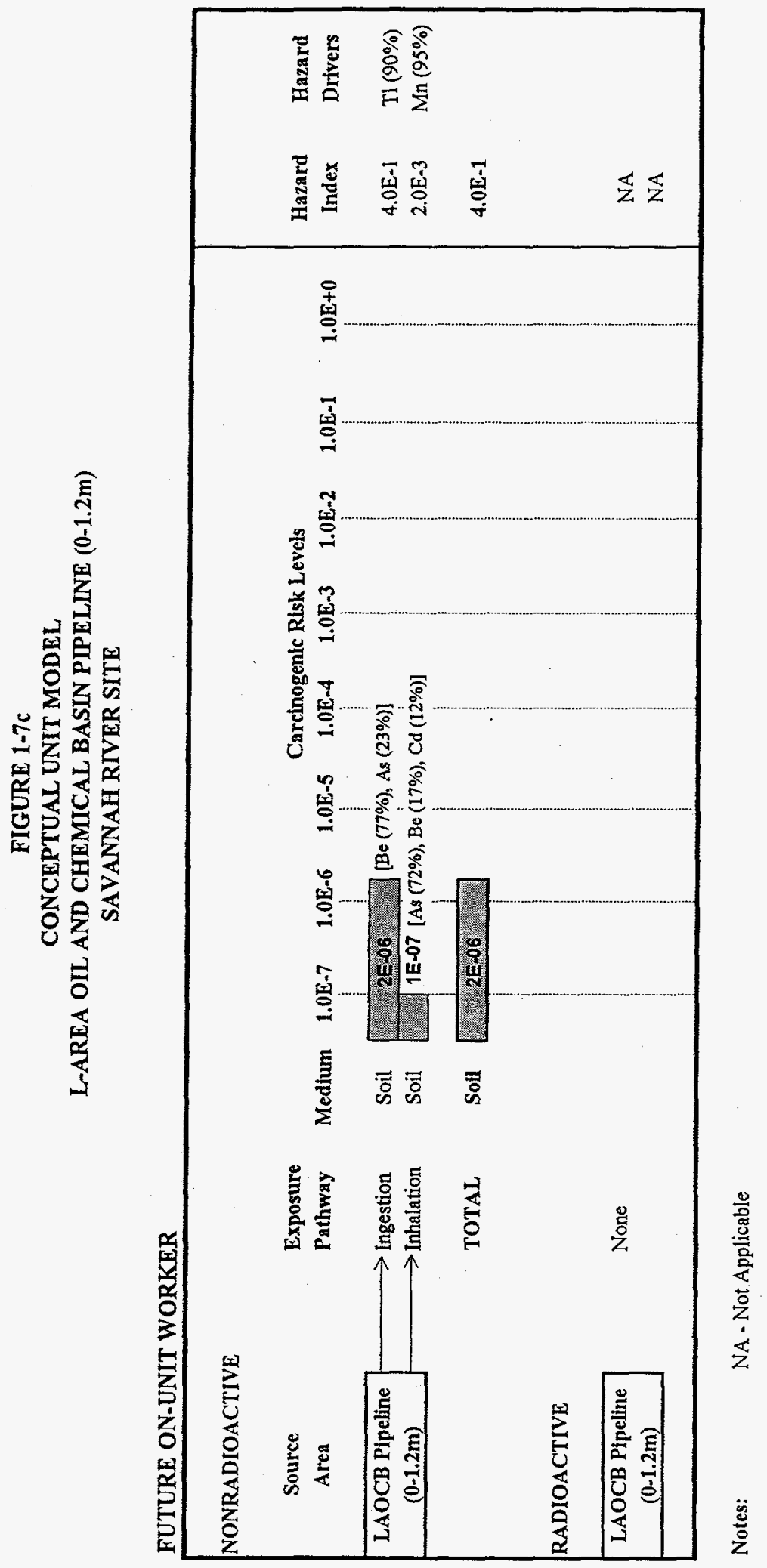

Figure 1-7c Conceptual Unit Model 


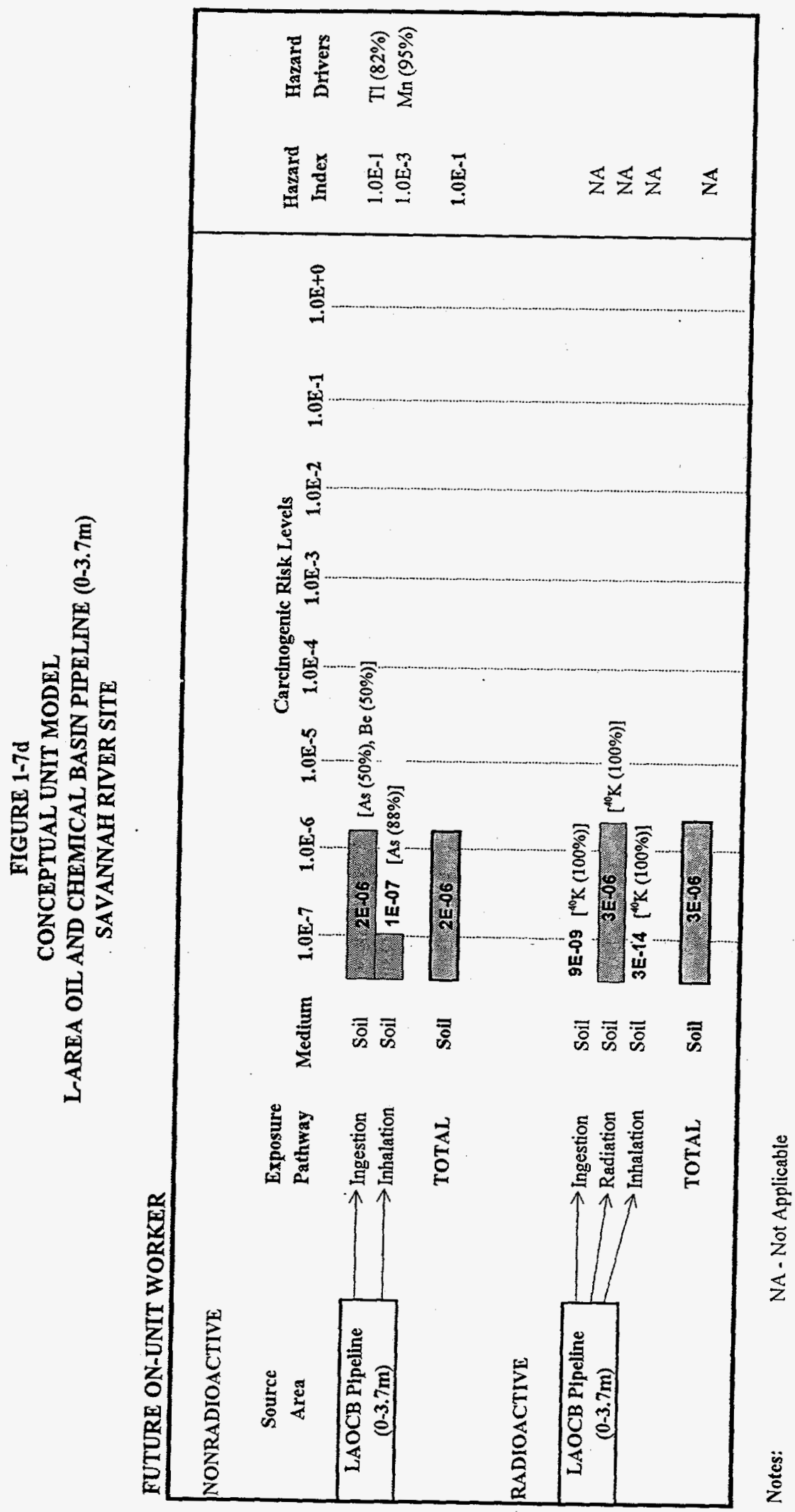

Figure 1-7d Conceptual Unit Model 
THIS PAGE INTENTIONALLY LEFT BLANK 


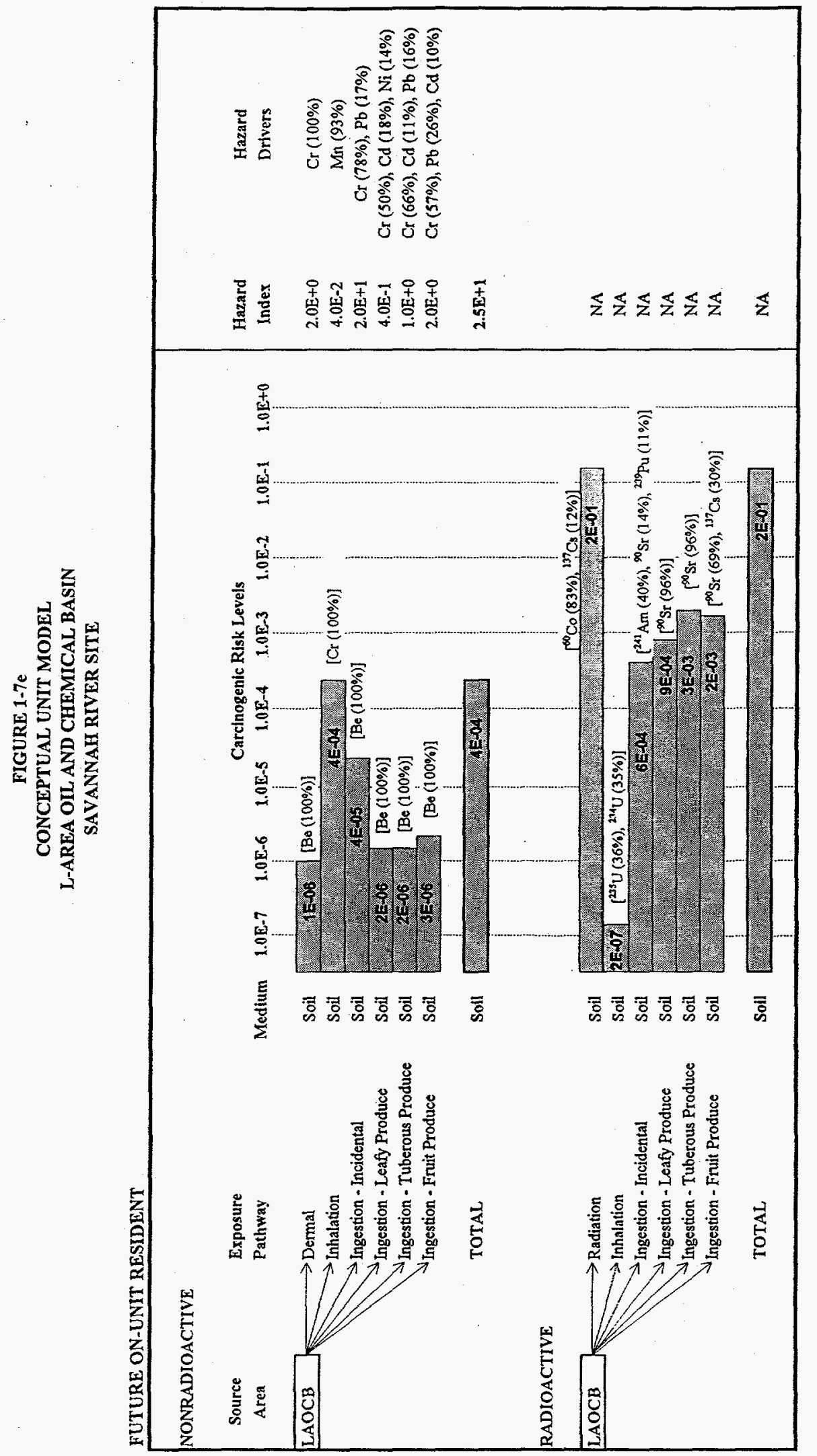

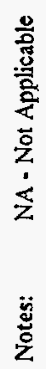

Figure 1-7e Conceptual Unit Model 
THIS PAGE INTENTIONALLY LEFT BLANK 


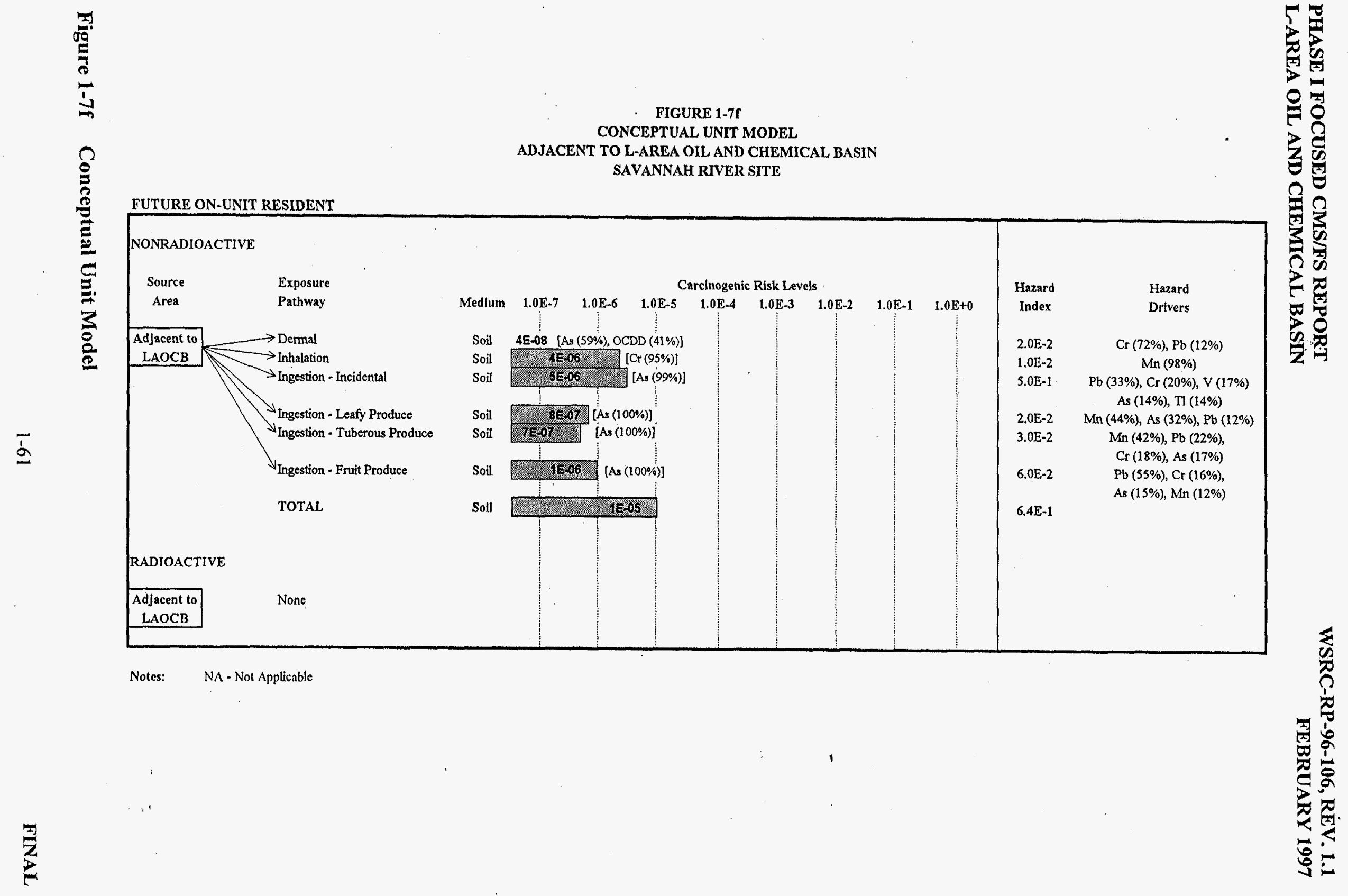




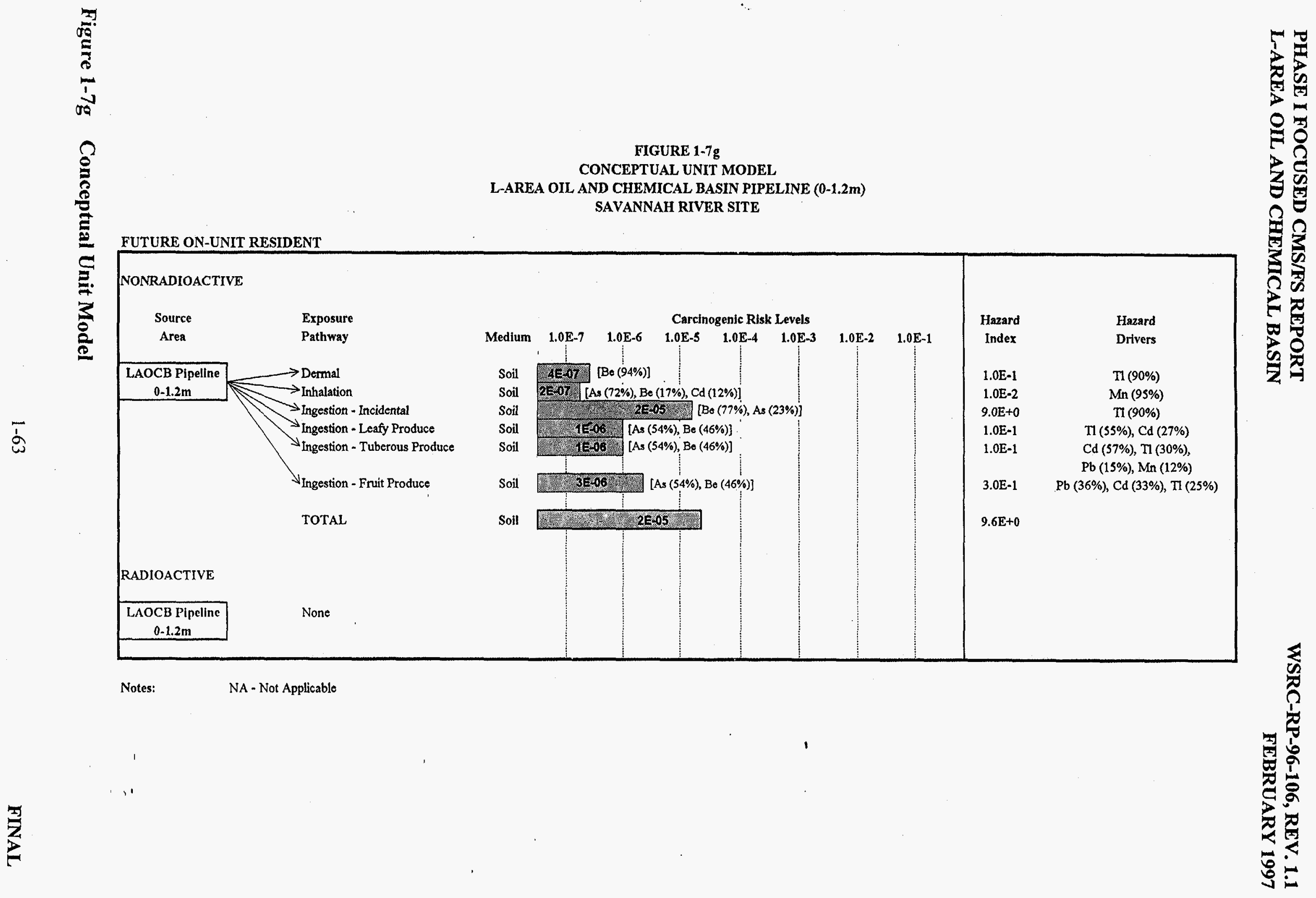




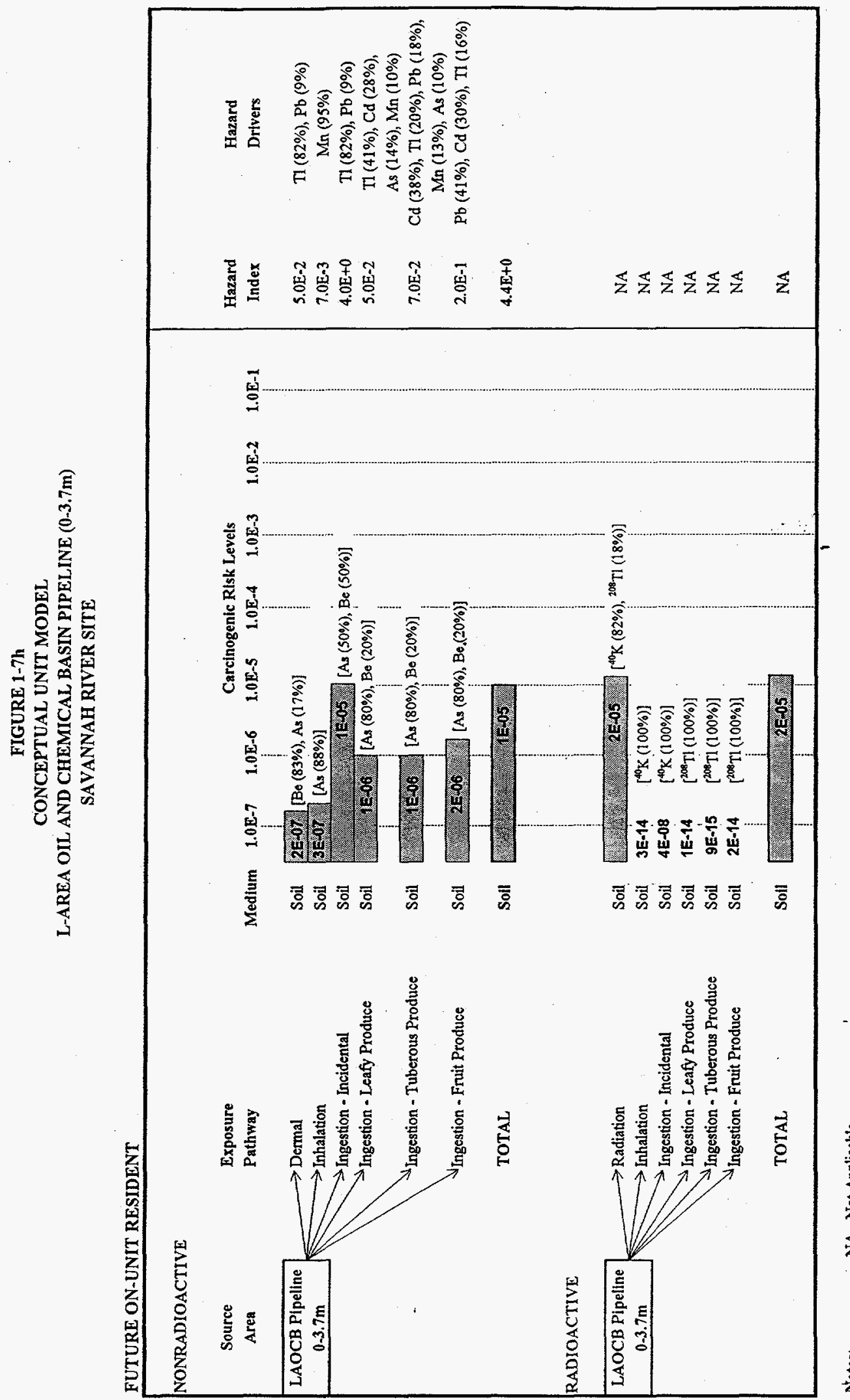

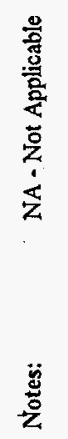

Figure 1-7h Conceptual Unit Model 
THIS PAGE INTENTIONALLY LEFT BLANK 
PHASE I FOCUSED CMS/FS REPORT

L-AREA OIL AND CHEMICAL BASIN
WSRC-RP-96-106, REV. 1.1

FEBRUARY 1997

CHAPTER 1

TABLES

FINAL 
THIS PAGE INTENTIONALLY LEFT BLANK 
TABLE 1-1

\section{PREFERRED HABITAT OF THREATENED AND ENDANGERED SPECIES \\ IN VICINITY OF L-AREA \\ L-AREA OIL AND CHEMICAL BASIN \\ SAVANNAH RIVER SITE}

\author{
Common Name \\ Scientific Name

\section{MAMMALS} \\ Southeastern Big-eared Bat \\ Plecotus rafenesquii

\section{BIRDS} \\ Bald Eagle \\ Haliaeetus leucocephalus \\ Loggerhead Shrike \\ Lanius ludovicianus \\ Bachman's Sparrow \\ Aimophila aestivalis \\ Osprey \\ Pandion haliaetus
}

\section{AMPHIBIANS}

Carolina Gopher Frog

Rana areolata capito

\section{REPTILES}

American Alligator Alligator mississippiensis

Florida Pine Snake

Pituophis melanoleucus

mugitus

Northern Pine Snake Pituophis melanoleucus melanoleucus

Southern Hognose Snake Heterodon simus

\author{
Federal \\ Status \\ State Heritage \\ Program Status
}

$\mathrm{C} 2$

G4S3

E

C2

C2

C2

$\mathrm{T}(\mathrm{SA})$

C2

$\mathrm{C} 2$

$\mathrm{C} 2$

E

G454

G3

SC

\author{
Preferred Habitat \\ (Potential to Occur)
}

Cave-type structures (may occur in nearby L-Area buildings/feed near basins)

Large reservoirs, Carolina bays (feed regularly in L Lake)

Large grass-forb/shrub opening (likely habitat around L Area)

Open fire-maintained longleaf pine/grass-forb/shrub openings (potential occur edges L Area)

Large reservoirs/standing snag (L Lake known feeding area)

Pine sandhills with underground burrows, reproduce shallow wetlands (potential occur in area)

Variety aquatic/wetland habitats (remotely possible occur in basins)

Pine sandhill areas (could occur in pine stands adjacent to basins)

Pine sandhill areas (could occur in pine stands adjacent in basins)

Pine sandhill areas (could occur in pine stands adjacent to basins) 
TABLE 1-1 (continued)

PREFERRED HABITAT OF THREATENED AND ENDANGERED SPECIES

IN VICINITY OF L-AREA

L-AREA OIL AND CHEMICAL BASIN

SAVANNAH RIVER SITE

Common Name

Scientific Name

PLANTS

Smooth Purple Coneflower Echniaacea laevigata

Wild Indigo

Baptisia lanceolata

Pink Tickseed

Coreopsis rosea

Epiphytic Sedge

Carex decomposita

Elliott's Croton

Croton elliottii

Little Bur-head

Echinodorus tenellus var.

parvulus

Swamp Lobelia

Lobelia boykinii

Spatulate Seedbox Ludwigia spathulata

Nestronis

Nestronia umbellula

American Nailwort

Paronychia americana

Green-fringed Orchid

Platanthera lacera

Awned Meadow Beauty

Rhexia aristosa
Federal

Status

E

G2S1

G2G3

$\mathrm{RC} / \mathrm{G} 3$

G3G4

G2G3

SL/G3

C2

G2

G2G3

$\mathrm{C} 2$

G2G4

G3

SL

C2

G2
Preferred Habitat

(Potential to Occur)

Prairie remnants-calcareous soil

Xeric sandhills

Carolina bays, herbaceous meadows

Swamp forest

Carolina bays, herbaceous meadows

Carolina bays, ditches, shallow depressions

Carolina bays, herbaceous

Carolina bays, ditches, shaHow depressions

Upland mixed pine-hardwood or bayheads

Xeric sandhills

Bottomland hardwood, Carolina bays

Carolina bays, herbaceous meadows 
TABLE 1-1 (continued)

PREFERRED HABITAT OF THREATENED AND ENDANGERED SPECIES

IN VICINITY OF L-AREA

L-AREA OIL AND CHEMICAL BASIN

SAVANNAH RIVER SITE

\author{
Common Name \\ Scientific Name \\ Drowned Hornedrush
Rhynchospora inundata \\ Stalkless Yellowcress \\ Rorippa sessiliflora \\ Slender Arrow-head \\ Sagittaria isoetiformes \\ Florda Bladderwort \\ Utricularia floridana \\ Dwarf Bladderwort \\ Utricularia olivacea
}

Federal

State Heritage

Preferred Habitat

Status

Program Status

(Potential to Occur)

G3G4

Carolina bays, ponds

G3G5 Wet open areas

G3G5

Carolina bays, ponds

G3G5

Carolina bays, ponds

SL

Ponds

\title{
LEGEND
}

E-Endangered; TSA-Threatened due to Similarity of Appearance; C2-Category 2; SL-State Listed; SC-State Concern; G1, G2, G3, G4, G5 - Globally Rare, listed in decreasing importance; S1, S2, S3, S4, S5 - Statewide Rare, listed in decreasing importance.

Source: WSRC, 1996b 
TABLE 1-2

SUMMARY OF DETECTED CONSTITUENTS

L-AREA OIL AND CHEMICAL BASIN

SAVANNAH RIVER SITE

\begin{tabular}{|c|c|c|c|}
\hline \multirow{2}{*}{ MEDIA } & \multicolumn{3}{|c|}{ DETECTED CONSTITUENTS } \\
\hline & Radionuclide Species & Metals & Organic Constituents \\
\hline Source Area Sludge and Subsoil & $\begin{array}{l}{ }^{241} \mathrm{Am},{ }^{137} \mathrm{Cs},{ }^{60} \mathrm{Co},{ }^{152} \mathrm{Eu},{ }^{154} \mathrm{Eu}, \\
{ }^{238} \mathrm{Pu},{ }^{239} \mathrm{Pu},{ }^{90} \mathrm{Sr},{ }^{234} \mathrm{U},{ }^{235} \mathrm{U},{ }^{238} \mathrm{U}, \\
{ }^{234} \mathrm{Th},{ }^{3} \mathrm{H}\end{array}$ & $\begin{array}{l}\mathrm{Al}, \mathrm{Cr}, \mathrm{Fe}, \mathrm{Mn}, \mathrm{K}, \mathrm{Ca}, \mathrm{Ni}, \mathrm{Mg}, \mathrm{Cu}, \mathrm{Pb} \\
\mathrm{V}, \mathrm{Be}, \mathrm{Cd}, \mathrm{Zn}, \mathrm{Ba}, \mathrm{Co}, \mathrm{Hg}\end{array}$ & $\begin{array}{l}\text { Petroleum hydrocarbons, methyl } \\
\text { ethyl ketone, ethylbenzene, carbon } \\
\text { disulfide, and toluene }\end{array}$ \\
\hline Soils Along LAOCB Pipeline & None & $\begin{array}{l}\mathrm{Al}, \mathrm{As}, \mathrm{Ba}, \mathrm{Be}, \mathrm{Cd}, \mathrm{Ca}, \mathrm{Cr}, \mathrm{Co}, \mathrm{Cu}, \mathrm{Fe} \\
\mathrm{Pb}, \mathrm{Mn}, \mathrm{V}, \mathrm{Mg}, \mathrm{K}, \mathrm{Ag}, \mathrm{Th}, \mathrm{Zn}\end{array}$ & $\begin{array}{l}\text { Acetone, carbon disulfide, methyl } \\
\text { ethyl ketone, methyl isobutyl } \\
\text { ketone, toluene }\end{array}$ \\
\hline Vegetation & ${ }^{137} \mathrm{Cs},{ }^{60} \mathrm{Co}$ & None & Not Analyzed \\
\hline
\end{tabular}


TABLE 1-3

LAOCB SLUDGE AND SUBSOIL

SUMMARY OF DETECTED CONSTITUENTS IN SOIL

\begin{tabular}{|c|c|c|c|c|}
\hline Analyte & $\begin{array}{c}\text { USEPA } \\
\text { Region } 3 \\
\text { PRGs } \\
\end{array}$ & $\begin{array}{c}\text { X Average } \\
\text { Background } \\
\text { Value }\end{array}$ & $\begin{array}{l}\text { Frequency of Detects } \\
\text { Above } 2 \text { X Average } \\
\text { Background }\end{array}$ & $\begin{array}{c}\text { Highest } \\
\text { Detection }\end{array}$ \\
\hline \multicolumn{5}{|c|}{ RADIOISOTOPES (pCi/g) } \\
\hline Actinium-228 & 0.0144 & $* 1.25$ & $9 / 12$ & 11.86 \\
\hline Americium-24l & 2.33 & $* 1.25$ & $9 / 12$ & 804.65 \\
\hline Antimony-125 & 0.0347 & $\% 0.34$ & $12 / 12$ & 7.44 \\
\hline Bismuth-214 & 0.00786 & $* 0.91$ & $9 / 12$ & 9.30 \\
\hline Cerium-144 & 1.64 & $* 0.91$ & $8 / 12$ & 6.98 \\
\hline Cesium-134 & 0.00801 & $* 0.11$ & $12 / 12$ & 2.09 \\
\hline Cesium-137 & 27.5 & $* 0.11$ & $12 / 12$ & 1154.20 \\
\hline Cobalt- 60 & 0.00484 & 0.11 & $12 / 12$ & 5241.80 \\
\hline Curium-244 & 4.79 & - & $+12 / 12$ & 339.72 \\
\hline Europium-154 & 0.0102 & $* 0.34$ & $12 / 12$ & 109.30 \\
\hline Europium-155 & 0.0706 & * 0.57 & $8 / 12$ & 4.88 \\
\hline Lead-212 & 0.149 & - & $+12 / 12$ & 2.79 \\
\hline Lead-214 & 0.0651 & $* 0.84$ & $6 / 12$ & 4.75 \\
\hline Plutonium-238 & 3.49 & - & $+10 / 10$ & 60.15 \\
\hline Plutonium-239 & 3.34 & - & $+10 / 10$ & 236.51 \\
\hline Potassium-40 & 0.0771 & 1.59 & $6 / 12$ & 15.81 \\
\hline Promethium-147 & 1830 & - & $+9 / 9$ & 93.98 \\
\hline Strontium-90 & 21.4 & $\ldots$ & $+[1 / 11$ & 2706.60 \\
\hline Thallium-208 & 0.0321 & $* 1.14$ & $5 / 12$ & 18.60 \\
\hline Tritium & $\ldots$ & $\overline{-}$ & $+11 / 11$ & 15498.27 \\
\hline Thorium-234 & 11.2 & - & $+12 / 12$ & 713.56 \\
\hline Uranium-234 & 46.5 & $\ldots$ & $+10 / 10$ & 2019.90 \\
\hline Uranium-235 & 0.173 & - & $+12 / 12$ & 44.07 \\
\hline Uranium-238 & 46.9 & - & $+12 / 12$ & 2203.30 \\
\hline Gross Alpha & - & 220 & $10 / 12$ & 13098.60 \\
\hline Non-Volatile Beta & $=$ & 250 & $12 / 12$ & 22625.90 \\
\hline \multicolumn{5}{|l|}{ VOCs $\mathrm{\mu g} / \mathrm{kg}$} \\
\hline Acetone & 780 & 19.34 & $5 / 12$ & 770.60 \\
\hline Carbon disulfide & 380 & 0.47 & $3 / 12$ & 15.90 \\
\hline Methyl ethyl ketone & 4700 & 49.38 & $2 / 12$ & 152.70 \\
\hline Toluene & 1600 & 3.24 & $2 / 12$ & 21.20 \\
\hline \multicolumn{5}{|l|}{ SVOCs $\mu \mathrm{g} / \mathrm{kg}$} \\
\hline \multicolumn{5}{|c|}{ No values observed above the $2 X$ average background values } \\
\hline \multicolumn{5}{|c|}{ PESTICIDES \& PCBs $\mu g / \mathrm{kg}$} \\
\hline \multicolumn{5}{|c|}{ Not Analyzed } \\
\hline \multicolumn{5}{|c|}{ DIOXINS, FURANS $\mu q k g$} \\
\hline \multicolumn{5}{|l|}{ Not Analyzed } \\
\hline \multicolumn{3}{|c|}{ TOTAL PETROLEUM HYDROCARBONS rag/kg } & & $\therefore$ \\
\hline TPH & - & $=$ & $9 / 12$ & 7185.60 \\
\hline \multicolumn{5}{|l|}{ METALS mg/kg } \\
\hline Aluminum & 23000 & 6124 & $12 / 12$ & 57681.00 \\
\hline Barium & 550 & 18.72 & $3 / 12$ & 77.45 \\
\hline Beryllium & 0.15 & 0.34 & $2 / 12$ & 15.83 \\
\hline Cadmium & 3.9 & 0.56 & $2 / 12$ & 12.33 \\
\hline Calcium & 820 & 749.2 & $7 / 12$ & 9697.00 \\
\hline Chromium & 39 & 10.72 & $12 / 12$ & 5250.00 \\
\hline Cobaht & 4700 & 3.68 & $3 / 12$ & 38.34 \\
\hline Copper & 290 & 3 & $4 / 12$ & 128.25 \\
\hline Iron & 24000 & 16923.8 & $8 / 12$ & 53461.00 \\
\hline Lead & 5 & 5.65 & $5 / 12$ & 242.94 \\
\hline Magnesium & 520 & 748.8 & $1 / 12$ & 793.32 \\
\hline Manganese & 39 & 22.2 & $12 / 12$ & 603.23 \\
\hline Mercury & 2.3 & 1.2 & $1 / 12$ & 1.56 \\
\hline Nickel & 160 & 3.06 & $4 / 12$ & 394.70 \\
\hline Potassium & 940 & 748.8 & $5 / 12$ & 2528.20 \\
\hline Vanadium & 55 & 38.08 & $5 / 12$ & 135.95 \\
\hline Zinc & 2300 & 4.4 & $12 / 12$ & 1877.04 \\
\hline
\end{tabular}

Legend:

Frequency: $5 / 12=$ Detects above $2 X$ average background values/total number of samples analyzed

* Background value is established from an atternate background soil boring (LAOCB-20). Samples collected from the designated background boring (LCO-8DL) were not speciated beyond Gross Alpha and Non-Volatile Beta.

+ No site-specific background value exists for anaiyte. Frequency reported is detectshotal number of samples analyzed. --- No value available.

PRGs - Risk-based (1E-06) Preliminary Remedial Goals

2 The screening levels of 20 and $50 \mathrm{pCi} / \mathrm{g}$ for gross alpha and non-volatile beta, respectively, are based on site-specific background samples and the presence of naturally occurring radionuclides (see Section 2.6.3).

Chemical Class headings labeled as "Not Analyzed" means that none of the constituents in the chemical class were analyzed. 
TABLE 1-4

SOIL ADJACENT TO THE LAOCB

SUMMARY OF DETECTED CONSTITUENTS IN SOIL

\begin{tabular}{|c|c|c|c|c|}
\hline Analyte & $\begin{array}{l}\text { USEPA } \\
\text { Region } 3 \\
\text { PRGs }\end{array}$ & $\begin{array}{l}2 \times \text { Average } \\
\text { Background } \\
\text { Value }\end{array}$ & $\begin{array}{c}\text { Frequency of Detects } \\
\text { Above } 2 \text { X Average } \\
\text { Background }\end{array}$ & $\begin{array}{c}\text { Highest } \\
\text { Detection }\end{array}$ \\
\hline \multicolumn{5}{|l|}{ RADIOISOTOPES (pCi/g) } \\
\hline Bismuth-214 & 0.00786 & $* 0.91$ & $1 / 3$ & 0.92 \\
\hline Potassium-40 & 0.0771 & $* 1.59$ & $1 / 3$ & 17.31 \\
\hline Gross Alpha & - & $\times 20$ & $1 / 21$ & 24.02 \\
\hline Non-Volatile Beta & $\cdots$ & $\times 50$ & $4 / 28$ & 253.75 \\
\hline \multicolumn{5}{|l|}{ VOCs $\mu \mathrm{g} / \mathrm{kg}$} \\
\hline Acetone & 780 & 19.34 & $4 / 25$ & 449.00 \\
\hline Carbon disulfide & 780 & 0.47 & $6 / 25$ & 7.60 \\
\hline Chioromethane & 85 & 10.78 & $2 / 25$ & 28.80 \\
\hline Methyl ethyl ketone & 4700 & 49.38 & $19 / 20$ & 600.10 \\
\hline Methyl isobutyl ketone & 390 & 8.72 & $7 / 20$ & 19.50 \\
\hline \multicolumn{5}{|l|}{ SVOCs $\mu \mathrm{g} / \mathrm{kg}$} \\
\hline Bis(2-ethylhexyl) phthalate & 46 & 370 & $6 / 19$ & \\
\hline \multicolumn{5}{|l|}{ PESTICIDES \& PCBS $\mu \mathrm{g} / \mathrm{kg}$} \\
\hline \multicolumn{5}{|c|}{ No values observed above the $2 X$ average background values } \\
\hline & & & $\because$ & \\
\hline DIOXINS, FURANS $1 \mathrm{~g} / \mathrm{kg}$ & & & + & \\
\hline \multicolumn{3}{|c|}{ No values observed above the $2 \mathrm{X}$ average background values } & $\cdot$ & \\
\hline \multicolumn{4}{|c|}{ TOTAL PETROLEUM HYDROCARBONS mg/kg } & \\
\hline \multicolumn{5}{|l|}{ Not Analyzed } \\
\hline \multicolumn{5}{|l|}{ METALS mg/kg } \\
\hline Aluminum & 23000 & 6124 & $9 / 20$ & 16100.00 \\
\hline Arsenic & 0.37 & 1.11 & $8 / 20$ & 3.00 \\
\hline Barium & 550 & 18.72 & $2 / 20$ & 32.32 \\
\hline Cadmium & 3.9 & 0.56 & $1 / 25$ & 0.73 \\
\hline Calcium & 820 & 749.2 & $1 / 20$ & 994.91 \\
\hline Chromium & 39 & 10.72 & $11 / 20$ & 62.60 \\
\hline Copper & 290 & 3 & $4 / 20$ & 4.50 \\
\hline Iron & 24000 & 16923.8 & $9 / 20$ & 35800.00 \\
\hline Lead & 5 & 5.65 & $3 / 25$ & 13.50 \\
\hline Magnesium & 520 & 748.8 & $1 / 20$ & 637.97 \\
\hline Manganese & 39 & 22.2 & $5 / 25$ & 115.00 \\
\hline Nickel & 160 & 3.06 & $3 / 25$ & 3.90 \\
\hline Potassium & 940 & 748.8 & $3 / 20$ & 1062.31 \\
\hline Selenium & 39 & 0.34 & $3 / 25$ & 0.47 \\
\hline Silver & 39 & 0.79 & $1 / 25$ & 0.88 \\
\hline Thallium & & 0.78 & $1 / 20$ & 63.75 \\
\hline Vanadium & 55 & 38.08 & $8 / 20$ & 82.90 \\
\hline Zinc & 2300 & 4.4 & $5 / 20$ & 78.42 \\
\hline
\end{tabular}

Legend:

Frequency: $5 / 12=$ Detects above $2 \mathrm{X}$ average background values/total number of samples analyzed.

PRGs - Risk-based (1E-06) Preliminary Remedial Goals

2 The screening levels of 20 and $50 \mathrm{pCi} / \mathrm{g}$ for gross alpha and non-volatile beta, respectively, are based on site-specific background samples and the presence of naturally occurring radionuclides (see Section 2.6.3). Chemical Class headings labeled as "Not Analyzed" means that none of the constituents in the chemical class were analyzed. 
TABLE 1-5

LAOCB PIPELINE

SUMMARY OF DETECTED CONSTITUENTS IN SOIL

\begin{tabular}{|c|c|c|c|c|}
\hline Analyte & $\begin{array}{c}\text { USEPA } \\
\text { Region } 3 \\
\text { PRGs } \\
\end{array}$ & $\begin{array}{c}2 \text { X Average } \\
\text { Background } \\
\text { Value } \\
\end{array}$ & $\begin{array}{c}\text { Frequency of Detects } \\
\text { Above } 2 \text { X Average } \\
\text { Background }\end{array}$ & $\begin{array}{c}\text { Highest } \\
\text { Detection }\end{array}$ \\
\hline \multicolumn{5}{|c|}{ RADIOISOTOPES (pCi/g) } \\
\hline Potassium-40 & 0.0771 & $* 1.59$ & $1 / 2$ & 2.55 \\
\hline Non-Volatile Beta & $\ldots$ & 250 & $3 / 17$ & 289.78 \\
\hline \multicolumn{5}{|l|}{ VOCs $\mu \mathrm{g} / \mathrm{kg}$} \\
\hline Acetone & 780 & 19.34 & $1 / 41$ & 133.80 \\
\hline Carbon disulfide & 780 & 0.47 & $4 / 41$ & 7.00 \\
\hline Methyl ethyl ketone & 4700 & 49.38 & $13 / 41$ & 645.50 \\
\hline Methyl isobutyl ketone. & 390 & 8.72 & $5 / 41$ & 33.20 \\
\hline Toluene & 1600 & 3.24 & $4 / 41$ & 24.10 \\
\hline \multicolumn{5}{|l|}{ SVOCs $\mu \mathrm{g} / \mathrm{kg}$} \\
\hline \multicolumn{5}{|c|}{ No values observed above the $2 X$ average background values } \\
\hline \multirow{2}{*}{\multicolumn{5}{|c|}{ PESTICIDES \& PCBs $\mu \mathrm{g} / \mathrm{kg}$}} \\
\hline & & & & \\
\hline \multicolumn{5}{|c|}{ No values observed above the $2 \mathrm{X}$ average background values } \\
\hline \multicolumn{5}{|c|}{ DIOXINS, FURANS $\mu \mathrm{g} / \mathrm{kg}$} \\
\hline \multicolumn{5}{|l|}{ Not Analyzed } \\
\hline \multicolumn{5}{|c|}{ TOTAL PETROLEUM HYDROCARBONS mg/kg } \\
\hline \multicolumn{5}{|l|}{ Not Analyzed } \\
\hline & & & & \\
\hline Aluminum & \multicolumn{4}{|c|}{ METALS mg/kg } \\
\hline Arsenic & 0.37 & 1.11 & $8 / 41$ & 2.50 \\
\hline Barium & 550 & 18.72 & $11 / 41$ & 44.12 \\
\hline Beryllium & 0.15 & 0.34 & $-1 / 41$ & 11.50 \\
\hline Cadmium & 3.9 & 0.56 & $1 / 41$ & 8.79 \\
\hline Calcium & 820 & 749.20 & $4 / 41$ & 2714.05 \\
\hline Chromium & 39 & 10.72 & $21 / 41$ & 24.01 \\
\hline Cobalt & 4700 & 3.68 & $1 / 41$ & 13.15 \\
\hline Copper & 290 & 3.00 & $6 / 41$ & 62.46 \\
\hline Iron & 24000 & 16923.80 & $12 / 41$ & 25400.00 \\
\hline Lead & 5 & 5.65 & $7 / 41$ & 60.01 \\
\hline Magnesium & 520 & 748.80 & $2 / 41$ & 650.09 \\
\hline Manganese & 39 & 22.20 & $17 / 41$ & 220.07 \\
\hline Potassium & 940 & 748.80 & $7 / 41$ & 1085.87 \\
\hline Silver & 39 & 0.79 & $1 / 41$ & 0.80 \\
\hline Thallium & & 0.78 & $2 / 41$ & 52.57 \\
\hline Vanadium & 55 & 38.08 & $12 / 41$ & 62.60 \\
\hline Zinc & 2300 & 4.40 & $16 / 41$ & 298.53 \\
\hline
\end{tabular}

Legend:

Frequency: $5 / 12$ = Detects above $2 X$ average background values/total number of samples analyzed.

* Background value is established from an altemate background soil boring (LAOCB-20). Samples collected from the designated background boring were not speciated beyond Gross Alpha and Non-volatile Beta. PRGs - Risk-based (IE-06) Preliminary Remedial Goals

2 The screening levels of 20 and $50 \mathrm{pCi} / \mathrm{g}$ for gross alpha and non-volatile beta, respectively, are based on site-specific background samples and the presence of naturally occurring radionuclides (see Section 2.6.3).

Chemical Class headings labeled as "Not Analyzed" means that none of the constituents in the chemical class were analyzed. 
TABLE 1-6

LAOCB STANDING WATER

SUMMARY OF DETECTED CONSTITUENTS

\begin{tabular}{|c|c|c|c|}
\hline Analyte & $\begin{array}{c}\text { EPA } \\
\text { MCLs }\end{array}$ & $\begin{array}{l}\text { USEPA } \\
\text { Region } 3 \\
\text { PRGs }\end{array}$ & $\begin{array}{c}\text { Maximum Detection } \\
\text { of Samples } \\
\text { Analyzed }\end{array}$ \\
\hline \multicolumn{4}{|l|}{ RADIOISOTOPES (pCi/mL) } \\
\hline Bismuth-214 & & $5.90 \mathrm{E}-05$ & 0.3 \\
\hline Cesium-137 & & $4.80 \mathrm{E}-04$ & 0.25 \\
\hline Cobalt -60 & & $8.20 \mathrm{E}-05$ & 0.5 \\
\hline Potassium-40 & & $1.20 \mathrm{E}-03$ & 1.2 \\
\hline Strontium-90 & & $1.80 \mathrm{E}-04$ & 170 \\
\hline Thallium-208 & & 1.29 & 0.5 \\
\hline Tritium & 20.0 & & 8.6 \\
\hline Gross Alpha & 15.0 & & 2.3 \\
\hline Non-Volatile Beta & & & 86.25 \\
\hline \multicolumn{4}{|l|}{ VOCs } \\
\hline 1,2-Dichloropropane & 5.0 & 0.16 & 0.2 \\
\hline Benzene & 5.0 & 0.087 & 0.4 \\
\hline Bromomethane (Methyl bromide) & & 0.87 & 1.3 \\
\hline Carbon disulfide & & 2.1 & 0.4 \\
\hline Dichloromethane (Methylene chloride) & 5.0 & 4.1 & 0.4 \\
\hline Styrene & 100 & 100 & 0.2 \\
\hline \multicolumn{4}{|l|}{ SVOCs } \\
\hline \multicolumn{4}{|l|}{ Not Analyzed } \\
\hline \multicolumn{4}{|l|}{ PESTICIDES \& PCBS } \\
\hline \multicolumn{4}{|l|}{ Not Analyzed } \\
\hline \multicolumn{4}{|l|}{ DIOXINS \& FURANS } \\
\hline \multicolumn{4}{|l|}{ Not Analyzed } \\
\hline & & & \\
\hline \multicolumn{4}{|l|}{ TPH } \\
\hline \multicolumn{4}{|l|}{ Not Analyzed } \\
\hline \multicolumn{4}{|l|}{ METALS mg/kg } \\
\hline Aluminum & & 11000 & 238 \\
\hline Barium & 2000 & 260 & 11.8 \\
\hline Cadmium & 0.005 & 1.8 & 0.5 \\
\hline Calcium & - & $\cdots$ & 2740 \\
\hline Iron & 300 & & 1440 \\
\hline Manganese & 50 & 18 & 1900 \\
\hline Nickel & 100 & 73 & 32.4 \\
\hline Potassium & -- & & 8170 \\
\hline Zinc & 1100 & 1100 & 186 \\
\hline
\end{tabular}

Legend:

MCLs - Maximum Contaminant Levels

PRGs - Preliminary Remediation Goals

Chemical class headings labeled as "Not Analyzed" mean that none of the constituents in the chemical class were analyzed. 
TABLE 1-7

RADIOACTIVE ISOTOPE HALF-LIVES

L-AREA OIL AND CHEMICAL BASIN

SAVANNAH RIVER SITE

\begin{tabular}{|c|c|}
\hline Isotope & Half-Life \\
\hline Americium-241 & 432 years \\
\hline Antimony-125 & 2.77 years \\
\hline Cerium-144 & 284 days \\
\hline Cesium-137 & 30.0 years \\
\hline Cobalt-57 & 270.9 days \\
\hline Cobalt -60 & 5.27 years \\
\hline Curium-244 & 18.1 years \\
\hline Europium-152 & 13.3 years \\
\hline Europium-154 & 8.8 years \\
\hline Lead-212 & 10.64 hours \\
\hline Plutonium-238 & 87.74 years \\
\hline Plutonium-239 & 24,065 years \\
\hline Potassium-40 & $1.3 \times 10^{9}$ years \\
\hline Ruthenium-106 & 368.2 days \\
\hline Strontium-90 & 29.1 years \\
\hline Thorium-234 & 24.1 days \\
\hline Tritium & 12.4 years \\
\hline Uranium-234 & $2.45 \times 10^{5}$ years \\
\hline Uranium-235 & $7.0 \times 10^{8}$ years \\
\hline Uranium-238 & $4.5 \times 10^{9}$ years \\
\hline
\end{tabular}

Source: Browne and Firestone, 1986 
TABLE 1-8

SUMMARY OF HUMAN HEALTH CHEMICALS OF CONCERN

L-AREA OIL AND CHEMICAL BASIN

SAVANNAH RIVER SITE

\begin{tabular}{|c|c|c|}
\hline Chemical Type & Chemicals of Conce & \\
\hline \multirow[t]{8}{*}{ Nonradionuclides } & \multicolumn{2}{|l|}{ Aluminum } \\
\hline & \multicolumn{2}{|l|}{ Chromium } \\
\hline & \multicolumn{2}{|l|}{ Lead } \\
\hline & \multicolumn{2}{|l|}{ Cadmium } \\
\hline & \multicolumn{2}{|l|}{ Nickel } \\
\hline & Thallium & - \\
\hline & \multicolumn{2}{|l|}{ Arsenic } \\
\hline & \multicolumn{2}{|l|}{ Vanadium } \\
\hline \multirow[t]{14}{*}{ Radionuclides } & \multicolumn{2}{|l|}{ Americium-241 } \\
\hline & \multicolumn{2}{|l|}{ Antimony-125 } \\
\hline & \multicolumn{2}{|l|}{ Cesium-137 } \\
\hline & \multicolumn{2}{|l|}{ Cobalt -60} \\
\hline & \multicolumn{2}{|l|}{ Curium-244 } \\
\hline & \multicolumn{2}{|l|}{ Europium-152 } \\
\hline & \multicolumn{2}{|l|}{ Europium-154 } \\
\hline & \multicolumn{2}{|l|}{ Plutonium-238 } \\
\hline & \multicolumn{2}{|l|}{ Plutonium-239 } \\
\hline & \multicolumn{2}{|l|}{ Potassium -40} \\
\hline & \multicolumn{2}{|l|}{ Strontium-90 } \\
\hline & \multicolumn{2}{|l|}{ Uranium-234 } \\
\hline & \multicolumn{2}{|l|}{ Uranium-235 } \\
\hline & \multicolumn{2}{|l|}{ Uranium-238 } \\
\hline
\end{tabular}




\subsection{TECHNOLOGY IDENTIFICATION AND SCREENING}

This section presents the unit-specific remedial action objectives for media of concern at the LAOCB. As discussed in Section 1.0, remediation of groundwater at the LAOCB will be addressed as appropriate in a separate document.

Unit-specific remedial action objectives are based on the results of the BRA and on the evaluation of applicable or relevant and appropriate requirements (ARARs) and "to-beconsidered" (TBC) information. ARARs and TBC information are defined and discussed further in Sections 2.3.1.1 and 2.3.1.2, respectively.

\section{$2.1 \quad$ Introduction}

The purpose of this identification and screening process is to identify a range of suitable remedial action technologies and remedial options that can be assembled into corrective measures/remedial alternatives capable of mitigating contamination at the LAOCB. EPA has established a structured process for identifying and screening relevant technologies for purposes of remediation. The goal of the remedy selection process established by EPA is to select corrective measures/remedial actions that protect human health and the environment, that maintain protection over time, and that minimize contaminant (or waste) mobility, toxicity, or volume through treatment, when possible [CERCLA 300.430 (a)(1)(I)]. The CMS/FS process ensures that appropriate corrective measures/remedial options are developed and evaluated, and that pertinent information required to select a recommended remediation approach is presented.

The NCP specifies six expectations for developing remedial alternatives [CERCLA 300.430 (a)(1)(iii)]. These include:

- using treatment, whenever practicable, to address principal threats posed by the unit 
- using engineering controls for waste that poses a relatively low long-term risk or when treatment is impractical

- combining methods, such as treatment, with engineering controls to protect human health and the environment

- $\quad$ supplementing engineering controls with institutional controls, as appropriate, for shortand long-term management to prevent or limit exposure

- using innovative technology when practical

Selecting a response action proceeds in a series of steps designed to reduce the universe of potential technologies from which site-wide alternatives may be developed. The response action selection process as defined in the NCP is outlined in Figure 2-1.

\subsection{Remedial Action Objectives}

Remedial action objectives address unit-specific contaminants, media of concern, potential exposure pathways, and remediation goals. The remedial action objectives are based on the nature and extent of contamination, threatened resources, and the potential for human and environmental exposure. Initially, preliminary remediation goals are developed based upon ARARs, or other information from the RFI/RI and BRA. These goals may be refined, as necessary, as more information concerning the unit and potential remedial technologies becomes available. Final remediation goals will be determined when the remedy is selected and they shall establish acceptable exposure levels that are protective of human health and the environment [CERCLA 300.430 (e)(2)(I)]. For example, a remedial action objective of remedial action involving off-unit disposal would include the reduction of contaminant concentrations in the impacted media to levels that are protective of human health and the environment, whereas, a remedial action objective involving on-unit containment would include the elimination of direct exposure pathways to potential receptors. 


\subsubsection{Applicable or Relevant and Appropriate Requirements (ARARs)}

Section 121(d) of the CERCLA of 1980, as amended by the SARA of 1986, requires that remedial actions comply with requirements or standards set forth under Federal and State environmental laws. ARARs are typically evaluated under the categories of action-specific, location-specific, and chemical-specific ARARs.

As mandated by [CERCLA 121(d)(2)(A)], remedies must consider "any promulgated standard, requirements, criteria, or limitation under a State environmental or facility siting law that is more stringent than any Federal standard, requirement, criteria, or limitation" if the former is applicable or relevant and appropriate to the site and associated remedial activities. SARA requires that the remedial action for a waste unit meet all ARARs unless a waiver is invoked. Five circumstances under which a waiver may be invoked are:

- the remedial action is an interim measure where the final remedy will attain the ARAR upon completion

- compliance will result in greater risk to human health and the environment than other options

- $\quad$ compliance is technically impracticable

- an alternative remedial action will attain the equivalent of the ARAR

- for State requirements, the State has not consistently applied the requirement in similar circumstances

In addition to ARARs, many Federal and State environmental and public health programs also develop TBC criteria, guidance, and proposed standards that are not legally binding, but that may provide useful information or recommended procedures (EPA, 1988c). These TBCs are not potential ARARs but are reviewed along with ARARs and considered when setting remediation objectives (e.g., cleanup goals). To-be-considered information is further defined in Section. 2.2.1.2. 


\subsubsection{Definition of ARARs}

Potential ARARs may be classified as either applicable or relevant and appropriate. Applicable requirements are those cleanup standards, standards of control, and other substantive environmental protection requirements, criteria, or limitations promulgated under Federal or State law that specifically address a hazardous substance, pollutant, contaminant, remedial action, location, or other circumstance at a CERCLA site (EPA, 1988c).

Relevant and appropriate requirements are those cleanup standards, standards of control, and other substantive environmental protection requirements, criteria, or limitations promulgated under Federal or State law that, while not applicable to a hazardous substance, pollutant, contaminant, remedial action, location, or other circumstance at a CERCLA site, address problems or situations sufficiently similar to those encountered at the CERCLA site so that their use is well suited to the particular site (EPA, 1988c).

\subsubsection{To-Be-Considered Information}

To-be-considered requirements (TBCs) are non-promulgated advisories or guidance issued by Federal or State government that are not legally binding and do not have the status of potential ARARs. However, TBCs may be considered along with ARARs in determining the necessary level of cleanup for protection of health or the environment. For example, DOE Orders are not ARARs since they have not been formally promulgated under Federal or State law; however, these Orders should be considered along with ARARs in determining remedial action objectives. DOE Orders are legally binding for DOE and all of its contractors through the Price Anderson Amendments Act of 1988, which is the amendment to the Atomic Energy Act. 


\subsubsection{Types of ARARs}

ARARs are conveniently classified as being either action-specific, chemical-specific, or locationspecific to further clarify how to identify and comply with environmental requirements. These types of ARARs are described in the following subsections.

\section{Action-Specific ARARs}

Action-specific requirements set controls or restrictions on the design, performance, and other aspects of implementation of specific remedial activities. Examples include RCRA regulations for off-site disposal of hazardous residuals and the Clean Water Act standards for discharge of treated groundwater. Because action-specific ARARs apply to discrete remedial activities, their evaluation is deferred until Section 4.0 when detailed analysis is performed on each retained alternative.

SARA Section 121(e) exempts any onsite CERCLA response action from having to obtain a Federal, State and/or local permit. CERCLA on-site response actions must, however, comply with the substantive aspects of these requirements (EPA, 1988c).

\section{Location-Specific ARARs}

Location-specific ARARs must consider Federal, State, and local requirements that reflect the physiographical and environmental characteristics of the unit or the immediate area. Remedial actions may be restricted or precluded based on the location or characteristics of the unit and the resulting requirements. Potential location-specific ARARs are evaluated for the LAOCB in Table 2-1. 


\section{Chemical-Specific ARARs}

Chemical-specific ARARs are media-specific concentration limits promulgated under Federal or State law. The NCP requires, where possible, the development of health-based site-specific levels for chemicals or media where such limits do not exist and there is a concern with their potential health or environmental impacts. Potential chemical-specific ARARs for sludge and subsoil are evaluated as part of the development of remedial action objectives in Section 2.2.2.3. Potential chemical-specific ARARs for vegetation are evaluated in Section 2.3.4.

\subsubsection{Remedial Action Objectives for $L A O C B$ and $L A O C B$ Pipeline Soil}

Sludge/soil at the LAOCB are contaminated with radionuclides and metals from the processes formerly conducted at the facility. The BRA indicated that LAOCB sludge/soil [0-0.6 m (0-2 $\mathrm{ft})]$ pose a potential threat to human health through exposure to the following primary and secondary COCs:

\section{Primary COCs (LAOCB Sludge/Soil)}

- Americium-241

- Cesium-137

- Curium-244

- Plutonium-238/239

- Strontium-90

- Aluminum

- Chromium

- Nickel
- Antimony-125

- Cobalt-60

- Europium-152/154

- Potassium-40

- Uranium-234/235/238

- Cadmium

- Lead

- Vanadium

\section{$\underline{\text { Secondary COCs (LAOCB Sludge/Soil) }}$}

- Actinium-228

- Bismuth-214

- Beryllium
- Thallium-208

- Potassium-40 
The BRA determined that LAOCB pipeline soil pose a potential threat to human health through exposure to the following primary and secondary COCs:

\section{Primary COCs (Pipeline Soils)}

- Aluminum

- Lead
- Arsenic

- Thallium

\section{Secondary COCs (Pipeline Soils)}

- Beryllium

- Thallium-208

Radionuclides are unique contaminants with a very limited selection of remedial responses/technologies that include:

- $\quad$ No Action (not appropriate)

- Containment (capping)

- Treatment/Stabilization-Solidification

- Off-Unit Disposal

Since all of the risk in the LAOCB is posed by radionuclides and metals with very similar physical and chemical properties, these technologies, or any combination of them, are applicable to the treatment of all COCs (primary and secondary).

\subsubsection{Identification of Chemical-Specific ARARs}

The following subsections discuss potential chemical-specific ARARs for the LAOCB.

\section{Toxic Substances Control Act (TSCA)}

The only nonradioactive chemical-specific ARARs for soil under Federal and South Carolina regulations are for PCBs. ARARs for PCBs are governed by the Toxic Substances Control Act 
(TSCA; 40 CFR Part 761). For non-restricted access areas (e.g., residential), the PCB cleanup standard is $10 \mathrm{mg} / \mathrm{kg}$ by weight, provided that the soil is excavated to a minimum depth of $25 \mathrm{~cm}$ (10 in.) and that the excavated soil is replaced with clean soil (i.e., soil containing less than 1 $\mathrm{mg} / \mathrm{kg}$ PCBs). Based on the disposal history of the LAOCB and the fact that there were no significant PCB concentrations detected in LAOCB sludge and subsoil samples, TSCA is not an ARAR.

Uranium Mill Tailings Radiation Control Act (UMTRCA) and DOE Order 5400.5

Of the radioactive constituents detected in soil, ${ }^{226} \mathrm{Ra},{ }^{228} \mathrm{Ra},{ }^{230} \mathrm{Th}$, and ${ }^{232} \mathrm{Th}$ are regulated through an ARAR and a TBC. These are:

- 40 CFR 192 Subparts A through E - Health and Environmental Protection Standards for Uranium and Thorium Mill Tailings

- DOE Order 5400.5 - Radiation Protection of the Public and the Environment

Both 40 CFR 192 and DOE Order 5400.5 specify that the concentration of ${ }^{226} \mathrm{Ra},{ }^{228} \mathrm{Ra},{ }^{230} \mathrm{Th}$, and ${ }^{232} \mathrm{Th}$ in soil averaged over an area of $100 \mathrm{~m}^{2}\left(1,076 \mathrm{ft}^{2}\right)$ shall not exceed the background level by more than:

- $\quad 5 \mathrm{pCi} / \mathrm{g}$ over the first $15 \mathrm{~cm}$ (5.9 in.) of soil below the surface

- $\quad 15 \mathrm{pCi} / \mathrm{g}$ averaged over $14.9 \mathrm{~cm}(5.9 \mathrm{in}$.)-thick layers of soil more than $14.9 \mathrm{~cm}(5.9 \mathrm{in})$. below the surface

The objective of remedial actions (if warranted) based on regulated levels of radium and thorium in soil would be to reduce radon and decay product concentrations, including background, so as not to exceed an average annual release rate of $20 \mathrm{pCi} / \mathrm{m}^{2} / \mathrm{s}$ or increase the annual concentration of radon- $222\left({ }^{222} \mathrm{Rn}\right)$ in air at any location outside the contaminated area by more than $0.5 \mathrm{pCi} / 1$. 
Sediment samples collected from the basin were not analyzed for alpha emitting $\mathrm{Ra}$ isotopes. Therefore, it is unknown whether the level of $\mathrm{Ra}$ in sediment at the source area melts the specified ARAR and TBC Ra concentrations.

No detectable activities of ${ }^{230} \mathrm{Th}$ or ${ }^{232} \mathrm{Th}$ were present in the basin sediment. Soil in these areas meet the ARAR and specified levels in DOE Order 5400.5.

\subsubsection{To-Be-Considered Information}

\section{$\underline{\text { RCRA Subpart S Proposed Media Protection Standards }}$}

Action levels and media protection standards have been proposed by EPA as part of the RCRA Corrective Action Program (55 FR 30798) but have not yet been promulgated under Federal or State law. Action levels are guidelines that are risk-based limits to be used in determining the need for corrective measures at RCRA SWMUs. Media cleanup standards represent constituent concentrations in soil, groundwater, surface water, and air that remedies must achieve to comply with standards for remedies under 40 CFR $264.524(\mathrm{a})(2)$. When formally promulgated, these media cleanup standards may become ARARs for CERCLA remedial actions at SRS. Currently, however, the media cleanup standards are TBC information in terms of ARARs. Additionally; RCRA Subpart $\mathrm{S}$ does not provide soil media cleanup standards for radionuclides or non-radioactive metals.

\section{$\underline{\text { Remedial Goal Options }}$}

Human health risk-based Remedial Goal Options (RGOs) are calculated for COCs which are primary contributors of potential risk and/or adverse effects for the current and future use scenarios using standard EPA default equations. Risk-based RGOs are estimated only for pathways exceeding a potential carcinogenic risk of $1 \mathrm{E}-04$ and HIs exceeding unity. RGOs are 
not required remediation goals or standards but are developed as part of the BRA to provide a range of risk-based cleanup goals for consideration in the $\mathrm{CMS} / \mathrm{FS}$ process.

During the development of the BRA, it was determined that radionuclides and metals represent 100 percent of the risk above $1 \mathrm{E}-06$ for all applicable receptors. Specifically, radionuclides represent greater than 99 percent of the total unit risk. The predominant risk drivers for current and hypothetical receptors are direct radiation from cobalt- 60 and cesium-137 and ingestion of soils from chromium and lead. Both the future on-unit resident and the future on-unit worker has significant risk from exposure to these radionuclides and nonradionuclides (metals). Table 2-2 provides risk-based RGOs for each primary COC (>1E-04) in soil of the LAOCB and pipeline area. RGOs were not developed for secondary COCs (1E-04 to $1 \mathrm{E}-06)$; however, the remedial alternatives are applicable to both the primary and secondary COCs. Discussed below are the RGOs for the COCs which are considered the primary contributors to potential risk within an exposure pathway posing potential risk.

Basin Soil. Direct radiation from radioactive constituents within the seepage basin soil also pose a carcinogenic risk of greater than 1E-04 to the hypothetical future industrial worker and the hypothetical future resident. The primary contributors to the risk are ${ }^{60} \mathrm{Co}$ and ${ }^{137} \mathrm{Cs}$. The estimated RGOs for ${ }^{60} \mathrm{Co}$ to achieve the $1 \mathrm{E}-06$ risk level are:

- $\quad 7.5 \mathrm{E}-02 \mathrm{pCi} / \mathrm{g}$ for the hypothetical future worker

- $\quad 8.8 \mathrm{E}-03 \mathrm{pCi} / \mathrm{g}$ for the hypothetical future resident

The estimated RGOs for ${ }^{137} \mathrm{Cs}$ to achieve the $1 \mathrm{E}-06$ risk level are:

- $\quad 0.32 \mathrm{pCi} / \mathrm{g}$ for the future worker

- $\quad 3.7 \mathrm{E}-02 \mathrm{pCi} / \mathrm{g}$ for the future resident

The estimated RGOs for ${ }^{60} \mathrm{Co}$ to achieve the $1 \mathrm{E}-04$ risk level are : 
- $\quad 7.5 \mathrm{pCi} / \mathrm{g}$ for the hypothetical future worker

- $\quad 0.88 \mathrm{pCi} / \mathrm{g}$ for the hypothetical future resident

The estimated $\mathrm{RGOs}$ for ${ }^{137} \mathrm{Cs}$ to achieve the $1 \mathrm{E}-04$ risk level are :

- $\quad 32 \mathrm{pCi} / \mathrm{g}$ for the hypothetical future worker

- $\quad 3.7 \mathrm{pCi} / \mathrm{g}$ for the hypothetical future resident

Background concentrations of ${ }^{60} \mathrm{Co}$ at the unit are below detectable levels. Therefore, the concentration-based remediation goals for ${ }^{60} \mathrm{Co}$ would be to achieve the concentration at which the $1 \mathrm{E}-04$ to $1 \mathrm{E}-06$ risk levels are estimated for the future land use scenarios (i.e.e., $0.88 \mathrm{pCi} / \mathrm{g}$ to $8.8 \mathrm{E}-03 \mathrm{pCi} / \mathrm{g}$ for residential use and 7.5 to $7.5 \mathrm{E}-02$ for industrial use). The maximum detected concentration of ${ }^{60} \mathrm{Co}$ in the basin soil is $5,242 \mathrm{pCi} / \mathrm{g}$.

Background concentrations of ${ }^{137} \mathrm{Cs}$ detected at the boundary of SRS range from $0.58 \mathrm{pCi} / \mathrm{g}$ to $0.81 \mathrm{pCi} / \mathrm{g}$ (Looney, et al., 1990). The RGOs for ${ }^{137} \mathrm{Cs}$ at the $1 \mathrm{E}-06$ and $1 \mathrm{E}-05$ risk level under either the future worker or the future resident scenario are less than background levels. Therefore, the proposed concentration-based remediation level for ${ }^{137} \mathrm{Cs}$ in LAOCB soil to achieve future unrestricted land use at the unit is the identified background concentrations. Alternative concentration-based remediation goals for ${ }^{137} \mathrm{Cs}$ in soil would be to achieve the concentration at which 1E-04 risk levels are estimated for the future land use scenarios (i.e., 3.7 $\mathrm{pCi} / \mathrm{g}$ for residential use and $32 \mathrm{pCi} / \mathrm{g}$ for industrial use). The maximum detected concentrations of ${ }^{137} \mathrm{Cs}$ in the basin soil is $1,154 \mathrm{pCi} / \mathrm{g}$.

Ingestion of soil from the $\mathrm{LAOCB}$ has a radiological risk of $2 \mathrm{E}-04$ due principally to: ${ }^{241} \mathrm{Am}$, ${ }^{90} \mathrm{Sr}$, and ${ }^{239} \mathrm{Pu}$. Estimated radiological risk levels for ingestion of fruit produce (2E-03), leafy produce $(9 \mathrm{E}-04)$, and tuberous produce $(3 \mathrm{E}-03)$ were due to ${ }^{90} \mathrm{Sr}$ and ${ }^{137} \mathrm{Cs}$. The estimated RGOs to achieve the $1 \mathrm{E}-06$ risk level for, ${ }^{241} \mathrm{Am},{ }^{137} \mathrm{Cs},{ }^{239} \mathrm{Pu}$, and ${ }^{90} \mathrm{Sr}$ are $12 \mathrm{pCi} / \mathrm{g}, 0.32 \mathrm{pCi} / \mathrm{g}, 14$ 
$\mathrm{pCi} / \mathrm{g}$, and $90 \mathrm{pCi} / \mathrm{g}$, respectively, for the future worker scenario. The estimated $\mathrm{RGOs}$ to achieve the $1 \mathrm{E}-06$ level for ${ }^{241} \mathrm{Am},{ }^{137} \mathrm{Cs},{ }^{239} \mathrm{Pu}$, and ${ }^{90} \mathrm{Sr}$ are $2.6 \mathrm{pCi} / \mathrm{g}, 3.7 \mathrm{E}-02 \mathrm{pCi} / \mathrm{g}, 3.4 \mathrm{pCi} / \mathrm{g}$, and $0.39 \mathrm{pCi} / \mathrm{g}$, respectively, for the future resident scenario. The maximum concentrations of ${ }^{241} \mathrm{Am},{ }^{137} \mathrm{Cs},{ }^{239} \mathrm{Pu}$, and ${ }^{90} \mathrm{Sr}$ are $805 \mathrm{pCi} / \mathrm{g}, 1,154 \mathrm{pCi} / \mathrm{g}, 811 \mathrm{pCi} / \mathrm{g}$, and $2,707 \mathrm{pCi} / \mathrm{g}$, respectively (Table 2-2).

The HIs for ingestion of LAOCB soil are greater than one for the resident, adult and child, exposure $(\mathrm{HI}=5)$ and also the resident, child only, exposure $(\mathrm{HI}=20) . \mathrm{Cr}$ and $\mathrm{Pb}$ are primarily responsible for these hazards. Dermal exposures to soil at the LAOCB have an adult and child $\mathrm{HI}$ of 1 and a child only HI of 2. Cr is responsible for the hazard. For ingestion of homegrown produce from the LAOCB, HIs are greater than or equal to one in the following scenarios: leafy produce ingestion (adult and child $\mathrm{HI}=1$ ), leafy produce ingestion (child only $\mathrm{HI}=2$ ), and tuberous produce ingestion (child only $\mathrm{HI}=1$ ). $\mathrm{Cr}, \mathrm{Cd}$, and $\mathrm{Pb}$ are primarily responsible for these hazards. The estimated RGOs for $\mathrm{Cr}, \mathrm{Cd}$, and $\mathrm{Pb}$ to achieve an HQ of unity are $340 \mathrm{mg} / \mathrm{kg}, 12$ $\mathrm{mg} / \mathrm{kg}$, and $28 \mathrm{mg} / \mathrm{kg}$, respectively. The maximum concentrations of $\mathrm{Cr}, \mathrm{Cd}$, and $\mathrm{Pb}$ in the basin soil are $5,250 \mathrm{mg} / \mathrm{kg}, 12.3 \mathrm{mg} / \mathrm{kg}$, and $243 \mathrm{mg} / \mathrm{kg}$, respectively. Other COCs which contributed to the potential hazards, although to a lesser extent, include $\mathrm{Al}, \mathrm{Ni}$, and $\mathrm{V}$ (Table 2-2).

Soil Adjacent to the Basin. Carcinogenic risks for future exposure scenarios to soil outside the basin area were within the EPA acceptable risk range. Hazard estimates for soil adjacent to the LAOCB had no HIs greater than or equal to one. Therefore, no COCs are identified for soil adjacent to the unit.

LAOCB Pipeline Soil. The radiological risk from exposure to soil $0-3.7 \mathrm{~m}(0-12 \mathrm{ft})$ at the LAOCB pipelines is less than 1E-06 for all future land use pathways quantified with the exception of external exposure to gamma radiation, which is within the EPA risk range of 1E-04 to $1 \mathrm{E}-06$. The external exposure risk from pipeline soils is attributed to ${ }^{208} \mathrm{Tl} .{ }^{208} \mathrm{Tl}$ has been 
shown to be a naturally occurring isotope in the Thorium decay series that is prevalent in SRS soils.

Ingestion of LAOCB pipelines soil from 0-1.2 $\mathrm{m}(0-4 \mathrm{ft})$ depth has an $\mathrm{HI}$ of 3 for adult and child residents and an $\mathrm{HI}$ of 9 for child only exposures. Thallium is the sole contributor to this hazard. LAOCB pipelines soil from a depth of $0-3.7 \mathrm{~m}(0-12 \mathrm{ft})$ has an $\mathrm{HI}$ greater than or equal to one for adult and child exposure through ingestion $(\mathrm{HI}=1)$ and child only exposure through ingestion $(\mathrm{HI}=4)$. $\mathrm{Tl}$ and $\mathrm{Pb}$ are responsible for the majority of this hazard. The estimated RGOs for $\mathrm{Tl}$ and $\mathrm{Pb}$ to achieve $\mathrm{HQs}$ of one are $6.1 \mathrm{mg} / \mathrm{kg}$ and $28 \mathrm{mg} / \mathrm{kg}$, respectively. The maximum concentrations of thallium and lead in the LAOCB pipelines soil are $53 \mathrm{mg} / \mathrm{kg}$ and $60 \mathrm{mg} / \mathrm{kg}$, respectively. Al and As were also identified as COCs which contributed to the potential hazard, however, to a lesser extent (see Table 2-2).

LAOCB Pipelines. The LAOCB pipelines have not been sampled for radionuclide concentrations. Therefore, a BRA was not conducted for the pipelines and RGOs could not be developed.

\subsubsection{Summary of Remedial Action Objectives for Soil}

The following subsections provide the remedial action objectives for soil associated with the LAOCB unit.

Basin Soil. Based on review of ARARs and RGOs, there are two remedial action objectives for the soil at the LAOCB as follows:

1. reduce risks to human health and the environment associated with

a) external exposure to radiological contaminants

b) inhalation of radiological contaminants

c) incidental ingestion of radiological contaminants in the surface soil at the unit

d) to prevent or mitigate the release of COCs to groundwater at the unit

2. meet concentration based RGOs 
RGOs can only be achieved through off-unit removal/disposal general response actions. Therefore, technologies under the general response actions of containment and/or treatment do not achieve RGOs. While these technologies will not meet the RGOs, the goal of containment and treatment technologies are to use engineering controls and treatment to reduce risk by creating a reduction in mobility and exposure. These technologies meet the remedial action objective of reducing risk to human health and the environment and may be retained for analysis in Section 3.0.

As stated in Section 1.3.4.1, the total radionuclide activity within the LAOCB is approximately $4.2 \mathrm{Ci}$. Linear regression analysis of the maximum concentrations of gross alpha and gross beta indicates that both gross alpha and gross nonvolatile beta activity will decrease to background activities of $5.4 \mathrm{pCi} / \mathrm{g}$ and $5.09 \mathrm{pCi} / \mathrm{g}$, respectively, within $0.6 \mathrm{~m}(2 \mathrm{ft})$ from the top of the sludge (Figures 1-5 and 1-6). These activities are well below the radionuclide screening levels established for SRS of $20 \mathrm{pCi} / \mathrm{g}$ for gross alpha activity and $50 \mathrm{pCi} / \mathrm{g}$ for gross beta-gamma (WSRC, 1995a; 1996a). Considering that the RGOs (see Table 2-2) for the anthropogenic radionuclides are generally below background (from world-wide weapons fallout) or below detectable levels, the proposed remediation goal for the radionuclides in the soil of the LAOCB is to achieve the background gross alpha and gross beta activity. RGOs for nonradioactive metals (including $\mathrm{Cr}, \mathrm{Cd}$, and $\mathrm{Pb}$ ) are concentration goals to reduce risk within the acceptable EPA risk range (1E-04 to 1E-06) or below an $\mathrm{HI}$ of one (see Table 2-2).

Soil Adjacent to the Basin. No estimated carcinogenic risk or noncarcinogenic hazards are associated with exposure to soil adjacent to the Basin. Therefore, remediation of the soil adjacent to the Basin is not warranted.

LAOCB Pipeline Soil. Based on review of ARARs and the results of the BRA, remediation of the soil associated with the pipelines is not warranted. The pipeline soils $\operatorname{COCs}(\mathrm{Al}, \mathrm{As} ; \mathrm{Pb}$, and 
TI) are all naturally occurring metals in SRS soils. Therefore, hazards resulting from them may not be from unit activities. Information supporting this risk management decision include:

- Aluminum is a major component of clay minerals that are abundant in SRS soils. Lead concentrations only contribute to a hazard of one when combined with the hazard from Tl. Thallium concentrations in soil from a depth of $0-1.2 \mathrm{~m}(0-4 \mathrm{ft})$ resulted in a higher hazard than $\mathrm{Tl}$ concentrations from $0-3.7 \mathrm{~m}(0-12 \mathrm{ft})$. It is believed that $\mathrm{Tl}$ is at higher concentrations in the shallower soil possibly due to atmospheric fall-out from coalburning and smelting (where thallium is a trace component), and/or from the use of pesticides on the soil during farming before SRS was constructed. Thallium and its compounds were utilized as rodenticides and insecticides prior to 1972 (HSDB, 1994).

- $\quad$ Although As is a COC, its concentrations in SRS soils are believed to be ubiquitous throughout the site from naturally occurring sources and past farming activities. Common pesticides used on crops that were farmed on land that is now the SRS included lead arsenate, lead arsenite, and copper acetoarsenite. These pesticides could have also contributed to concentrations of $\mathrm{Pb}$ at the Basin.

\subsubsection{Remedial Action Objectives for LAOCB Pipelines}

Radioactive contamination of the internal surface of the LAOCB pipeline has been documented to be approximately 300,000 disintegrations per minute (dpm). However, specific radionuclides on the interior of the LAOCB pipelines were not speciated as part of the RFI/RI. Therefore, a BRA was not conducted for the pipelines and RGOs could not be determined. No chemicalspecific ARARs such as UMTRCA, 40 CFR 192 Subparts A through E (applies to soils) have been identified that would apply to the LAOCB pipelines. However, DOE Order 5400.5 Radiation Protection of the Public and the Environment is to-be-considered information and may apply to the LAOCB pipelines. Although the observed radiological contamination on the 
internal surfaces does not currently represent risk to human health and the environment, future deterioration of the steel walls of the pipeline could potentially release contaminants to the environment and result in unacceptable risk. Therefore, the primary remedial action objective, based on known information, is to prevent the migration of contaminants associated with the pipelines to groundwater.

\subsubsection{Remedial Action Objectives for Vegetation}

Detected radionuclides which appear to have the potential to pose ecological risk based on plant tissue residue levels and toxicity characteristics are ${ }^{137} \mathrm{Cs}$ and ${ }^{60} \mathrm{Co}$. Both of these radionuclides were detected in vegetation from the LAOCB at concentrations which are elevated above reference levels and probably unit-related. These radionuclides are typically bioaccumulated by organisms (particularly ${ }^{137} \mathrm{Cs}$ ) and have a significant potential to produce toxic effects as a result of their radioactive decay. Therefore, ${ }^{137} \mathrm{Cs}$ and ${ }^{60} \mathrm{Co}$ have the potential to pose risk to ecological receptors exposed to contaminated media directly or through the food chain, such as animals which consume either contaminated vegetation or other animals with bioaccumulated residues of these radionuclides in their tissues.

Vegetation in the immediate vicinity of the LAOCB was removed in 1993 prior to implementation of the RFI/RI. Remedial action objectives for the vegetation remaining in the LAOCB will be to prevent the uncontrolled exposure of receptors to the radionuclides contained in the remaining vegetation.

\subsection{General Response Actions}

General response actions are medium-specific actions which will lead to the accomplishment of remedial action objectives. General response actions may include actions such as institutional control, containment, extraction, treatment, or a combination of these and other general actions. 


\subsubsection{General Response Actions for Soil}

General response actions for soil associated with the LAOCB are developed based on unitspecific conditions and to satisfy the NCP (55 FR 8666). General response actions for soil adjacent to the Basin and for soil associated with the pipelines are not required. Vegetation in the vicinity of the Basin has been removed. The general response actions for the LAOCB sludge and subsoil are:

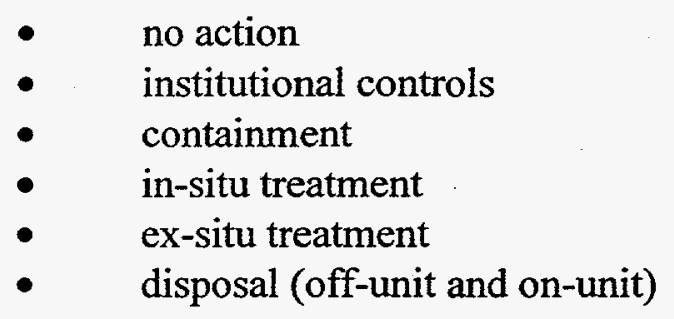

\subsubsection{Estimated Volume of Impacted Soil}

For purposes of this CMS/FS, a range of volumes of soil requiring remediation at the LAOCB are developed based on (1) the primary $\operatorname{COCs}\left({ }^{60} \mathrm{Co}\right.$ and $\left.{ }^{137} \mathrm{Cs}\right),(2)$ the level of activity in the Basin sludge with respect to depth, and (3) the lateral and vertical extent of contamination.

The concentrations of radionuclides in the LAOCB sludge and subsoil tend to decrease rapidly with depth. An analysis of the attenuation of gross nonvolatile beta and alpha emissions indicate that radionuclide concentrations (other than ${ }^{3} \mathrm{H}$ ) should reach background activity levels within $0.6 \mathrm{~m}(2 \mathrm{ft})$ from the top of the sludge. The radionuclide concentrations are also highly correlated (as expected) with the gross alpha and gross nonvolatile beta values for the samples. Linear regression analysis of the gross alpha values versus sample depth demonstrates that the sludge gross alpha values will decrease to background levels at a depth of $0.5 \mathrm{~m}(1.5 \mathrm{ft})$ from the top of the sludge (Figure 1-5). Similarly, linear regression analysis of the gross nonvolatile beta 
values versus sample depth demonstrates that the sludge gross non-volatile beta values will decrease to below detectable levels at a depth of $0.55 \mathrm{~m}(1.75 \mathrm{ft})$ from the top of the sludge (Figure 1-6).

Based on the Basin dimensions of $49 \mathrm{~m}$ long (158 ft) by $26 \mathrm{~m}$ wide $(84 \mathrm{ft})$ inside the berm, the approximate area of the LAOCB unit is $1,270 \mathrm{~m}^{2}\left(13,272 \mathrm{ft}^{2} ; 0.31 \mathrm{ac}\right)$. Since the level of activity decreases to background activities within $0.5 \mathrm{~m}(1.75 \mathrm{ft})$, but conservatively at a depth of $0.6 \mathrm{~m}$ $(2 \mathrm{ft})$, the volume of contaminated sludge/subsoil requiring remediation is estimated to be approximately $760 \mathrm{~m}^{3}\left(1,000 \mathrm{yd}^{3} ; 27,000 \mathrm{ft}^{3}\right)$.

\subsubsection{General Response Actions for Pipelines}

General response actions for the LAOCB pipelines are developed based on unit-specific conditions and to satisfy the NCP (55 FR 8666). The general response actions for the LAOCB pipelines are:

- no action

- institutional controls

- containment

- in-situ treatment

- disposal (off-unit and on-unit)

\subsubsection{Estimated Volume of Impacted Pipeline}

Based on review of ARARs and the results of the BRA, remediation of the soil associated with the pipelines is not warranted. However, the interior of the pipelines are suspected to contain radionuclide contamination similar to that found in the LAOCB soils. Therefore, only the pipelines are being considered for remediation. Two pipelines extend from the Hot Shop to the LAOCB. One pipeline is approximately $139 \mathrm{~m}(456 \mathrm{ft})$ long and has a diameter of $15 \mathrm{~cm}(6 \mathrm{in}$.) with a total surface area of $131 \mathrm{~m}^{2}\left(1,433 \mathrm{ft}^{2}\right)$. The other is approximately $131 \mathrm{~m}(431 \mathrm{ft})$ long 
and has a diameter of $15 \mathrm{~cm}\left(6 \mathrm{in}\right.$.) with a total surface area of $123 \mathrm{~m}^{2}\left(1,354 \mathrm{ft}^{2}\right)$. The interior of the pipelines are assumed to be empty; however, volumes of the pipelines are $10 \mathrm{~m}^{3}\left(353 \mathrm{ft}^{3}\right)$ and $9.2 \mathrm{~m}^{3}\left(324 \mathrm{ft}^{3}\right)$, totaling $19.2 \mathrm{~m}^{3}\left(677 \mathrm{ft}^{3}\right)$. The pipelines are constructed of cast iron.

\section{$2.4 \quad$ Listing of Technologies}

The purpose of this section is to establish a preliminary list of treatment technologies that are potentially applicable to LAOCB soils and pipelines based on the remedial action objectives and the general response actions. A source of information for the listing of remedial technologies is from the Remediation Technology Roundtable, conducted on January 17 and 18, 1995, which was assembled to address contamination associated with basins at SRS. The Remediation Technology Roundtable consisted of a panel of technical experts assembled to initiate critical, objective dialogue concerning potentially feasible remedial technologies and general response actions that could be used at the LAOCB. Technical merits and limitations of each technology and general response action were discussed in the open forum. The following technologies and general response actions were identified:

- In-situ grouting

- Capping

- Barriers

- In-situ vitrification
- Removal

- Ex-situ treatment

- Storage/Disposal

In addition to the Remediation Technology Roundtable, the initial list of technologies is based on the following information:

- Superfund Innovative Technology Evaluation Program: Technology Profiles, Sixth Edition (EPA, 1994)

- $\quad$ Remediation Technologies Screening Matrix and Reference Guide (EPA, 1993a)

- Technological Approaches to the Cleanup of Radiologically Contaminated Superfund Sites (EPA, 1988d) 
- $\quad$ Approaches for the Remediation of Federal Facility Sites Contaminated with Explosives or Radioactive Wastes (EPA, 1993b)

- $\quad$ EPA - Applications Analysis Reports

- DOE Office of Environmental Management and Office of Technology Development Technology Summaries

- Hazardous Materials Control Resources Institute - HMC/Superfund Proceedings

- DOE - Environmental Remediation Conference Proceedings

- American Society of Mechanical Engineers, DOE, and EPA - Mixed Waste Proceedings

- American Nuclear Society, American Society of Mechanical Engineers, DOE, and University of Arizona - Waste Management Proceedings

- Health Physics Society - Low-Level Radioactive Waste Management Proceedings

- $\quad$ EPA Database - Alternative Treatment Technology Information Center (ATTIC)

- EPA Database - Vendor Information System for Innovative Treatment Technologies (VISITT)

- Hazardous Waste Management (LaGrega et al., 1994)

- $\quad$ Nuclear Chemical Engineering (Benedict et al., 1981)

- Radioactive Waste Management Handbook Volume 1: Near-Surface Land Disposal (Kittel, ed., 1989)

- $\quad$ Appendix D of the NCP

Additionally, personal communication with technical experts provided by TELTECH, Technical Knowledge Service, was conducted. TELTECH is a service that provides a network of experts that assist in searches for technical information and reference literature.

Tables 2-3 and 2-4 list the potentially applicable technologies for the LAOCB soil and LAOCB pipelines, respectively. These technologies are based on unit-specific conditions, the areas and volume of media potentially requiring remediation, the remedial action objectives, the general response actions, and the results of the Remediation Technology Roundtable. 


\subsection{Screening of Technologies}

The purpose of this section is to describe specific technology types, including process options, for the general responses associated with LAOCB soil and pipelines. Identified technologies will be screened using the criteria of effectiveness, implementability, and cost. Some technologies which pass this screening (i.e., are retained) may apply to only a discrete portion of a medium, but may be used in conjunction with other technologies to form an overall remediation strategy. Remedial alternatives will be formulated in Section 3.0 using technologies retained after technology screening. Remedial alternatives developed in Section 3.0 are subjected to a detailed evaluation in Section 4.0.

\subsubsection{Screening Criteria}

The NCP provides three basic criteria for screening of technologies. The NCP criteria for screening of technologies are:

- $\quad$ effectiveness

- implementability

- $\quad$ cost

CMS guidance does not specify the three NCP criteria as such; however, guidance does indicate that the screening process should eliminate corrective measures that may prove infeasible to implement, that rely on technologies unlikely to perform satisfactorily or reliably, or that do not achieve the remedial action objectives within a reasonable time period.

\subsubsection{Effectiveness}

For a technology to be effective, it must achieve specified objectives, compatible with the contaminant characteristics and unit conditions, and must be protective of public health and the 
environment. To accomplish this, the technology itself must be effective in reducing or eliminating any short-term and long-term risk to human health or the environment directly associated with the waste unit and must not pose adverse impacts on the environment, public health, or public welfare. Technologies for which unit contaminants or conditions clearly limit their effectiveness or which do not provide adequate protection of the environment, public health, and public welfare will be rejected (i.e., not retained) and will not be considered for detailed analysis. Additionally, technologies which have not demonstrated effectiveness at similar units will be eliminated from further consideration.

\subsubsection{Implementability}

Implementability addresses both the technical and institutional feasibility of applying a technology. Under this criterion, technologies are evaluated based on the technical feasibility, availability of resources and equipment, and the administrative feasibility of implementing them. The nature of the technology should be such that it can be implemented in a cost effective and timely manner in the physical setting associated with the LAOCB. In addition, the implementation of the technology should not elicit substantial public concerns in the community. Site accessibility, available area, and potential future use of the property may also affect the implementation of certain technologies. Mobilization and permitting requirements, where. applicable, must be workable. Preliminary consideration is also given to regulatory constraints such as handling, disposal, and treatment requirements that would effect the implementation of certain remedial technologies. These considerations will be evaluated further during detailed analysis (Section 4.0) for retained technologies when action-specific ARARs are developed. Technologies that are not technically or administratively feasible will be removed from further consideration. 


\subsubsection{3 $\underline{\text { Cost }}$}

A qualitative cost evaluation is provided so that cost comparisons can be made among technologies. Technology costs are described as being high, medium, or low, relative to technologies of similar type (e.g., containment technologies). Qualitative evaluations take into consideration capital costs and operation and maintenance costs, and are based upon prior estimates, previous experience, and engineering judgment. The costs are not intended for budgetary purposes. Those technologies that demonstrate comparable levels of applicability, effectiveness, and implementability as other technologies but at significantly greater cost will be eliminated. Similarly, technologies that are equivalent in cost but are clearly less effective than other retained technologies will also be rejected. Otherwise, cost will not be used as a criterion to screen technologies at this point in the CMS/FS process.

\subsubsection{Description and Evaluation of LAOCB Soil and LAOCB Pipeline Technologies}

The following subsections present an evaluation of the individual remediation technologies based on the three screening criteria discussed above. Technologies retained after this screening will be used to develop comprehensive remedial alternatives in Section 3.0.

\subsubsection{No Action}

No Action is not a technology but is a general response action that relies on natural attenuation mechanisms to reduce contaminant concentration levels to below proposed concentration-based remediation goals. Under this general response, no efforts would be taken to monitor, remove, treat, or otherwise mitigate the potential spread of contaminants at the LAOCB or the associated pipelines. In addition, no actions would be taken to minimize the potential for human exposure to contaminants. For these reasons, no action is not an effective response for the LAOCB soils or the LAOCB pipeline. 
The No Action response is readily implemented and represents the least expensive (baseline) alternative possible. Costs associated with the No Action alternative would include a review of remedy every five years for 30 years as required under SARA. The NCP requires that the No Action alternative be retained throughout the CMS/FS as a basis for comparison during the detailed analysis of alternatives.

\subsubsection{Institutional Controls}

Institutional controls are administrative measures taken to minimize the potential for human exposure to contamination. The institutional control alternatives include institutional property restrictions to include fencing/posting, security access control, and deed restrictions. Institutional controls are potentially effective and relatively easy to implement. In addition, costs associated with this alternative are considered low relative to other remedial responses. Costs would include continued maintenance of existing institutional controls and possibly additional controls as warranted.

\section{$\underline{\text { LAOCB Soil }}$}

Some institutional controls already exist at the LAOCB such as the chainlink fence surrounding. LAOCB, posting of warning signs that describe hazards associated with the unit, periodic groundwater monitoring, and SRS security measures. An additional institutional control measure to further minimize the potential for future human exposure is deed restrictions. Deed restrictions would inform hypothetical developers or buyers of affected properties of previous waste disposal activities, and would limit the types of future activities that could be conducted on the property (e.g., restrictions on excavating and groundwater use). Institutional controls for the LAOCB soil will not be considered as a stand-alone remedy, but will be considered in conjunction with other remedies that leave LAOCB contaminants in place. 


\section{$\underline{\text { LAOCB Pipelines }}$}

Institutional controls associated with the pipeline would include maintenance of the current controls including monitoring primary road entrances, and the exclusion fence around the SRS facility. Additional control measures, include fencing and posting warning signs around the pipelines, and deed restrictions. Institutional controls will be considered as a stand-alone remedy for the LAOCB pipelines.

\subsubsection{Containment}

Containment technologies involve the construction of an engineered barrier to isolate wastes. When properly constructed and maintained, containment technologies can provide a reliable and effective method for controlling direct exposure to waste and minimizing contaminant transport through leaching. Two containment technologies, capping and vertical barriers, are considered for application at the LAOCB. Capping is the only containment technology considered for application at the LAOCB pipelines.

Capping. Capping is a method commonly used to contain waste by covering the waste with a protective barrier to prevent infiltration that could potentially mobilize contaminants that may otherwise contaminate underlying groundwater and to deter access to the waste. For application at radioactive or mixed waste sites, the cap may also be designed to attenuate gamma radiation associated with all radionuclides present. Attributes of a properly designed cap include (1) resistance to water and wind erosion, (2) stability against subsidence, cracking and slope failure, and (3) protection from freezing and intrusion by plants and animals.

Materials used to construct a cap may include topsoil, clay, gravel, geosynthetics, asphalt, and concrete. RCRA caps are multilayer covers used to contain and protect RCRA units having waste left in place after closure, but have also been used to contain low-level radioactive and mixed wastes. Although designs may vary, a typical RCRA cover consists of a vegetative 
surface cover supported by a soil layer, followed by a drainage layer to promote lateral flow away from the waste unit, and two low permeability layers consisting of a geosynthetic material and a compacted clay layer, respectively. Other types of caps, such as single layer earthen cap, asphalt cap, and capillary barrier cap designs, have been constructed at numerous waste disposal sites, and function similarly to RCRA caps; however, RCRA cap designs are engineered to maximize effectiveness (e.g., multilayer barrier, drainage layer), are readily accepted by the regulatory community, and have been demonstrated to be more effective than innovative capillary designs (Warren et al., 1994). Other cap designs including single layer earthen caps and asphalt caps are generally less expensive to construct than a RCRA cap designs but usually are not designed with redundant safety features and are not as readily accepted by regulatory agencies for containing low-level radioactive or mixed waste.

Capping will be retained for further consideration since it is a mature technology and has been demonstrated effective at many hazardous waste sites and has been used for remediating radiologically contaminated sites. Furthermore, cap construction is easily implemented and associated costs are relatively low. RCRA cap designs have been demonstrated effective at similar sites and would be the only cap design considered should capping be considered as a stand-alone remedy at the LAOCB and the LAOCB pipelines. Otherwise, all other cap designs evaluated may be considered for application at the LAOCB and the LAOCB pipelines as long as they are used in conjunction with other remedial technologies (e.g., stabilization, vitrification). Should capping be conducted at the LAOCB and the LAOCB pipelines, selection of cap design should consider alternative-specific remediation goals and should therefore be deferred until detailed design.

Vertical Barriers. When properly constructed, vertical barrier technology can be used to isolate or encapsulate areas of contamination and greatly restrict the mobility of contaminants. Three conventional vertical barrier technologies, slurry cut-off wall, grout curtain, and sheet pile wall, and one innovative vertical barrier technology, cryogenic barriers, are discussed below. 
Slurry cut-off walls are among the most common subsurface barriers because they can vastly reduce groundwater flow in unconsolidated earth and are readily constructed. Slurry walls are almost always used in conjunction with other means of containment (e.g., cap) or treatment. When combined with groundwater extraction systems, they provide a means of establishing an inward hydraulic gradient, further reducing contaminant mobility beyond the cut-off wall. Generally, they are constructed in vertical trenches that are excavated under a slurry (e.g., bentonite/water). Materials used to backfill the slurry trench may include soil/bentonite or cement/bentonite mixtures. Treatability testing of construction materials under unit-specific conditions should be considered. Alternative materials and installation methods that could be considered with this technology are also available.

Grout curtains are subsurface low-permeability barriers constructed using pressure injection. The grout is pressure injected through a pipe and into the matrix to be treated. The process is repeated with overlapping injections until the wall is completed. As with slurry cut-off walls, grout curtains can be constructed with a variety of materials, and treatability testing of construction materials should be considered. Similarly, construction of the grout curtain should be pilot-tested under unit-specific conditions to determine effectiveness. Unlike slurry wall construction, depth is generally not a limiting factor in grout curtain construction.

Sheet pile walls are constructed using pre-formed sheet pilings that are driven into the ground and connected by interlocking joints. Sheet pilings can be constructed of various materials including pre-cast concrete and steel. A limitation of sheet pile wall construction is interferences from rocky soil that can deflect the piles as they are being driven; however, this should not be a problem at the LAOCB. When first placed into the ground, the sheet pile wall is relatively permeable because the interlocks are necessarily loose to facilitate placement. Over time, fine soil particles fill the void and form a seal. The time required for the voids to seal will depend upon the rate of groundwater flow and the texture of host soil (EPA, 1985). 
Cryogenic barriers are used to isolate a contaminated zone from a non-contaminated zone. Cryogenic barriers can also be used to contain contamination in a frozen zone, thereby immobilizing it. The process freezes soil in-place by circulating liquid nitrogen or other refrigerant material through the soil or sediment. Continuous application of refrigerant is required to maintain the zone in the frozen state. This technology has been used in the mining industry, but has not yet been demonstrated for environmental restoration purposes. This technology has been included in the DOE Buried Waste Integrated Demonstration, but as yet no data is available on: (1) the degree of confinement of contaminants either in the ice zone; or (2) the migration of contamination across the ice zone. Based on the lack of performance data, this technology is eliminated from consideration for application to the LAOCB.

Of the three conventional vertical barriers being considered for application at the LAOCB, the most effective would be the slurry cut-off wall. If properly constructed, the slurry cut-off wall would be less permeable than the grout curtain or the sheet pile designs. Furthermore, assessment of the effectiveness and reliability of construction of grout curtain and sheet pile wall designs would be much more difficult to assess than it would be for slurry cut-off wall design. Additionally, the slurry cut-off wall could be keyed into the hard-pan that exists under the Basin. Construction of a capping system over the Basin would result in totally enveloping the waste in the Basin thereby immobilizing the contaminants.

Total costs associated with the construction of vertical barriers would be approximately the same for each of the three designs considered. Because of the time required for construction, the grout curtain design would cost more than the slurry cut-off wall and sheet pile designs. Based on effectiveness and cost, only slurry cut-off walls will be retained for further consideration. 


\subsubsection{In-Situ Treatment}

In-situ treatment technologies treat soil in place. For soil containing radioactive and nonradioactive inorganic constituents, in-situ treatment technologies generally involve a chemical and/or physical treatment process that immobilizes the contaminants. One exception is electrokinetic processing technology which reduces contaminant concentrations in soil. Relative to comparable ex-situ treatment technologies, in-situ remedial technologies have the advantages of minimal handling of contaminated media, lower capital costs, and minimizing exposure to remedial workers. In-situ treatment technologies being considered for LAOCB soil are stabilization/solidification, vitrification, and electrokinetic soil processing. The only in-situ treatment technology considered for the LAOCB pipelines is stabilization/solidification.

One limitation that is common to most in-situ treatment technologies is that there is limited opportunity to verify treatment effectiveness or monitor process quality control in-situ. For example, there is the potential that the entire soil matrix may not be sufficiently treated at the conclusion of the $\mathrm{S} / \mathrm{S}$ process. Consequently, in-situ $\mathrm{S} / \mathrm{S}$ technology may be a potentially effective technology for treating LAOCB soil and the pipelines as long as it is used in conjunction with other remedial technologies (e.g., capping, institutional controls, excavation and disposal). In-situ S/S could also be considered to pretreat contaminated soil and minimize short-term exposure to remedial workers prior to ex-situ treatment or off-unit disposal.

In-Situ Stabilization/Solidification (S/S). The terms "stabilization" and "solidification" have often been used interchangeably, and with other terms such as "chemical fixation" and "immobilization". Stabilization refers to those technologies that reduce the hazard potential of a waste by converting the contaminants into their least soluble, mobile, or toxic waste form without regard to the physical nature or handling characteristics, whereas, solidification refers to technologies that encapsulate the waste in a monolithic solid of high structural integrity. 
Solidification does not necessarily involve a chemical interaction between the wastes and the solidifying reagents, but may mechanically bind the waste into the monolith. When solidified, contaminant migration is restricted by reducing the surface area exposed to leaching, and/or by isolating the waste within an impervious capsule. Encapsulation may be one of fine waste particles (microencapsulation) or of a large body or container of waste (macroencapsulation) (Conner, 1994). Since there is often potential for stabilization and solidification to occur in a single process, stabilization/solidification technologies will be referred to as $\mathrm{S} / \mathrm{S}$ technologies hereafter.

Most S/S technologies are relatively simple to implement, involving standard stabilizing or solidifying agents and mechanical equipment. Stabilizing or solidifying agents may include, lime/fly ash pozzolans, Portland cement, silicate, apatite, thermoplastics, or derivatives of these and/or other reagents. A treatability study has been conducted to determine appropriate $\mathrm{S} / \mathrm{S}$ reagents for contaminants associated with the LAOCB soil. Several reagents were evaluated primarily on the basis of mixability, strength, and leaching of radioisotopes from stabilized/solidified mixtures. Results of the study indicate that conventional reagents (Portland cement, bentonite, and silicate) can be used to effectively treat LAOCB soils such that they meet EPA and DOE standards (WSRC, 1996c).

S/S technologies may be applied in-situ or ex-situ. In-situ S/S technologies are conducted leaving the waste in-place without the benefit of excavation from, or treatment outside of, the unit. Reasons for stabilizing or solidifying a waste in-situ may include minimizing waste handling and exposure to remedial workers during treatment and minimizing the potential for contaminant leaching should the waste be left in place; eliminating the need for off-unit transport and/or disposal of the waste; and improving waste handling characteristics should the waste be transported off-unit for further treatment and/or disposal. A description of ex-situ S/S technology and its potential for application at the LAOCB is presented in Section 2.6.2.5. 


\section{LAOCB Soil}

In-situ $\mathrm{S} / \mathrm{S}$ is accomplished by mixing the $\mathrm{S} / \mathrm{S}$ reagent into the waste by some mechanical means, such as a backhoe, tiller, auger, or injection/jetting technique. The most common device used for mixing S/S reagent into relatively shallow soil is the backhoe because it is widely available and is suitable for many applications; however, intimate mixing is difficult to achieve using conventional backhoes and they are limited by their reach. Tractor mounted rotary tillers can achieve more intimate mixing than can the backhoe, but they are limited to applications at depths of $1.5 \mathrm{~m}(5 \mathrm{ft})$ or less.

For deep soil grouting, vertical augering and injection/jet grouting can provide intimate mixing or contact with the $\mathrm{S} / \mathrm{S}$ reagent, respectively, and are less restricted by depth than most in-situ $\mathrm{S} / \mathrm{S}$ reagent delivery techniques. Vertical augering involves drilling augers equipped with cutting and hollow mixing blades. A conceptual crane mounted soil/grout mixing system is illustrated on Figure 2-2.

In-situ grout injection involves the injection of grout into the waste matrix via an injection pipe. The pipe is either drilled or hammered into the waste matrix and the grout is injected under pressure into voids within the waste matrix via the injection pipe. Once all of the voids are filled at a particular depth, the pipe is raised and more grout is injected from the bottom of the pipe. The injection process continues until the surface is reached. Grout injection has been conducted at depths of $18.3 \mathrm{~m}(60 \mathrm{ft})(\mathrm{EPA}, 1993 \mathrm{~b})$. A conceptual track mounted jet-grout drilling system is illustrated on Figure 2-2.

Should in-situ stabilization be conducted at the LAOCB, the type of S/S reagent delivery system used may depend primarily upon the type of S/S reagent and recipe that is to be used since the reagent must be amenable to the reagent delivery system. An advantage of the auger soil mixing system is that it is capable of utilizing a wider range of $\mathrm{S} / \mathrm{S}$ reagent recipes than can injection or jet grouting techniques. 
The primary disadvantage of the auger soil mixing systems over injection or jet grouting systems is that the auger soil mixing systems typically generate more air emissions than do injection or jet grouting systems, or require an off-gas collection and treatment system. Also, there is generally an increase in treated soil volumes associated with auger soil mixing systems, whereas injection or jet grouting systems produce little or no volume increase.

In-situ $\mathrm{S} / \mathrm{S}$ is a potentially cost effective technology for treating contaminated soil at the LAOCB. S/S reagents have been identified through a treatability study and indicates that the mobility of LAOCB contaminants could be significantly reduced over current conditions (WSRC, 1996c). Should in-situ S/S technology be used at the LAOCB, selection of the type S/S reagent and $\mathrm{S} / \mathrm{S}$ reagent delivery system would be based upon treatability study results and $\mathrm{S} / \mathrm{S}$ treatment objectives.

Although specialized equipment and extensive site preparation may be required, in-situ S/S technology should be reasonably implementable and cost effective. Costs associated with in-situ $\mathrm{S} / \mathrm{S}$ are considered medium since they should be less than the costs associated with other in-situ technologies.

\section{$\underline{\text { LAOCB Pipelines }}$}

In-situ $S / S$ of the LAOCB pipelines would include filling the pipelines with an appropriate $S / S$ reagent (Portland cement/bentonite grout) from the L-Area Hot Shop (LAHS) end of the pipelines to the LAOCB. Grouting would immobilize residual radionuclide contaminants within each pipeline. Grouting the pipelines would not require specialized equipment or extensive site preparation, therefore this technology should be readily implementable and cost effective. Before initiating S/S technologies, one technology, High Pressure Wash, may be performed to "washout" residual contaminants within the pipelines into the LAOCB. Once discharged to the LAOCB, the washwater could evaporate and the radionuclides would remain in the basin for 
potential future remediation. High Pressure Wash will be developed as an alternative in Section 3.0 .

Following in-situ $\mathrm{S} / \mathrm{S}$, the pipelines may either remain in place or be excavated and removed for either on-unit (inside the LAOCB) or off unit disposal (off-unit disposal technologies are presented in Section 2.5.2.6). If the grouted pipelines are excavated and disposed of inside the LAOCB, further S/S technologies would be implemented inside the LAOCB to further encapsulate the outside of the pipelines, and create a monolith that would further reduce the mobility of the pipeline contaminants significantly. Excavation and disposal techniques may require specialized waste and material handling equipment to reduce contaminant mobility of airborne particulate emissions from the pipelines and potentially contaminated soil on the outside of the removed pipes.

In-Situ Vitrification. Vitrification is a process that is used to convert materials into a glass or glassy substance, typically through a thermal process. Although some vitrification processes do not require heat (e.g., vapor deposition, solution hydrolysis), only thermal vitrification will be considered for application at the LAOCB. Thermal vitrification is conducted at temperatures ranging from $1,100^{\circ} \mathrm{C}$ to $2,000^{\circ} \mathrm{C}\left(2,012^{\circ} \mathrm{F}\right.$ to $\left.3,632^{\circ} \mathrm{F}\right)$. At these temperatures, organic constituents that have not volatized from the source are destroyed via pyrolysis or combustion, whereas nonvolatile inorganic constituents are stabilized within the glass matrix. Stabilization is accomplished by either incorporating nonvolatile inorganics into the glass structure or by encapsulating them in the glass product. In the case of radionuclides, these immobilization processes may also prevent some radioactive decay products from escaping into the environment (EPA, 1992). Volatized organics and inorganic constituents are captured and processed in an off-gas treatment system. Organic contaminants are not a significant concern at the LAOCB; therefore, application of vitrification technology for the removal or destruction of organic compounds will not be addressed unless their presence influences vitrification effectiveness or efficiency. 
The primary advantage of vitrification is that the final waste product is very durable and performs well in leach tests (i.e., TCLP testing). Another advantage of vitrification is that it can incorporate a wide variety of contaminants and accompanying feed materials without a significant decrease in performance of the final product. Vitrification may also reduce the final volume of a waste material. Vitrification, however, is energy intensive and may therefore be more expensive that other remedial technologies. Another limitation of vitrification is that volatile organic and inorganic constituents may be driven from the waste during processing. For in-situ vitrification, volatilization and other processes may cause contaminants to migrate from the source into the surrounding soil.

Waste unit characteristics and treatment objectives play an important role in determining whether in-situ vitrification is suitable for a particular application. Moisture content of the process material will affect the efficiency of the vitrification process. Any material with a high moisture content will increase the processing time and energy demands by first requiring that the water be driven off. Also, it is possible that at greater than five percent free water within the matrix, the water may react vigorously with the melt as it vaporizes. The presence of standing water in the LAOCB would reduce vitrification efficiency unless the water is first removed prior to processing. This would require that the water be properly characterized, stored and/or treated by other treatment processes. Soil composition will affect product characteristics such as density and chemical resistance, and will influence processing parameters such as thermal conductivity, fusion temperature, specific heat, electrical conductivity, and melt viscosity. The primary soil characteristics limiting in-situ vitrification are high quartz content and low alkali flux content (EPA, 1992). Soil are typically amenable to vitrification since they often contain a sufficient concentration of silica $\left(\mathrm{SiO}_{2}\right)$ and alumina $\left(\mathrm{Al}_{2} \mathrm{O}_{3}\right)$ which form the skeleton of the amorphous glass product. In a recent bench-scale study, samples of SRS soil proved difficult to vitrify because of soil composition. Subsequently, two bench-scale flux enhancement techniques were conducted on the SRS soil. The first test involved the placement of a starter layer (sand) over the 
SRS soil. This resulted in the melt progressing outward without achieving a significant penetration into the SRS soil. The second test involved pre-mixing of alkali materials into the soil. Although this test was highly successful, the need for pre-mixing of the soil diminished the many advantages of the process of vitrifying the contaminants without pretreatment (EPA, 1992). Pre-mixing of the LAOCB soil could be achieved relatively easily. In-situ vitrification will be retained for further consideration.

In-Situ Electrokinetic Soil Processing. Electrokinetic soil processing decontaminates soil media by influencing the negative charge that is present on almost all soil surfaces. The negatively charged soil surface attracts positively charged cationic species including inorganic contaminants. The degree to which a soil is capable of attracting cationic species is referred to as the soil cation exchange capacity.

Electrokinetic soil processing involves the insertion of anode and cathode electrodes into contaminated soil. A conditioning fluid is circulated at the electrodes and an electric current is applied to produce an electric potential between the anode and cathode. The conditioning fluid serves as a conducting medium and a means to extract and exchange charged species that collect at the electrodes. This process is called electrophoresis which is the mass flux of charged (ionic) particles under an electric field. Electrophoresis is at least an order of magnitude faster than transport of species by diffusion or electroosmotic advection (EPA, 1993b).

During electrokinetic soil processing, some of the contaminant species electrodeposit on the electrodes, whereas others are extracted through the use of chemical processes or ion exchange systems within the process control container. The conditioning fluid is used to control and/or depolarize the cathode reaction so that the base generated does not lead to premature precipitation of the incoming species. Conditioning fluids being investigated include acetic acid and chelating agents. Acetic acid depolarizes the reaction at the cathode and prevents base 
formation. The acetate anions migrate into the soil and solubilize contaminant species. Chelating agents are used to solubilize specific contaminants (EPA, 1993b).

Electrokinetic soil processing can be relatively inexpensive when compared to other in-situ treatment technologies for mixed waste. Pilot-scale studies indicate that energy costs associated with electrokinetic soil processing are about $\$ 15 / \mathrm{m}^{3}\left(\$ 0.42 / \mathrm{ft}^{3}\right)$ of soil over a three month period (EPA, 1993b). Electrokinetic soil processing costs are therefore considered low.

Electrokinetic soil processing can be used to treat soil for the removal of lead, cadmium, uranium, thorium, radium and polar ionic species (EPA, 1993b). Electrokinetic processing may effectively treat soil containing other contaminants (e.g., Cs); however, documentation of this is limited. Application of electrokinetic soil processing has been limited to bench-scale and pilotscale testing. Although testing results have demonstrated varying degrees of success, this technology has not been demonstrated under full-scale applications. Furthermore, electrokinetics may not effectively treat all LAOCB COCs. Consequently, electrokinetic soil processing would have to be conducted in conjunction with other remedial actions to be effective. On the basis of implementability and effectiveness, electrokinetic soil processing will not be retained for further consideration in this report.

\subsubsection{Ex-Situ Treatment}

Ex-situ treatment involves the excavation and subsequent treatment of soil. The treated soil are either returned to the unit or taken off-unit for final disposal. Relative to comparable in-situ treatment technologies, ex-situ remedial technologies have the advantages of being able to verify the effectiveness of the treatment, verify that all contaminated media has been treated, and, in the case of off-unit disposal, remove all soil-borne contamination from the unit. Ex-situ treatment technologies being considered for LAOCB soil are stabilization/solidification, vitrification, 
chemical extraction, and physical separation. No ex-situ treatment technologies are being considered for the LAOCB pipelines.

Stabilization/Solidification (S/S). Stabilization/solidification technology is explained in detail in Section 2.6.2.4 under in-situ stabilization/solidification. Following is an evaluation of ex-situ S/S technology as it would apply at the LAOCB.

Ex-situ S/S involves the controlled mixing of waste materials with S/S reagents outside of the waste source area. The sludge and subsoil may require some form of in-situ pretreatment prior to excavation in order to minimize waste handling during ex-situ S/S. Treated waste may undergo further treatment prior to being returned to the (former) source area or being transported to an off-unit location for final disposal. Equipment typically used for ex-situ S/S applications includes reagent storage tanks, feed pumps, metering device, mixing equipment, and waste handling equipment.

Because the $\mathrm{S} / \mathrm{S}$ treatment process is conducted ex-situ, a broad selection of $\mathrm{S} / \mathrm{S}$ reagents is available to treat the waste. $S / S$ reagents that have been successfully used or are potentially effective for the treatment of low level radioactive or mixed wastes include Portland cementbased systems, pozzolanic systems, organic polymer thermoplastic systems, and sulphate polymer thermoplastic systems.

Macroencapsulation systems generally involve the use of drums, jackets, containers, or vaults to physically contain waste materials. The containers may be constructed of steel (i.e., drums), concrete (i.e., vaults), or other material depending upon waste characteristics and method of disposal. Because macroencapsulation is labor intensive, this technology is usually applied to limited volumes of either very toxic or very mobile wastes. Furthermore, waste storage facilities may have limited capacity. Because the volume of LAOCB wastes is relative large, and the 
waste can be effectively treated using more conventional S/S technologies, macroencapsulation will not be retained for further consideration.

Ex-situ S/S is a potentially cost effective technology for treating contaminated soil at the LAOCB. Treated soil may be placed back into the LAOCB or disposed at an off-unit disposal facility. If treated soil are returned to the LAOCB, the mobility of LAOCB contaminants should be significantly reduced over current conditions. Similarly, treated soil that are disposed of at an off-unit facility should exhibit improved handling characteristics (e.g., reduced contaminant mobility, minimal problems associated with airborne particulate emissions). The cured waste form will require testing to verify that it meets EPA and/or DOE disposal standards (England et al., 1991). A treatability study has been conducted and indicates that standards cañ be met using conventional reagents and equipment (WSRC, 1996c).

Ex-situ S/S may require extensive waste handling prior to excavation and S/S. Specialized waste and material handling equipment may be required, depending upon the $S / S$ reagent selected to treat LAOCB soil; however, required equipment should be available. Otherwise, ex-situ S/S technology should be reasonably implementable. Extensive site preparation (e.g., clearing, construction) would likely be required, but efforts to prepare the unit should not interfere with implementation of this technology. Costs associated with ex-situ S/S are considered low relative. to other ex-situ treatment technologies.

Ex-situ S/S of mixed waste soil is a demonstrated technology for treating low level radioactive and mixed waste soil. Therefore, ex-situ S/S will be retained for further consideration as a treatment or pretreatment option for LAOCB wastes. Should ex-situ S/S technology be used at the LAOCB, selection of the type S/S reagent would be based upon treatability study results and S/S treatment objectives. 
Ex-Situ Vitrification. Vitrification technology is explained in detail in Section 2.6.2.4 under insitu vitrification. An evaluation of ex-situ vitrification technology as it would apply at the LAOCB appears below.

Three types of joule heating melters that can be used for ex-situ vitrification of waste are the liquid-fed ceramic melter, stir-melter, and the Terra-Vit melter. The liquid-fed ceramic melter and the stir-melter are relatively large and stationary vitrification units typically used to vitrify high-level radioactive waste. In contrast, the Terra-Vit melter is mobile and designed for temporary, on-unit applications such as the LAOCB. Consequently, the application of ex-situ vitrification technology will focus on the Terra-Vit design.

The Terra-Vit melter is a low-cost vitrification design consisting of an earthen pit covered by a roof of high-temperature refractory materials. The roof provides access ports for the deployment of electrodes. The electrodes are positioned more than $1.8 \mathrm{~m}(6 \mathrm{ft})$ inside the pit periphery and terminate well above the bottom of the pit. A conceptual Terra-Vit melter design is illustrated in Figure 2-3.

Once constructed, the electrodes are lowered into the pit and joule heating is commenced to establish a molten floor. Wastes are then gravity fed through an influent port located between the electrodes onto the already molten material. As the waste is heated, it too becomes molten after which it is discharged from the pit periphery and is poured into waste containers for final disposal.

A 22.7 metric tons/day ( 25 ton/day) unit was reportedly constructed at the Hanford Site to treat contaminated soil. Unit construction took less than four weeks and the total project, including design, safety approvals, and procurement lasted eight weeks. Total construction costs were less than $\$ 500,000$. 
Ex-situ vitrification is a demonstrated technology and is potentially effective for remediating LAOCB soil. Vitrification is the BDAT for treating high level radioactive wastes and should therefore demonstrate comparable effectiveness on low-level radioactive waste streams. Because the process is ex-situ, soil can be amended with additives to overcome deficiencies of alkali elements in SRS soil. Treatability testing would be warranted to determine the optimum blend of amendments. Implementability of the Terra-Vit design is a concern since it would be necessary to design and construct a Terra-Vit melter for application at the LAOCB. It is projected that the treatability study, design, and construction of a Terra-Vit melter can be accomplished within one year (Tixier, 1995).

Costs associated with ex-situ vitrification treatment technology is relatively high compared to other ex-situ treatment technologies. This assessment is based upon capital costs to treat the waste and does not take into consideration costs associated with waste excavation or final disposal. Ex-situ vitrification is retained for further consideration.

Chemical Extraction. Chemical extraction is a separation technology that uses chemicals to extract contaminants from an environmental media (e.g., soil, sediment). Historically, chemical extraction technology has been used in ore benefication processes; however, the objective for its use was to remove only specific radioactive constituents from uranium mill tailings. These. chemical extraction processes include the use of water, inorganic salts, mineral acids, and complexing reagents as extraction agents. If used at the $\mathrm{LAOCB}$, one of the primary objectives of chemical extraction would be to concentrate radioactive contaminants contained in unit soil, thereby reducing the final volume of contaminated media to be treated and/or disposed.

The applicability of chemical extraction technologies is very unit-specific. Consequently, extensive treatability testing would be needed to determine whether chemical extraction technology would be potentially effective at achieving remediation goals. Information needed to apply chemical extraction technologies includes (1) physical, chemical, and mineralogical 
characteristics of the soil, (2) radionuclide concentration for each particle size fraction, and (3) water analysis for total suspended solids, $\mathrm{pH}$, hardness, and background radiation.

There are four general types of chemical extraction technologies currently being developed. These technologies are chemical extraction using water, inorganic salts, mineral acids, and complexing agents. Of these chemical extraction processes, chemical extraction using mineral acids appears to show the most promise based upon the number of radionuclides for which it is effective, and reported removal efficiencies. Reported chemical extraction rates vary significantly for different soil. In one particular study, removal efficiencies for Ra, Th, and U were reportedly as high as 97, 99, and 99, respectively; however, chemical extraction using mineral acids, the treated soil must be treated a second time to remove undesirable anions $\left(\mathrm{NO}_{3}{ }^{\circ}\right.$, Cl) (EPA, 1988d). Also, LAOCB soil contain radionuclides other than those that are amenable to chemical extraction. Extensive treatability testing would be warranted to determine which, if any, of these chemical extraction technologies could effectively treat LAOCB soil.

In addition to the process limitations mentioned above, there is limited benefit associated with technologies that reduce the concentration of radioactive contaminants in low-level radioactive waste media since the Nuclear Regulatory Commission (NRC) has instituted a moratorium on "below regulatory concern" (BRC) policy implementation. BRC is a waste classification that was proposed by the NRC in accordance with the Low-Level Radioactive Waste Policy Act that would allow for a greater number of disposal options for waste having "insignificant" levels of radioactivity. It has since been determined that authority to promulgate a BRC regulation or standard lies with "other Federal agencies". Consequently, DOE is not authorized to promulgate either a $\mathrm{BRC}$ regulation or a BRC standard, and will continue to manage low-level radioactive wastes that might meet future BRC standards and regulations until appropriate regulations are implemented (DOE, 1994b). 
On the basis of effectiveness, chemical extraction technologies will not be considered for treating LAOCB wastes.

Physical Separation. Many contaminants in soil, including radioactive contaminants, may be associated with the smallest fraction of soil particles or fines. By segregating contaminated soil based upon the physical characteristic of size, the soil contaminants may be concentrated in the fines, thus reducing the volume of soil requiring subsequent treatment or disposal. Soil may also be segregated on the basis of size as a pretreatment step to remove oversized materials (e.g., rocks, roots, debris) that may interfere with subsequent treatment processes or damage process equipment. Physical separation processes are also used in conjunction with chemical extraction processes to concentrate contaminated media and minimize the volume of media to be treated. Physical separation processes are being evaluated for treatment or.pretreatment of LAOCB soil.

Screening is a mechanical process used to separate particles on the basis of size. Screening utilizes a surface perforated with openings of uniform size upon which soil and/or other media is placed. Particles larger than the screen openings are retained whereas the particles smaller than the screen openings are allowed to pass through.

Screening effectiveness may be affected by the amount of moisture in the feed material. To be effective, the feed material can be either dry or wet, but should not be damp or sticky. Damp or sticky feeds tend to agglomerate and plug screen openings. Screening efficiency is generally increased when using wet feed since the smaller fines may be washed from the larger particles by the passing feed.

A disadvantage of screening dry soil is the potential for airborne particulate emissions (dust); however, airborne emissions can be minimized by completely enclosing the screen and using a dust extraction/treatment system (EPA, 1988d). A disadvantage of screening wet soil is the production of a secondary (liquid) waste stream which would also require subsequent treatment. 
Classification is the separation of particles according to their settling rate in a fluid, usually water. Classifiers typically produce two streams, one containing the less dense, slower settling particles (overflow or slimes) and the other stream that contains the more dense or faster settling particles (underflow or oversize). Hydraulic classifiers use a stream of water or air introduced so that it is flowing in the direction opposite of the settling particles. The more dense particles are capable of settling through the fluid, whereas the less dense particles are entrained in the fluid flow and unable to settle. Mechanical classifiers rely on fluid overflow to entrain slow-settling particles while particles with a higher settling velocity are deposited on the bottom of the equipment and dragged upwards against the flow of liquid by some mechanical means.

Classification technologies have been demonstrated effective and reliable for many years in the uranium mining industry; however, clays and sandy soil with humic materials may be very difficult to process using this technology. In addition, classification processes that use liquid overflows generate an additional (liquid) waste stream that would require subsequent treatment.

Gravity separation devices initiate and maintain particle separation so that the particles are able to move relative to each other within a fluid and thus separate into layers of dense and light materials. Gravity separation devices include jigs, shaking tables, sluices. An innovative gravity separation technology is the heavy media separation process. Heavy media separation process uses heavy liquids (specific gravity $>1$ ) for segregating light and heavy (dense) particles.

Gravity separation processes generally work well with most soil types; however, gravity processes generally use liquid overflows resulting in an additional (liquid) waste stream that would require subsequent treatment.

Although these physical separation technologies have been field demonstrated for extraction of radioactive material from ores, pilot testing would be needed to determine their effectiveness for 
concentrating contaminated media at radiologically contaminated sites. The effectiveness of these technologies for segregating contaminated and uncontaminated soil is questionable since the distribution of radioactive contamination on LAOCB soil is not known. Consequently, it is not known whether LAOCB soil characteristics can be exploited to concentrate radioactive contamination. However, physical separation processes have been demonstrated effective at numerous contaminated sites as a pretreatment method for segregating oversized materials (e.g., rocks, roots, debris) that may interfere with subsequent treatment processes. Of the physical separation techniques reviewed, screening is the most straight forward process requiring readily available equipment and generating minimal secondary wastes when conducted on dry soil. Classification and gravity separation are rejected from further consideration on the basis of effectiveness. Screening can be readily implemented, and associated costs are low. Screening will therefore be retained for consideration as a pretreatment technology for LAOCB soil.

\subsubsection{Disposal of LAOCB Soil and the LAOCB Pipeline}

\section{On-Unit Disposal}

On-unit disposal generally consists of replacing wastes to a disposal unit following ex-situ treatment of the LAOCB soil and in-situ S/S treatment of the LAOCB pipelines. Discussion of ex-situ treatment technologies for LAOCB soil (see Section 2.5.2.5) and in-situ S/S treatment for the LAOCB pipeline (Section 2.4.2.4) included consideration of the disposal of the LAOCB soil and LAOCB pipeline inside the LAOCB following treatment.

\section{Off-Unit Disposal}

Remediation of impacted media and residual materials can potentially be managed at an off-unit location. In accordance with the NCP, corrective measures/remedial alternatives involving offunit disposal must be more cost effective than other remedial actions, and create new waste management capacity, or be necessary to protect public health and welfare or the environment. 


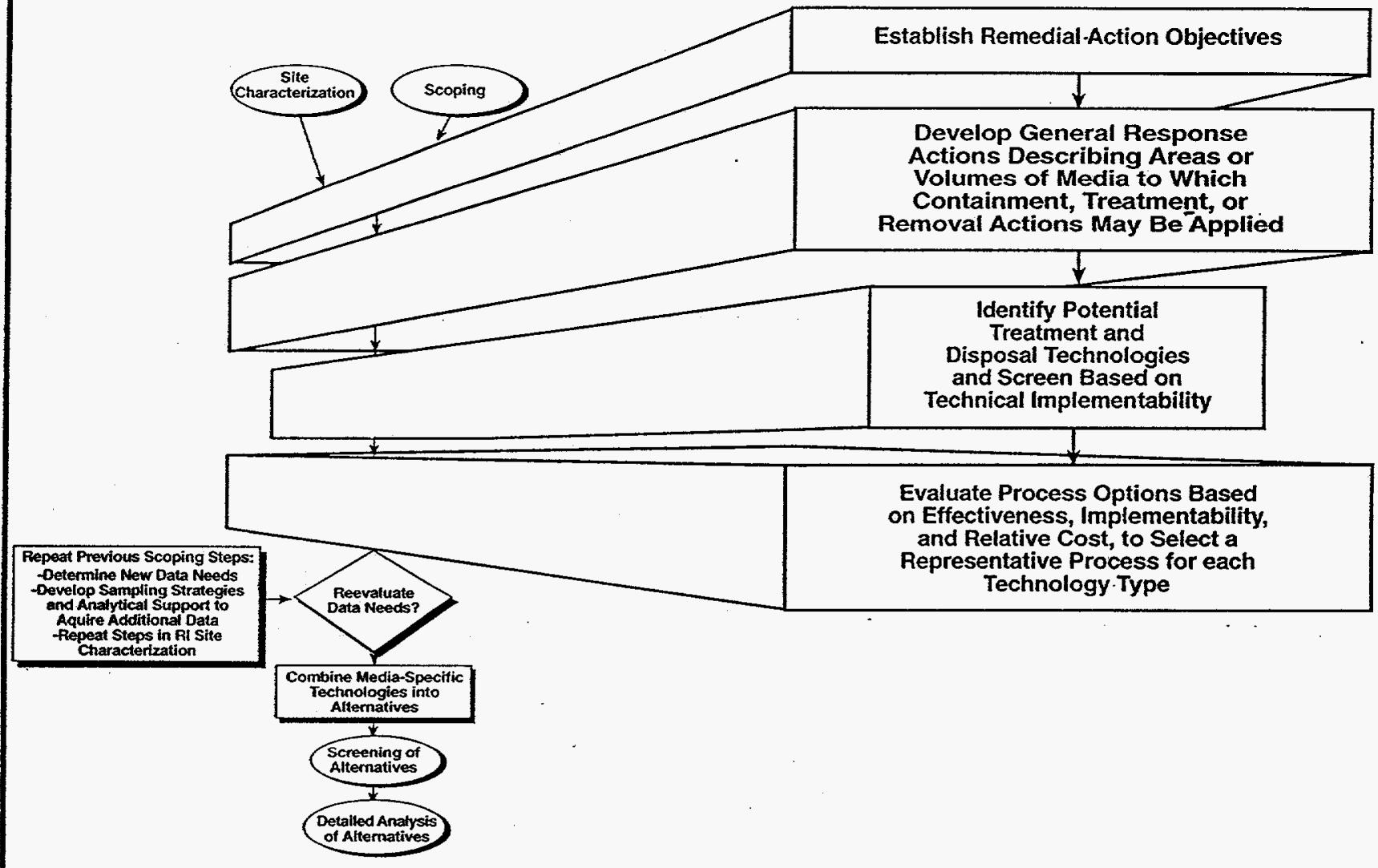

Source: EPA, 1988a

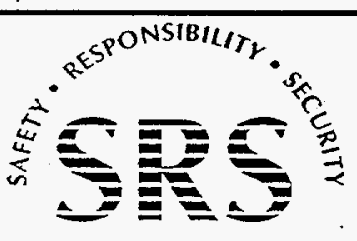

FIGURE 2-1

\begin{tabular}{|c|c|}
\hline & $\begin{array}{c}\text { FIGURE 2-1 } \\
\text { RESPONSE ACTION SELECTION } \\
\text { PROCESS } \\
\text { L-Area Oil and Chemical Basin } \\
\text { Savannah River Site }\end{array}$ \\
\hline
\end{tabular}




\section{Track Mounted Jet-Grout Drilling}

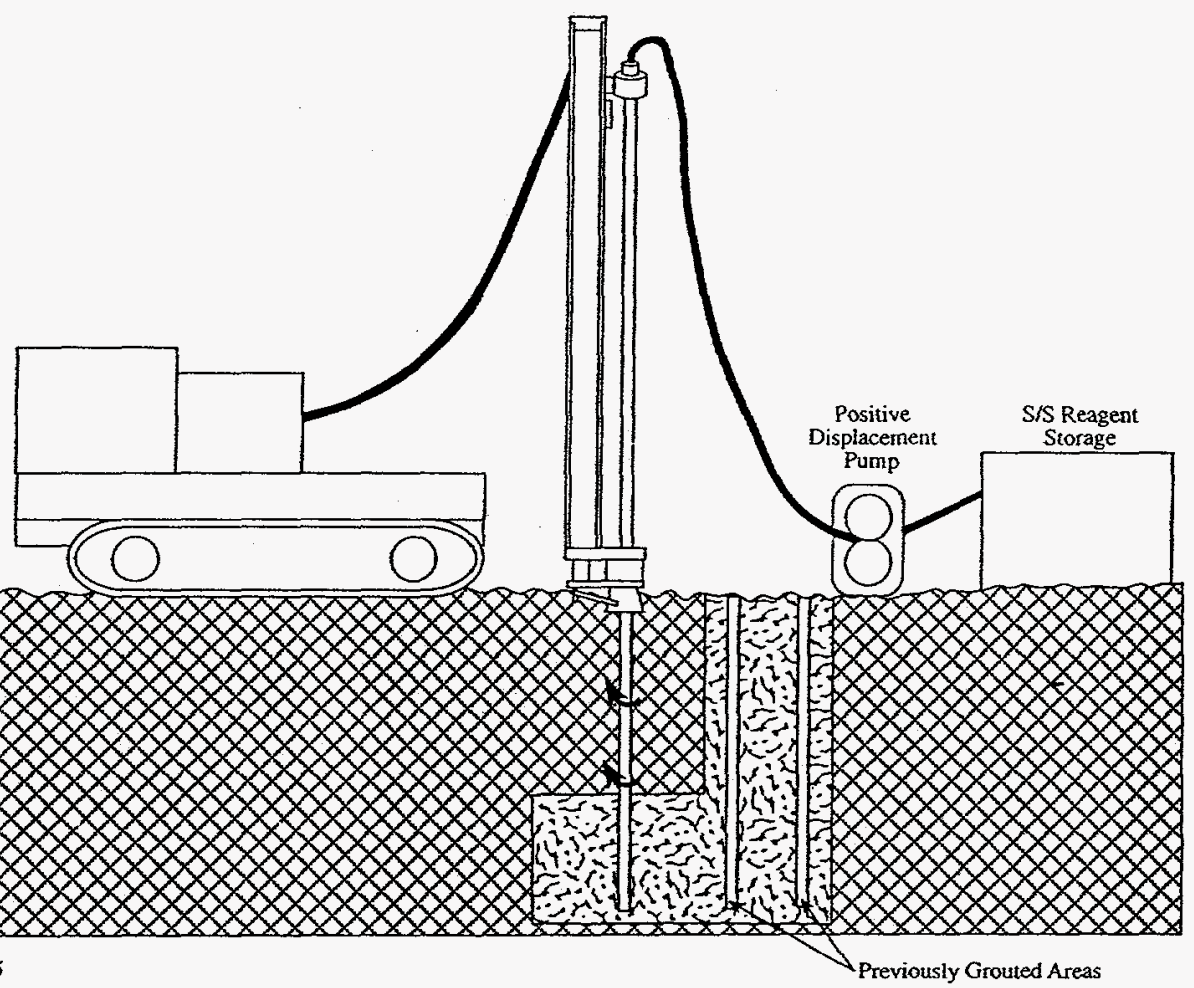

\section{Crane Mounted Soil/Grout Mixing System}

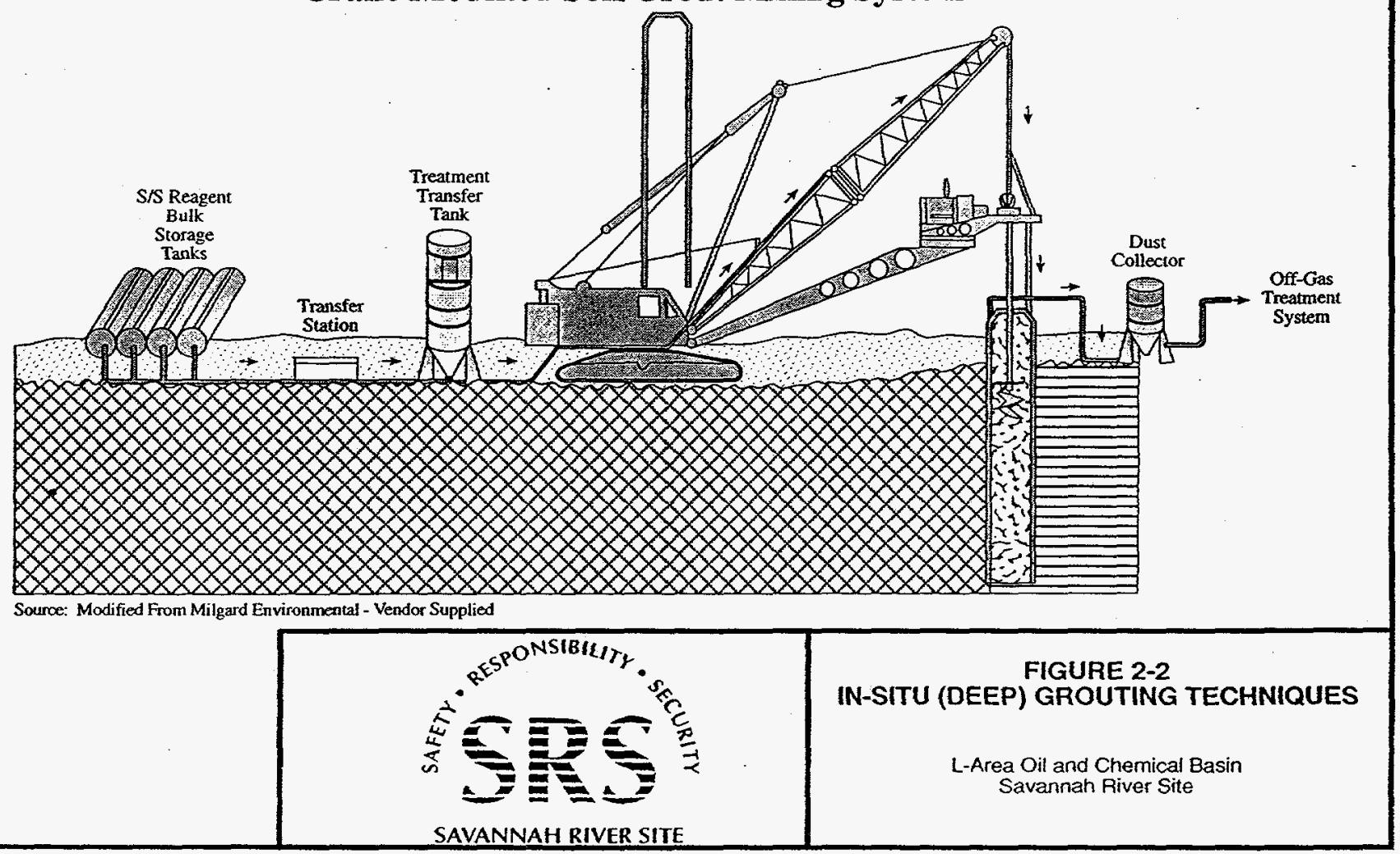


THIS PAGE INTENTIONALLY LEFT BLANK 

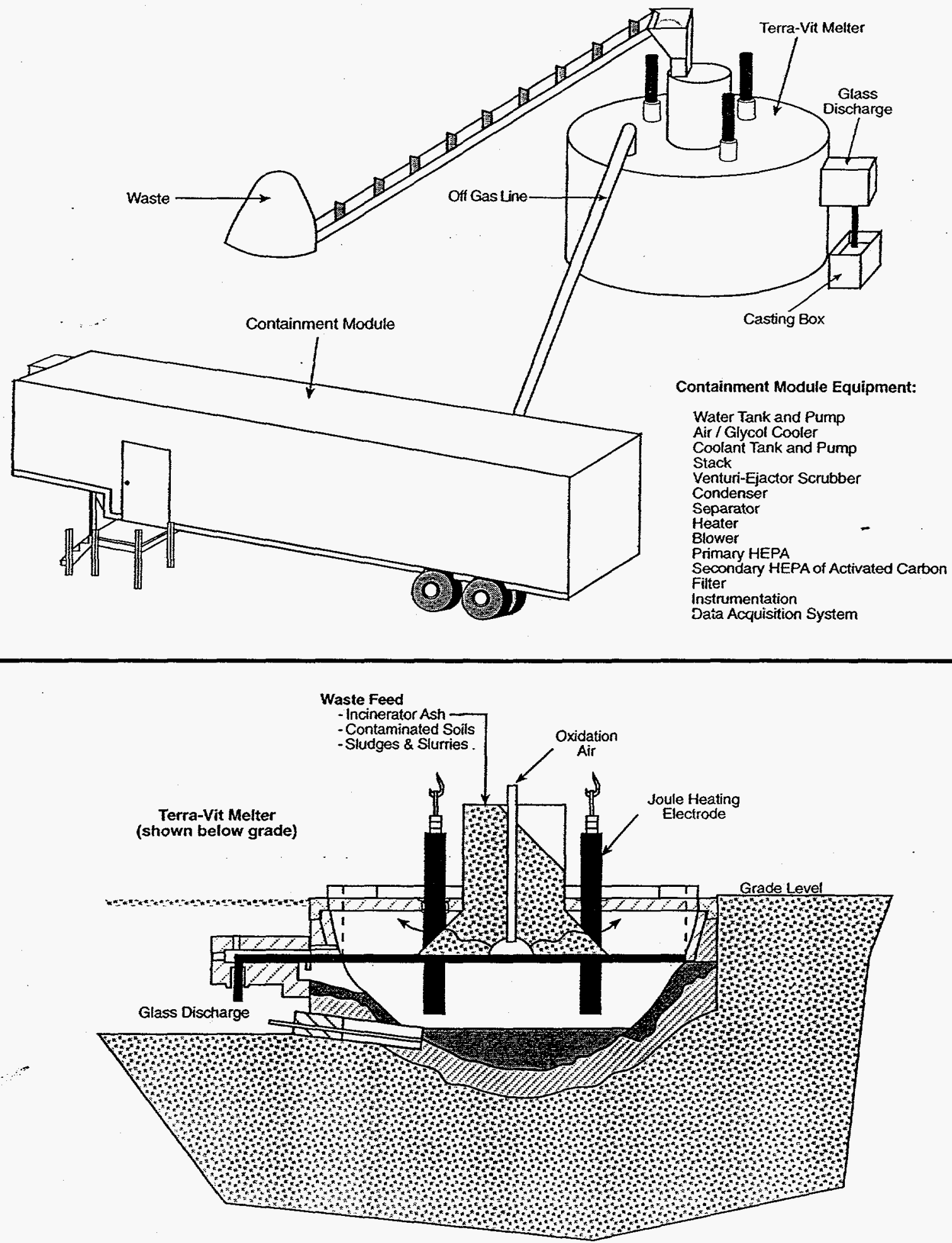

Source: DOE, 1993

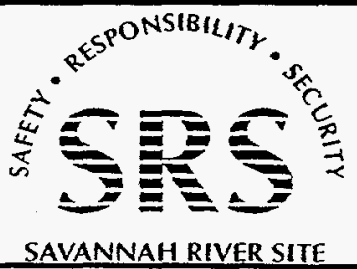

FIGURE 2-3

TERRA-VIT MELTER

EX SITU VITRIFICATION

L-Area Oil and Chemical Basin Savannah River Site 
PHASE I FOCUSED CMS/FS REPORT

L-AREA OIL AND CHEMICAL BASIN
WSRC-RP-96-106, REV. 1.1

FEBRUARY 1997

THIS PAGE INTENTIONALLY LEFT BLANK 


\section{CHAPTER 2}

TABLES 


\section{THIS PAGE INTENTIONALLY LEFT BLANK}


TABLE 2-1

POTENTIAL LOCATION-SPECIFIC ARARs

L-AREA OIL AND CHEMICAL BASIN

SAVANNAH RIVER SITE

SITE FEATURE/LOCATION

Within 61 meters ( 200 feet) of a fault displaced in Holocene time

Within 100-year floodplain

$\hat{\sigma}$

Within floodplain or wetlands

Within area where action may cause irreparable harm, loss

or destruction of significant artifacts

Critical habitat upon which endangered species or threatened species depends

CITATION

REQUIREMENT SYNOPSIS

\section{FEDERAL}

40 CFR 264.18(a)

40 CFR 264.18(b)

Protection of floodplains ( 40 CFR 6, Appendix A); Fish and Wildlife Coordination Act ( 16 USC 661 et seg.); 40 CFR 6.302; Floodplains Executive Order (EO 11988) Wetlands Executive Order

(EO 11990); DOE Compliance

with Flooplain/

Wetlands (10 CFR 1022)

National Historical

Preservation Act (16 USC

Section 469); 36 CFR Part 65

Endangered Species Act of 1973 (16 USC 1531 et seq.); 50 CFR Part 200, 50 CFR Part
New treatment, storage, or disposal of hazardous waste prohibited; applies to RCRA hazardous waste; treatment, storage, or disposal.

Facility must be designed, constructed, operated, and maintained to avoid washout; applies to RCRA hazardous waste; treatment, stored, or disposal.

Action to avoid adverse effects, minimize potential harm, restore and preserve natural and beneficial values; applies to action that will occur in a floodplain, i.e., lowlands, and relatively flat areas adjoining inland and coastal waters and other flood prone areas.

Requires that action be taken to recover and preserve artifacts when alteration of terrain threàtens significant scientific, prehistorical, historical, or archaeologicaldata.

If endangered or threatened species are present, action must be taken to conserve endangered or threatened
CONSIDERATION IN THIS FFS

Not an ARAR since LAOCB is not within 200 feet of a fault displaced in Holocene time.

Not an ARAR since a facility is not being built.

Not an ARAR since LAOCB is not within a floodplain or wetlands area.

Not an ARAR since LAOCB is not a designated archaeological area.

Not an ARAR since endangered or threatened species do not utilize the LAOCB unit. 
TABLE 2-1 (CONTINUED)

POTENTIAL LOCATION-SPECIFIC ARARs

L-AREA OIL AND CHEMICAL BASIN

SAVANNAH RIVER SITE

SITE FEATURE/LOCATION

Habitat of Migratory Birds

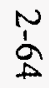

Wetlands

Wildernessarea
CITATION

\section{REQUIREMENT SYNOPSIS}

\section{FEDERAL}

402; Fish and Wildlife

species, including consultation with

Coordination Act (16 USC 661 the Department of Interior.

et seq.); 33 CFR Parts 320-330,

Bald and Golden Eagle

Protection Act (16 USC 668-

668d; 50 CFR 13 and 22)

Migratory Bird Treaty Act (17 If migratory birds are present in the USC 703 et seq.); 50 CFR 10. project area, action must avoid adverse impacts to the species.

Clean Water Act Section 404; For wetlands as defined by U.S. 40 CFR Part 230, 33 CFR Parts Army Corps of Engineers $320-330$

regulations, must take action to prohibit discharge of dredged or fill material into wetlands without permit.

40 CFR Part 6, Appendix A For action involving construction of facilities or management of property in wetlands (as defined by $40 \mathrm{CFR}$ Part 6, Appendix A, section 4(j)), action must be taken to avoid adverse effects, minimize potential harm, and preserve and enhance wetlands, to the extent possible.

Wilderness Act (16 USC 1131 et seg.); 50 CFR 35.1 et seq.
Not an ARAR since migratory birds are not present in the $L A O C B$ area.

Not an ARAR since LAOCB is not within a wetlands area.

Not an ARAR since LAOCB is not within a wetlands area.

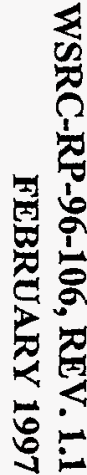




\section{TABLE 2-1 (CONTINUED) \\ POTENTIAL LOCATION-SPECIFIC ARARS \\ L-AREA OIL AND CHEMICAL BASIN \\ SAVANNAH RIVER SITE}

SITE FEATURE/LOCATION

CITATION

\section{REQUIREMENT SYNOPSIS}

CONSIDERATION IN THIS FFS

Within area affecting national wild, scenic, or recreational river

Archaeological Resources

Historical Places

Prehistoric Ruins

Classificationand potential use of an aquifer
Wild and Scenic Rivers Act (16 USC 1271 et seq.); section 7 (a)); 40 CFR 6.302(e)

Archaeological Resources Protection Act of 1979 (16 USC $469 \mathrm{a}$ et seq.); 43 CFR 7 and 36 CFR 296

National Historic Preservation Act of 1966, as amended (16 USC 470 et seg.); 36 CFR 800

If project area contains any site, structure, or object identified in the National Register of Historic Places, action must preserve the historic place.

American Antiquities Act (16 If project area contains prehistoric USC 432 et seg.); 25 CFR 261, ruins, monuments, or objects of 36 CFR 296, and 43 CFR 3-7 antiquity; action must preserve the ruins.

Guidelines for Ground Water Classification,EPA Ground Water Protection Strategy. classificationsin the assessment of remedial response objectives.

section 1271(a), must avoid taking or assisting in action that will have

If archaeological data are present, action must be taken to preserve historic and archaeological data.
Consider Federal and State aquifer

Not an ARAR since LAOCB is not near a scenic river.

(USEPA, 1984; USEPA, 1986)
Not an ARAR since the LAOCB contain no archaeologicalor historic data.

Not an ARAR since the LAOCB area contains no historic sites, structures, or objects.

Not an ARAR since the LAOCB contain no prehistoric ruins.

Potential ARAR for groundwater remediation at the LAOCB. 
TABLE 2-1 (CONTINUED)

POTENTIAL LOCATION-SPECIFIC ARARS

L-AREA OIL AND CHEMICAL BASIN

SAVANNAH RIVER SITE

SITE FEATURE/LOCATION

CITATION

\section{STATE}

Within 100-year floodplain

Wetlands

ڤ́s

疍
Facility located within a 100 -year floodplain must be designed, constructed, and maintained to permit washout of any waste materials.

S.C. Pollution Control Act

Facility must not be located in a

wetland.
Not an ARAR since a facility is not being built.
Not an ARAR since a facility is not being built.

\section{REQUIREMENT SYNOPSIS CONSIDERATION IN THIS FFS}

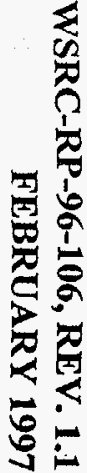


TABLE 2-2

ESTIMATED HUMAN HEALTH RISK-BASED REMEDIAL GOAL OPTIONS

L-AREA OIL AND CHEMICAL BASIN

SAVANNAH RIVER SITE

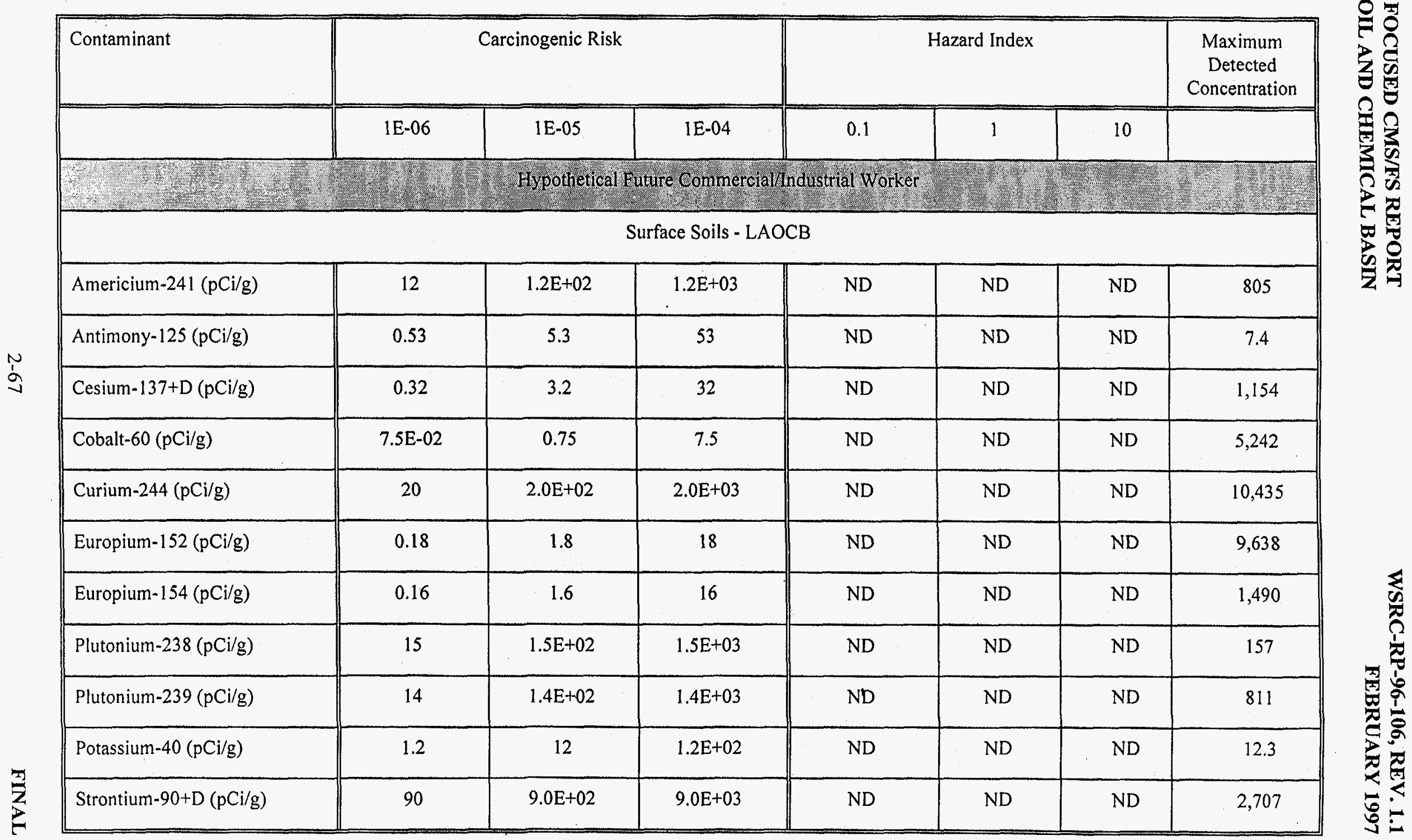


TABLE 2-2 (CONTINUED)

ESTIMATED HUMAN HEALTH RISK-BASED REMEDIAL GOAL OPTIONS

L-AREA OIL AND CHEMICAL BASIN

SAVANNAH RIVER SITE

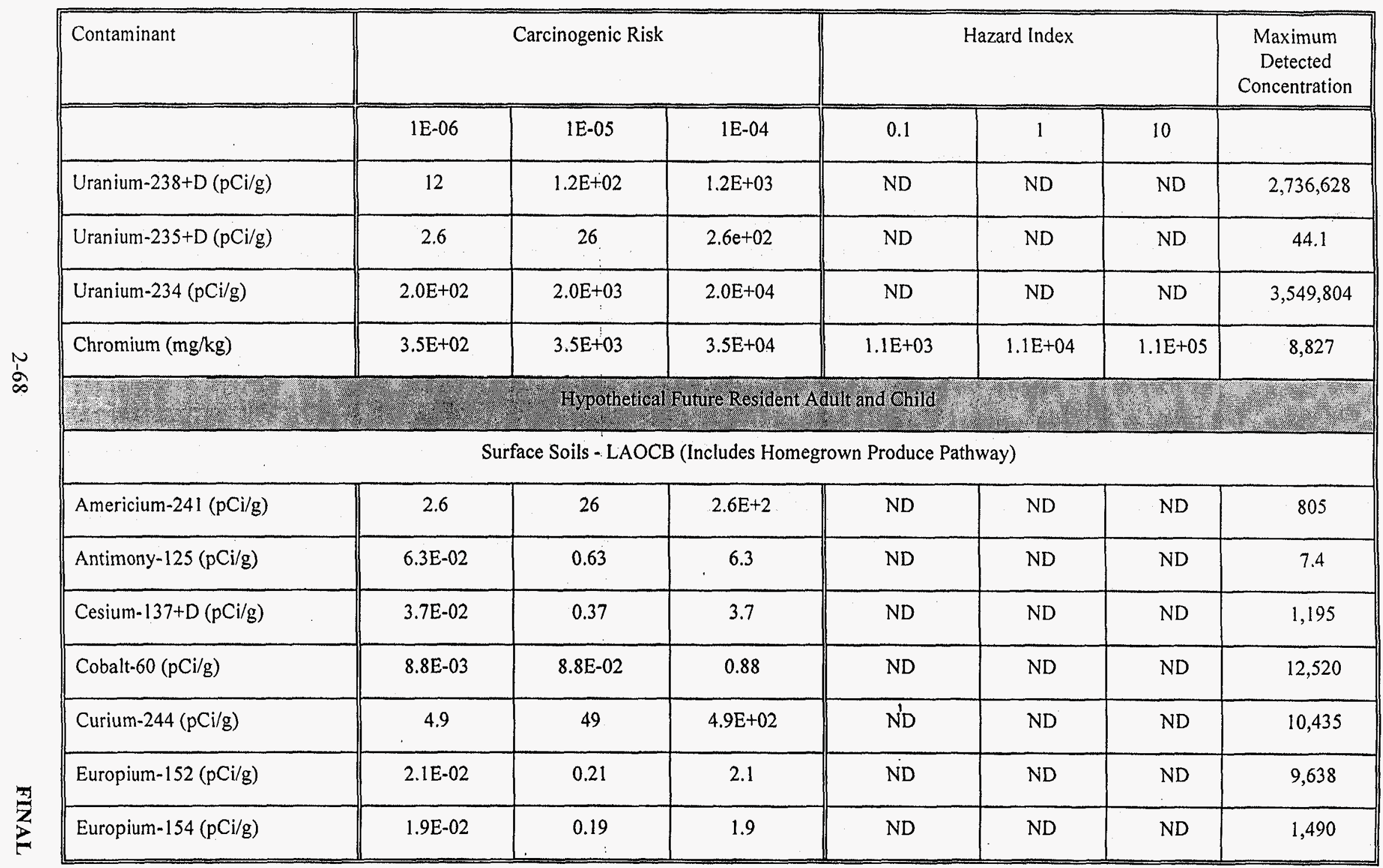


TABLE 2-2(CONTINUED)

ESTIMATED HUMAN HEALTH RISK-BASED REMEDIAL GOAL OPTIONS

L-AREA OIL AND CHEMICAL BASIN

SAVANNAH RIVER SITE

\begin{tabular}{|c|c|c|c|c|c|c|c|}
\hline \multirow[t]{2}{*}{ Contaminant } & \multicolumn{3}{|c|}{ Carcinogenic Risk } & \multicolumn{3}{|c|}{ Hazard Index } & \multirow{2}{*}{$\begin{array}{c}\text { Maximum } \\
\text { Detected } \\
\text { Concentration }\end{array}$} \\
\hline & $1 \mathrm{E}-06$ & $1 \mathrm{E}-05$ & $1 E-04$ & 0.1 & 1 & 10 & \\
\hline Plutonium-238 (pCi/g) & 3.6 & 36 & $3.6 \mathrm{E}+02$ & ND & ND & ND & 157 \\
\hline Plutonium-239 (pCi/g) & 3.4 & 34 & $3.4 \mathrm{E}+02$ & ND & ND & ND & 811 \\
\hline Potassium-40 (pCi/g) & 0.12 & 1.2 & 12 & ND & ND & ND & 12.3 \\
\hline Strontium-90 (pCi/g) & 0.39 & 3.9 & 39 & ND & ND & ND & 2,707 \\
\hline Uranium-238+D (pCi/g) & 1.4 & 14 & $1.4 \mathrm{E}+02$ & ND & ND & ND & $2,736,628$ \\
\hline Uranium-234 (pCi/g) & 25 & $2.5 E+02$ & $2.5 \mathrm{E}+03$ & ND & ND & ND & $3,549,804$ \\
\hline Uranium-235+D (pCi/g) & 0.31 & 3.1 & 31 & ND & ND & ND & 44.1 \\
\hline Aluminum (mg/kg) & ND & ND & ND & $7.8 \mathrm{E}+03$ & $7.8 \mathrm{E}+04$ & $7.8 E+05$ & 57,681 \\
\hline Cadmium (mg/kg) & $1.0 \mathrm{E}+03$ & $1.0 \mathrm{E}+04$ & $1.0 \mathrm{E}+05$ & 1.2 & 12 & $1.2 \mathrm{E}+02$ & 12.3 \\
\hline Chromium (mg/kg) & $1.6 \mathrm{E}+02$ & $1.6 \mathrm{E}+03$ & $1.6 E+04$ & 34 & $3.4 \mathrm{E}+02$ & $3.4 E+03$ & 5,250 \\
\hline Lead $(\mathrm{mg} / \mathrm{kg})$ & ND & ND & ND & 2.8 & 28 & $2.8 \mathrm{E}+02$ & 243 \\
\hline Nickel (mg/kg) & ND & ND & ND & 47 & $4.7 \mathrm{E}+02$ & $4.7 \mathrm{E}+03$ & 395 \\
\hline Vanadium (mg/kg) & ND & ND & ND & 54 & $5.4 \mathrm{E}+01$ & $5.4 \mathrm{E}+02$ & 136 \\
\hline
\end{tabular}


TABLE 2-2 (CONTINUED)

ESTIMATED HUMAN HEALTH RISK-BASED REMEDIAL GOAL OPTIONS

L-AREA OIL AND CHEMICAL BASIN

SAVANNAH RIVER SITE

\begin{tabular}{l|l||c|c|c||c|c|c|c|}
\hline Contaminant & \multicolumn{3}{|c||}{ Carcinogenic Risk } & \multicolumn{3}{|c|}{$\begin{array}{c}\text { Hazard Index } \\
\text { Detected } \\
\text { Concentration }\end{array}$} \\
\hline \hline & $1 \mathrm{E}-06$ & $1 \mathrm{E}-05$ & $1 \mathrm{E}-04$ & 0.1 & 1 & 10 & \\
\hline
\end{tabular}

ND $=$ No Data/Not Applicable

NOTE: Potential carcinogenic risk and noncarcinogenic hazards were within acceptable limits for the current on-unit sampler. No RGOs were estimated for the on-unit sampler exposure pathways. 
TABLE 2-3

LISTING OF POTENTIALLY APPLICABLE REMEDIAL TECHNOLOGIES

L-AREA OIL AND CHEMICAL BASIN SOIL

SAVANNAH RIVER SITE

\begin{tabular}{|c|c|}
\hline General Response Action & Potential Technology \\
\hline No Action & Not Applicable \\
\hline Institutional Controls & Considered in conjunction with other alternatives \\
\hline \multirow[t]{2}{*}{ Containment } & Capping \\
\hline & Vertical Barriers \\
\hline \multirow[t]{3}{*}{ In-Situ Treatment } & Stabilization/Solidification \\
\hline & Vitrification \\
\hline & Electrokinetic Soil Processing \\
\hline \multirow[t]{4}{*}{ Ex-Situ Treatment } & Stabilization/Solidification \\
\hline & Vitrification \\
\hline & Chemical Extraction \\
\hline & Physical Separation \\
\hline \multirow[t]{8}{*}{ Off-Unit Disposal } & SRS Disposal \\
\hline & E-Area Vaults \\
\hline & Mixed Waste Storage Buildings \\
\hline & Soil Consolidation Facility \\
\hline & Off-Site Disposal \\
\hline & Nevada Test Site \\
\hline & Envirocare \\
\hline & Chem-Nuclear Facility \\
\hline
\end{tabular}


TABLE 2-4

LISTING OF POTENTIALLY APPLICABLE REMEDIAL TECHNOLOGIES

L-AREA OIL AND CHEMICAL BASIN PIPELINES

SAVANNAH RIVER SITE

\begin{tabular}{|c|c|}
\hline General Response Action & Potential Technology \\
\hline No Action & Not Applicable \\
\hline Institutional Controls & Considered in conjunction with other alternatives \\
\hline Containment & Capping \\
\hline In-Situ Treatment & Stabilization/Solidification \\
\hline On-Unit Disposal & Disposal inside the LAOCB - combined with in-situ-S/S \\
\hline \multirow[t]{8}{*}{ Off-Unit Disposal } & SRS Disposal \\
\hline & E-Area Vaults \\
\hline & Mixed Waste Storage Buildings \\
\hline & Soil Consolidation Facility \\
\hline & Off-Site Disposal \\
\hline & Nevada Test Site \\
\hline & Envirocare \\
\hline & Chem-Nuclear Facility \\
\hline
\end{tabular}


TABLE 2-5

TECHNOLOGY SCREENING SUMMARY

L-AREA OIL AND CHEMICAL BASIN SOIL

SAVANNAH RIVER SITE

\begin{tabular}{|c|c|c|}
\hline General Response/Technology & Status & Comments \\
\hline No Action & Retained & No action alternative required by the NCP. \\
\hline Institutional Controls & Retained & $\begin{array}{l}\text { Considered in conjunction with other } \\
\text { alternatives. }\end{array}$ \\
\hline Containment/Capping & Retained & Proven effectiveness. \\
\hline \multicolumn{3}{|l|}{ Containment/Vertical Barriers } \\
\hline Slurry Cut-Off Walls & Retained & Effective with hardpan beneath the Basin. \\
\hline $\begin{array}{l}\text { Grout Curtains/Sheet Pile } \\
\text { Walls/Cryogenc Barrers } \\
\end{array}$ & Rejected & Not as effective as slurry cut of walls. \\
\hline In-Situ Treatment/Stabilization-Solidification & Retained & $\begin{array}{l}\text { Proven effectiveness. S/S reagents have been } \\
\text { identified through treatability studies. }\end{array}$ \\
\hline In-Situ Treatment/Vitrification & Retained & Potentially effective on LAOCB media. \\
\hline In-Situ TreatmentElectrokinetics & Rejected & $\begin{array}{l}\text { Implementability and effectiveness-not } \\
\text { demonstrated full-scale. }\end{array}$ \\
\hline Ex-Situ Treatment/Stabilization-Solidification & Retained & $\begin{array}{l}\text { Demonstrated effective. } \mathrm{S} / \mathrm{S} \text { reagents have been } \\
\text { identified through treatability studies. }\end{array}$ \\
\hline Ex-Situ Treatment/Vitrification & Retained & Demonstrated effective. \\
\hline Ex-Situ Treatment Chemical Extraction & Rejected & Effectiveness: Does not meet BRC citeriat \\
\hline Ex-Situ Treatment/Physical Separation & Retained & $\begin{array}{l}\text { Screening effective as pretreatment of soil as } \\
\text { needed. }\end{array}$ \\
\hline \multicolumn{3}{|l|}{ Off-Unit Disposal } \\
\hline At SRS: E-Area Vaults & Rejected & Insufficient capacity \\
\hline $\begin{array}{l}\text { At SRS. Mixed Waste Storage } \\
\text { BuildingS }\end{array}$ & Rejected & $\begin{array}{l}\text { Waste acceptance criteria will not allow disposal } \\
\text { of soil. Loing term effectiveness limited. }\end{array}$ \\
\hline $\begin{array}{l}\text { At SRS: Soil Consolidation } \\
\text { Facility }\end{array}$ & Retained & Innovative technology. \\
\hline $\begin{array}{ll}\text { Off-Site: Nevada Test Site } \\
\text { (Nevada) }\end{array}$ & Retained & $\begin{array}{l}\text { Available capacity and should accept LAOCB } \\
\text { wastes. }\end{array}$ \\
\hline $\begin{array}{l}\text { Off-Site Chem-Nuclear (Banwill, } \\
\text { SC) }\end{array}$ & Rejected & Cost prohibitive, \\
\hline Off-Site: Envirocare (Utah) & Retained & $\begin{array}{l}\text { Available capacity and should accept LAOCB } \\
\text { wastes. }\end{array}$ \\
\hline
\end{tabular}


TABLE 2-6

SUMMARY OF RETAINED TECHNOLOGIES

L-AREA OIL AND CHEMICAL BASIN SOIL SAVANNAH RIVER SITE

\begin{tabular}{|l|}
\hline \multicolumn{1}{|c|}{ General Response/Technology } \\
\hline No Action \\
Institutional Controls \\
\hline Containment/Capping \\
\hline Containment/Slurry Cut-Off Wall \\
\hline In-Situ Treatment/Stabilization/Solidification \\
\hline In-Situ Treatment/Vitrification \\
\hline Ex-Situ Treatment/Stabilization/Solidification \\
\hline Ex-Situ Treatment/Screening \\
\hline Ex-Situ Treatment/Vitrification \\
\hline Off-Unit Disposal \\
\hline Soil Consolidation Facility \\
\hline Nevada Test Site \\
\hline Envare (Utah) \\
\hline
\end{tabular}


TABLE 2-7

TECHNOLOGY SCREENING SUMMARY

L-AREA OIL AND CHEMICAL BASIN PIPELINE SAVANNAH RIVER SITE

\begin{tabular}{|c|c|c|}
\hline General Response/Technology & Status & Comments \\
\hline No Action & Retained & No action alternative required by the NCP. \\
\hline Institutional Controls & Retained & $\begin{array}{l}\text { Considered as either a stand-alone alternative but } \\
\text { may be used in conjunction with other } \\
\text { alternatives. }\end{array}$ \\
\hline Containment/Capping & Retained & Proven effective. \\
\hline In-Situ Treatment/Stabilization-Solidification & Retained & Proven effective. \\
\hline \multicolumn{3}{|l|}{ On-Unit Disposal } \\
\hline Inside the LAOCB & Retained & $\begin{array}{l}\text { Effective - may be combined with other } \\
\text { alternatives to increase effectiveness. }\end{array}$ \\
\hline \multicolumn{3}{|l|}{ Off-Unit Disposal } \\
\hline At SRS E-Area Vaults & Rejected & Insufficient capacity. \\
\hline $\begin{array}{l}\text { At SRS: Mixed Waste Storage } \\
\text { Buildings }\end{array}$ & Rejected & $\begin{array}{l}\text { Waste acceptance criteria will not allow disposal } \\
\text { of soil. Long tem effectiveness limited. }\end{array}$ \\
\hline $\begin{array}{l}\text { At SRS: Soil Consolidation } \\
\text { Facility }\end{array}$ & Retained & Innovative technology. \\
\hline $\begin{array}{l}\text { Off-Site: Nevada Test Site } \\
\text { (Nevada) }\end{array}$ & Retained & Not as effective as Envirocare facility. \\
\hline $\begin{array}{l}\text { Off-Site: Chem-Nuclear (Barnwell, } \\
\mathrm{SC} \text { ) }\end{array}$ & Rejected & Cost prohibitive: \\
\hline Off-Site: Envirocare (Utah) & Retained & $\begin{array}{l}\text { Available capacity and should accept LAOCB } \\
\text { wastes. }\end{array}$ \\
\hline
\end{tabular}


TABLE 2-8

SUMMARY OF RETAINED TECHNOLOGIES

L-AREA OIL AND CHEMICAL BASIN PIPELINE SAVANNAH RIVER SITE

\begin{tabular}{|l||}
\hline General Response/Technology \\
No Action \\
Institutional Controls \\
Containment/Capping \\
In-Situ Treatment/Stabilization/Solidification \\
\hline On-Unit Disposal \\
\hline Inside the LAOCB \\
\hline Off-Unit Disposal \\
\hline Soil Consolidation Facility \\
Nevada Test Site \\
Envirocare (Utah) \\
\hline
\end{tabular}


In Section 2.0, remedial technologies were screened and evaluated on an individual basis without regard to their part in a comprehensive remedial action. In Section 3.1, the retained technologies are assembled into functional alternatives which address LAOCB soil and subsequently evaluated. In Section 3.2, the technologies retained for remediation of the LAOCB pipelines are assembled into functional alternatives and evaluated. Those alternatives that are retained after the screening are subjected to detailed analysis in Section 4.0.

\subsection{Development and Evaluation of Remedial Alternatives for LAOCB Soil}

Potential remedial alternatives have been developed to address LAOCB waste unit contaminated soil. The soil adjacent to the Basin and soil associated with the pipeline do not require remediation. Vegetation in the vicinity of the Basin has already been managed. The NCP requires that a range of alternatives that include treatment to reduce toxicity, mobility, or volume of contaminants be developed and evaluated. The range of alternatives developed includes alternatives which immobilize residual chemicals or reduce the volume of contaminants or contaminated media and, to the maximum extent feasible, eliminate or minimize the need for long-term management. Alternatives have also been developed which involve little or no treatment, but which provide protection to human health and the environment by preventing or controlling exposure to the contaminants through engineering or institutional controls. As required by the NCP, the No Action alternative is provided as a baseline for comparison.

In Section 3.1.2, remedial alternatives formulated in Section 3.1.1 undergo a screening evaluation to reduce the number of alternatives that subsequently will undergo a more thorough and extensive detailed analysis. Alternatives retained after screening in this section are subjected to a detailed evaluation in Section 4.0. 


\subsubsection{Development of Remedial Alternatives}

The following remedial alternatives address contamination in LAOCB soil. The potential remedial alternatives that address $L A O C B$ soil contamination and the rationale for their development are:

Alternative S-1: $\quad$ No Action

Rationale: The No Action alternative is required by the NCP.

Alternative S-2: $\quad$ Backfill and Cap LAOCB

Rationale: Alternative S-2 offers capping, which satisfies the general response of containment. While Alternative S-2 minimizes waste handling, it offers no reduction in waste toxicity, mobility, or volume of the waste.

Alternative S-3: Install Slurry Cut-Off Wall, Backfill and Cap LAOCB

Rationale:

Alternative S-3 also addresses the general response of containment, but includes the installation of vertical walls to further contain soil-borne contaminants. Alternative S-3 minimizes waste handling and decreases contaminant migration, but offers no reduction in waste toxicity, mobility; or the volume of the waste.

Alternative S-4: In-Situ Stabilize, Backfill and Cap the LAOCB

Rationale:

Alternative S-4 addresses the general response of in-situ treatment. This alternative focuses on in-situ treating/immobilizing the most accessible and contaminated soil so as to minimize waste handling. 
Alternative S-5: In-Situ Vitrify, Backfill and Cap the LAOCB

Rationale:

Alternative S-5 involves an innovative technology that addresses the general response of in-situ treatment. Alternative S-5 focuses on treating/immobilizing all known contaminated soil. Vitrification offers the potential for superior long-term effectiveness over grouting. The tradeoff is greater cost.

Alternative S-6: Excavate LAOCB Soil, Stabilize Soil Ex-Situ, Place Treated Soil into LAOCB, Backfill and Cap LAOCB

Rationale:

Alternative S-6 addresses the general responses of ex-situ treatment and containment. This alternative focuses on treating/immobilizing the most accessible and contaminated soil. This alternative requires more waste handling, but offers confirmation that treatment of contaminated media achieves a minimum treatment standard.

Alternative S-7: Excavate LAOCB Soil, Ex-Situ Vitrify Soil, Place Treated Soil into LAOCB, Backfill and Cap LAOCB

Rationale:

Alternative S-7 involves an innovative technology that addresses the general response of ex-situ treatment. Alternative S-7 focuses on treating/immobilizing the most accessible and contaminated soil. In addition to confirmation that all soil exceeding minimum treatment standards have been treated, vitrification offers the potential for superior long-term effectiveness over grouting. However, ex-situ vitrification will require more waste handling than in-situ treatment and has a significantly greater cost relative to grouting. 
Alternative S-8: Excavate LAOCB Soil, Dispose of the Soil at a SRS Soil Consolidation Facility

Rationale: Alternative S-8 involves the disposal of contaminated soil in a conceptual Soil Consolidation Facility and addresses the general response of off-unit disposal. While this alternative offers complete containment of the waste, off-unit disposal does not offer waste treatment that would reduce the toxicity, mobility, or volume of the waste and would involve waste handling.

Alternative S-9: $\quad$ Excavate LAOCB Soil, Dispose of the Soil at the NTS Facility

Rationale:

Alternative S-9 involves the landfilling of contaminated soil and addresses the general response of off-unit disposal. While this alternative offers containment of the waste, it does not offer waste treatment that would reduce the toxicity, mobility, or volume of the waste. This alternative would involve significant waste handling.

\begin{abstract}
Alternative S-10: $\quad$ Excavate LAOCB Soil, Dispose of the Soil at the Envirocare Facility
Rationale:

Alternative S-10 involves the landfilling of contaminated soil and addresses the general response of off-unit disposal. While this alternative. offers containment of the waste, it does not offer waste treatment that would reduce the toxicity, mobility or volume of the waste, or otherwise decrease the inherent threats or risks associated with the waste. This alternative would also involve significant waste handling.
\end{abstract}

\title{
3.1.2 Screening of Remedial Alternatives
}

In accordance with the NCP, it is desirable, when practicable, to offer a range of diverse remedial alternatives to select from during detailed analysis. In order to provide a range of potential 
alternatives for detailed analysis, at least one alternative will be retained that represents each of the following general response actions:

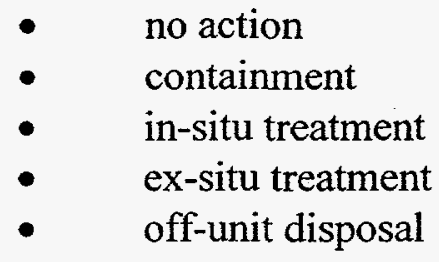

The general response action of institutional control is not included in this list since it was evaluated as not being an effective stand-alone remedy during the technology screening; however, institutional controls are inexpensive and provide an effective means of deterring human access to LAOCB related contamination. It is for these reasons that institutional controls are a common component of all alternatives except the No Action alternative and the alternatives which result in unrestricted use of the unit.

Some alternatives are comprised of two or more general response actions (e.g., in-situ treatment and containment). In such cases, the alternative will be classified as a function of the primary general response action or component of the alternative that enhances its effectiveness over similar alternatives. For example, an alternative that involves in-situ grouting of soil and capping will fall under the general response of in-situ treatment, since in-situ grouting will enhance the alternative's effectiveness over an alternative that involves capping only. The capping-only alternative and the slurry cut-off wall and capping alternative would fall under the general response of containment.

At the alternatives screening point in the CMS/FS process, alternatives will be evaluated more generally than during the detailed analysis; however, screening evaluations will be sufficiently detailed to distinguish among similar types of alternatives. Comparisons will be made among alternatives having a common general response action. To ensure that a range of approaches are considered, at least one (i.e., the most promising) alternative from each general response action 
will be retained for detailed analysis. During comparative analysis (Section 4.3), comparisons among alternatives will differentiate across the entire range of alternatives.

EPA CERCLA guidance for the FS process provides three broad criteria against which whole alternatives are evaluated during the screening process (EPA, 1988a). The criteria are:

- $\quad$ effectiveness

- implementability

- cost

For an alternative to be effective, it must meet at least one remedial action objective based on the protection of human health and the environment. Alternatives which do not provide adequate protection of the environment, public health, and public welfare (i.e., meet remedial action objectives), or do so to a much lesser extent than a comparable alternative, will be rejected and will not be considered for detailed analysis.

Implementability addresses both the technical and institutional feasibility of constructing, operating, and maintaining components of an alternative. Under this criterion, alternatives are evaluated based on the technical feasibility to construct, reliably operate, and meet technologyspecific regulations for process options until a remedial action is completed. Alternatives that involve innovative technologies are retained as long as there is "reasonable belief" that the alternative will offer better treatment performance, fewer adverse impacts, or lower costs for similar levels of performance, over alternatives that involve conventional technologies. Alternatives that are clearly less technically or administratively feasible than comparable alternatives may be removed from further consideration.

A quantitative cost evaluation is provided so that cost comparisons can be made among similar types of alternatives. The costs for alternatives are developed for comparison purposes only and are not intended to forecast a budgetary estimate of actual expenditures. Those alternatives that 
demonstrate comparable levels of effectiveness and implementability as other alternatives but at significantly greater cost will be eliminated. Similarly, alternatives that are equivalent in cost but are clearly less effective than other comparable alternatives will also be rejected. Otherwise, cost will not be used as a criterion to screen alternatives at this point in the CMS/FS process.

EPA RCRA guidance for the CMS process indicates that the screening process should eliminate corrective measures that may prove infeasible to implement, that rely on technologies unlikely to perform satisfactorily or reliably, or that do not achieve the remedial action objectives within a reasonable time period (EPA, 1988c). These screening requirements are discussed in detail in Section 2.6.1 of this document.

The following subsections present a description of potential alternatives and evaluate each alternative based on the screening criteria discussed above. Alternatives retained after this screening will undergo detailed analysis of alternatives in Section 4.0.

\subsubsection{Alternatives Under the General Response of No Action}

Listed below is a description, screening, and evaluation of Alternative S-1. Alternative S-1 is the only alternative that is categorized under the general response of No Action.

\section{Alternative S-1: No Action}

Alternative S-1 is a true No Action alternative. Under this alternative, no remedial efforts would be conducted to remove, treat, or otherwise lessen the toxicity, mobility, or affected volume of contaminated media. Only those institutional controls that currently exist at SRS would be implemented under the No Action alternative. 
Access to SRS is controlled at primary roads by continuously manned barricades. Other roads entering the site are closed to traffic by gates or barriers. The entire SRS facility is surrounded by an exclusion fence, except along the Savannah River. The site is posted against trespassing under Federal and State statutes. The LAOCB is surrounded by a six foot high exclusion fence which is posted as a control area (CA) and, therefore, access directly to the Basin is further controlled beyond that of site security. Consequently, the No Action alternative would satisfy short-term protectiveness of human health through the existing institutional controls at SRS.

Under the No Action alternative, radioactive decay would be the primary mechanism providing a reduction in ${ }^{137} \mathrm{Cs}$ and ${ }^{60} \mathrm{Co}$ concentration within the soil. The maximum concentration of ${ }^{137} \mathrm{Cs}$ within the LAOCB sludge is $1,154 \mathrm{pCi} / \mathrm{g}$ (Table 2-2). The effective external dose equivalent based on a one year exposure would be $2,707 \mathrm{mrem} / \mathrm{year}$ (Appendix A). This is a very conservative estimate since the dose is calculated for a person exposed to Basin soil for 365 days and 24 hours/day. For protectiveness of human health, 40 CFR 192 and DOE Order 5400.5 specify an allowable dose equivalent to the public of $25 \mathrm{mrem} / \mathrm{year}$ and $100 \mathrm{mrem} / \mathrm{year}$, respectively. Based on the half-life of ${ }^{137} \mathrm{Cs}$ ( 30 years; Table 1-3), the estimated time for radioactive decay to reduce the concentration of ${ }^{137} \mathrm{Cs}$ so as not to cause an effective dose equivalent greater than $25 \mathrm{mrem} /$ year or $100 \mathrm{mrem} /$ year is approximately 166 years and 106 years, respectively (Figure 3-1a). The maximum concentration of ${ }^{60} \mathrm{Co}$ within the LAOCB sludge is $5,242 \mathrm{pCi} / \mathrm{g}$ (Table 2-2). The effective external dose equivalent based on a one year exposure would be $79,977 \mathrm{mrem} / \mathrm{year}$ (Appendix A). Based on the half-life of ${ }^{60} \mathrm{Co}$ (5.26 years; Table 1-3), the estimated time for radioactive decay to reduce the concentration of ${ }^{60} \mathrm{Co}$ so as not to cause an effective dose equivalent greater than $25 \mathrm{mrem} / \mathrm{year}$ or $100 \mathrm{mrem} / \mathrm{year}$ is approximately 41 years and 30 years, respectively (Figure 3-1a). Following the required 106 to 166 years required for ${ }^{137} \mathrm{Cs}$ to decay and not cause an effective dose above the regulatory limits, residual radioactivity from ${ }^{60} \mathrm{Co}$ would be negligible. 
Long-term protectiveness of human health would be achieved under the No Action alternative as long as existing institutional controls are maintained for at least 166 years. Although institutional controls are likely to be retained at SRS for the foreseeable future, the controls cannot be guaranteed for the length of time necessary for radioactive constituents to decay to levels protective of human health. Therefore, whether the No Action alternative can achieve long term protectiveness of human health is questionable. Alternative S-1 does not meet any remedial action objectives.

The clay layer (hardpan) underlying the Basin provides an adequate barrier that prevents the migration of primary and secondary COCs through vadose zone soil. No additional efforts would be taken under this alternative to contain LAOCB contamination or contaminated media. The No Action alternative does not provide a mechanism for verifying whether contaminants leach to groundwater in the future.

The No Action alternative would require no construction, specialized equipment, or technical specialists and could therefore be implemented immediately. If implemented, No Action would likely prompt public concern for protection of human health and the environment.

The only costs associated with the No Action alternative would be a review of remedy every five years for 30 years as required by the NCP. Five year remedy reviews are required for remedial actions that result in hazardous substances, pollutants, or contaminants remaining at the waste unit above levels that allow for unlimited use and unrestricted exposure. No long-term operation and maintenance costs would be associated with the No Action alternative. Estimated costs associated with the No Action alternative are summarized below.

$\begin{array}{llr}\text { Capital Costs } & \$ & 0 \\ \text { O\&M Costs } & \$ 280,000 \\ \text { Total Costs } & \$ 280,000\end{array}$


Costs for this alternative are provided in Appendix B.

\section{Evaluation of Alternative S-1}

The No Action alternative would be protective of human health as long as existing institutional controls are maintained at SRS and would be protective of the environment since the clay layer (hardpan) provides a barrier to migration. However, neither the institutional controls nor the minimization of the leachability of the contaminants could be guaranteed in the future. Therefore, Alternative S-1 does not meet any remedial action objectives. However, based on the NCP requirements a No Action alternative must be retained as a baseline alternative against which all other alternatives can be compared. Alternative S-1 will be retained for detailed analysis.

\subsubsection{Alternatives Under the General Response of Containment}

Following is a description, screening, and evaluation of Alternatives S-2 and S-3.

\section{Alternative S-2: Backfill and Cap LAOCB}

Alternative S-2 involves backfilling and constructing a cap over the LAOCB. The LAOCB would first be backfilled to grade using common fill dirt from a source outside of the LAOCB waste unit. If present, standing water would be absorbed by the backfill media. The approximate volume of backfill required is estimated to be $5,300 \mathrm{~m}^{3}(170 \mathrm{ft} \times 96 \mathrm{ft} \times 11.5 \mathrm{ft}=$ $190,000 \mathrm{ft}^{3}$ ). After sufficient compaction, an engineered cap would then be constructed over the LAOCB, covering an area of approximately $2,000 \mathrm{~m}^{2}\left(2.2 \mathrm{E} 4 \mathrm{ft}^{2}\right)$. The cap would have dimensions of $190 \mathrm{ft} \times 116 \mathrm{ft}$ which extends four feet beyond each side of the LAOCB berm (Figures 3-2a and 3-2b). Cap design would be evaluated and selected during Corrective Measures/Remedial Design. Selection of a cap design would be based upon protecting human 
health and the environment. Secondary considerations in the selection of a cap design would include cost and long-term maintenance requirements. Institutional controls such as fences, warning signs, security, and/or property deed restrictions would be implemented and maintained as a component of Alternative S-2.

A properly engineered cap would function as a physical barrier to prevent direct human exposure to soil-borne contamination and thus be protective of human health and the environment. Capping is a performance based engineering approach since it does not reduce the total mass of COCs and can not achieve RGOs. However, as shown in Figure 3-1b, only $0.9 \mathrm{~m}(3 \mathrm{ft})$ of soil cover is required to reduce the annual effective dose from continuous exposure to the ${ }^{137} \mathrm{Cs}$ and ${ }^{60} \mathrm{Co}$ in the Basin by over 99 percent and within regulatory and DOE limits. In addition, the cap would minimize infiltration and subsequent leaching of contamination from unsaturated soil to the groundwater. Therefore, Alternative S-2 meets the remedial action objectives of reducing surficial risks to human health and the environment, and reducing the leaching of tritium and primary and secondary COCs to unit groundwater. In general, cap construction is a straightforward process requiring minimal, if any, disturbance of contaminated LAOCB soil. Consequently, short-term risks to the health of remedial workers would be minimal. If properly constructed and maintained, caps can provide effective long-term protection to human health and the environment.

Alternative S-2 would not involve any form of treatment that would reduce the toxicity, mobility, or volume of contaminants or contaminated media. However, capping would effectively reduce contaminant mobility by minimizing infiltration and contaminant leaching, thereby reducing inherent risks associated with the contamination. Additionally, the clay layer (hardpan) beneath the Basin has proven to be an adequate barrier to $\mathrm{COC}$ migration.

In general, caps are readily implementable. Depending upon the cap design selected, -Alternative S-2 would require readily available materials (e.g., topsoil, clay, gravel, geosynthetics), and the 
use of conventional earthmoving equipment (e.g., backhoes, dump trucks, bull dozers, compactors). Numerous qualified contractors who can competitively bid on the design and construction of an engineered cap are available. Caps have been commonly used at other lowlevel radioactive and mixed waste sites and generally do not elicit public concerns.

Costs associated with Alternative S-2 include the labor and materials needed to construct the cap as well as operation and maintenance for 30 years. These costs include a review of remedy every five years for 30 years as required by the NCP. Five year remedy reviews are required for remedial actions that result in hazardous substances, pollutants, or contaminants remaining at the waste unit above levels that allow for unlimited use and unrestricted exposure. Estimated costs associated with Alternative S-2 are summarized below.

$\begin{array}{ll}\text { Capital Costs } & \$ 1,000,000 \\ \text { O\&M Costs } & \$ 4430,000 \\ \text { Total Costs } & \$ 1,430,000\end{array}$

Costs for this alternative are provided in Appendix B.

Alternative S-3: Install Slurry Cut-Off Walls around the LAOCB; Backfill and Cap LAOCB

Alternative S-3 is similar to Alternative S-2 in that it involves the general response of containment. However, in addition to backfilling and capping the LAOCB, vertical cut-off walls would be installed at the perimeter of the cap area. For slurry walls, soil would be excavated using conventional earth moving equipment to form a trench approximately $0.91 \mathrm{~m}$ by $5.5 \mathrm{~m}$ deep ( $3 \mathrm{ft}$ by $18 \mathrm{ft}$ ) surrounding the LAOCB. As soil is excavated, a soil-bentonite or cementbentonite slurry will be added to support the walls of the excavation. Upon setting, the slurry will form a low-permeability wall. At 4.9 to $5.5 \mathrm{~m}$ (16 to $18 \mathrm{ft}$ ) depth the "AA" Horizon should be encountered. By installing the slurry walls to this depth, the walls will meet the naturallyoccurring clayey zone and will form a low-permeability complete containment unit. Following 
installation of the walls, the LAOCB would be backfilled to grade using common fill dirt from an outside source.

After backfilling and compaction, an engineered cap would then be constructed over the LAOCB. Cap design would be evaluated and selected during detailed design. Selection of a cap design would be based upon protecting human health and the environment. Secondary considerations in the selection of a cap design would include cost and long-term maintenance requirements. Institutional controls such as fences, warning signs, security monitors, and/or property deed restrictions would be implemented and maintained as a component of Alternative S-3.

Alternative S-3 would be protective of human health and the environment. As discussed under Alternative S-2 the cap would function as a physical barrier to prevent direct human exposure to contamination, and a reduced risk associated with external exposure through shielding. Both the slurry walls and cap would minimize infiltration of precipitation into the Basin and reduce leaching of primary and secondary COCs into unit groundwater. In addition, the slurry walls, coupled with the identified "AA" horizon clay underlying the basin floor, would be especially effective at preventing leaching of contaminants to the groundwater by simple containment. Therefore, Alternative S-3 meets the remedial action objective of reducing risks to human health and mitigating the release of tritium and primary and secondary COCs to groundwater at the unit. Short-term risk associated with Alternatives S-2 and S-3 should be equivalent if backfill material is added to the Basin prior to initiating slurry wall or cap construction. Slurry wall and cap construction under Alternative S-3 is a straight-forward process requiring minimal disturbance of LAOCB soil. If properly constructed and maintained, slurry walls and caps can provide effective long-term protection of human health and the environment.

Alternative S-3 would not involve any form of treatment that would reduce the toxicity, mobility, or volume of contaminants or contaminated media. However, cut-off walls and capping would 
effectively reduce contaminant mobility by minimizing infiltration and contaminant leaching, thereby reducing inherent risks posed by the soil-borne contamination.

Installation of a slurry cut-off wall and construction of a cap over the LAOCB soil is readily implementable. Depending upon the slurry wall components and the selected cap design, Alternative S-3 would require readily available materials (e.g., bentonite, topsoil, clay, gravel, geosynthetics), the use of conventional earthmoving equipment (e.g., trackhoes, backhoes, dump trucks, bull dozers, compactors), and appropriate health and safety clothing and monitoring equipment. Numerous qualified contractors who can competitively bid on the construction of slurry walls and an engineered cap are available.

Costs associated with Alternative S-3 include the labor and materials needed to construct the slurry walls and cap. Also included in the costs is the operation and maintenance of the cap for 30 years. Included in these costs is a review of remedy every five years for 30 years as required by the NCP. Five year remedy reviews are required for remedial actions that result in hazardous substances, pollutants, or contaminants remaining at the waste unit above levels that allow for unlimited use and unrestricted exposure. Estimated costs associated with Alternative S-3 are summarized below.

\begin{tabular}{|c|c|}
\hline Capital Costs & $\$ 3,000,000$ \\
\hline O\&M Costs & $\$ 430,000$ \\
\hline Total Costs & $\$$ \\
\hline
\end{tabular}

Costs for this alternative are provided in Appendix B.

Evaluation of Soil-Based Alternatives Under the General Response of Containment

A direct comparison of Alternatives S-2 and S-3 reveals that both alternatives are potentially effective at protecting human health and the environment. Short-term risk to human health 
would be equivalent under Alternatives $S-2$ and $S-3$ if backfill material is emplaced in the Basin prior to initiating slurry wall or cap construction. Alternatives S-2 and S-3 should not pose any significant health risk to the public.

Although neither Alternative S-2 nor S-3 involves treatment of contaminants or contaminated media, both alternatives would reduce contaminant mobility by decreasing infiltration and subsequent leaching of contaminants. The slurry walls used in Alternative S-3 would be especially effective at reducing contaminant leaching into the subsurface soil and groundwater. Both alternatives meet the remedial action objective of reducing surficial risks to human health and the environment, and reducing the leaching of primary and secondary COCs to unit groundwater.

Alternatives S-2 and S-3 are both readily implementable. Alternative S-3 would require additional considerations for the construction of the slurry wall; however, this proven technology should be a straight-forward process. The time and effort necessary to construct the slurry wall under Alternative S-3 should be offset by the reduction in further contaminant migration in the LAOCB subsurface soil.

Estimated present worth capital cost of Alternative S-3 is more than double the estimated present worth cost of Alternative S-2. The primary difference in cost is attributed to the cost of constructing the slurry wall under Alternative S-3. Both Alternative S-2 and Alternative S-3 will be retained for detailed analysis.

\subsubsection{Alternatives Under the General Response of In-Situ Treatment}

This subsection contains a description, screening, and evaluation of Alternatives S-4 and S-5. 


\section{Alternative S-4: In-Situ Stabilize the LAOCB, Backfill and Cap the LAOCB}

Alternative S-4 involves in-situ grouting the LAOCB soil. Upon completion of in-situ stabilization process, the LAOCB would be backfilled and capped. In-situ grouting can be accomplished by conventional trackhoes and tillers mounted to trackhoes, jet grouting, or augering.

In-situ S/S does not reduce the total mass of COCs and cannot in itself achieve RGOs, it is a proven performance based engineering approach that reduces the mobility of primary and secondary COCs. Based on results of a literature search and a treatability study performed on LAOCB soils, the in-situ S/S reagents are considered effective at reducing the leachability of contaminants. Specifically the various S/S reagent samples (with LAOCB soil) were subjected to toxicity characteristics leaching procedure (TCLP) and the entended American National Standard (ANS) 16.1 procedure to simulate leaching of contaminants over time. Analysis of leaching test performed on LAOCB soil samples amended with $\mathrm{S} / \mathrm{S}$ reagents demonstrated that all of the samples leached $0.41 \%$ and $1.61 \%$ or less of gross alpha and gross beta, respectively (WSRC, 1996c).

Care would be required to minimize the generation of airborne particulate during processing. This could be accomplished by applying dust suppressants, monitoring for airborne particulate, and strict adherence to project work plans. Standing water, if present, could also be used to suppress dust within the Basin. In-situ grouting would begin at one end of the LAOCB and progress outward and/or along the Basin bank until all soil are treated. Once grouting is completed, the remaining capacity of the LAOCB would be backfilled and compacted to grade followed by construction of a cap over the LAOCB as described under Alternative S-2.

Alternative S-4 would be protective of human health and the environment. The cap would function as a physical barrier to prevent direct human exposure to soil-borne contamination and 
would minimize infiltration and subsequent leaching of contamination from unsaturated soil to the groundwater. If properly constructed and maintained, caps can provide effective long-term

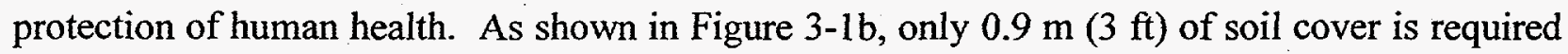
to reduce the annual effective dose from continuous exposure to the ${ }^{137} \mathrm{Cs}$ and ${ }^{60} \mathrm{Co}$ in the Basin by over 99 percent and within regulatory and DOE limits. As demonstrated in the treatability study (WSRC, 1996c), grouting of the contaminated LAOCB soil and sludge would provide an added measure of long-term protection by significantly decreasing the mobility of radionuclides in LAOCB soil. Therefore, Alternative S-4 meets the remedial action objective of reducing surficial risks to human health and the environment, and reducing the leaching of tritium and primary and secondary COCs to unit groundwater.

In-situ stabilization of soil is a relatively straight-forward process; however, due to the increased handling of contaminated media, the potential for elevated short-term risk to the health of remedial workers is increased. Exposure can be minimized and maintained below acceptable levels with the use of proper protective clothing, scheduled monitoring of area radiation and airborne particulate levels, and strict adherence to the project health and safety plan. Short- and long-term risks associated with cap construction are discussed under Alternative S-2.

Alternative S-4 would involve in-situ stabilization which is an active form of treatment thatwould reduce the mobility of contaminants in LAOCB soil. One disadvantage of S/S technologies is that the volume of contaminated media may increase due to the addition of additives such as grout; however, the treated media would be contained within the LAOCB and would not add to the overall volume of waste to be managed. Additionally, the treatability study conducted for this unit indicated very little volume increase for the samples tested (WSRC, 1996c). When used in conjunction with capping, grouting would further reduce contaminant mobility, thereby reducing the inherent risk to the environment and human health. 
In general, in-situ stabilization should be readily implementable. Alternative S-4 would require readily available reagents as determined during the treatability study [(WSRC, 1996c) e.g., Portland cement, bentonite, silicate], and the use of conventional or non-conventional delivery systems. Extensive decontamination of equipment would be required since the process would be conducted in-situ. Numerous qualified contractors are available that can competitively bid on project design and construction. In-situ grouting used in conjunction with capping has been used at other waste sites, and generally does not elicit public concerns. Implementability of cap construction is discussed under Alternative S-2.

Costs associated with Alternative S-4 include the labor and materials needed to grout the LAOCB sludge and soil and construct a cap over the LAOCB. Also included in the costs is the operation and maintenance of the cap for 30 years. These costs include a review of remedy every five years for 30 years as required by the NCP. Five year remedy reviews are required for remedial actions that result in hazardous substances, pollutants, or contaminants remaining at the waste unit above levels that allow for unlimited use and unrestricted exposure. Estimated capital and $O \& M$ costs associated with Alternative S-4 are summarized below.

$\begin{array}{ll}\text { Capital Costs } & \$ 3,150,000 \\ \text { O\&M Costs } & \$ \frac{430,000}{} \\ \text { Total Costs } & \$ 3,580,000\end{array}$

Costs for this alternative are provided in Appendix B.

\section{Alternative S-5: In-Situ Vitrify LAOCB, Backfill and Cap the LAOCB}

Alternative S-5 involves the in-situ vitrification of the LAOCB soil and encapsulating the primary and secondary COCs. In-situ vitrification is an engineering approach that does not reduce the total mass of COCs and can not achieve RGOs. However, the primary goal of In-situ vitrification is to reduce the mobility of primary and secondary COCs. Upon completion of in- 
situ vitrification, the LAOCB would be backfilled and compacted to grade, and the LAOCB would be capped as described under Alternative S-2. Intense heat is generated within the soil matrix until the matrix becomes vitrified. When the heating energy is removed, the molten soil or melt cools into a solid, glassy matrix. Because the process involves intense heating, the efficiency of the vitrification process is reduced as the moisture content of the soil matrix increases. It is for this reason that the $\mathrm{LAOCB}$ waste would be homogenized into a relatively dry mixture prior to the initiation of soil vitrification.

To initiate the melting process, a conductive mixture of flaked graphite and glass frit is typically placed between the electrodes to act as a starter path for the electric circuit. Because SRS soil have been found to be deficient in $\mathrm{CaO}, \mathrm{Na}_{2} \mathrm{O}$, and $\mathrm{K}_{2} \mathrm{O}$, a flux of 5 to $10 \mathrm{wt} \%$ sodium carbonate may be necessary to vitrify LAOCB waste unit soil. Based upon results of bench-scale studies conducted on SRS soil, the soil amendments must be pre-mixed into the soil to the desired treatment depth to ensure adequate penetration of the melt (EPA, 1992). This would involve the in-situ mixing of amendments using conventional earthmoving equipment. A unit-specific treatability study would be conducted to determine amendment requirements specific to LAOCB waste unit soil. To minimize equipment downtime, all soil would be pretreated prior to initiating in-situ vitrification. When pretreatment of soil is completed, the vitrification unit electrodes would be inserted to the desired depth and current passed between the electrodes. The vitrification process would be repeated until all LAOCB soil are processed. At the conclusion of the vitrification process, the remaining capacity of the LAOCB would be backfilled and a cap constructed over the LAOCB as described under Alternative S-2.

Alternative S- 5 would be protective of human health and the environment. The vitrified soil would form a very hard, durable, glassy, solid monolith that would be very resistant to leaching and is considered a permanent remediation solution (Oma, 1994). Residual organic compounds present would be permanently destroyed, volatile compounds would be contained in the off-gas treatment system, and remaining inorganic constituents would be chemically incorporated in the 
resulting monolith. The monolith would be capable of withstanding long-term environmental exposure without effect. In addition, the cap would function as a physical barrier to deter direct human contact with the subsurface monolith, prevent infiltration, and shield the surface from gamma emitting radiation. Therefore, Alternative S-5 meets the remedial action objective of reducing surficial risks to human health and the environment, and reducing the leaching of primary and secondary COCs to unit groundwater.

In-situ vitrification of soil at the LAOCB would be a relatively involved, complex process. A treatability study would be warranted to determine optimum recipe of amending soil, followed by pretreatment of soil. Pretreatment of soil would require unit preparations similar to those discussed under Alternative S-4. Further unit preparation would be required to support the insitu vitrification process. Short-term risk to the health of remedial workers would increase (over Alternative S-3) with the additional handling and processing of contaminated media. The use of protective measures (e.g., barrier soil, dust collection hood, off-gas collection hood), would minimize remedial worker exposure to airborne emissions and maintain their exposure below acceptable levels. Additional safety measures include the use of proper protective clothing, scheduled monitoring of area radiation and airborne particulate levels, and strict adherence to the project health and safety plan. Short- and long-term risks associated with cap construction are discussed under Alternative S-2.

In-situ vitrification would treat contaminated soil, resulting in reduced contaminant mobility and contaminated media volume. Because the contaminants would be permanently immobilized, Alternative S-5 would greatly reduce the inherent risk associated with LAOCB contaminated media. Although in-situ vitrification would reduce the volume of contaminated media (i.e., eliminate pore space in soil matrix), the monolith would remain within the LAOCB and would not lessen the overall volume of waste to be managed. 
Even though in-situ vitrification has undergone extensive testing and is considered potentially feasible for a variety of applications, in-situ vitrification may be considered an innovative technology due to limited application on large-scale projects and limited number of large-scale systems. Two large-scale in-situ vitrification systems have been designed and fabricated. One of the systems was designed for remediating industrial waste sites, and the other system was designed for testing on DOE radioactive and mixed waste sites. The large-scale DOE system is designed to accommodate electrode separations of 3.5 to $5.5 \mathrm{~m} \mathrm{(11.5} \mathrm{to} 18 \mathrm{ft}$ ) and treatment

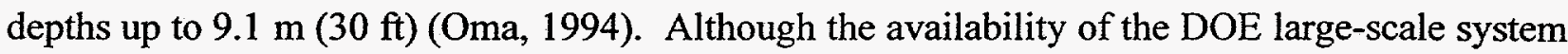
may be limited, in-situ vitrification should be implementable.

Soil amendments (sodium carbonate) should be readily available. Although the rigs needed to deliver the reagents is not considered conventional, there should be a number of contractors available that can procure the required equipment. In addition, conventional earthmoving equipment would be required (e.g., backhoes, dump trucks, bull dozers, compactors) to backfill the LAOCB at the conclusion of in-situ vitrification operations. Only a limited number of qualified contractor are available that can design and construct an in-situ vitrification system for use at a USDOE waste unit (Tixier, 1995). In-situ vitrification used in conjunction with capping would not be expected to elicit great public concerns. Implementability of cap construction is discussed under Alternative S-2.

Costs associated with Alternative S-5 include the labor and materials needed to pretreat soil insitu, vitrify LAOCB soil in-situ, and to construct a cap over the LAOCB. Also included in the costs is the operation and maintenance of the cap for 30 years. These costs include a review of remedy every five years for 30 years as required by the NCP. Five year remedy reviews are required for remedial actions that result in hazardous substances, pollutants, or contaminants remaining at the waste unit above levels that allow for unlimited use and unrestricted exposure. Estimated costs associated with Alternative S-5 are summarized below. 


\begin{tabular}{|c|c|}
\hline Capital Costs & $\$ 6,830,000$ \\
\hline O\&M Costs & $\$ \quad 430,000$ \\
\hline Total Costs & $\Phi, 2$ \\
\hline
\end{tabular}

Costs for this alternative are provided in Appendix B.

Evaluation of Alternatives Under the General Response of In-Situ Treatment

Alternatives S-4 and S-5 are potentially effective at protecting human health and the environment. The short-term risk to human health is greatest under Alternative S-5 since it involves much greater processing of contaminated soil than Alternatives $S-4$. The potential increase in health risks to remedial workers can be maintained within acceptable limits under Alternatives S-4 and S-5 as long as there is strict adherence to the project health and safety plan. Alternatives S-4 and S-5 should not pose significant health risk to the public.

Alternatives S-4 and S-5 all involve treatment of contaminated media that reduces contaminant mobility. Alternative S-5 would most effectively reduce contaminant mobility since contaminants would be chemically and physically bound within the vitrified matrix permanently. In-situ grouting proposed under Alternative S-4 could result in an increase in contaminated media volume, whereas vitrification proposed under Alternative S-5 would reduce contaminated media volume. However, in each case, the net volume of waste to be managed would not be affected because in all cases, it would be the waste contained beneath the cap covering the LAOCB. Both alternative meet the remedial action objective of reducing risks to human health and the environment, and reducing the leaching of primary and secondary COCs to unit groundwater.

Alternative S-4 requires more conventional equipment and manpower and is more easily implemented than Alternative S-5. The time period to implement and complete Alternative S-4 would be short as compared to implementing and completing Alternative S-5. In-situ 
vitrification proposed under Alternative S-5 is more difficult to implement than Alternative S-4 because of the limited availability of an in-situ vitrification unit, the need to pretreat soil using specialty equipment, and the need for a very limited number of highly skilled and trained operators to conduct process operations. The time period required to implement and complete Alternative S-5 would be much greater than Alternative S-4.

Estimated total present worth costs of Alternative S-5 is more than double the estimated total present worth costs associated with Alternative S-4. The differences in present worth costs are attributed to differences in in-situ treatment costs since costs associated with Alternatives S-4 and S-5 all include long-term maintenance of a cap covering the LAOCB.

Although vitrification of soil would be much more effective in immobilizing soil-borne contamination than treating using a $\mathrm{S} / \mathrm{S}$ technology, the apparent need to pretreat the soil prior to vitrification significantly lessens the feasibility of Alternative S-5 as an in-situ treatment alternative. Based upon bench-scale testing of SRS soil, LAOCB soil will likely require pretreatment for the addition sodium carbonate. Pretreating of the soil would be very costly and would significantly prolong the time required to complete remedial actions at the LAOCB waste unit. In addition, Alternative S-5 is clearly less implementable and much more costly than Alternative S-4. Alternative S-5 will therefore be rejected from further consideration on the basis of implementability and cost. Alternative S-5 will not be retained for detailed analysis.

\subsubsection{Alternatives Under the General Response of Ex-Situ Treatment}

Following is a description, screening, and evaluation of Alternatives S-6 and S-7. 
Alternative S-6: Excavate LAOCB Soil, Stabilize Soil Ex-Situ, Place into LAOCB, Backfill and Cap LAOCB

As described for Alternative S-4, Alternative S-6 is a proven engineering approach that does not reduce the total mass of COCs and can therefore not achieve RGOs. However, the primary goal of this alternative is to minimize the mobility of primary and secondary COCs. Alternative S-6 involves the excavation and ex-situ stabilization of LAOCB soil. Upon completion of ex-situ stabilization, treated soil would be placed back inside the LAOCB, the LAOCB would be backfilled and compacted to grade, and the LAOCB would be capped as described under Alternative S-2. The equipment needed to conduct ex-situ grouting of soil would likely include conventional mixing equipment (e.g., pug mill, mixers) to S/S the soil according to the results of the S/S treatability study which indicates that LAOCB soil could effectively be treated using conventional reagents (WSRC, 1996c).

Prior to excavation, soil and subsurface soil would be pre-treated to ensure optimal waste handling characteristics. Subsequently, the LAOCB soil would be excavated using a trackhoe. The trackhoe would start at one end of the Basin and would gradually progress along the edges

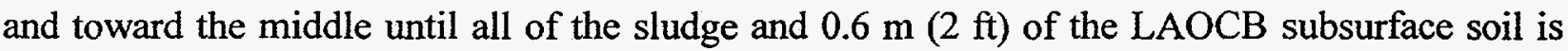
removed. Pre-treated excavated soil would be staged at a protective area adjacent to the Basin prior to processing. The staged soil would be mixed with the $S / S$ reagents at predetermined ratios and the soil would be placed back into the Basin. Standing water could be used to hydrate $\mathrm{S} / \mathrm{S}$ reagents as needed, but may require removal and proper disposal if present in excess. As discussed under Alternative S-4, results of the treatability study suggests that the use of portland cement, bentonite, and silicates are effective at reducing the mobility of radionuclides identified in the $\mathrm{LAOCB}$.

Once excavation and grouting activities are complete, the remaining capacity of the LAOCB would be backfilled and compacted to grade, followed by the construction of a cap over the 
LAOCB as described under Alternative S-2. If properly constructed and maintained, caps can provide effective long-term protection of human health. As shown in Figure 3-1b, only $0.9 \mathrm{~m}$ (3 $\mathrm{ft}$ ) of soil cover is required to reduce the annual effective dose from continuous exposure to the ${ }^{137} \mathrm{Cs}$ and ${ }^{60} \mathrm{Co}$ in the Basin by over 99 percent and within regulatory and DOE limits. Grouting of the contaminated LAOCB sludge/soil would provide an added measure of long-term protection by significantly decreasing the mobility of primary and secondary COCs in LAOCB soil. Therefore, Alternative S-6 meets the remedial action objective of reducing risks to human health and the environment, and reducing the leaching of primary and secondary COCs to unit groundwater.

Care would be required to minimize the generation of airborne particulate during processing. This could be accomplished by applying dust suppressants, monitoring for airborne particulate, and strict adherence to project work plans. Standing water, if present, would minimize the generation of dust from the Basin.

Alternative S-6 would be protective of human health and the environment. The cap would function as a physical barrier to prevent direct human exposure to soil-borne contamination and would minimize infiltration and subsequent leaching of contamination from unsaturated soil to the groundwater. If properly constructed and maintained, caps can provide effective long-term protection of human health and the environment. Grouting of the LAOCB sludge and subsurface soil would provide an added measure of long-term protection by immobilizing the primary and secondary COCs associated with LAOCB soil.

Ex-situ stabilization of soil is a relatively straight-forward process; however, due to the increased handling of contaminated media, there would be a potential for elevated short-term health risk to remedial workers. With the proper use of protective clothing, scheduled monitoring of area radiation and airborne particulate levels, and strict adherence to the project health and safety plan, remedial worker exposure can be minimized and maintained below acceptable levels. Ex- 
situ grouting should not pose any significant health risk to the public. Short- and long-term risks associated with cap construction are discussed under Alternative S-2.

Alternative S-6 would involve ex-situ grouting which is an active form of treatment that would reduce the mobility of contaminants in LAOCB soil. Ex-situ stabilization could increase the volume of contaminated media; however, the treated media would be contained within the LAOCB and would not add to the overall volume of waste to be managed. When used in conjunction with capping, grouting would greatly reduce contaminant mobility, thereby reducing the inherent risk to the environment and human health.

Ex-situ stabilization should be readily implementable. Results of the unit-specific $\mathrm{S} / \mathrm{S}$ treatability testing indicate that Alternative S- 6 would require readily available reagents such as Portland cement, bentonite, and silicate (WSRC, 1996c). Conventional earthmoving equipment (e.g., trackhoes, backhoes, dump trucks, bull dozers, compactors) and mixing equipment also are available. Extensive decontamination of equipment would be required since the process would involve significant materials handling and transport. Numerous qualified contractors should be available that could competitively bid on project design and construction. Ex-situ grouting used in conjunction with capping has been used at other waste sites and generally does not elicit public concerns. Implementability of cap construction is discussed under Alternative S-2.

Costs associated with Alternative S-6 include the labor and materials needed to pre-treat the soil prior to excavation for waste handling purposes, excavation and treatment of the LAOCB sludge and subsurface soil, and to construct a cap over the LAOCB. Also included in the costs is the operation and maintenance of the cap for 30 years. These costs include a review of remedy every five years for 30 years as required by the NCP. Five year remedy reviews are required for remedial actions that result in hazardous substances, pollutants, or contaminants remaining at the waste unit above levels that allow for unlimited use and unrestricted exposure. Estimated costs associated with Alternative S-6 are summarized below. 


$\begin{array}{ll}\text { Capital Costs } & \$ 3,940,000 \\ \text { O\&M Costs } & \$ 4430,000 \\ \text { Total Costs } & \$ 4,370,000\end{array}$

Costs for this alternative are provided in Appendix B.

Alternative S-7: Excavate LAOCB Soil, Ex-Situ Vitrify Soil, Place Treated Soil into LAOCB, Backfill and Cap LAOCB

Alternative S-7 involves the excavation and ex-situ vitrification of the sludge and top $0.46 \mathrm{~m}$ (1.5 ft) of LAOCB sludge and subsurface soil. Ex-situ vitrification is an engineering approach that does not reduce the total mass of COCs and can not achieve RGOs. However, the primary goal of this alternative is to reduce the leaching of primary and secondary COCs into unit groundwater. Upon completion of ex-situ vitrification, the vitrified soil would be placed back into the LAOCB. The LAOCB would be backfilled and compacted to grade, and the LAOCB would be capped as described under Alternative S-2.

Ex-situ vitrification of soil involves the vitrification of contaminated media in a vitrification unit separate from where the contaminated media is located. Initially, an ex-situ vitrification unitwould be specifically designed to accommodate LAOCB contaminated media. The minimum time required to design and construct a Terra-Vit portable ex-situ vitrification unit would be at least one year (Tixier, 1995). Treatability testing would be conducted as required to support unit design. When designed, the vitrification unit and support equipment would be mobilized to the LAOCB waste unit, constructed and tested.

Prior to excavation, soil and subsoil would be treated to ensure optimal waste handling characteristics. Subsequently, a trackhoe would be used to excavate LAOCB soil. The trackhoe would start at one end of the Basin and would gradually progress along the edges and toward the 
middle of the Basin until all of the sludge and subsurface soil to $0.6 \mathrm{~m}(2 \mathrm{ft})$ is removed. Pretreated excavated soil would be staged at a protective area adjacent to the Basin prior to processing.

Because SRS soil have been found to be deficient in the amount of $\mathrm{CaO}, \mathrm{Na}_{2} \mathrm{O}$, and $\mathrm{K}_{2} \mathrm{O}$, it may be necessary to amend the soil with a flux of five to ten percent by weight sodium carbonate in order to vitrify LAOCB waste unit soil (EPA, 1992). A unit-specific treatability study would be conducted to determine if soil amendments are required.

LAOCB soil will be excavated, amended, and processed through the vitrification unit with the vitrified soil being returned to the Basin. The vitrification process would continue until all LAOCB soil are processed. At the conclusion of the vitrification process, the remaining capacity of the LAOCB would be backfilled and compacted to grade, and a cap would be constructed over the LAOCB as described under Alternative S-2.

Alternative S-7 would be protective of human health and the environment. The vitrified soil would form a very hard, durable, glassy, solid monolith that would be very resistant to leaching and is considered a permanent remediation solution (Oma, 1994). Organic compounds present would be permanently destroyed, volatile compounds would be contained in the off-gas treatment system and remaining inorganic constituents would be chemically incorporated in the resulting melt. The melt should be capable of withstanding long-term environmental exposure without effect. In addition, the cap would function as a physical barrier to deter direct human contact with the subsurface monolith. Therefore, Alternative S-7 meets the remedial action objective of reducing risks to human health and the environment and reducing the leaching of primary and secondary COCs to unit groundwater.

Ex-situ vitrification of soil at the LAOCB would be a relatively involved, complex process. Vitrification of LAOCB soil would be preceded by design, construction, and testing of an ex-situ 
vitrification unit. In addition, a treatability study would be warranted to determine design requirements and the optimum recipe for amending soil. Extensive unit preparation would also be required to support the ex-situ vitrification process.

Short-term risk to the health of remedial workers would exist due to the extensive handling and processing of contaminated media. The use of protective measures (e.g., barrier soil, off-gas collection hood), would minimize remedial worker exposure to airborne emissions and maintain their exposure below acceptable levels. Additional safety measures include the use of proper protective clothing, scheduled monitoring of area radiation and airborne particulate levels, and strict adherence to the project health and safety plan. Ex-situ vitrification should not pose any significant health risk to the public. Short- and long-term risks associated with cap construction are discussed under Alternative S-2.

Ex-situ vitrification would treat contaminated soil, resulting in significantly reduced contaminant mobility and contaminated media volume. Alternative S-7 would greatly reduce the inherent risk associated with LAOCB contaminated media since the contaminants would be permanently immobilized within the glass matrix. Although ex-situ vitrification would reduce the volume of contaminated media, the vitrified product would remain within the LAOCB and would not lessen the overall volume of waste to be managed.

Ex-situ vitrification has undergone extensive testing and is frequently used for treating high-level radioactive waste; however, on-unit vitrification of low-level or mixed waste using a portable melter may be considered an innovative technology since there has been limited application of ex-situ vitrification on large-scale projects and there are no large-scale systems available for immediate use. In addition, only a limited number of contractors would be available to design and construct a Terra-Vit melter (Tixier, 1995). Despite these limitations, Alternative S-7 should be implementable. Ex-situ vitrification using a portable Terra-Vit melter has been demonstrated on a comparable project (Chapman, 1993). Although ex-situ vitrification is a relatively 
innovative technology, it would not be expected to elicit great public concerns. Implementability of cap construction is discussed under Alternative S-2.

Costs associated with Alternative S-7 include the labor and materials needed to design and construct a portable ex-situ vitrification system, to pre-treat the soil and subsoil for waste handling purposes, to evacuate and vitrify the soil, and to construct a cap over the LAOCB. Also included in the costs is the operation and maintenance of the cap for 30 years. These costs include a review of remedy every five years for 30 years as required by the NCP. Estimated costs associated with Alternative S-7 are summarized below.

\begin{tabular}{|c|c|}
\hline Capital Costs & $\$ 8,270,000$ \\
\hline O\&M Costs & 430,000 \\
\hline Total Costs & \\
\hline
\end{tabular}

Costs for this alternative are provided in Appendix B.

\section{Evaluation of Alternatives Under the General Response of Ex-Situ Treatment}

Alternatives S-6 and S-7 are potentially effective at protecting human health and the environment. The short-term risk to human health is greatest under Alternative S-7 since it involves much greater processing of contaminated soil than does Alternative S-6. The potential increase in health risks to remedial workers can be maintained within acceptable limits under these alternatives as long as there is strict adherence to the project health and safety plan. Alternatives S-6 and S-7 should not pose any significant health risk to the public.

Alternatives S-6 and S-7 involve treatment of contaminated media that reduces contaminant mobility. In terms of effectiveness, Alternative S-7 would most effectively reduce contaminant mobility since it would chemically and physically immobilize contaminants permanently within a vitrified matrix. Both alternative meets the remedial action objective of reducing risks to 
human health and the environment and reducing the leaching of primary and secondary COCs to unit groundwater.

Ex-situ grouting proposed under Alternative S-6 would result in an increase in contaminated media volume, whereas ex-situ vitrification proposed under Alternative S-7 would reduce contaminated media volume. However, because there is excess capacity in the LAOCB and the waste will have been substantially immobilized, an increase in volume should not be a problem.

Alternatives S-6 and S-7 would require conventional excavation equipment and manpower. The time to implement and complete the remedial alternative would be shortest under Alternative S6. Ex-situ vitrification proposed under Alternative $S-7$ is the most difficult technology to implement because a portable vitrification unit must first be designed, constructed, and tested before it can be used to treat soil. In addition, operation of ex-situ vitrification equipment would require the services of a very limited number of highly skilled and trained operators to conduct process operations. The time required to implement and complete Alternative S-7 would be much greater than for Alternative S-6.

The estimated present worth cost to treat LAOCB waste unit soil by vitrification are more than double the cost of treating the waste by grouting. This disparity in costs is due to the high cost of vitrification system design, construction, and operation.

Of the two alternatives considered for the general response of ex-situ treatment, Alternative S-6 will be retained for detailed analysis, and Alternative S-7 will be rejected. Although Alternative S-7 is implementable, it requires more waste handling, which increases short-term health risks to remedial workers, and is cost prohibitive. Therefore, Alternative S-7 will be rejected on the basis of effectiveness and cost and Alternative S- 6 will be retained for detailed analysis. 


\subsubsection{Alternatives Under the General Response of Off-Unit Disposal}

This subsection contains a description, screening, and evaluation of Alternatives S-8, S-9, and S10.

Alternative S-8: Excavate LAOCB, Dispose of Soil at the Pre-Conceptual SRS Soil/Debris Consolidation Facility, Backfill LAOCB

Alternative S-8 involves the excavation and disposal of LAOCB at a conceptual SRS Soil Consolidation Facility. The Soil Consolidation Facility is being discussed with agencies as an alternative strategý for off-unit disposal. The Soil Consolidation Facility would be a central disposal facility for SRS generated radiologically contaminated wastes.

The SRS Soil Consolidation Facility would require siting, design, and construction prior to considering the disposal of wastes from the LAOCB. Siting, design, and construction is likely to require over three years for implementation. This time frame is expected to be beyond issuance of the Statement of Basis and ROD and beyond the Corrective Measure Implementation/ Remedial Action schedule for remediation of the LAOCB. Costs cannot be estimated for disposal at the Soil Consolidation Facility since it has not been sited, designed, or constructed. and waste acceptance criteria have not been established. Disposal at the pre-conceptual Soil Consolidation Facility is temporarily rejected; however, should the facility become operational in the future, disposal of LAOCB wastes could be re-evaluated.

\section{Alternative S-9: Excavate LAOCB, Dispose of Soil at the NTS Facility, Backfill LAOCB}

Alternative S-9 involves the excavation and off-site disposal of LAOCB soil. Soil and subsoil would first be treated to ensure optimal waste handling characteristics. Following pre=treatment, a trackhoe would be used to excavate LAOCB waste unit soil. Post-excavation treatment may 
further be required for waste handling purposes, and packaging and disposal requirements. Treated excavated soil would be placed directly onto lined haul trucks for transport from the waste unit. Prior to being transported from the LAOCB waste unit, representative soil samples would be collected from the excavated soil for characterization. After characterization, the excavated soil would be transported to the NTS facility near Mercury, Nevada, directly by truck or intermodal by both rail and truck. During excavation, confirmation soil samples would be collected and analyzed to verify that all soil above a concentration-based cleanup limit were removed. Upon completion of the excavation and off-site disposal activities, the LAOCB would be backfilled to prevent the accumulation of water and for health and safety purposes.

Alternative S-9 would be protective of human health and the environment. Alternative S-9 would permanently remove all soil-borne contamination known to be present at the LAOCB waste unit. Therefore, Alternative S-9 meets all remedial action objectives through achieving RGOs, reducing risks to human health and the environment, and reducing the leaching of primary and secondary COCs to unit groundwater. Short-term risk to the health of remedial workers would exist due to the extensive handling and processing of contaminated media. With the use of protective measures such as the use of proper protective clothing and equipment, scheduled monitoring of area for radiation and airborne particulate levels, and strict adherence to the project health and safety plan, remedial worker exposure can be maintained within acceptable levels. In addition, there would also be short-term risk to the public during transport of the contaminated soil from SRS to the NTS facility $(3,500 \mathrm{~km} ; 2,200 \mathrm{mi})$. If the waste is transported via railcar and then transferred to a truck for final transport to the NTS facility, risk of worker and public exposure is increased.

Alternative S-9 would not involve any form of treatment that would result in a reduction of contaminant toxicity, mobility, or volume. 
Off-site disposal of low-level radioactive or mixed wastes is implementable; however, it requires extensive waste handling. The NTS facility is certified to accept low-level radioactive wastes and mixed wastes from various sources including DOE facilities and has available capacity to accept the waste. A number of transporters are available that are certified and qualified to transport low-level radioactive and mixed wastes. There is a potential for public resistance to Alternative S-9 since there is potential risk to the public during waste transport. Assuming that gondolas and haul truck capacities are $34 \mathrm{~m}^{3}\left(1,200 \mathrm{ft}^{3} ; 44 \mathrm{yd}^{3}\right)$ and $13.8 \mathrm{~m}^{3}\left(486 \mathrm{ft}^{3} ; 18 \mathrm{yd}^{3}\right)$, respectively, approximately 23 rail gondolas of contaminated soil or 56 truckloads would be transferred to Nevada from SRS.

Costs associated with Alternative S-9 include the labor and materials needed to pre-treat soil and subsoil for waste handling purposes, to excavate the wastes, to treat the wastes following excavation for packaging and disposal requirements, to transport the wastes, and to dispose of LAOCB soil. A review of remedy would not be required for $L A O C B$ soil under this alternative since the unit soil would contain no hazardous substances, pollutants, or contaminants above concentration-based remediation goals. In addition, there are no long-term operation and maintenance costs associated with Alternative S-9 since it would not involve the construction of a cap. Estimated costs associated with Alternative S-9 are summarized below.

$\begin{array}{lr}\text { Capital Costs } & \$ 9,100,000 \\ \text { O\&M Costs } & \$ \frac{0}{100,000} \\ \text { Total Costs } & \$ 9,100,00\end{array}$

Costs for this alternative are provided in Appendix B. 


\section{Alternative S-10: Excavate LAOCB, Dispose of Soil at the Envirocare Facility, Backfill} $\underline{\mathrm{LAOCB}}$

Alternative S-10 is generally the same as Alternative S-9 with the exception of the location of the disposal facility and the costs for disposal.

The Envirocare facility is certified to accept low-level radioactive wastes and mixed wastes from various sources including DOE facilities and has available capacity to accept $4.4 \mathrm{E} 4 \mathrm{~m}^{3}(1.5 \mathrm{E} 6$ $\mathrm{ft}^{3} ; 5.5 \mathrm{E} 4 \mathrm{yd}^{3}$ ) of material. A number of transporters are available that are certified and qualified to transport low-level radioactive and mixed wastes. There is a potential for public resistance to Alternative S-10 since there is potential risk to the public during waste transport. Assuming that gondolas and haul truck capacities are $34 \mathrm{~m}^{3} \cdot\left(1,200 \mathrm{ft}^{3} ; 44 \mathrm{yd}^{3}\right)$ and $13.8 \mathrm{~m}^{3}\left(486 \mathrm{ft}^{3} ; 18 \mathrm{yd}^{3}\right)$, respectively, approximately 23 rail gondolas or 56 truckloads of contaminated soil would be transferred to Utah from the SRS.

Costs associated with Alternative S-10 include the labor and materials needed to excavate, transport and dispose of LAOCB soil. A review of remedy would not be required for LAOCB soil under this alternative since the unit soil would contain no hazardous substances, pollutants, or contaminants above concentration-based remediation goals. In addition, there are no longterm operation and maintenance costs associated with Alternative $S-10$ since it would not involve the construction of a cap. Estimated costs associated with Alternative S-10 are summarized below.

\begin{tabular}{|c|c|}
\hline Capital Costs & $\$ 13,000,000$ \\
\hline O\&M Costs & $\$$ \\
\hline Total Costs & $\$ 13,000,000$ \\
\hline
\end{tabular}

Costs for this alternative are provided in Appendix B. 
Evaluation of Alternatives Under the General Response of Off-Unit Disposal

Off-unit disposal Alternatives S-8, S-9, and S-10 are potentially effective at protecting human health and the environment. The potential increase in health risks to remedial workers can be maintained within acceptable limits under these alternatives as long as there is strict adherence to the project health and safety plan. The short-term risk to human health is greater under Alternatives S-9 and S-10 because they would involve transport of contaminated soil $3,500 \mathrm{~km}$ $(2,200 \mathrm{mi})$ and $3,200 \mathrm{~km}(2,000 \mathrm{mi})$ to the NTS and Envirocare facilities, respectively, as opposed to disposing of the soil on site under Alternative S-8. The greatest risk to the public would most likely be in the form of traffic-related accidents.

Estimated present worth costs of Alternatives S-9 and S-10 are similar with Alternative S-10 costs being higher. The difference in present worth costs are attributed to higher disposal costs associated with the Envirocare facility. Costs associated with Alternative S-8 cannot currently be determined since the Soil Consolidation Facility has not been sited, designed, or constructed and no waste acceptance criteria have been established.

Alternatives S-8, S-9, and S-10 are all effective at protecting human health and the environment, and are implementable. All three alternatives meet all remedial action objectives through . achieving RGOs, reducing risks to human health and the environment, and reducing the leaching of primary and secondary COCs to unit groundwater. While all three alternatives would permanently remove contaminated soil from the LAOCB waste unit, Alternatives S-9 and S-10 involve an increased risk to human health and the possible opposition of the public to longdistance transport to the NTS or Envirocare facilities. Because Alternative S-10 involves significantly greater cost than Alternative S-9, it will be rejected from further consideration on the basis of cost. Alternative S-8 cannot be evaluated until the SRS Soil Consolidation Facility is operational. Alternative S-8 will be temporarily rejected from further consideration on the basis of implementability. Should the SRS Soil Consolidation Facility become operational, 
disposal of LAOCB wastes at the facility could be re-evaluated. Alternative S-9 will be retained for detailed analysis.

\subsubsection{Summary of LAOCB Alternative Screening}

In this section, the results of screening potential remedial alternatives will be provided. Table 3-1 summarizes each alternative, its status, and the reasons for its status. The only No Action alternative was retained. Both containment alternatives were retained. Of the two in-situ treatment alternatives, in-situ stabilization (Alternative S-4) was retained and in-situ vitrification (Alternative S-5) was rejected on the basis of implementability and cost. Of the two ex-situ treatment alternatives, ex-situ stabilization (Alternative S-6) was retained and Alternative S-7 (ex-situ vitrification) was rejected on the basis of effectiveness and cost. Of the three off-site disposal alternatives, disposal at the SRS Soil Consolidation Facility (Alternative S-8) was rejected on the basis of implementability. Alternative S-9 (disposal at the NTS facility) was retained. Alternative S-10 (disposal at the Envirocare facility) was rejected on the basis of cost. The retained LAOCB remedial alternatives will undergo detailed analysis in Section 4.0.

The retained alternatives were renumbered in sequential order for further discussion in Section 4. The retained alternatives are identified as follows in the remainder of this document:

Alternative S-1: $\quad$ No Action

Alternative S-2: Capping

Alternative S-3: $\quad$ Slurry Cut-Off Wall \& Capping

Alternative S-4: In-Situ Stabilization \& Capping

Alternative S-5: $\quad$ Ex-Situ Stabilization \& Capping

Alternative S-6: $\quad$ Excavation \& Disposal at the NTS Facility 


\subsection{Development and Evaluation of Remedial Alternatives for LAOCB Pipelines}

In Section 2.6.2, remedial technologies were listed as potentially applicable management methods for the pipelines. In Section 3.2.1, these technologies are assembled into functional alternatives which address unit-specific factors. The resulting alternatives are screened in Section 3.2.2. Those alternatives that are retained from screening are subjected to detailed analysis in Section 4.0 .

\subsubsection{Development of Remedial Alternatives}

Potential remedial alternatives have been developed to address the LAOCB pipelines. The NCP requires that a range of alternatives that include treatment to reduce toxicity, mobility, or volume of contaminants be developed and evaluated. The range of alternatives developed includes alternatives which immobilize residual contaminants and to the maximum extent feasible and eliminate or minimize the need for long-term management. Alternatives have also been developed which involve no treatment, but which provide protection to human health and the environment by preventing or controlling exposure to the contaminants through engineered or institutional controls. As required by the NCP, the No Action alternative is provided as a baseline for comparison.

Alternative P-1: No Action

Rationale: $\quad$ No evidence of radionuclide migration from the pipelines to surrounding soils and/or groundwater was identified during the RFI/RI. The pipeline may be retarding the migration potential of radionuclides associated with the pipeline. Additionally, the No Action alternative is required by the NCP. 
Alternative P-2: Institutional/Administrative Controls

Rationale: No evidence of radionuclide migration from the pipelines to surrounding soils and/or groundwater was identified during the RFI/RI. The pipeline may be retarding the migration potential of radionuclides associated with the pipeline. Alternative P-2 would also provide institutional and administrative controls to minimize human access to the pipeline areas, thereby reducing potential future human exposure to contaminants.

\section{Alternative P-3: Capping}

Rationale: Alternative P-3 would involve capping the pipeline areas which would minimize infiltration into and subsequent leaching from the pipelines. Capping would also decrease radioactive exposure from the pipelines to humans and wildlife at the ground surface and would deter access to the pipelines. Alternative P-3 offers no reduction in contaminant toxicity, mobility, or volume.

Alternative P-4: In-Situ S/S

Rationale: Alternative P-4 would involve the injection of grout into the pipelines to immobilize contaminants. Alternative P-4 would immobilize contaminants and further reduce leaching from the pipelines.

Alternative P-5: In-Situ High Pressure Wash and In-Situ S/S

Rationale: Alternative P-5 would involve high pressure washing the LAOCB pipelines from the L-Area Hot Shop (LAHS) end of the pipelines, thereby limiting worker exposure to pipeline contaminants and moving wash water to the LAOCB (prior to remediation of the LAOCB). The pipelines would then be grouted to immobilize any residual contamination to reduce contaminant migration. 
Alternative P-6: In-situ S/S, Excavation, \& Disposal in the LAOCB

Rationale: Alternative P-6 would involve grouting the LAOCB pipelines and subsequently excavating and disposing of the pipelines in the LAOCB. Once inside the LAOCB, in-situ $\mathrm{S} / \mathrm{S}$ will be initiated to encapsulate the pipe in the LAOCB. The pipeline areas would be backfilled and available for unrestricted use. Alternative P-6 would remove contamination from the pipeline areas and reduce the mobility through treatment. However, the toxicity and volume would not be reduced through treatment.

Alternative P-7: In-Situ S/S, Excavation \& Disposal at the Nevada Test Site Facility Rationale: Alternative P-7 would involve grouting the LAOCB pipelines and subsequently excavating and disposing of the pipelines at the NTS facility. The pipeline areas would be backfilled and should be available for future use. Disposal at the NTS would require a $3,500 \mathrm{~km}$ (2,200 miles) transport to the facility and would not involve any treatment to reduce the toxicity, mobility, or volume of contaminants.

\subsubsection{Screening of Remedial Alternatives}

In this section, remedial alternatives developed in Section 3.2.1 undergo a screening evaluation. Alternatives are screened based on the three criteria presented in Section 3.1.2, effectiveness, implementability, and cost. Alternatives retained after screening are subjected to a detailed evaluation in Section 4.0. 


\subsubsection{Alternatives Under the General Response of No Action}

Only one alternative is categorized under the general response of No Action. Following is a description, screening, and evaluation of Alternative P-1.

\section{Alternative P-1: $\quad$ No Action}

Under this alternative, no remedial efforts would be taken to remove, treat, or otherwise affect the toxicity, mobility, or volume of contaminants. Currently, institutional controls are in place that limit access to SRS. These controls would likely be continued in the foreseeable future, but cannot be guaranteed. These controls include continuously manned barricades at primary roads that enter SRS, an exclusion fence around SRS except along the Savannah River, and signs posted against trespassing. Under the No Action alternative, radioactive decay would provide a reduction in radioisotopes present in the pipelines. However, since the specific radioisotopes present in the pipelines have not been identified or qualified, the time required for the radioisotopes to decay within acceptable levels cannot be estimated.

Although institutional controls are likely to be maintained at SRS, their existence cannot be guaranteed. Without institutional controls to prohibit access to the pipeline areas, the potentialexists for human or wildlife intrusion and subsequent exposure to the pipelines. Furthermore, based on the shallow depth of the pipeline [within $0.91 \mathrm{~m}(3 \mathrm{ft})$ of the ground surface], it's relatively high radioactivity level [approximately 300,000 disintegrations per minute (dpm) on internal surface], the age ( $>30$ years) and material of the pipe (cast iron which could degrade over time and release radioisotopes), Alternative P-1 (No Action) would not be protective of human health or the environment. 
The No Action alternative would not require construction, specialized equipment, or technical specialists and could, therefore, be implemented immediately. If implemented, No Action could prompt public concern, depending on the contamination present as a result of the pipelines.

The only costs associated with the No Action alternative would be a review of remedy every five years for 30 years as required by the NCP. Five year remedy reviews are required for remedial actions that result in hazardous substances, pollutants, or contaminants remaining at the waste unit above levels that allow for unlimited use and unrestricted exposure. No long-term operation and maintenance costs would be associated with the No Action alternative. The remedy review costs for the pipelines would be included in that of the LAOCB, dependent upon the remedial action selected for the LAOCB. Costs for the remedy review for the entire OU are $\$ 280,000$.

\section{Evaluation of Alternative P-1}

The risk to human health from exposure to the pipelines is unknown. Since the pipelines are 0.9 $\mathrm{m}$ to $1.2 \mathrm{~m}$ ( 3 to $4 \mathrm{ft}$ ) below ground surface, external radiation exposure from this potential source at the surface would be minimal. However, prevention of future exposure for hypothetical residential scenarios through excavation cannot be guaranteed under the No Action alternative. In addition, since future failure of pipeline integrity could result in the release of radionuclides to the environment, Alternative P-1 is not considered protective of human health or the environment.

The NCP requires that the No Action alternative be retained as a baseline alternative against which all other alternatives can be compared. Therefore, Alternative P-1 is retained for detailed analysis. 


\subsubsection{Alternatives Under the General Response of Institutional/Administrative Controls}

One alternative is categorized under the general response of institutional/administrative controls. Following is a description, screening, and evaluation of Alternative P-2.

\section{Alternative P-2: $\quad$ Institutional/Administrative Controls}

Under Alternative P-2, radionuclides inside the pipeline have not been speciated and the activities of the individual radionuclides associated with the internal surface of the pipeline have not been determined. Therefore, it is not known how much time is required for the radionuclides to decay to acceptable levels. DOE intends to maintain institutional control, which includes monitoring primary road entrances, an exclusion fence around the SRS facility except for along the Savannah River, and signs posting the government ownership of the property, of SRS for a minimum of 100 years.

Based on the shallow depth of the pipeline [(within $0.91 \mathrm{~m}(3 \mathrm{ft})$ of the ground surface]), it's relatively high radioactivity level (approximately $300,000 \mathrm{dpm}$ on internal surface), the age ( $>30$ years) and material or the pipe (cast iron which could degrade over time and release radioisotopes), Alternative P-2 (institutional controls) would not be protective of human health or the environment.

Implementation of this alternative would include posting of signs around the pipelines designating the area as a "controlled area" (CA). Current institutional controls such as manning entrances and perimeter fencing would also be maintained as part of general SRS maintenance until the institutional control period for the site is complete. Implementation of this alternative would, therefore, be relatively simple. 
Costs associated with Alternative P-2 would include posting of signs around the pipeline areas and inspection and maintenance of the signs. A review of remedy would be required every five years for 30 years. The review of remedy costs $(\$ 280,000)$ would be included in that of the LAOCB OU, dependent upon the remedy selected for the LAOCB. Estimated costs associated with Alternative P-2 are summarized below. Costs for this alternative are provided in Appendix B.

\section{Capital Costs $\$ 3,400$ \\ O\&M Costs $\$ \$ 4,600$ \\ Total Costs $\$ 8,000$}

Evaluation of Alternative P-2

Although existing information indicates that the pipeline has prevented migration of radionuclides to the surrounding environment, future failure of pipeline integrity could result in the release of radionuclides to the environment. Therefore, Alternative P-2 is not considered protective of human health or the environment and is not retained for detailed analysis.

\subsubsection{Alternatives Under the General Response of Containment}

One alternative is categorized under the general response of containment. Following is a description, screening, and evaluation of Alternative P-3.

\section{Alternative P-3: $\quad$ Capping}

Under Alternative P-3, the pipeline areas would be capped such that infiltration that could contact the pipelines is minimized. Capping would provide additional protection against migration of contaminants, protection from radiation at the surface, and intrusion by humans and wildlife. A schematic of the proposed cap for the pipeline areas is provided in Figure 3-2b. 
Alternative P-3 would be protective of human health and the environment. Based on current knowledge of the pipeline areas (reviewed in Section 3.2.2.1), the pipelines are not a source of radionuclide contamination in surrounding soils and/or groundwater. Therefore, if infiltration and human intrusion into the pipelines is prevented, the alternative would be effective at protecting human health and the environment. This alternative meets the remedial action objective of reducing the leaching of primary and secondary COCs to unit groundwater.

Implementation of this alternative would include capping the pipeline areas. Capping would involve conventional materials and equipment and would not require any waste handling. Implementation of this alternative would, therefore, be relatively simple.

Costs associated with Alternative P-3 would include capping of the two pipeline areas. A review of remedy would be required every five years for 30 years. The review of remedy costs $(\$ 280,000)$ would be included in that of the LAOCB OU, dependent upon the remedial action selected for the LAOCB. Estimated costs associated with Alternative P-3 are summarized below. Costs for this alternative are provided in Appendix B.

\section{Capital Costs $\$ 560,000$ \\ O\&M Costs $\$ \$ 170,000$ \\ Total Costs $\$ 730,000$}

Evaluation of Alternative $P-3$

Capping should be effective at protecting human health and the environment and meeting the remedial action objective. This alternative is also implementable; however, costs appear to be excessive in relation to other alternatives. Alternative P-3 is retained for detailed analysis. 


\subsubsection{Alternatives Under the General Response of In-Situ Treatment}

Two alternatives are categorized under the general response of in-situ treatment, Alternative P-4, in-situ S/S, and Alternative P-5, high pressure wash and in-situ S/S. Following are descriptions, screenings, and evaluations of Alternatives P-4 and P-5.

\section{Alternative P-4: $\quad$ In-Situ $S / S$}

Under Alternative P-4, the pipelines would be filled with grout from the LAHS end of the pipeline. Grouting would immobilize radionuclide contamination within each pipeline; however, if any cracks or weak areas exist in the pipeline, pressurized grout could enlarge these cracks and allow grout and contamination to escape the pipeline. This combined with the shallow depth of

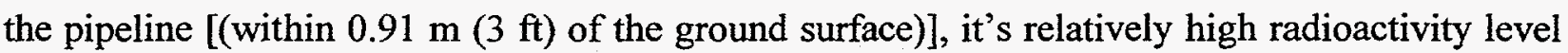
(approximately $300,000 \mathrm{dpm}$ on the internal surface), the age ( $>30$ years) and material for the pipe (cast iron which could degrade over time and release radioisotopes), Alternative P-4 would not be considered protective of human health or the environment.

Implementation of this alternative would include grouting the pipelines and leaving them inplace. In-situ S/S would require general grouting equipment (grout pumps, and grout mixers) that. should be readily available. Exposure to contaminants can be minimized and maintained below acceptable levels with the use of proper protective clothing, scheduled monitoring of area radiation and airborne particulate levels, and strict adherence to the project health and safety plan.

Costs associated with Alternative P-4 would include materials \& equipment required for grouting the two pipelines, plus continued monitoring of the interior surface of the pipelines. A review of remedy would be required every five years for 30 years. The review of remedy costs $(\$ 280,000)$ would be included in that for the LAOCB OU, dependent upon the remedy selected for the 
LAOCB. Estimated costs associated with Alternative P-4 are summarized below. Costs for this alternative are provided in Appendix B.

\begin{tabular}{|c|c|}
\hline Capital Costs & $\$ 275,000$ \\
\hline O\&M Costs & $\$ \quad 5,00$ \\
\hline Total Costs & \\
\hline
\end{tabular}

\section{Alternative P-5: $\quad$ In-Situ High Pressure Wash and In-Situ S/S}

Under Alternative P-5, the pipelines would be washed using water under high pressure. The pipelines would then be filled with grout in-situ. The majority of residual contaminants within the pipelines would likely be washed out of the pipelines under a high pressure wash and into the LAOCB. However, removal of contamination on the internal surfaces of the pipelines by high pressure washing would be difficult given the length of the pipelines, and the degree of corrosion and rust on the internal surface of the pipelines that have likely bound radionuclides to the surface of the metal. Once in the LAOCB, the washwater could evaporate and the radionuclides would remain in the basin for potential future remediation. Grouting would immobilize remaining contaminants within each pipeline.

Washing the pipelines would risk the spread of contamination if cracks or stressed points in the pipes allowed contaminated water to escape. The risk of contaminated grout escaping also exists as discussed under Alternative P-4. Based on current knowledge of the pipeline areas (reviewed in Section 3.2.2.1), the pipelines are not a source of radionuclide contamination to surrounding soils and/or groundwater. Therefore, due to the additional risk to the environment caused by these activities, the added effectiveness provided by this alternative is uncertain. Additionally, washing the pipelines would cause the radioisotopes to disassociate from the interior of the pipeline and create a contaminated waste stream of larger volume. Future failure of pipeline integrity could also release radionuclides to surrounding soils. Therefore, protection of the environment cannot be guaranteed for the future. 
Implementation of this alternative would include washing and grouting the pipelines and capping the pipeline areas. High pressure washing would require very conventional equipment, but would exposure workers to the interior of the pipeline and could create a spray coming from the basin-end of the pipe or a backspray at the LAHS end of the pipe. Grouting would require in-situ S/S equipment that should be readily available, but could expose remedial workers to contaminants. However, contaminant exposure can be minimized and maintained below acceptable levels with the use of proper protective clothing, scheduled monitoring of area radiation and airborne particulate levels, and strict adherence to the project health and safety plan.

Costs associated with Alternative P-5 would include materials \& equipment required for washing, grouting, and capping the two pipelines. A review of remedy would be required every five years for 30 years. The remedy review costs $(\$ 280,000)$ would be included in that for the LAOCB OU, dependent upon the remedy selected for the LAOCB. Estimated costs associated with Alternative P-5 are summarized below. Costs for this alternative are provided in Appendix B.

\section{Capital Costs $\$ 400,000$ \\ O\&M Costs $\$ 5,000$ \\ Total Costs $\$ 405,000$}

Evaluation of Alternatives $P_{-4}$ and P-5

Both Alternatives are implementable. There would be some risk to remedial workers associated with implementation of either alternative, but the risk could be minimized through safety precautions. Under Alternative P-5, high pressure washing could remove the contaminants in the pipeline and discharge the contaminants to the LAOCB. However, removal of contamination on the internal surfaces of the pipelines by high pressure washing would be difficult given the length 
of the pipelines and the degree of corrosion and rust that has likely bound radionuclides to the internal surface of the metal on the internal surfaces of the pipelines.

Under both Alternatives P-4 and P-5, grouting would seal residual radioisotopes between the grout and the pipeline wall. Based on the shallow depth of the pipeline [within $0.91 \mathrm{~m}(3 \mathrm{ft})$ of the ground surface], its relatively high radioactivity level (approximately 300,000 dpm on internal surface), the age ( $>30$ years) and material or the pipe (cast iron which could degrade over time and release radioisotopes), future failure of pipeline integrity could result in the release of radionuclides to the environment. Neither alternative would be effective at preventing the migration of contaminants from the pipelines to the environment. Alternatives P- 4 and P-5 will not be retained for detailed analysis.

\subsubsection{Alternatives Under the General Response of Disposal}

Two alternatives are categorized under the general response of disposal: Alternative P-6, (in-situ S/S, excavation, and disposal in the LAOCB); and Alternative P-7 [in-situ S/S, excavation, and disposal at the Nevada Test Site (NTS)]. Following are descriptions, screening, and evaluation of Alternatives P-6 and P-7.

Alternative P-6: $\quad$ In-situ $S / S$, Excavation and Disposal in the $L A O C B$

Under Alternative P-6, the pipelines would be grouted, excavated, downsized by cutting, and disposed of in the LAOCB. The pipelines would be grouted, and excavated using conventional excavation equipment, cut into sections, and placed in the LAOCB. The LAOCB has the capacity to accommodate disposal of the pipelines and associated contaminated materials once the pipe has been downsized. Following the removal of all pipeline and associated contaminated materials, the pipeline area would be backfilled to grade and made available for future use. 
Removal of the pipelines without first grouting the inside could potentially release residual contaminants from inside the pipeline to the environment. In addition, such a release could also increase risk to remedial workers and increase the mobility and volume of contaminants during removal activities. In-situ S/S (grouting) of the pipeline, as presented in Alternative P-4, prior to excavation, removal, and final disposal inside the LAOCB would immobilize contaminants and reduce risk.

If the grouted pipelines are excavated and disposed of inside the LAOCB, the remaining void space in the basin would be grouted with the same mixture used for in-situ S/S. Grouting around the pipeline sections inside the basin would further encapsulate the outside of the pipelines, and create a monolith that would further reduce the mobility of the pipeline contaminants.

Since the LAOCB pipelines and any associated contamination would be removed from the pipeline area, Alternative P-6 would be protective of human health and the environment. Therefore, Alternative P-6 meets the remedial action objective of mitigating the release of primary and secondary COCs to unit groundwater and minimizing risk to human health and the environment.

Implementation of this alternative would include grouting, excavation, pipeline downsizing, and backfilling activities. The alternative would be implementable, although certain precautions regarding radioactive waste removal would be required. As presented under Alternative P-4, exposure to remedial workers would be a concern under this alternative. However, risk could be minimized to below acceptable levels with the use of proper protective clothing, scheduled monitoring of area radiation and airborne particulate levels, and strict adherence to the project health and safety plan.

Costs associated with Alternative P-6 would include grouting, excavating, pipe cutting, and backfilling equipment. Cost associated with in-situ S/S after disposal inside the LAOCB are 
presented under Alternative S-4 (In-Situ stabilize, Backfill and Cap the LAOCB). No maintenance costs would be associated with this alternative because contamination would be removed from the area. Estimated costs associated with Alternative P-6 are summarized below. Costs for this alternative are provided in Appendix B.

$$
\begin{array}{lr}
\text { Capital Costs } & \$ 990,000 \\
\text { O\&M Costs } & \$ \$ 0 \\
\text { Total Costs } & \$ 990,000
\end{array}
$$

Alternative P-7: $\quad$ In-situ S/S, Excavation and Disposal at the NTS

Under Alternative P-7, the pipelines would be grouted, excavated, downsized by cutting, and subsequently transported to the NTS for disposal. Pipelines would be grouted and excavated using conventional excavation equipment and then, once downsized, placed in roll-off boxes which would transported by truck to the NTS and subsequently disposed. Following the removal of all pipeline and associated contaminated materials, the pipeline area would be backfilled to grade and made available for future use.

Removal of the pipelines without first grouting the inside could potentially release residual contaminants from inside the pipeline to the environment. In addition, such a release could also increase risk to remedial workers and increase the mobility and volume of contaminants during removal activities. In-situ S/S (grouting) of the pipeline, as presented in Alternative P-4, prior to excavation, removal, and final disposal inside the LAOCB would immobilize contaminants and reduce risk.

Alternative P-7 would be protective of human health and the environment since the LAOCB pipelines and associated contamination would be removed from the pipeline area. Furthermore, risk to remedial workers, associated with the excavation and downsizing of the pipelines, would be minimized by grouting the pipeline prior to these activities. Therefore, Alternative P-7 meets 
the remedial action objective of mitigating the leaching of primary and secondary COCs to unit groundwater and reducing risk to human health and the environment.

Implementation of this alternative would include grouting, excavation, pipeline downsizing, and backfilling activities. Specialized waste handling equipment may be required to reduce contaminant mobility of airborne particulate emissions. However, the required equipment should be available. Exposure to remedial workers during excavation activities would be minimized, but still of concern under this alternative. However, risk could be maintained below acceptable levels with the use of proper protective clothing, scheduled monitoring of area radiation and airborne particulate levels, and strict adherence to the project health and safety plan. There may also be public concern prompted due to the long distance transport to Nevada.

Costs associated with Alternative P-7 would include excavation and backfilling equipment, and transportation and disposal fees. No maintenance costs would be associated with this alternative because contamination would be removed from the area. Estimated costs associated with Alternative P-7 are summarized below. Costs for this alternative are provided in Appendix B.

\author{
Capital Costs $\$ 4,630,000$ \\ O\&M Costs $\$$ \\ Total Costs $\$ 4,630,000$
}

Evaluation of Alternatives $P-6$ and $P-7$

Both alternatives include the grouting, excavation, and disposal of the LAOCB pipelines. However, Alternative P-7 would require long distance travel for disposal at the NTS. Both alternatives would meet remedial action objectives and would leave the pipeline areas free of contamination and available for future use. Both alternatives are also implementable, but would involve a risk to remedial workers due to pipeline handling. Alternative P-7 may be met with 
public concern due to the long distance transport of the pipeline and is more costly than Alternative P-6. Both alternatives are retained for detailed analysis.

\subsubsection{Summary of LAOCB Pipeline Alternatives Screening}

Alternatives P-1, P-2, P-4, and P-5 cannot guarantee the prevention of future human exposure or future prevention of radionuclide migration into the environment. None of these alternatives, except for Alternative P-1, is retained for detailed analysis. Alternative P-1 is retained in accordance with NCP requirements as a baseline for comparison of other alternatives. In addition to P-1, Alternatives P-3, P-6, and P-7 were also retained for detailed analysis. Alternative P-3 provides additional measures to further insure that contaminants within the pipelines do not migrate. Alternatives P-6 and P-7 both involve disposal which would remove the pipelines and associated contamination from the area and the land would be available for future use.

Alternatives P-6 and P-7 could involve a risk to remedial workers due to the excavation and grouting activities involved. Alternative P-7 would likely prompt public concern due to the long distance transport to the NTS facility. However, all alternatives are implementable and risks should be manageable.

Costs for implementation of the alternatives include materials and equipment. Five year remedy reviews would be required with Alternative P-1, and P-3 since contamination would remain in place that would prohibit unlimited use of the land; however, the review of remedy costs $(\$ 280,000)$ would be included in that of the LAOCB OU, dependent upon the remedy selected for the LAOCB. No alternatives were rejected on the basis of cost. Therefore, the remaining alternatives were retained for detailed analysis. 
The retained alternatives were renumbered in sequential order for further discussion in Section 4.

The retained alternatives are identified as follows in the remainder of this document:

Alternative P-1: $\quad$ No Action

Alternative P-2: Capping

Alternative P-3: In-situ S/S, Excavation and Disposal in the LAOCB

Alternative P-4: $\quad$ Excavation and Disposal at the NTS 


\section{CHAPTER 3}

FIGURES 
THIS PAGE INTENTIONALLY LEFT BLANK 


\section{CONCENTRATION (ACTIVITY) OF CESIUM-137 AND COBALT-60 OVER TIME}
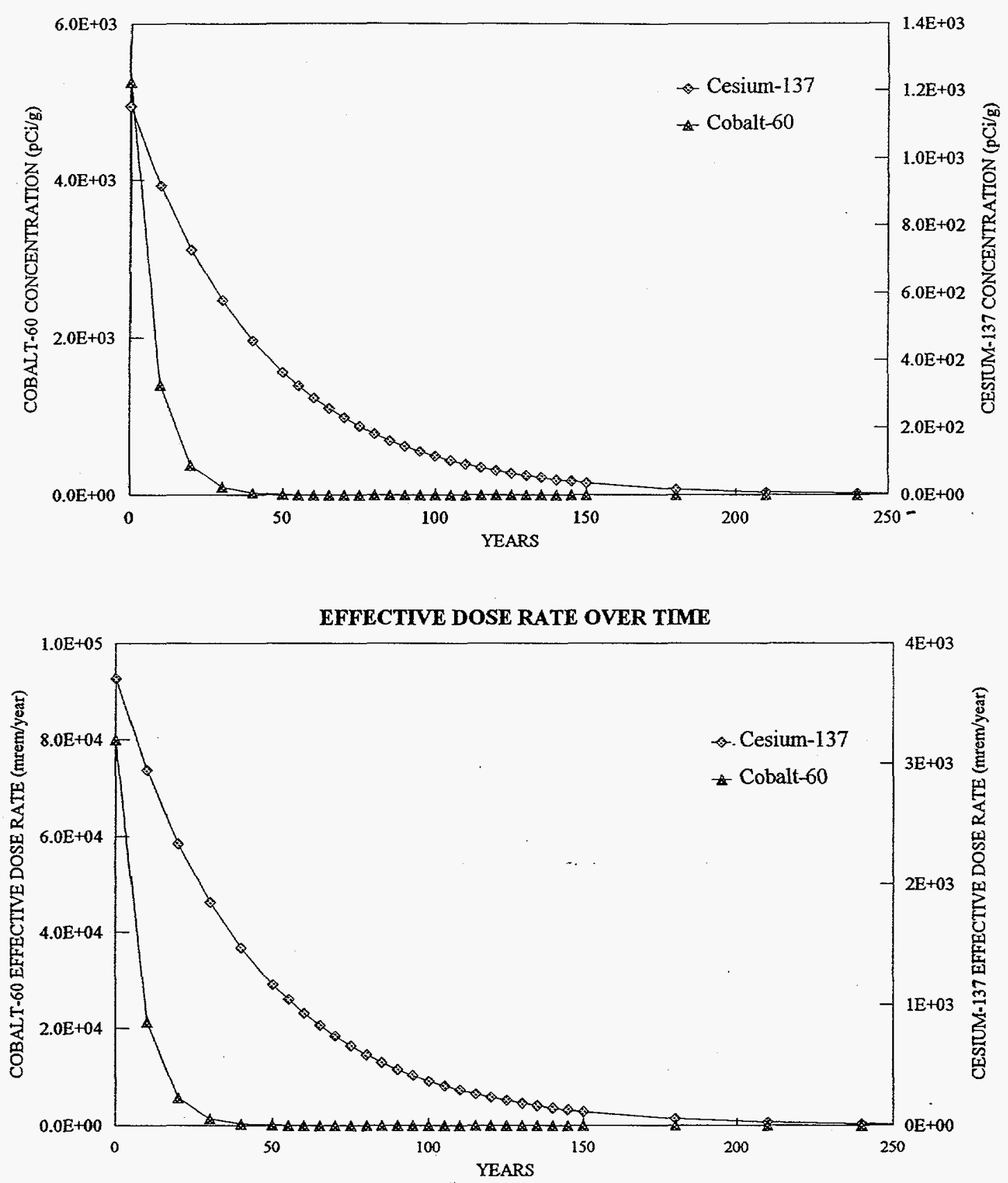

Notes:

Cobalt-60 (1/2 Life $=5.26$ years $)$ Effective Dose Rate will be reduced from $191,018 \mathrm{mrem} /$ year to $100 \mathrm{mrem} /$ year in approximately 57 years and $25 \mathrm{mrem} /$ year in approximately 67 years.

Cesium-137 (1/2 Life $=30$ years $)$ Effective Dose Rate will be reduced from $4,320 \mathrm{mrem} /$ year to $100 \mathrm{mrem} / \mathrm{year}$ in approximately 112 years and $25 \mathrm{mrem} /$ year in approximately 212 years.

Figure 3-1a Reduction in Cesium-137 and Cobalt-60 Concentration and Corresponding Annual Effective Dose Rate through Radioactive Decay 
THIS PAGE INTENTIONALLY LEFT BLANK 
CESIUM-137

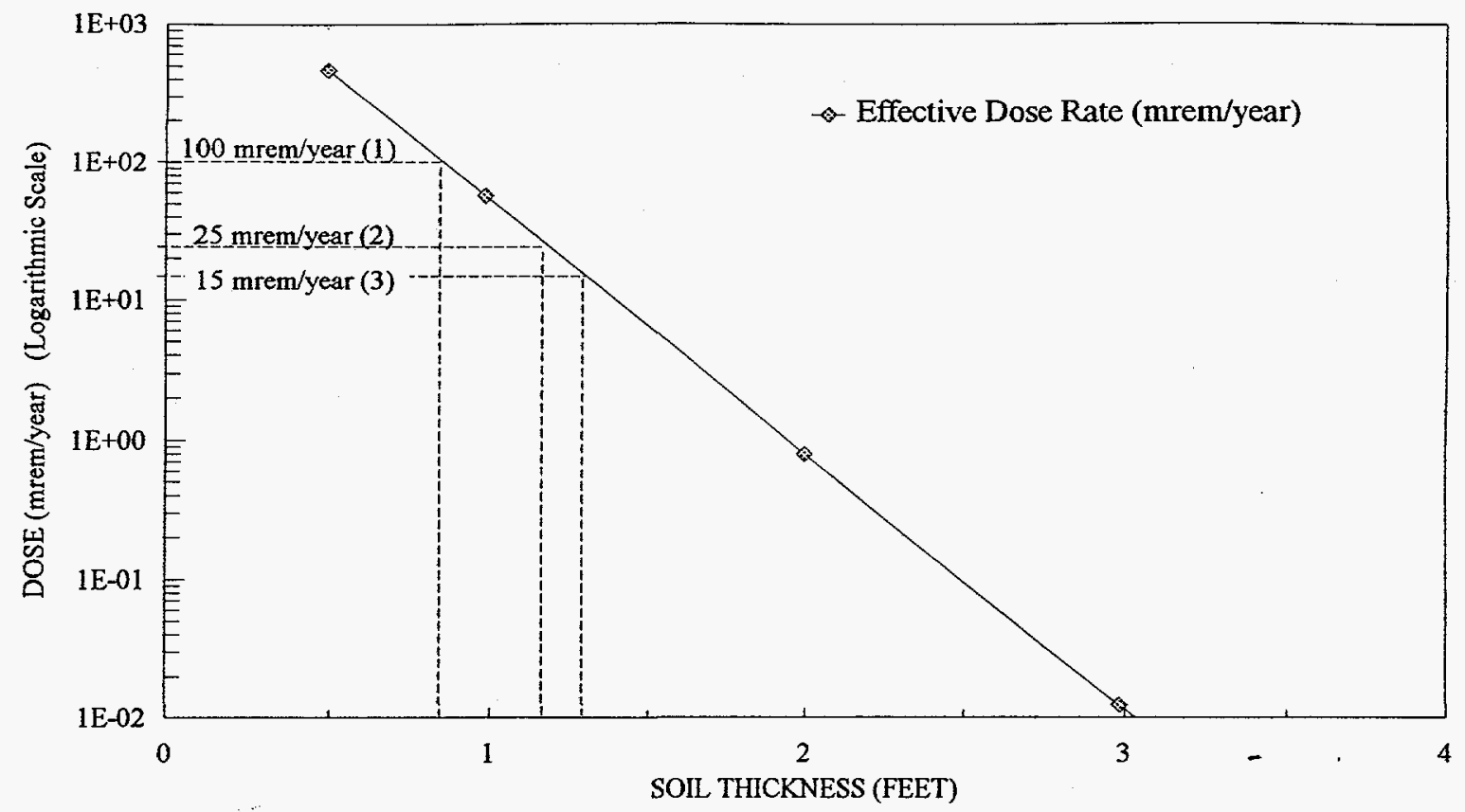

COBALT-60

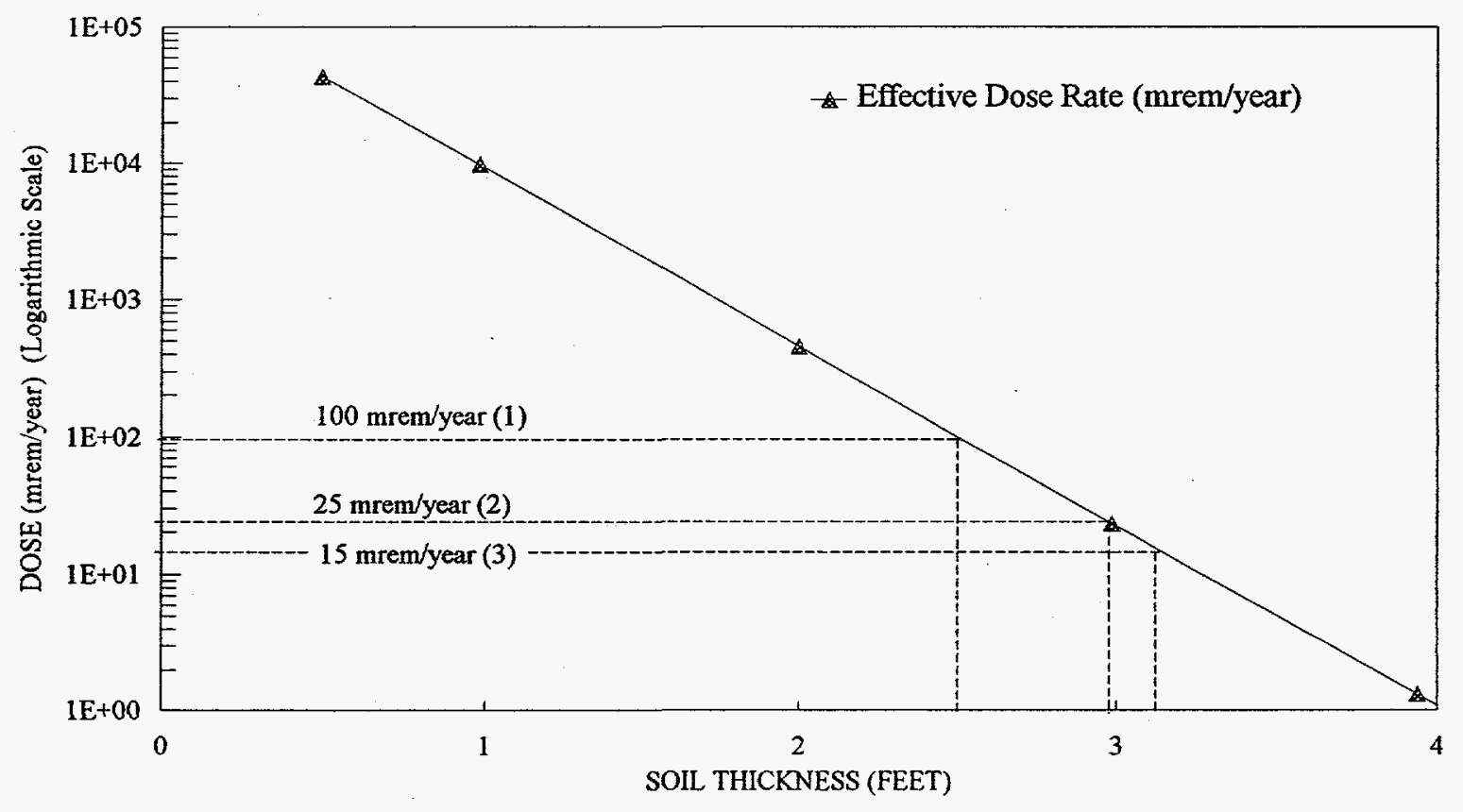

Notes:

1. Allowable dose to public (DOE order 5400.5)

2. Allowable dose to public ( 40 CFR 192)

3. Proposed regulation

Figure 3-1b Annual Effective Dose Rate Reduction through Capping 
CAP Area: $\quad 2,048 \mathrm{~m}^{2} \quad(22,040 \mathrm{ft} 2 ; 0.51$ acres $)$

CAP Perimeter: $\quad 187 \mathrm{~m} \quad(612 \mathrm{ft})$

Drainage Fabric Area: $2,341 \mathrm{~m} 2 \quad(25,200 \mathrm{ft} 2 ; 0.58$ acres $)$

(Extra $5 \mathrm{ft}$ required per side to anchor)

Liner Area: $\quad 2,221 \mathrm{~m}^{2} \quad(23,912 \mathrm{ft} 2 ; 0.55$ acres $)$

(Extra $3 \mathrm{ft}$ required per side to anchor)

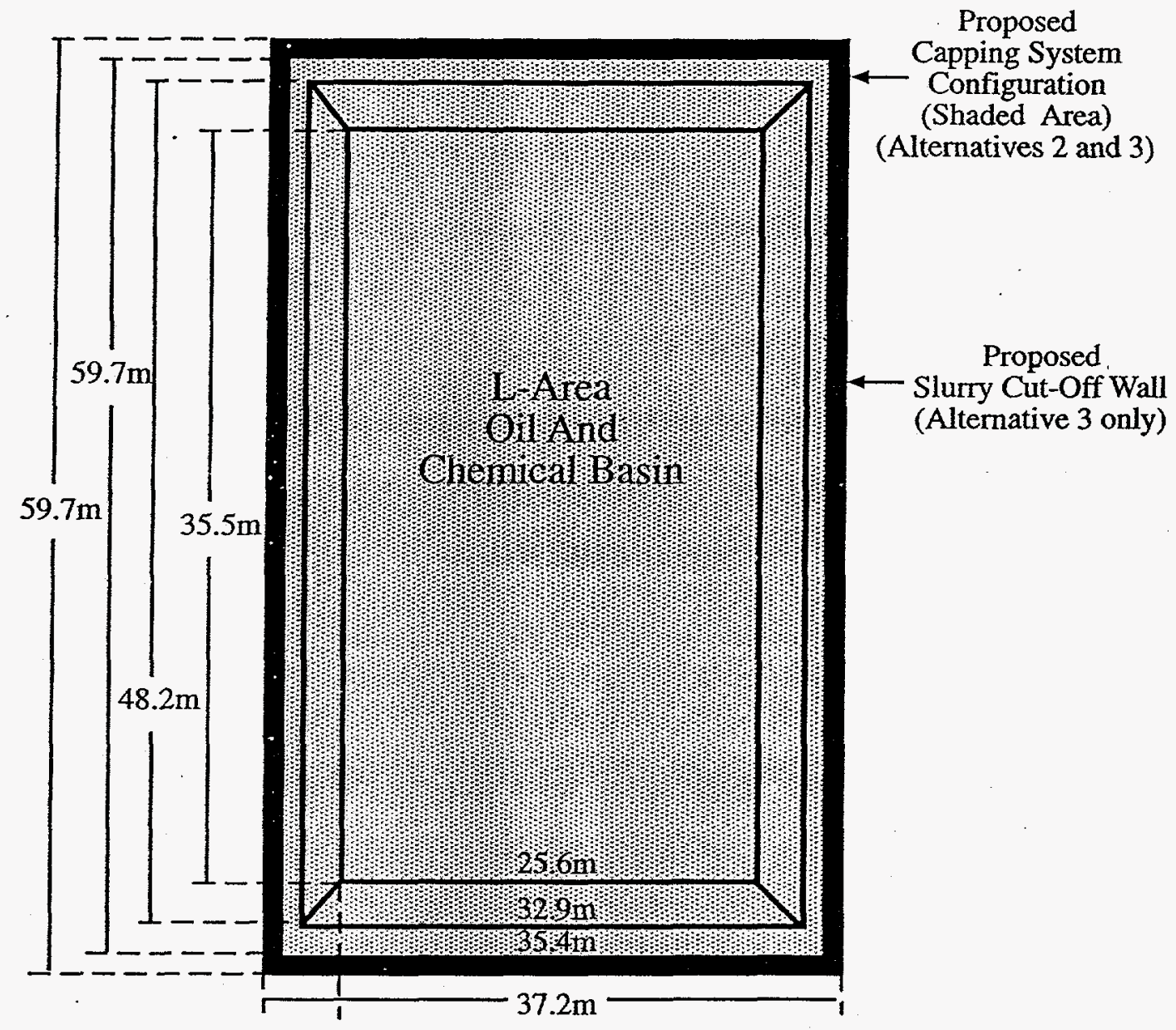

Site

North

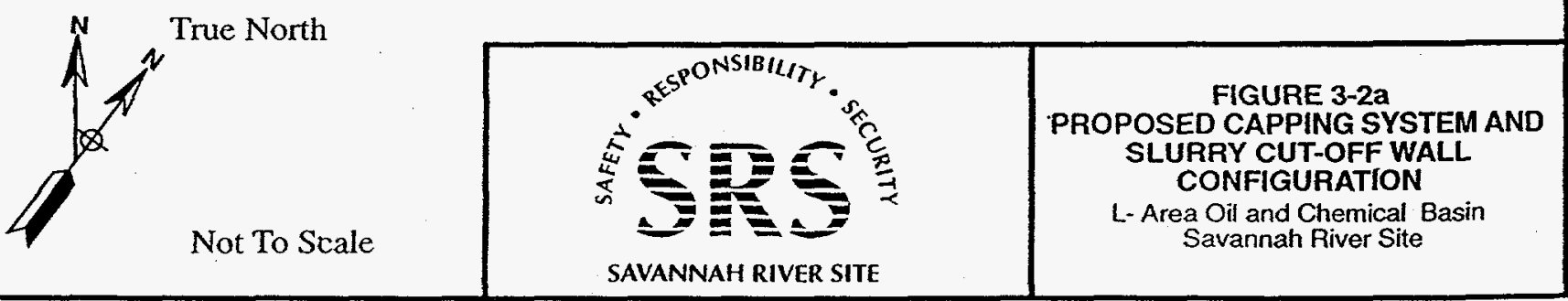


PHASE I FOCUSED CMS/FS REPORT

L-AREA OIL AND CHEMICAL BASIN
WSRC-RP-96-106, REV. 1.1 FEBRUARY 1997

THIS PAGE INTENTIONALLY LEFT BLANK 


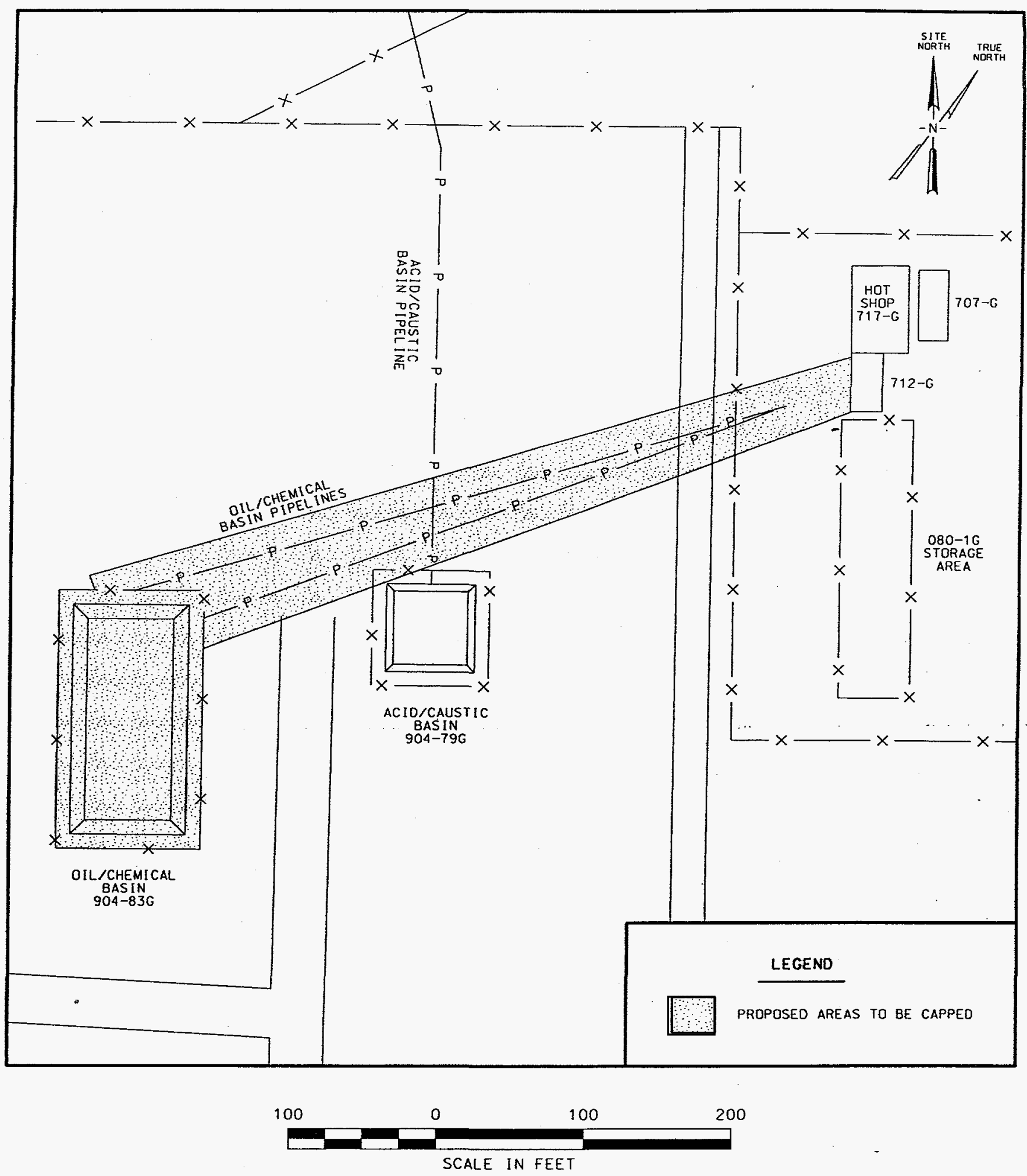

Figure 3-2b Proposed Capping System for the LAOCB and Pipelines 
THIS PAGE INTENTIONALLY LEFT BLANK 


\section{CHAPTER 3}

TABLES 
TABLE 3-1

SUMMARY OF REMEDIAL ALTERNATIVES SCREENING

L-AREA OIL AND CHEMICAL BASIN SOIL

SAVANNAH RIVER SITE

\begin{tabular}{|c|c|c|c|c|}
\hline General Response/Alternative & Effectiveness & Implementability & Budgetary Cost $^{1}$ & Status \\
\hline No Action/Alternative S-1 & $\begin{array}{l}\text { Not effective in reducing } \\
\text { risk }\end{array}$ & Immediate & $\$ 280,000$ & Retained \\
\hline \multicolumn{5}{|l|}{ Containment/ } \\
\hline Alternative S-2 - Capping & $\begin{array}{l}\text { Attenuates beta/gamma } \\
\text { radiation and prevents } \\
\text { migration }\end{array}$ & $\begin{array}{l}\text { Requires no waste handling; } \\
\text { requires long-term } \\
\text { maintenance }\end{array}$ & $\$ 1,430,000$ & Retained \\
\hline Alternative S-3 - Slurry Cut-Off Wall \& Capping & $\begin{array}{l}\text { Attenuates beta/gamma } \\
\text { radiation and prevents } \\
\text { migration }\end{array}$ & $\begin{array}{l}\text { Requires no waste handling; } \\
\text { difficult to verify } \\
\text { effectiveness; requires long- } \\
\text { term maintenance }\end{array}$ & $\$ 3,430,000$ & Retained \\
\hline \multicolumn{5}{|l|}{ h-Situ Treatment } \\
\hline Alternative S-4 - In-Situ Stabilization \& Capping & $\begin{array}{l}\text { Attenuates beta/gamma } \\
\text { radiation and minimizes } \\
\text { leachability }\end{array}$ & $\begin{array}{c}\text { Requires little waste } \\
\text { handling; difficult to verify } \\
\text { effectiveness; requires long- } \\
\text { term maintenance }\end{array}$ & $\$ 3,580,000$ & Retained \\
\hline Alternative S-5 - In-Situ Vitrification \& Capping & $\begin{array}{c}\text { Attenuates beta/gamma } \\
\text { radiation and minimizes } \\
\text { leachability }\end{array}$ & $\begin{array}{c}\text { Requires little waste } \\
\text { handling; cap requires long- } \\
\text { term maintenance }\end{array}$ & $\$ 7,260,000$ & Rejected \\
\hline
\end{tabular}


TABLE 3-1

SUMMARY OF REMEDIAL ALTERNATIVES SCREENING

L-AREA OIL AND CHEMICAL BASIN SOIL

SAVANNAH RIVER SITE

\begin{tabular}{|c|c|c|c|c|}
\hline General Response/Alternative & Effectiveness & Implementability & Budgetary Cost ${ }^{1}$ & Status \\
\hline Alternative S-6 - Ex-Situ Stabilization \& Capping & $\begin{array}{c}\text { Attenuates beta/gamma } \\
\text { radiation and minimizes } \\
\text {. leachability }\end{array}$ & $\begin{array}{l}\text { Requires pre-excavation } \\
\text { treatment for waste handling } \\
\text { purposes; extensive waste } \\
\text { handling and long-term cap } \\
\text { maintenance }\end{array}$ & $\$ 4,370,000$ & Retained \\
\hline \multicolumn{5}{|c|}{ Off Unit Disposal } \\
\hline $\begin{array}{l}\text { Alternative S-8 - Excavation \& Disposal at the Soils } \\
\text { Consolidation Facility }\end{array}$ & $\begin{array}{l}\text { Removes source of } \\
\text { contamination; little } \\
\text { residual risk to human } \\
\text { health or the environment }\end{array}$ & $\begin{array}{l}\text { Requires pre- \& post- } \\
\text { excavation treatment for } \\
\text { waste handling/packaging } \\
\text { purposes; extensive waste } \\
\text { handling and transport; } \\
\text { increased worker exposure; } \\
\text { significant risk to public }\end{array}$ & Unknown ${ }^{2}$ & $\begin{array}{l}\text { Temporaril } \\
\text { Rejected }\end{array}$ \\
\hline $\begin{array}{l}\text { Alternative S-9 - Excavation \& Disposal at the } \\
\text { Nevada Test Site Facility }\end{array}$ & $\begin{array}{l}\text { Removes source of } \\
\text { contamination; little } \\
\text { residual risk to human } \\
\text { health or the environment }\end{array}$ & $\begin{array}{l}\text { Requires pre- \& post- } \\
\text { excavation treatment for } \\
\text { waste handling/packaging } \\
\text { purposes; extensive waste } \\
\text { handling and transport; } \\
\text { increased Worker exposure; } \\
\text { significant risk to public }\end{array}$ & $\$ 9,100,000$ & Retained \\
\hline
\end{tabular}


TABLE 3-1

SUMMARY OF REMEDIAL ALTERNATIVES SCREENING

L-AREA OIL AND CHEMICAL BASIN SOIL

SAVANNAH RIVER SITE

\begin{tabular}{|c|c|c|c|c|}
\hline General Response/Alternative & Effectiveness & Implementability & Budgetary Cost ${ }^{\prime}$ & Status \\
\hline $\begin{array}{l}\text { Alternative S-10 - Excavation \& Disposal at the } \\
\text { Envirocare Facility }\end{array}$ & $\begin{array}{l}\text { Removes source of } \\
\text { contamination; little } \\
\text { residual risk to human } \\
\text { health or the environment }\end{array}$ & $\begin{array}{l}\text { Requires pre- \& post- } \\
\text { excavation treatment for } \\
\text { waste handling/packaging } \\
\text { purposes; extensive waste } \\
\text { handling and transport; } \\
\text { increased worker exposure; } \\
\text { significant risk to public }\end{array}$ & $\$ 13,040,000$ & 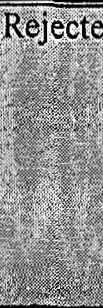 \\
\hline
\end{tabular}

'Costs are developed for comparison purposes only and are not intended to forecast actual expenditures.

${ }^{2}$ Costs are not known since the Soils Consolidation Facility has not been sited, designed, or constructed.

$\mathrm{S} / \mathrm{S}=$ Stabilization/Solidification

ğ 
TABLE 3-2

SUMMARY OF REMEDIAL ALTERNATIVES SCREENING

L-AREA OIL AND CHEMICAL BASIN PIPELINES

SAVANNAH RIVER SITE

\begin{tabular}{|c|c|c|c|c|}
\hline General Response/Alternative & Effectiveness & Implementability & Budgetary Cost ${ }^{1}$ & Status \\
\hline No Action/Alternative P-1 & $\begin{array}{l}\text { Effective with current controls; } \\
\text { radionuclide migration to } \\
\text { groundwater has not occurred }\end{array}$ & Immediate & See Note 2 below & Retained \\
\hline $\begin{array}{l}\text { Containment/Alternative P-3 - } \\
\text { Capping }\end{array}$ & Prevents potential migration & $\begin{array}{l}\text { Requires no waste handling; requires } \\
\text { long-term maintenance }\end{array}$ & $\$ 730,000$ & Retained \\
\hline Alternative P-4 - In-Situ S/S & $\begin{array}{l}\text { Not effective; protection from a } \\
\text { future release can not be guaranteed }\end{array}$ & $\begin{array}{l}\text { Requires little waste handling; difficult to } \\
\text { verify effectiveness; }\end{array}$ & $\$ 280,000$ & \\
\hline $\begin{array}{l}\text { Alternative P-5 - In-Situ High } \\
\text { Pressure Wash and S/S }\end{array}$ & $\begin{array}{l}\text { Not effective; protection from a } \\
\text { future release can not be guaranteed }\end{array}$ & $\begin{array}{l}\text { Requires little waste handling; difficult to } \\
\text { verify effectiveness; }\end{array}$ & $\$ 405,000$ & \\
\hline \multicolumn{5}{|c|}{ Bisposal? } \\
\hline $\begin{array}{l}\text { Alternative P-7 - Excavation \& } \\
\text { Disposal at the Nevada Test Site } \\
\text { Facility }\end{array}$ & $\begin{array}{l}\text { Removes source of contamination; } \\
\text { little residual risk to human health or } \\
\text { the environment }\end{array}$ & $\begin{array}{l}\text { Requires extensive waste handling and } \\
\text { transport; increased worker exposure; } \\
\text { risk to public from transportation } \\
\text { accidents }\end{array}$ & $\$ 4,630,000$ & Retained \\
\hline
\end{tabular}

1. Costs are developed for comparison purposes only and are not intended to forecast actual expenditures.

2. Cost for remedy review would be included in those for the LAOCB OU and dependent on the remedy selected for the LAOCB.

$\mathrm{S} / \mathrm{S}=$ Stabilization/Solidification 


\subsection{DETAILED ANALYSIS OF ALTERNATIVES}

Six potential remedial alternatives were formulated in Section 3.0 that address LAOCB contaminated soil. Four potential remedial alternatives were also formulated in Section 3.0 that address the LAOCB pipelines. The NCP requires that potential remedial alternatives undergo detailed analysis using relevant evaluation criteria that will be used by decision makers in selecting a final remedy. The results of the detailed analysis are then arrayed to compare alternatives and identify key trade-offs among alternatives. Figure 4-1 provides a schematic of the components of the detailed analysis of alternatives.

CERCLA and RCRA evaluation criteria are described in Section 4.1. Potential alternatives that address LAOCB soil are individually evaluated in detail against CERCLA and RCRA requirements in Section 4.2. Results of this assessment for all LAOCB soil alternatives are arrayed in Table 4-1 and are subjected to comparative analysis in Section 4.2.7. Potential alternatives that address the LAOCB pipelines are then evaluated in detail in Section 4.3. Results of this assessment for all pipeline alternatives are arrayed in Table 4-2 and are subjected to comparative analysis in Section 4.3.8.

\subsection{Introduction}

Detailed analyses of alternatives is required by the NCP (40 CFR 300.430(e)(9)). In this section, the alternatives formulated in Section 3.3 are evaluated in detail against CERCLA requirements. Following is a discussion of the evaluation criteria used to perform the detailed analyses of alternatives.

The statutory requirements that guide the evaluation of remedial alternatives in a CERCLA FS are that a remedial action must: 
- be protective of human health and the environment

- $\quad$ attain ARARs or define criteria for invoking a waiver

- be cost effective

- use permanent solutions to the maximum extent

EPA has established nine evaluation criteria to address these statutory requirements under CERCLA. The criteria fall into the categories of threshold criteria, primary balancing criteria, and modifying criteria. Section 4.2 presents an evaluation of each potential remedial alternative based on threshold and primary balancing criteria. Modifying criteria (State or support agency acceptance and community acceptance) will be evaluated after the public comment period on the CMS/FS and the Statement of Basis and Proposed Plan. Evaluation criteria categories and the nine evaluation criteria are listed and explained below. Figure 4-2 provides a summary of the components of the nine NCP criteria and Figure 4-3 provides the RCRA CMS criteria components. The CMS criteria are similar to the NCP criteria, with the exception of a CMS criterion which specifies the attainment of media protection standards. The media protection standards are not promulgated, and, therefore, will not be addressed in this CMS/FS.

\subsubsection{Threshold Criteria}

Threshold criteria are requirements that each alternative must achieve in order to be eligible for selection as a permanent remedy under CERCLA.

(1) Overall protection of human health and the environment - The overall protection of human health and the environment is evaluated for each alternative on the basis of how the alternatives reduce the risk of exposure to contaminants from potential exposure pathways through engineering or institutional controls. Each alternative is also examined as to whether it creates any unacceptable short-term risks to human health. 
(2) Compliance with ARARs - Location-specific and chemical-specific ARARs were identified and screened for relevance to the LAOCB waste unit in Section 2.3. Actionspecific ARARs pertaining to individual alternatives or remedial technologies are evaluated during detailed analyses.

\subsubsection{Primary Balancing Criteria}

Primary balancing criteria are factors that identify key tradeoffs among alternatives.

(3) Long-term effectiveness and permanence - Long-term effectiveness and permanence is evaluated for each alternative on the basis of the magnitude of residual risk and the adequacy and reliability of controls used to manage remaining waste after response objectives have been achieved. Alternatives that offer long-term effectiveness and permanence halt or otherwise mitigate any potential for off-site contaminant transport and minimize the need for future engineering controls.

(4) Short-term effectiveness - Evaluation of alternatives for short-term effectiveness takes into account protection of remedial workers, members of the community, and the environment during implementation of the remedial action and the time required to achieve cleanup goals. Schedule estimates are based on projected availability of materials and labor and may have to be updated at the time of remediation. Construction schedules are based on good weather, the ability to create and receive adequate and authorized access, and the availability of required utilities. All time estimates assume that the selected Remedial Design, including construction drawings, have been approved and all negotiations with contractors and regulators have been concluded.

(5) Reduction of mobility, toxicity, or volume through treatment - The statutory preference is to select a remedial action that employs treatment to reduce the toxicity, mobility, or 
volume of hazardous substances. The degree to which alternatives employ recycling or treatment is assessed, including how treatment is used to address the principal threats posed by the site.

(6) Implementability - Each alternative is evaluated with respect to the technical and administrative feasibility of implementing the alternatives as well as the availability of necessary equipment and services. This criterion includes such items as the ability to obtain services, capacities, equipment, and specialists necessary to construct components of the alternatives; the ability to operate the technologies and monitor their performance and effectiveness; and the ability to obtain necessary approvals from other agencies.

(7) Cost - Accuracy of present worth costs is $+50 /-30$ percent, per EPA guidance. Detailed cost estimates are derived from current information including vendor quotes, conventional cost estimating guides (e.g., Means Site Work Cost Data), and costs associated with similar projects: The cost estimates are included for comparison only and are not intended to forecast actual budgetary expenditures. The actual costs of the project would depend on labor and material costs, site conditions, competitive market conditions, final project scope, and implementation schedule at the time that the remedial activities are initiated. In estimating the present worth costs, a discount rate of five percent is usedand inflation is assumed to be zero percent. Present worth costs for review of the site remedy every five years are given for each alternative in which residuals would remain at the site. Present worth costs for these items are based on 30 years of operation, the maximum time allowed by EPA guidance. Cost estimates are presented in Appendix B.

\subsubsection{Primary Modifying Criteria}

Modifying criteria (State or support agency acceptance; community acceptance) will be considered during remedy selection. 
$(8,9)$ State or support agency acceptance and community acceptance - The preferred alternative should be acceptable to State and support agencies. Also, the concerns of the community should be considered in presenting alternatives that would be acceptable to the community. These two criteria would be evaluated based on comments on the CMS/FS Report and the Statement of Basis and Proposed Plan received during the public comment period and would be addressed in the final Statement of Basis and Proposed Plan prior to issuance of a RCRA permit modification and finalization of the ROD.

\subsection{Individual Analysis Of Soil Alternatives}

Remedial alternatives for the LAOCB contaminated soil are evaluated against the threshold and primary balancing criteria in the following sections.

\subsubsection{Alternative S-1: No Action}

Under the No Action alternative, no remedial efforts would be conducted to remove, treat, or otherwise lessen the toxicity, mobility, or affected volume of contaminated soil and all contaminated soil would remain in place. No treatment or additional institutional controls beyond that of existing SRS institutional controls would be performed under the No Action alternative.

\section{Overall Protection of Human Health and the Environment}

Alternative S-1 would be protective of human health as long as existing institutional controls are maintained. The No Action alternative would not eliminate or minimize potential routes for human exposure nor would it involve treatment to reduce the inherent risk associated with 
contaminated media. The existence of the clay layer (hardpan) under the Basin would prevent the migration of $\mathrm{COCs}$, thereby protecting groundwater and the environment.

The maximum concentrations of ${ }^{137} \mathrm{Cs}$ and ${ }^{60} \mathrm{Co}$ within the top $0.6 \mathrm{~m}(2 \mathrm{ft})$ of soil are $1,154 \mathrm{pCi} / \mathrm{g}$ and 5,242 pCi/g, respectively (Section 2.3.2.2). The effective external dose equivalents based on a one year exposure would be $2,707 \mathrm{mrem} /$ year and $79,977 \mathrm{mrem} /$ year, respectively. For protection of human health, 40 CFR 192 and DOE Order 5400.5 specifies an allowable dose equivalent to the public of $25 \mathrm{mrem} / \mathrm{year}$ and $100 \mathrm{mrem} / \mathrm{year}$, respectively (Section 2.3.2.1). Based on the half-life of ${ }^{137} \mathrm{Cs}$ (30 years; Table 1-3), the estimated time for radioactive decay to reduce the concentration of ${ }^{137} \mathrm{Cs}$ so as not to cause an effective dose equivalent greater than 25 mrem/year or $100 \mathrm{mrem} /$ year is approximately 166 and 106 years, respectively (Figure 3-1a). Based on the half-life of ${ }^{60} \mathrm{Co}$ (5.26 years; Table 1-3), the estimated time for radioactive decay to reduce the concentration of ${ }^{60} \mathrm{Co}$ so as not to cause an effective dose equivalent greater than 25 $\mathrm{mrem} /$ year or $100 \mathrm{mrem} /$ year is approximately 41 and 30 years, respectively (Table $3-1 \mathrm{a})$.

Long-term protectiveness of human health against ${ }^{137} \mathrm{Cs}$ and ${ }^{60} \mathrm{Co}$ would be achieved under the No Action alternative as long as existing institutional controls are maintained for at least 166 years. SRS institutional controls prohibit public access or entry to the LAOCB; therefore, the public is not exposed to the LAOCB contaminants. Although institutional controls are likely to be retained at SRS for the foreseeable future, the controls cannot be guaranteed for the length of time necessary for radioactive contaminants to decay to within regulatory limits. Therefore, whether the No Action alternative can achieve long term protectiveness of human health is questionable.

Any reduction in chemical concentrations in LAOCB media would occur as a result of natural mechanisms such as radioactive decay, sorption, dispersion, and dilution. The clay layer (hardpan) under the Basin serves as a retardant to COC migration from soil. Under this alternative, no additional efforts would be taken to contain LAOCB contamination or 
contaminated media. Based on data from the RFI/RI, identified COCs have not impacted groundwater at the Basin. Therefore, the clay layer appears to be an adequate barrier to COC migration. Future migration, however, could not be verified under the No Action alternative.

\section{Compliance with ARARs}

\section{Chemical-Specific ARARs}

The only nonradioactive chemical-specific ARARs for soil under Federal and South Carolina regulations are for PCBs. ARARs for PCBs are governed by TSCA (40 CFR Part 761). Based on the disposal history of the LAOCB, no PCBs are expected.

Of the radioactive constituents detected in soil, ${ }^{226} \mathrm{Ra},{ }^{228} \mathrm{Ra},{ }^{230} \mathrm{Th}$, and ${ }^{232} \mathrm{Th}$ are regulated through an ARAR and a TBC factor. These are:

- 40 CFR 192 Subparts A through E - Health and Environmental Protection Standards for Uranium and Thorium Mill Tailings

- DOE Order 5400.5 - Radiation Protection of the Public and the Environment

Sediment samples were not analyzed for alpha emitting $\mathrm{Ra}$ isotopes. Therefore, it is unknown whether the level of $\mathrm{Ra}$ in soil at the source area meets the ARAR and to-be-considered specified concentration of $\mathrm{Ra}$ in surface soil. No detectable activities of ${ }^{230} \mathrm{Th}$ or ${ }^{232} \mathrm{Th}$ were present in the Basin sediment. Soil in the area meet the ARAR and specified levels in DOE Order 5400.5.

The No Action alternative would not meet the 40 CFR 192 regulations or the to-be-considered DOE Order 5400.5 under the given assumptions should institutional controls cease to exist at SRS. Chemical-specific ARARs are discussed in detail in Section 2.3.2.1 of this document. 
Location-Specific ARARs

No location-specific ARARs are associated with the No Action alternative.

Action-Specific ARARs

No action-specific ARARs are associated with the No Action alternative.

\section{Long-Term Effectiveness and Permanence}

Long-term protectiveness of human health through mitigation of exposure to radiation resulting from the decay of ${ }^{60} \mathrm{Co}$ and ${ }^{137} \mathrm{Cs}$ would be achieved under the No Action alternative as long as existing institutional controls are maintained for at least 166 years. Although institutional controls are likely to be retained at SRS for the foreseeable future, the controls cannot be guaranteed for 166 years. Therefore, whether the No Action alternative can achieve long term protectiveness of human health is questionable. Because active institutional controls would be required for the foreseeable future, the No Action alternative would not be a permanent solution.

The clay layer (hardpan) beneath the Basin appears to adequately minimize the migration of COCs through the vadose zone. Under this alternative, no additional efforts would be taken to contain LAOCB contamination or contaminated media. Future migration of COCs would not be verifiable under the No Action alternative.

Reduction of Toxicity, Mobility, or Volume

The No Action alternative would not involve any form of active treatment that would reduce contaminant or contaminated media toxicity, mobility, or volume. 


\section{$\underline{\text { Short-Term Effectiveness }}$}

The No Action alternative would satisfy short-term protection of human health through existing institutional controls at SRS. Access to SRS is controlled at primary roads by continuously manned barricades. Other roads entering the site are closed to traffic by gates or barriers. Except along the Savannah River, the entire SRS facility is surrounded by an exclusion fence and is posted against trespassing under Federal and State statutes. The LAOCB is surrounded by a six foot high exclusion fence and posted as a Control Area (CA). Therefore, access directly to the Basin is further controlled beyond that of site security. Based on current conditions and existing institutional controls, exposure to LAOCB soil near the Basin should be minimal. In addition, Alternative S-1 would not involve any construction activities which would endanger public communities, remedial workers, or adversely impact the environment.

\section{Implementability}

The No Action alternative would require no construction, specialized equipment, or technical specialists and could therefore be implemented immediately. Alternative S-1 would not interfere with any possible future remedial actions at the LAOCB waste unit. On-going inspection of the CA fence would be conducted by SRS Radiological Control and Health Physics Department (HP) personnel. Access to the LAOCB and surrounding areas would be controlled through the designated custodian. If implemented, No Action would likely prompt public concern for protection of human health and the environment.

Alternative S-1 would not preclude any future remedial actions, if warranted. For instance, should the SRS Soil Consolidation Facility become operational, disposal of LAOCB wastes at the facility could be re-evaluated. 


\section{$\underline{\text { Cost }}$}

The only costs associated with the No Action alternative would be a review of remedy every five years for 30 years, as required by the NCP. The remedy review would include an assessment update for the LAOCB soil. Five year remedy reviews are required for remedial actions that result in hazardous substances, pollutants, or contaminants remaining at the waste unit above levels that allow for unlimited use and unrestricted exposure. No long-term operation and maintenance costs would be associated with the No Action alternative. Estimated costs associated with the No Action alternative do not include costs for existing institutional controls (e.g., security, groundwater monitoring). Costs are provided for comparison purposes only and are not intended to be used for forecasting actual budgetary expenditures. Estimated costs associated with the No Action alternative are estimated to be $\$ 280,000$. Itemized costs for the No Action alternative are provided in Appendix B.

\subsubsection{Alternative $S$-2: Capping}

Alternative S-2 consists of construction of a cap over the LAOCB. Although capping does not reduce the total mass of COCs and cannot achieve RGOs, capping is a performance based engineering approach that meets remedial action objectives by mitigating surface exposure to contaminants and reducing the leaching of contaminants to unit groundwater. As shown in Figure $3-1 \mathrm{~b}$, only $0.9 \mathrm{~m}(3 \mathrm{ft})$ of soil cover is required to reduce the annual effective dose associated with continuous exposure to the ${ }^{137} \mathrm{Cs}$ and ${ }^{60} \mathrm{Co}$ in the Basin by over 99 percent and within regulatory and DOE limits.

Initially, the waste unit would be prepared by clearing the vegetation, fencing, and other physical obstructions immediately surrounding the LAOCB. Prior to backfilling the Basin, the portion of the abandoned pipeline that is located beneath the proposed cap would be filled with grout, and contaminated media located on the side walls of the LOCB and in the staging area on the north 
end of the LAOCB would be placed in the bottom of the basin. The Basin would then be backfilled and compacted to grade. The approximate volume of backfill required is estimated to be $5,300 \mathrm{~m}^{3}\left(190,000 \mathrm{ft}^{3} ; 7,037 \mathrm{yd}^{3}\right)$. After sufficient compaction, an engineered cap would then be constructed over the LAOCB. The cap would cover an area of approximately 0.21 hectares ( 0.51 acres). A conceptual layout of the cap is illustrated in Figures 3-2a and 3-2b. For cost estimating purposes, a modified RCRA cap is assumed for this alternative since it would leave untreated contaminants in place. Cap design would be evaluated and selected during detailed design.

Existing SRS institutional controls would remain in effect and the capping system would be maintained for a minimum of 30 years.

\section{Overall Protection of Human Health and the Environment}

With proper design, construction, and maintenance, Alternative S-2 would be protective of human health and the environment. The cap would function as a physical barrier to prevent direct human exposure to soil-borne contamination, including ${ }^{137} \mathrm{Cs}$ and ${ }^{60} \mathrm{Co}$. The cap would also minimize infiltration and subsequent leaching of contamination from unsaturated soil to the groundwater. In addition, backfilling of the Basin with approximately $3.5 \mathrm{~m}(11.5 \mathrm{ft})$ of soil would effectively shield a hypothetical person standing upon the cap from alpha, beta, and

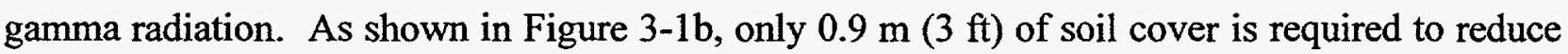
the annual effective dose associated with continuous exposure to the Basin by over 99 percent. Therefore, Alternative S-2 meets the remedial action objective by mitigating surface exposure to contaminants and reducing the leaching of tritium and primary and secondary $\mathrm{COCs}$ to the unit groundwater. 


\section{Compliance with ARARs}

\section{Chemical-Specific ARARs}

The discussion of the TSCA and UMTRCA ARARs identified under the No Action alternative (Section 4.2.1) applies to Alternative S-2. In regards to the public allowable effective dose equivalent specified in DOE Order 5400.5, capping of the LAOCB would reduce the effective dose equivalent by over 99 percent at the unit (Figure 3-1b). Implementation of Alternative S-2 would reduce the annual effective dose rate at the unit to below the allowable 25 mrem/year. Therefore, Alternative S-2 would achieve chemical-specific ARARs.

Location-Specific ARARs

No location-specific ARARs are associated with Alternative S-2.

Action-Specific ARARs

The capping system for Alternative S-2 would consist of a multi-layered RCRA cap. The particular design of the capping system would be selected during Remedial Design. A multi: layered RCRA cap must meet the performance standards of RCRA (40 CFR 264.310) as follows:

- $\quad$ provide long-term minimization of migration of liquids

- function with minimum maintenance

- $\quad$ promote drainage and minimize erosion or abrasion of the cover

- accommodate settling and subsidence to maintain cover integrity

- have a permeability less than that of natural subsurface soil

Numerous capping system designs would meet the RCRA performance standards; therefore, no difficulties can be perceived for designing a capping system under Alternative S-2. 
Section 112 of the Clean Air Act establishes the National Emissions Standards for Hazardous Air Pollutants (NESHAPs). Subpart H of 40 CFR 61 applies to facilities owned or operated by DOE. The requirements of Subpart $\mathrm{H}$ would be applicable to airborne emissions of radionuclides from the cleanup of the LAOCB unit. Air modeling would be required to ensure that no member of the public would receive an effective dose equivalent of $10 \mathrm{mrem} / \mathrm{year}$ (at the SRS boundary) from all SRS activities including LAOCB corrective measures/remedial activities. Should air modeling indicate that the effective dose equivalent solely from the cleanup of the LAOCB unit would be below $0.1 \mathrm{mrem} / \mathrm{year}$, only State notification would be required. Any dose equivalent above the specified limit would require a NESHAPs permit modification.

Workers performing the remediation at the $\mathrm{LAOCB}$ would be required to adhere to a project health and safety plan specified under the Occupational Safety and Health Association (OSHA; 29 CFR 1910).

\section{Long-Term Effectiveness and Permanence}

Under Alternative S-2, the magnitude of residual risks would be significantly reduced over current conditions. The potential for direct or indirect exposure to contaminants would be minimal as long as the cap is properly designed, constructed, and maintained. Caps have proven effective at many waste sites and should achieve design performance standards; however, should the cap ever be allowed to deteriorate, it would not function as designed. Under this scenario, precipitation could infiltrate into contaminated soil resulting in contaminant migration which is a threat to groundwater. However, the clay layer (hardpan) beneath the Basin appears to provide adequate protection of the groundwater by preventing $\mathrm{COC}$ migration. 
Existing institutional controls and scheduled maintenance would adequately maintain continued effectiveness of a cap at the LAOCB. Maintenance would include minor repairs to the cap cover and repairs and upkeep of the fence and warning signs. Major repairs may be required on occasion to address subsidence or similar threats to cap integrity, but this should not affect cap performance as long as such problems are promptly addressed.

Five year remedy reviews would be required under Alternative S-2 since contamination would remain at the waste unit above levels that would not allow for unrestricted use and unrestricted exposure.

\section{$\underline{\text { Reduction of Toxicity, Mobility, or Volume }}$}

Although Alternative S-2 would not involve any form of treatment that would reduce the toxicity, mobility, or volume of contaminants or contaminated media, it would effectively reduce contaminant mobility by minimizing infiltration and potential contaminant leaching, thereby further reducing inherent risks associated with soil-borne contamination. The existing clay layer (hardpan) that underlies the basin floor provides additional protection from COC migration to unit groundwater.

\section{Short-Term Effectiveness}

In general, cap construction is a straight-forward process requiring minimal disturbance of contaminated media. Although short-term risks to remedial workers would exist, the risks should be manageable. Exposure to remedial workers can be minimized and maintained well below acceptable levels with the use of proper protective clothing, scheduled monitoring of area radiation and airborne particulate levels, and strict adherence to the project health and safety plan. Consequently, short-term risks to the health of remedial workers would be minimal. In 
addition, Alternative S-2 should pose no significant short-term risk to the community and minimal risk to the environment.

Alternative S-2 should take approximately four months to complete after mobilization to the field. Concerns regarding radiation exposure during construction may cause periodic delays for the completion time.

Implementability

Alternative S-2 would be readily implementable. Depending upon the cap design selected, Alternative S-2 would require readily available materials (e.g., topsoil, clay, gravel, geosynthetics) and the use of conventional earth moving equipment (e.g., backhoes, dump trucks, bulldozers, compactors). Numerous qualified contractors are available that can competitively bid on the design and construction of an engineered cap.

Routine monitoring would be conducted to verify remedy effectiveness. Periodic monitoring of groundwater quality and cap subsidence would be conducted as part of ongoing institutional controls.

Alternative S-2 would not require any special permitting and would not directly involve any offunit activities. Caps have been commonly used at other low-level radioactive and mixed waste sites and generally do not elicit public concerns.

Alternative S-2 would not preclude any future remedial actions, if warranted. For instance, disposal of LAOCB wastes at the SRS Soil Consolidation Facility could be re-evaluated should the facility become operational. 
$\underline{\text { Cost }}$

Costs associated with Alternative S-2 include the labor and materials needed to construct the cap and operate and maintain the remedy for 30 years. Estimated costs do not include cap design costs or costs for existing institutional controls (e.g., security, groundwater monitoring). Operation and maintenance would include mowing and minor repairs to the cap and fence. Included in the present worth costs is a review of remedy every five years for 30 years, as required by the NCP. Costs are provided for comparison purposes only and are not intended to forecast actual budgetary expenditures. Estimated costs associated with Alternative S-2 are summarized below.

$\begin{array}{ll}\text { Capital Costs } & \$ 1,000,000 \\ \text { O\&M Costs } & \$ 430,000 \\ \text { Total Costs } & \$ 1,430,000\end{array}$

Costs for Alternative S-2 are provided in Appendix B.

\subsubsection{Alternative S-3: Slurry Cut-Off Wall and Capping}

Alternative S-3 consists of the construction of slurry cut-off walls around the perimeter of the LAOCB and a cap over the LAOCB. Although Alternative S-3 does not reduce the total mass of COCs and cannot achieve RGOs, it is a performance based engineering approach that meets remedial action objectives by mitigating surface exposure to contaminants and reducing the leaching of contaminants to unit groundwater. As shown in Figure 3-1b, only $0.9 \mathrm{~m}(3 \mathrm{ft})$ of soil cover is required to reduce the annual effective dose associated with continuous exposure to the ${ }^{137} \mathrm{Cs}$ and ${ }^{60} \mathrm{Co}$ in the Basin by over 99 percent and within regulatory and DOE limits. For cost estimating purposes, a modified RCRA cap is assumed for this alternative since it would leave untreated contaminants in-place. Slurry wall materials and design would be selected following 
treatability studies. Cap design would be evaluated and selected during Corrective Measures/Remedial Design.

Existing SRS institutional controls would remain in effect and the slurry wall and capping system would be maintained for a minimum of 30 years.

\section{Overall Protection of Human Health and the Environment}

Alternative S-3 would be protective of human health and the environment. The cap would function as a physical barrier to prevent direct human exposure to soil-borne contamination and would minimize infiltration and potential leaching of contaminants from unsaturated soil to the unit groundwater. If properly constructed and maintained, caps can provide effective long-term protection of human health and the environment. Coupled with a cap, the slurry wall would provide further reduction in contaminant mobility. Therefore, Alternative $S-3$ meets the remedial action objective of mitigating surface exposure to contaminants and reducing the leaching of tritium, and primary and secondary COCs to unit groundwater.

\section{Compliance with ARARs}

Chemical-specific, location-specific, and action-specific ARARs identified for Alternative S-2 (Section 4.2.2) would be the same as those under Alternative S-3.

\section{Long-Term Effectiveness and Permanence}

Under Alternative S-3, the magnitude of residual risks would be significantly reduced over current conditions. The potential for direct or indirect exposure to contaminants would be minimal as long as the cap and slurry wall are properly designed, constructed, and maintained. Caps and slurry walls have proven effective at many waste sites and should achieve design 
performance standards; however, should the cap ever be allowed to fail (e.g., termination of operation and maintenance), the cap would eventually deteriorate allowing water to accumulate within the slurry wall.

Existing institutional controls and scheduled maintenance would adequately maintain continued effectiveness of a slurry wall and cap at the LAOCB. Maintenance would include minor repairs to the cap cover and slurry wall and repairs and upkeep of the fence and warning signs. Major repairs may be required on occasion to address subsidence or similar threats to cap or slurry wall integrity, but this should not affect performance as long as such problems are promptly addressed.

Five year remedy reviews would be required under Alternative S-3 since contamination would remain at the waste unit above levels that would not allow for unrestricted use and unrestricted exposure.

\section{Reduction of Toxicity, Mobility, or Volume}

Backfilling of the Basin with approximately $3.5 \mathrm{~m}$ (11.5 ft) of soil would effectively shield a hypothetical person standing upon the cap from alpha, beta, and gamma radiation. As shown in.

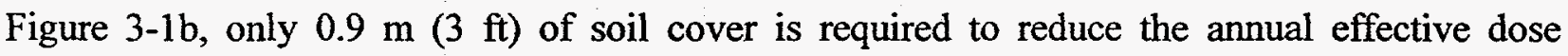
associated with continuous exposure to the Basin by over 99 percent. Although Alternative S-3 would not involve any form of treatment that would reduce the toxicity, mobility, or volume of contaminants or contaminated media, a slurry wall and cap would effectively reduce contaminant mobility by minimizing infiltration and contaminant leaching. In addition, the existing clay layer (hardpan) provides a natural barrier to contaminant migration. 


\section{Short-Term Effectiveness}

In general, slurry wall and cap construction are straight-forward processes requiring minimal disturbance of contaminated media. Although short-term risks to remedial workers would exist, the risks should be manageable. Exposure to remedial workers can be minimized and maintained below acceptable levels with the use of proper protective clothing, scheduled monitoring of area radiation and airborne particulate levels, and strict adherence to the project health and safety plan. Consequently, short-term risks to the health of remedial workers would be minimal. In addition, Alternative S-3 should pose no significant short-term risk to the community and minimal risk to the environment.

Alternative S-3 should take approximately six months to complete after mobilization to the field. Periodic delays could prolong the completion schedule due to potential inclement weather and safety concerns such as worker exposure to radiation.

\section{Implementability}

Alternative S-3 would be readily implementable. Depending upon the cap design selected, Alternative S-3 would require readily available materials (e.g., topsoil, clay, gravel; geosynthetics) and the use of conventional earth moving equipment (e.g., backhoes, dump trucks, bulldozers, compactors). Similarly, depending upon the slurry wall design selected, only readily available materials (e.g., soil, bentonite, cement) and excavation equipment would be required. Numerous qualified contractors are available that can competitively bid on the design and construction of a slurry wall and an engineered cap.

Routine monitoring would be conducted to verify remedy effectiveness. Periodic monitoring of groundwater quality and cap subsidence would be conducted as part of ongoing institutional controls. 
Alternative S-3 would not require any special permitting and would not directly involve any offunit activities. Caps and slurry walls have been commonly used at other low-level radioactive and mixed waste sites and generally do not elicit public concerns.

Alternative S-3 would not preclude any future remedial actions, if warranted. For instance, disposal of LAOCB wastes at the SRS Soil Consolidation Facility could be re-evaluated should the facility become operational.

\section{$\underline{\text { Cost }}$}

Costs associated with Alternative S-3 include the labor and materials needed to construct the cap and slurry wall and operate and maintain the remedy for 30 years. Estimated costs do not include cap or slurry wall design costs or costs for existing institutional controls (e.g., security, groundwater monitoring). Operation and maintenance would include mowing and minor repairs to the cap, slurry wall, and fence. Included in the present worth costs is a review of remedy every five years for 30 years, as required by the NCP. Costs are provided for comparison purposes only and are not intended to be used for budgetary purposes. Estimated costs associated with Alternative S-3 are summarized below.

$\begin{array}{ll}\text { Capital Costs } & \$ 3,000,000 \\ \text { O\&M Costs } & \$ 430,000 \\ \text { Total Costs } & \$ 3,430,000\end{array}$

Costs for Alternative S-3 are provided in Appendix B. 


\subsubsection{Alternative S-4: In-Situ Stabilization and Capping}

Alternative S-4 involves the in-situ stabilization of the identified LAOCB soil requiring remediation. The primary goal of in-situ $\mathrm{S} / \mathrm{S}$ is to minimize the mobility of primary and secondary COCs. Although in-situ S/S does not reduce the total mass of COCs and cannot in itself achieve RGOs, it is a performance based engineering approach that meets remedial action objectives by reducing the leaching of contaminants to unit groundwater. Results of the treatability study suggest in-situ $\mathrm{S} / \mathrm{S}$ will effectively reduce the mobility of primary and secondary COCs (WSRC, 1996c).

Upon completion of in-situ grouting, the LAOCB would be backfilled and compacted to grade, and the LAOCB would be capped as illustrated in Figures 3-2a and 3-2b and as described under Alternative S-2. For cost estimating purposes, a compacted clay cap is assumed since all known soil-borne contamination at the LAOCB waste unit would be treated under this alternative. The cap design would be selected during Corrective Measures/Remedial Design.

\section{Overall Protection of Human Health and the Environment}

Alternative S-4 would be protective of human health and the environment. The cap would function as a physical barrier to prevent direct human exposure to soil-borne contamination and would minimize infiltration and subsequent leaching of contamination from unsaturated soil to the groundwater. If properly constructed and maintained, caps can provide effective long-term protection of human health and the environment. Stabilization of LAOCB soil requiring remediation $(0.6 \mathrm{~m} ; 2 \mathrm{ft})$ would provide an added measure of long-term protection by immobilizing all known soil-borne contamination at the LAOCB waste unit. Therefore, Alternative S-4 meets the remedial action objectives of reducing surface exposure risk to human health and the environment and reducing the leaching of tritium and primary and secondary COCs to unit groundwater. 


\section{Compliance with ARARs}

Chemical-specific, location-specific, and action-specific ARARs identified for Alternative S-2 (Section 4.2.2) would be the same as that under Alternative S-4. However, Alternative S-4 would involve treatment of all known soil-borne contamination at the LAOCB unit. Therefore, the cap included under Alternative S- 4 would not be required to be a multi-layered cap design since all soil-borne contamination would be immobilized through treatment.

\section{Long-Term Effectiveness and Permanence}

Under Alternative S-4, the magnitude of residual risk remaining at the LAOCB would be low. With institutional controls and proper cap maintenance, an engineered cap would eliminate any route of direct exposure to contaminated soil. The cap would also minimize the potential for infiltration passing through the treated soil. Stabilization of all known soil-borne contamination at the LAOCB waste unit would add a significant margin of safety to the effectiveness of the cap. Grouting of soil would also prove advantageous for material handling should alternative remedial actions ever be required in the future.

Cap maintenance and occasional repair would be required for as long as radiation levels posed a risk to human health and the environment. Caps have proven effective at many waste sites and should achieve design performance standards. Existing institutional controls and scheduled maintenance would adequately maintain continued effectiveness of a cap at the LAOCB since SRS will be provided with institutional controls for the foreseeable future. Cap maintenance would include inspections for erosion, care for the vegetative cover, minor repairs to the cap cover, and repairs and upkeep of the fence and warning signs. Major repairs may be required on occasion to address subsidence or similar threats to cap integrity, but this should not affect cap performance as long as such problems are promptly addressed. Should the cap ever be allowed 
to fail (e.g., termination of operation and maintenance), the cap would deteriorate and would not function as designed. Under this scenario, precipitation could infiltrate into treated soil; however, grouting of LAOCB soil would significantly deter any contaminant leaching from treated soil. Additionally, the clay layer (hardpan) beneath the Basin would further prevent $\mathrm{COC}$ migration.

Five year remedy reviews would be required under Alternative S-4 since contamination would remain at the waste unit above levels that would not allow for unlimited use and unrestricted exposure.

\section{Reduction of Toxicity, Mobility, or Volume}

Although in-situ S/S does not reduce the total mass and toxicity of COCs and cannot in itself achieve RGOs, it is an active form of treatment that would significantly reduce the mobility of all known soil-borne contaminants in LAOCB waste unit soil. Results of the treatability study suggest in-situ S/S will effectively reduce the mobility of primary and secondary COCs (WSRC, 1996c). When used in conjunction with capping, grouting would greatly reduce contaminant mobility, thereby reducing the inherent risk to human health and the environment. Results of the treatability study suggest that the optimal $\mathrm{S} / \mathrm{S}$ reagent is a mixture of portland cement, bentonite,

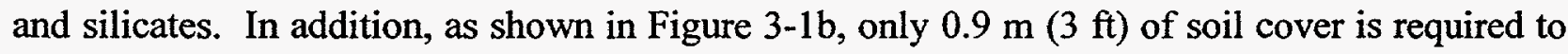
reduce the annual effective dose associated with continuous exposure to the Basin by over 99 percent. The cap would also reduce infiltration and further reduce the mobility of contaminants to unit groundwater.

\section{$\underline{\text { Short-Term Effectiveness }}$}

In-situ stabilization could be conducted using standard equipment such as trackhoes and tillermounted trackhoes or non-standard equipment such as jet grout units and augers. Depending on 
the delivery system used, the grouting rate can range from 190 to $1,150 \mathrm{~m}^{3} /$ day $(250$ to 1,500 $\mathrm{yd}^{3} /$ day; 6,750 to $40,500 \mathrm{ft}^{3} /$ day $)$. Based on the volume of soil requiring treatment $\left(760 \mathrm{~m}^{3}\right.$; $\left.1,000 \mathrm{y}^{\mathrm{d} 3}\right)$, the time required to perform the in-situ grouting would be approximately one month following mobilization. However, in consideration of potential delays due to weather and safety concerns and the capping system construction schedule, Alternative S-4 should require approximately three to five months to complete.

Under Alternative S-4, there is the potential for short-term risk to the health of remedial workers due to the handling of contaminated media. Exposure of remedial workers can be minimized and maintained well below acceptable levels with the use of proper protective clothing, scheduled monitoring of area radiation and airborne particulate levels, and strict adherence to the project health and safety plan. In addition, a layer of overburden soil would be placed over LAOCB soil to prevent airborne release of contaminants during in-situ grouting. The overburden soil would also serve as a barrier between remedial workers and contaminated soil.

Construction of a cap over the LAOCB should pose minimal risk to remedial workers. In-situ grouting of soil and cap construction should pose no significant risk to the public or the environment.

\section{Implementability}

The depth of the Basin is approximately $3.7 \mathrm{~m}(12 \mathrm{ft})$ below grade. Soil requiring remediation extend from approximately $3.7 \mathrm{~m}(12 \mathrm{ft})$ to $4.3 \mathrm{~m}(14 \mathrm{ft})$ below grade. Conventional equipment such as trackhoes or tillers mounted to trackhoes could be used to in-situ grout the LAOCB soil. Jet grouting or auger rigs that could be used to grout the Basin soil, although not considered conventional, should be available for use if selected as the delivery system. Additionally, Alternative S-4 would require the use of reagents as determined through the treatability study (e.g., Portland cement, bentonite, or silicate) and conventional earth moving equipment (e.g., 
trackhoes, backhoes, dump trucks, bulldozers, compactors) that should be readily available. Equipment decontamination would be a requirement. Materials and equipment needed for cap construction are conventional and should be readily available.

Numerous qualified contractors are available that can competitively bid on project design and construction. In-situ grouting of soil and capping are commonly used technologies that generally do not elicit public concerns.

Alternative S-4 would not preclude any future remedial actions, if warranted. For instance, disposal of LAOCB wastes at the SRS Soil Consolidation Facility could be re-evaluated should the facility become operational.

$\underline{\text { Cost }}$

Costs associated with Alternative S-4 include the labor and materials needed to in-situ grout the LAOCB soil and construct a cap over the LAOCB. Capital costs do not include any costs associated with existing institutional controls (e.g., security, groundwater monitoring). Also included in the costs is the operation and maintenance of the cap for 30 years. These costs include a review of remedy every five years for 30 years, as required by the NCP. Costs are included for comparative purposes only and are not intended to forecast actual budgetary expenditures. Estimated costs associated with Alternative S-4 are summarized below.

$\begin{array}{ll}\text { Capital Costs } & \$ 3,150,000 \\ \text { O\&M Costs } & \$ 430,000 \\ \text { Total Costs } & \$ 3,580,000\end{array}$

Costs for this alternative are provided in Appendix B. 


\subsubsection{Alternative $S$-5: Ex-Situ Stabilization and Capping}

Alternative S-5 involves the excavation and ex-situ stabilization of LAOCB soil. The primary goal of this alternative is to minimize the mobility of primary and secondary COCs. However, Alternative S-5 is an engineering approach that does not reduce the total mass of COCs and cannot achieve RGOs, it will meet remedial action objectives by reducing the leaching of contaminants to unit groundwater.

Confirmation soil samples would be collected and analyzed periodically during excavation in accordance with a confirmation sampling and analysis plan to verify that all soil exceeding concentration-based remediation goals are recovered. Prior to excavation, soil would be treated to ensure optimal waste handling characteristics. The excavated soil would be mixed with the $\mathrm{S} / \mathrm{S}$ reagents at predetermined ratios, and the soil would be placed back into the Basin. Upon completion of ex-situ grouting, the treated soil would be placed in the LAOCB, the LAOCB would be backfilled and compacted to grade, and the LAOCB would be capped as illustrated in Figures 3-2a and 3-2b. For cost estimating purposes, a compacted clay cap is assumed since all known soil-borne contamination would be confirmed treated under this alternative. The cap design would be selected during Corrective Measures/Remedial Design.

\section{Overall Protection of Human Health and the Environment}

Alternative S-5 would be protective of human health and the environment. The cap would function as a physical barrier to prevent direct human exposure to soil-borne contamination and would minimize infiltration and subsequent leaching of contamination from unsaturated soil to the groundwater. If properly constructed and maintained, caps can provide effective long-term protection of human health and the environment. Ex-situ grouting of LAOCB soil would provide an added measure of long-term protection by immobilizing all known soil-borne contamination 
at the LAOCB waste unit. Under Alternative S-5, the treatment of all known soil-borne contamination at the LAOCB waste unit would be verified by confirmation soil sampling and analysis. Therefore, Alternative S-5 meets the remedial action objective of mitigating surface exposure to contaminants and reducing the leaching of COCs to unit groundwater.

\section{Compliance with ARARs}

Chemical-specific, location-specific, and action-specific ARARs identified for Alternative S-2 would be the same as that under Alternative S-5. However, Alternative S-5 would involve treatment of all known soil-borne contamination at the LAOCB unit. Therefore, the cap included in Alternative S-5 would not be required to be a multi-layered cap design since all soil-borne contamination would be immobilized through treatment.

\section{Long-Term Effectiveness and Permanence}

Under Alternative S-5, the magnitude of residual risk remaining at the LAOCB would be low. With institutional controls and proper cap maintenance, an engineered cap would eliminate any route of direct exposure to the treated waste material. The cap would also minimize the potential for infiltration passing through the treated waste. Confirmed treatment of all known soil-borne contamination at the LAOCB waste unit would add a significant margin of safety to the effectiveness of the cap. Grouting of soil would also prove advantageous for material handling should alternative remedial actions ever be required in the future.

Cap maintenance and occasional repair would be required for as long as radiation levels posed a risk to human health. Caps have been proven effective at many waste sites and should achieve design performance standards. Existing institutional controls and scheduled maintenance would adequately maintain continued effectiveness of a cap at the LAOCB since SRS will be provided with institutional controls for the foreseeable future. Cap maintenance would include inspections 
for erosion, care for the vegetative cover, minor repairs to the cap cover, and repairs and upkeep of the fence and warning signs. Major repairs may be required on occasion to address subsidence or similar threat to cap integrity, but this should not affect cap performance as long as such problems are promptly addressed. Should the cap ever be allowed to fail (e.g., termination of operation and maintenance), the cap would deteriorate and would not function as designed; however, ex-situ grouting of LAOCB soil and the existence of the clay layer (hardpan) beneath the Basin would significantly deter contaminant leaching from LAOCB soil.

Five year remedy reviews would be required under Alternative S-5 since contamination would remain at the waste unit above levels that would not allow for unrestricted use and unrestricted exposure.

\section{Reduction of Toxicity, Mobility, or Volume}

Although ex-situ S/S does not reduce the total mass and toxicity of COCs and cannot in itself achieve RGOs, it is an active form of treatment that would significantly reduce the mobility of all known soil-borne contaminants in LAOCB waste unit soil. Results of the treatability study suggest ex-situ S/S will effectively reduce the mobility of primary and secondary COCs (WSRC, 1996c). When used in conjunction with capping, grouting would greatly reduce contaminant mobility, thereby reducing the inherent risk to human health and the environment. Results of the treatability study suggest that the optimal S/S reagent is a mixture of portland cement, bentonite, and silicates. In addition, as shown in Figure $3-1 b$, only $0.9 \mathrm{~m}(3 \mathrm{ft})$ of soil cover is required to reduce the annual effective dose associated with continuous exposure to the Basin by over 99 percent. The cap would also reduce infiltration and further reduce the mobility of contaminants to unit groundwater. One disadvantage of $S / S$ technologies is that the volume of contaminated media may increase; however, the treated media may be wholly contained within the LAOCB. Therefore, the overall area and volume of waste to be managed would not increase. 


\section{$\underline{\text { Short-Term Effectiveness }}$}

Under Alternative S-5, there is the potential for short-term risk to the health of remedial workers due to the extensive handling of contaminated media. The volume of contaminated media directly handled by remedial workers would be approximately $760 \mathrm{~m}^{3}\left(27,000 \mathrm{ft}^{3} ; 1,000 \mathrm{yd}^{3}\right)$ of contaminated soil. Care would be required to minimize the generation of airborne particulates during processing. This could be accomplished by applying dust suppressants, monitoring for airborne particulates, and strict adherence to project work plans. Remedial worker exposure can be minimized and maintained within acceptable levels with the use of proper protective clothing, scheduled monitoring of area radiation and airborne particulate levels, and strict adherence to the project health and safety plan.

Construction of a cap over the LAOCB should pose minimal short-term risk to remedial workers. Ex-situ grouting of soil and cap construction should pose no significant short-term risk to the public or the environment. Erosion control measures would be taken to minimize the potential for erosion in areas where soil would be removed.

Ex-situ grouting should take approximately one month to complete after mobilization to the field. This assumes a soil processing rate of $150 \mathrm{~m}^{3}\left(5,400 \mathrm{ft}^{3} ; 200 \mathrm{yd}^{3}\right)$ per day. The actual processing rate will vary according to the type of S/S reagent used, as well as the number of processing units employed. Additionally, potential delays could occur during implementation due to weather and safety concerns. With construction of the capping system, Alternative S-5 should require approximately three to five months to complete.

Implementability

According to unit-specific treatability study results, Alternative S-5 would require readily available reagents as determined through the treatability study (e.g., Portland cement, bentonite, 
or silicate), mixing equipment, and the use of conventional earth moving equipment (e.g., backhoes, dump trucks, bulldozers, compactors). Extensive decontamination of equipment would be required since the process would involve significant materials handling and processing. Numerous qualified contractors should be available that could competitively bid on project design and construction.

Materials and equipment needed for cap construction are conventional and should be readily available. Ex-situ stabilization of soil and capping are commonly used technologies that generally do not elicit public concerns.

Alternative S-5 would not preclude any future remedial actions, if warranted. For instance, disposal of LAOCB wastes at the SRS Soil Consolidation Facility could be re-evaluated should the facility become operational.

\section{$\underline{\text { Cost }}$}

Costs associated with Alternative S-5 include the labor and materials needed to pre-treat the soil, to excavate the soil and ex-situ stabilize the LAOCB soil and construct a cap over the LAOCB. Due to the direct handling and processing of contaminated soil, an assumed standby time is included in estimated costs to account for potential work delays that may be required to address health and safety issues. Capital costs do not include any costs associated with existing institutional controls (e.g., security, groundwater monitoring). Also included in the costs is the operation and maintenance of the cap for 30 years. These costs include a review of remedy every five years for 30 years, as required by the NCP. Costs are provided for comparative purposes only and are not intended to forecast actual budgetary expenditures. Estimated costs associated with Alternative S-5 are summarized below.

$\begin{array}{ll}\text { Capital Costs } & \$ 3,940,000 \\ \text { O\&M Costs } & \$ 430,000 \\ \text { Total Costs } & \$ 4,370,000\end{array}$


Costs for this alternative are provided in Appendix B.

\subsubsection{Alternative S-6: Excavation and Disposal at the NTS Facility}

Alternative S-6 involves the excavation and off-unit disposal of all known soil-borne contamination at the LAOCB waste unit. With the exception of restrictions on groundwater use, the LAOCB could be released for unrestricted use under this alternative since all contaminated soil known to exceed concentration-based remediation goals (background levels) would be permanently removed from the waste unit.

Treatment of the LAOCB soil would first be conducted to ensure optimal waste handling characteristics. Following pre-treatment a backhoe or trackhoe would be used to excavate contaminated LAOCB soil. Confirmation soil samples would be collected and analyzed periodically during excavation in accordance with a confirmation sampling and analyses plan to verify that all soil exceeding concentration-based remediation goals were recovered. Following excavation, the soil may require further treatment for waste handling purposes and packaging and disposal requirements. Soil would then be placed directly into lined haul trucks for transport from the waste unit. From the LAOCB, the excavated soil would be transported directly by truck or intermodal by both rail and truck to the NTS facility near Mercury, Nevada. When soil removal and disposal are completed, the LAOCB excavation would be backfilled and compacted to grade. Capping of the LAOCB would not be warranted under these alternatives since all known soil-borne contamination would be removed from the LAOCB waste unit.

\section{Overall Protection of Human Health and the Environment}

Alternative S-6 would be protective of human health and the environment. Under Alternative S6 , all routes of exposure to contaminated soil would be permanently eliminated from the LAOCB 
waste unit and LAOCB soil would no longer be a source of contamination to the environment. Therefore, removal and disposal of the contaminated soil at the NTS facility would achieve both protectiveness of human health and the environment. Therefore, Alternative S-6 meets the remedial action objectives by achieving RGOs, reducing risk to human health and the environment, and mitigating the release of COCs to unit groundwater.

\section{Compliance with ARARs}

All chemical-specific ARARs discussed under Alternative S-2 (Section 4.2.2) would be applicable to Alternative S-6. Alternative S-6 would meet the ARARs and to-be-considered information discussed. Location-specific ARARs identified for Alternative S-2 ('Section 4.2.2) would be the same as that under Alternative S-6.

In addition to the action-specific ARARs and TBC information identified under Alternative S-2 (Section 4.2.2); Alternative S-6 could include regulatory requirements associated with the transport and treatment of radioactive materials. The soil removed under Alternative S-6 would be transported to the NTS facility for disposal. Transport of radioactive materials would be regulated under the Department of Transportation (DOT) regulations promulgated under 49 CFR Parts 172 through 203. Transport of radioactive materials within the boundaries of SRS must also be as specified in DOE Order 5480.3: Safety Requirements for the Packaging and Transportation of Hazardous Materials, Hazardous Substances, and Hazardous Wastes.

\section{Long-Term Effectiveness and Permanence}

Under Alternative S-6, the magnitude of residual risk remaining at the LAOCB would be minimal. Remaining soil would not be contaminated, or would contain contaminants at levels below conservative concentration-based remediation goals. Consequently, remaining soil would not pose unacceptable risks to human health or the environment. 
Five year remedy reviews would not be required under Alternative S-6 since contamination would not be present at the waste unit at levels above concentration-based remediation goals and, with the exception of groundwater use, the LAOCB could be released for unrestricted land use.

\section{Reduction of Toxicity, Mobility, or Volume}

Alternative S-6 would involve no treatment of soil that would result in a reduction of contaminant toxicity, mobility, or volume.

\section{Short-Term Effectiveness}

Excavation and disposal of contaminated soil are relatively straight-forward processes; however, due to the handling of contaminated media, there is the potential for short-term risk to the health of remedial workers. The estimated volume of contaminated media handled by remedial workers would be approximately $760 \mathrm{~m}^{3}\left(27,000 \mathrm{ft}^{3} ; 1,000 \mathrm{yd}^{3}\right)$ of LAOCB soil. Exposure to remedial workers can be minimized and maintained well below acceptable levels with the use of proper protective clothing, scheduled monitoring of area radiation and airborne particulate levels, and strict adherence to the project health and safety plan.

LAOCB soil would be transported to the NTS facility approximately $3,500 \mathrm{~km}(2,200 \mathrm{mi})$ away. Although transport of contaminated soil from the LAOCB waste unit would increase the risk for exposure to contaminated materials, scheduling, road selection, and transport notifications would adequately reduce the risk of unwarranted exposure and accidents. The disposal process should pose minimal risk to disposal facility operators, the community, and the environment.

Alternative S-6 should pose no significant risk to the environment. Erosion control measures would be taken to minimize the potential for erosion in areas where soil would be removed. 
Alternative S-6 should take approximately one month to complete after mobilization to the field. This assumes that soil are removed at a rate of $138 \mathrm{~m}^{3}\left(4,860 \mathrm{ft}^{3} ; 180 \mathrm{yd}^{3}\right)$ per day. Actual soil removal rates would be determined during Corrective Measures/Remedial Design. In consideration of potential delays due to weather and safety concerns, the implementation schedule could extend to approximately two months.

Implementability

Alternative S-6 should be implementable; however, extensive waste handling is required. The NTS facility is certified to accept low-level radioactive wastes from DOE facilities. No future remedial actions would be evaluated following implementation of Alternative S-6.

\section{$\underline{\text { Cost }}$}

Costs associated with Alternative S-6 include the labor and materials needed to excavate, transport, and dispose of contaminated LAOCB soil at the NTS facility. Due to the direct handling of contaminated soil, an assumed standby time is included in estimated costs to account for potential work delays that may be required to address health and safety issues. Costs are included for comparative purposes only and are not intended for forecasting budgetary expenditures. Estimated costs associated with Alternative S-6 are summarized below.

$\begin{array}{ll}\text { Capital Costs } & \$ 9,100,000 \\ \text { O\&M Costs } & \frac{\$}{\$ 9,100,000}\end{array}$

Costs for Alternative S-6 are provided in Appendix B. 


\subsubsection{Comparative Analysis}

Following is a comparative analysis of the six remedial alternatives being considered for remediating LAOCB contaminated soil. Comparisons are summarized in Table 4-1. LAOCB remedial alternatives are evaluated against the NCP threshold (overall protection of human health, compliance with ARARs) and primary balancing criteria (long-term effectiveness and permanence; reduction of toxicity, mobility, or volume; short-term effectiveness; implementability; and cost).

\subsubsection{Overall Protection of Human Health and the Environment}

The No Action alternative would be protective of human health as long as institutional controls are maintained. The existence of the clay layer (hardpan) beneath the Basin adequately prevents the migration of COCs, through the vadose zone thereby protecting groundwater. The No Action alternative, however, provides no means of verifying whether contaminants would impact groundwater in the future.

Remaining alternatives being considered would all be protective of human health and the environment. With the exception of Alternative S-6 (Disposal at NTS), all other alternatives would involve capping, which would (1) act as a barrier that would deter human access to contaminated media, (2) minimize infiltration and leaching of contaminants from soil to groundwater, (3) act as shielding to reduce radiation exposure to hypothetical receptors to within acceptable levels, and (4) serve as redundant protective feature for those alternatives that involve treatment as a primary means of remediating contaminated soil. 


\subsubsection{Compliance with ARARs}

The chemical-specific ARARs associated with the LAOCB include concentration-based standards for $\mathrm{Ra}$ and $\mathrm{Th}$ in surface and subsurface soil specified in UMTRCA. Sediment samples were not analyzed for alpha emitting Ra isotopes. Therefore, it is unknown whether the level of $\mathrm{Ra}$ in soil at the LAOCB waste unit meets the identified ARAR. No detectable activities of ${ }^{230} \mathrm{Th}$ or ${ }^{232} \mathrm{Th}$ were present in the Basin sediment.

EPA regulation 40 CFR 192 and DOE Order 5400.5 are considered relevant and appropriate and to-be-considered information, respectively. The EPA standard specifies an allowable annual effective dose to any member of the public resulting from nuclear power plant operations. The allowable effective dose rate is $25 \mathrm{mrem} / \mathrm{year}$. The DOE Order specifies an allowable annual effective dose to any member of the public resulting from all DOE operations of $100 \mathrm{mrem} / \mathrm{year}$. With the exception of the No Action alternative (Alternative S-1), an evaluation of remaining alternatives using very conservative assumptions indicates that implementation of the alternatives would meet the allowable effective dose rates under 40 CFR 192 and DOE Order 5400.5. Should institutional controls be maintained at SRS for 166 years, the No Action alternative would meet the regulatory guidelines.

Action-specific ARARs identified for the evaluated alternatives are generally similar; however, no ARARs are identified for the No Action alternative. All remaining alternatives require NESHAPs air modeling, county erosion control plans, and OSHA health and safety plans. RCRA capping performance standards are required for all alternatives except No Action and off-unit disposal. Alternative S-6 requires transportation of radioactive materials within SRS boundaries and off site to the NTS facility, which would require adherence to DOE Order 5480.3 and 49 CFR 172 through 203. 


\subsubsection{Long-Term Effectiveness and Permanence}

Long-term effectiveness and permanence can be measured in broad terms by (1) the magnitude of residual risks associated with the waste unit, and (2) the adequacy of controls after implementation of the remedial alternative. Of the alternatives being considered, the No Action alternative is the least effective alternative in terms of the magnitude of residual risks after implementation since it would leave all contaminated media in place without the benefit of treatment. Alternatives S-2 and S-3, which involve the capping of all contaminated media and vertical barriers, would significantly reduce the magnitude of residual risks over No Action since they would minimize infiltration reaching the waste; however, Alternatives S-2 (Capping) and S3 (Capping and Slurry Wall) do not involve any form of treatment that would permanently reduce the magnitude of residual risk. With the exception of No Action (Alternative S-1) and Alternative S-6 (Disposal at NTS), all other alternatives involve capping and treatment of contaminated media. Alternative S-6 involves off-unit disposal of all contaminated soil above concentration-based remediation goals but does not involve capping. Alternatives S-4 (In-Situ Stabilization), S-5 (Ex-Situ Stabilization), and S-6 (Disposal at NTS) offer a greater reduction in the magnitude of residual risks than would Alternatives S-2 (Capping) and S-3 (Capping and Slurry Wall).

Alternatives S-4 (In-Situ Stabilization) and S-5 (Ex-Situ Stabilization) involve some form of treatment that would permanently reduce the magnitude of on-unit residual risks by reducing contaminant mobility and/or volume. Alternative S-6 involves no form of treatment to reduce the magnitude of residual risk associated with contaminated media; however, this alternative involves the disposal of contaminated soil at the NTS facility and would effectively remove all residual risk at the unit.

With respect to contaminated soil, Alternative S-6 (Disposal at NTS) offers the greatest reduction in residual risk since it would permanently remove all contaminated soil at concentrations above 
concentration-based remediation goals from the LAOCB waste unit. Residual concentrations left in soil would not pose a significant risk to human health or the environment. Alternatives S-4 (In-Situ Stabilization) and S-5 (Ex-Situ Stabilization) would immobilize soil-borne contaminants. The residual risks associated with Alternative S-5 would be slightly less than that of Alternative S-4 because the treatment of all known soil-borne contamination at the LAOCB waste unit would be verified by confirmation sampling under Alternative $S-5$, whereas treatment of all known soil-borne contamination would not be confirmed under Alternative S-4.

Existing SRS institutional controls would be adequate for the protection of human health as long as the institutional controls are maintained. In the absence of existing controls, the No Action alternative would not be protective of human health. Based upon the hypothetical scenario that institutional controls cannot be guaranteed and/or proposed caps could be allowed to fail, the need for controls to maintain protectiveness would decrease corresponding to the extent to which contaminated media are treated to permanently reduce the magnitude of residual risks. Consequently, the need for controls is greatest for alternatives that do not treat or remove any of the contaminated media (Alternatives S-1 - No Action, S-2 - Capping, and S-3 - Capping and Slurry Wall) followed by alternatives that treat all known contaminated soil at the LAOCB waste unit (Alternatives S-4 - In-Situ Stabilization and S-5 - Ex-Situ Stabilization). Alternative S-6 (Disposal at NTS) would require the least controls of all alternatives being considered since it. would involve the permanent removal of all contaminated soil known to exceed concentrationbased remediation goals. With the exception of restrictions on groundwater use, no controls would be required for the LAOCB waste unit under Alternative S-6.

\subsubsection{Reduction of Toxicity, Mobility, or Volume}

Alternatives S-1 (No Action), S-2 (Capping), S-3 (Capping and Slurry Walls), and S-6 (Disposal at NTS) offer no form of active treatment and, therefore, do not satisfy the NCP preference for remedial alternatives that offer a reduction in contaminant toxicity, mobility, or volume. All 
other alternatives being considered offer some form of active treatment that would permanently reduce contaminant toxicity, mobility, or (contaminated media) volume. The treatment technology being considered for treating LAOCB contaminated soil is stabilization/solidification by grouting (soil only), which reduces contaminant mobility.

\subsubsection{Short-Term Effectiveness}

The short-term risks to remedial workers increases with the volume of contaminated media directly handled or processed and project duration. Handling (e.g., excavating, moving) and/or processing (e.g., treating) contaminated media increases the risk of remedial worker exposure to radiation effects. In addition, remedial workers are exposed to potential construction-related risks (e.g., falls, cuts, heavy equipment operation) which increase with corresponding increases in project duration; however, potential short-term risks to remedial workers should be manageable for all alternatives being considered. With strict adherence to project health and safety plans, it should be possible to maintain short-term risks of all considered alternatives within acceptable limits.

The potential risk to remedial workers would be lowest for the No Action alternative, followed by Alternative S-2 (Capping) which involves no or very limited handling or processing of contaminated media. The No Action alternative requires no time in the field, whereas the estimated time to complete Alternative S-2 (Capping) is four months once fieldwork begins. Alternative S-6 (Disposal at NTS) would not require an extensive timeframe to complete the remediation beyond that of Alternative S-2.

The alternatives posing the greatest potential risks to remedial workers would be Alternatives S-5 (Ex-Situ Stabilization) and S-6 (Disposal at NTS) because they involve the direct handling and processing of the greatest volume of contaminated media. Standby time would be anticipated for these alternatives to address health and safety issues since these alternatives would involve 
extensive handling and/or processing of contaminated soil. Work stoppages would significantly impact the time needed to complete these alternatives. After mobilization to the field, the estimated time to complete Alternatives S-5 and S-6 is two months.

All alternatives being considered would pose negligible or very low risks to the community. Alternative S-6 involves transport of contaminated soil to the NTS facility near Mercury, Nevada, 3,500 km (2,200 mi) from the LAOCB waste unit. Under Alternatives S-1 (No Action), S-2 (Capping), S-3 (Capping and Slurry Walls), S-4 (In-Situ Stabilization), and S-5 (Ex-Situ Stabilization), the risks posed to the community would be negligible since they would not include off-unit transport of contaminated media.

\subsubsection{Implementability}

Alternative S-1 (No Action) would be the most implementable alternative being considered since it would not involve any type of construction or remedial actions beyond existing institutional controls; however, the No Action alternative could potentially arouse public concern since it would pose a potential threat to the environment. Alternative S-2 (Capping) would involve the construction of a cap, but should be relatively easy to implement. Alternative S-3 would involve the construction of slurry cut-off walls and a cap, both of which are readily constructed. Alternatives S-2 and S-3 should not elicit major public concerns. Alternatives S-4 (In-Situ Stabilization) and S-5 (Ex-Situ Stabilization) should be implementable; however, Alternative S-5 requires extensive waste handling and pre-excavation treatment of soil. Stabilization and disposal are commonly applied technologies for remediating low-level or mixed wastes, and should not elicit public concerns. Alternative S-6 (Disposal at NTS) would be readily implementable; however, there may be potential public concern regarding the off-site transportation of low-level or mixed wastes. Alternative S-6 also would require treatment of soil prior to excavation to ensure optimal waste handling characteristics. Post-excavation treatment may be needed under Alternative S-6 for packaging and disposal requirements. 
With the exception of Alternative S-6, future remedial alternatives, if warranted, would not be precluded by implementing any of the alternatives. Disposal of LAOCB wastes at the SRS Soil Consolidation Facility, for instance, could be re-evaluated should the facility become operational in the future.

\subsubsection{7 $\underline{\text { Cost }}$}

Total estimated present worth costs range between $\$ 280,000$ for the No Action alternative to $\$ 9,100,000$ for Alternative S-6 (Disposal at NTS). Alternative S-2 $(\$ 1,430,000)$ involves capping only. Alternative S-3 $(\$ 3,430,000)$ involves slurry cut-off walls. Alternative S-4 $(\$ 3,580,000)$ involves in-situ stabilization the contaminated soil. Alternative $S-5(\$ 4,370,000)$ involves ex-situ stabilization the contaminated soil. Alternative S-6 $(\$ 9,100,000)$ involves off-

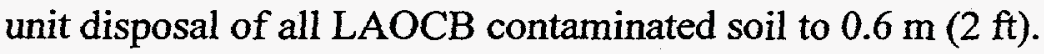

With the exception of Alternatives S-1 and S-6, the estimated operation and maintenance costs of all alternatives are approximately $\$ 430,000$ for the long-term (30 years) maintenance of a cap. The estimated operation and maintenance for the No Action alternative (Alternative S-1) is $\$ 280,000$ because it does not involve capping. Alternative S-6 would have no additional operation and maintenance costs above the No Action alternative since it would permanently remove all contaminated soil from the LAOCB waste unit. All cost estimates are provided for comparison purposes only and are not intended to forecast actual budgetary expenditures. Cost estimates are provided in Appendix B.

\subsection{Individual Analysis Of Pipeline Alternatives}

Remedial alternatives for the LAOCB contaminated pipelines are evaluated against the threshold and primary balancing criteria in the following sections. 


\subsubsection{Alternative P-1: No Action}

Under the No Action alternative, no remedial efforts would be conducted to remove, treat, or otherwise lessen the toxicity, mobility, or volume of contamination within the LAOCB pipelines. Existing SRS institutional controls would likely be maintained in the foreseeable future, but would not be guaranteed under the No Action alternative. Radioactive decay would provide a reduction in radioisotopes present in the pipelines. However, since radionuclide species and activities have not been identified, the time required for radioactive decay within acceptable levels is unknown

\section{Overall Protection of Human Health and the Environment}

To date, radioisotopes have not been detected in pipeline soils or groundwater from the pipeline areas. This suggests that radioisotopes are not leaking from the pipelines. Without institutional controls to prohibit access to the pipeline areas, the potential exists for human or wildlife intrusion and subsequent exposure to the pipelines. Furthermore, based on the shallow depth of

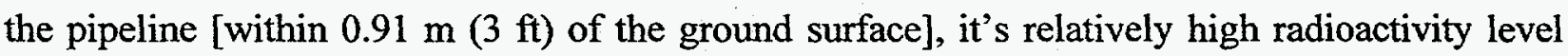
[approximately 300,000 disintegrations per minute $(\mathrm{dpm})$ on internal surface], the age $(>30$. years) and material of the pipe (cast iron which could degrade over time and release radioisotopes), Alternative P-1 (No Action) would not be protective of human health or the environment. 


\section{Compliance with ARARs}

\section{Chemical-Specific ARARs}

The only nonradioactive chemical-specific ARARs which may be applicable under Federal and South Carolina regulations are for PCBs. ARARs for PCBs are governed by TSCA (40 CFR Part 761). Based on the disposal history of the LAOCB, no PCBs are expected.

The only regulation that may be applicable to the LAOCB pipelines is a TBC factor. DOE Order 5400.5 - Radiation Protection of the Public and the Environment which regulates the effective dose received as a result of radiation. The pipelines were not sampled for specific isotopes. Therefore, it is unknown whether the radiation levels resulting from radioisotopes in the pipelines would meet the TBC specified annual dose equivalents (which are discussed further in Section 2.3.2.1). Considering that $0.9 \mathrm{~m}$ to $1.2 \mathrm{~m} \mathrm{(3} \mathrm{to} 4 \mathrm{ft}$ ) of soil cover the pipelines, it is unlikely that gamma radiation exceeds the TBC annual dose equivalents. However, compliance with this potential ARAR cannot be evaluated for Alternative P-1.

\section{Location-Specific ARARs}

No location-specific ARARs are associated with the No Action alternative.

Action-Specific ARARs

No action-specific ARARs are associated with the No Action alternative. 


\section{Long-Term Effectiveness and Permanence}

Based on the shallow depth of the pipeline [within $0.91 \mathrm{~m}(3 \mathrm{ft})$ of the ground surface], it's relatively high radioactivity level (approximately $300,000 \mathrm{dpm}$ on internal surface), the age $(>30$ years) and material or the pipe (cast iron which could degrade over time and release radioisotopes), long-term protectiveness of human health can not be guaranteed.

\section{Reduction of Toxicity, Mobility, or Volume}

The No Action alternative would not involve any form of active treatment that would reduce contaminant or contaminated media toxicity, mobility, or volume. However, ongoing radioactive decay reduces the toxicity and volume of contaminants.

\section{Short-Term Effectiveness}

Based on current conditions and existing institutional controls, exposure to LAOCB pipelines would be prevented. In addition, Alternative P-1 would not involve any construction activities which would endanger public communities, remedial workers, or adversely impact the environment.

\section{Implementability}

The No Action alternative would require no construction, specialized equipment, or technical specialists and could, therefore, be implemented immediately. Access to the LAOCB and surrounding areas would be controlled through the designated custodian. If implemented, No Action may prompt public concern for protection of human health and the environment. Alternative P-1 would not preclude any future remedial actions, if warranted. 
$\underline{\text { Cost }}$

The only costs associated with the No Action alternative would be a review of remedy every five years for 30 years, as required by the NCP. No long-term operation and maintenance costs would be associated with the No Action alternative. Remedy review costs $(\$ 280,000)$ would be included for the LAOCB OU, dependent upon the remedy selected for the LAOCB.

\section{Controls}

\subsubsection{Alternative P-2: Capping}

Alternative P-2 consists of construction of a cap over the LAOCB pipeline areas. Initially, the waste unit would be prepared by clearing any vegetation, fencing, and other physical obstructions immediately surrounding the pipelines. After sufficient compaction, an engineered cap would be constructed over the pipeline areas. The cap would cover each pipeline and would

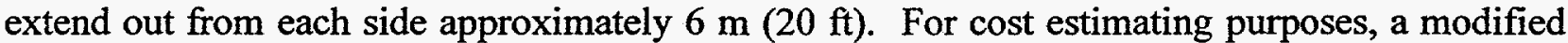
RCRA cap is assumed for this alternative since it would allow untreated contaminants to remain in place. Cap design would be evaluated and selected during detailed design. Existing SRS institutional controls would remain in effect and the capping system would be maintained for a minimum of 30 years.

\section{Overall Protection of Human Health and the Environment}

With proper design, construction, and maintenance, Alternative P-2 would be protective of human health and the environment. The cap would function as a physical barrier to prevent direct human and wildlife access and exposure to contamination present in the pipelines. The cap would also minimize infiltration, thereby providing further protection from radionuclide migration to unit groundwater. Therefore, Alternative P-2 meets the remedial action objective of 
reducing risk to human health and the environment and reducing the leaching of COCs to unit groundwater.

\section{Compliance with ARARs}

Chemical-Specific ARARs

The discussion of the TSCA and ARARs identified under the No Action alternative also applies to Alternative P-2. However, in regards to the public allowable effective dose equivalent specified in DOE Order 5400.5, capping of the pipelines could reduce the effective dose equivalent to within acceptable limits. Therefore, Alternative P-2 would achieve chemicalspecific ARARs.

\section{Location-Specific ARARs}

No location-specific ARARs are associated with Alternative P-2.

\section{Action-Specific ARARs}

The capping system for Alternative P-2 would likely consist of a multi-layered RCRA cap. The particular design of the capping system would be selected during Remedial Design. A multilayered RCRA cap must meet the performance standards of RCRA (40 CFR 264.310) as follows:

- provide long-term minimization of migration of liquids

- $\quad$ function with minimum maintenance

- $\quad$ promote drainage and minimize erosion or abrasion of the cover

- accommodate settling and subsidence to maintain cover integrity

- have a permeability less than that of natural subsurface soil 
Numerous capping system designs would meet the RCRA performance standards; therefore, no difficulties can be perceived for designing a capping system under Alternative P-2.

Workers performing the remediation at the LAOCB would be required to adhere to a project health and safety plan specified under the Occupational Safety and Health Association (OSHA; 29 CFR 1910).

\section{Long-Term Effectiveness and Permanence}

Long-term protectiveness of human health would be achieved under Alternative $\mathrm{P}-2$ because it would restrict access of humans and wildlife to the LAOCB pipelines and minimize the potential for migration of pipeline contaminants to unit groundwater. Cap maintenance would be required and would include minor repairs to the cap cover and repairs and inspection and upkeep of the warning signs. Major repairs may be required on occasion to address subsidence or similar threats to cap integrity, but this should not affect cap performance as long as such problems are promptly addressed.

\section{Reduction of Toxicity, Mobility, or Volume}

Alternative P-2 would not involve any form of treatment that would reduce the toxicity, mobility, or volume of pipeline contaminants; however, capping would effectively reduce contaminant mobility by minimizing infiltration and potential contaminant leaching to unit groundwater. Additionally, natural radioactive decay would decrease the toxicity and volume of radioactive contamination. 


\section{Short-Term Effectiveness}

In general, cap construction is a straight-forward process requiring minimal disturbance of contaminated media. Although short-term risks to remedial workers would exist, the risks should be manageable. Exposure to remedial workers can be minimized and maintained well below acceptable levels with the use of proper protective clothing, scheduled monitoring of area radiation and airborne particulate levels, and strict adherence to the project health and safety plan. Consequently, short-term risks to the health of remedial workers would be minimal. Because there would not be any disturbance of the pipelines, Alternative P-2 would not pose a short-term risk to the community or environment. Alternative P-2 should take approximately two months to complete after mobilization to the field.

\section{Implementability}

Alternative P-2 would be readily implementable. Caps have been commonly used at other lowlevel radioactive and mixed waste sites and generally do not elicit public concerns. Depending upon the cap design selected, Alternative 2 would require readily available materials (e.g., topsoil, clay, gravel, geosynthetics) and the use of conventional earth moving equipment (e.g., backhoes, dump trucks, bulldozers, compactors). Numerous qualified contractors are available that can competitively bid on the design and construction of an engineered cap.

Routine monitoring would be conducted to verify remedy effectiveness. Periodic monitoring of groundwater quality and cap subsidence would be conducted as part of ongoing institutional controls. Alternative P-2 would not preclude any future remedial actions, if warranted. 


\section{$\underline{\text { Cost }}$}

Costs associated with Alternative P-2 include the labor and materials needed to construct the cap and operate and maintain the remedy for 30 years. Operation and maintenance would include mowing and minor repairs to the cap and fence. A review of remedy every five years for 30 years is required by the NCP; however, the remedy review cost $(\$ 280,000)$ would be included with that of the LAOCB OU, depending on the remedy selected for the LAOCB. Costs are provided for comparison purposes only and are not intended to forecast actual budgetary expenditures. Estimated costs associated with Alternative P-2 are summarized below. Detailed costs for Alternative P-2 are provided in Appendix B.

$\begin{array}{ll}\text { Capital Costs } & \$ 560,000 \\ \text { O\&M Costs } & \$ 170,000 \\ \text { Total Costs } & \$ 730,000\end{array}$

\subsubsection{Alternative P-3: In-situ S/S, Excavation and Disposal in the LAOCB}

Under Alternative P-3, the pipelines would be grouted, excavated, downsized by cutting, and disposed of in the LAOCB. The LAOCB has the capacity to accommodate disposal of the pipelines and associated contaminated materials once the pipe has been downsized. Following the removal of all pipeline and associated contaminated materials, the pipeline area would be backfilled to grade, and would be disposed in the pipelines in the LAOCB. After the grouted pipelines are excavated and disposed of inside the LAOCB, the remaining void space in the basin would be grouted with the same mixture used for in-situ S/S. Grouting around the pipeline sections inside the basin would further encapsulate the outside of the pipelines, and create a monolith that would further reduce the mobility of the pipeline contaminants. Under this alternative the LAOCB pipeline areas could be released for unrestricted use since contaminated media would be permanently removed. 
The LAOCB pipelines would be grouted and excavated using conventional equipment. Confirmation soil samples would be collected and analyzed periodically during excavation in accordance with a confirmation sampling and analyses plan to verify that all contaminated material is recovered. Following excavation, the pipelines would be downsized by cutting and then disposed of in the LAOCB. When all contaminated material is removed and disposal activities are completed, the excavation area would be backfilled and compacted to grade.

\section{Overall Protection of Human Health and the Environment}

Alternative P-3 would be protective of human health and the environment since all routes of exposure to contaminated soil would be permanently eliminated from the LAOCB pipeline area. Therefore, Alternative P-3 meets the remedial action objective of reducing risk to human health and the environment and mitigating the leaching of COCs to unit groundwater.

\section{Compliance with ARARs}

All chemical-specific ARARs discussed under Alternative P-3 would be applicable to Alternative P-3. Alternative P-3 would meet the TBC factor discussed since all contamination would be removed from the pipeline areas. No location-specific or action-specific ARARs were. identified for Alternative P-3.

\section{Long-Term Effectiveness and Permanence}

Under Alternative P-3, the magnitude of residual risk remaining at the LAOCB pipeline areas would be negligible. Five year remedy reviews would not be required under Alternative P-3 since contamination would not be present at the waste unit and the LAOCB pipeline areas could be released for unrestricted land use. 
Reduction of Toxicity, Mobility, or Volume

In-situ S/S (grouting of the pipelines) is an active form of treatment that would significantly reduce the mobility of residual pipeline contaminants. Grouting before excavation and removal would greatly reduce contaminant mobility, thereby reducing the potential release of residual contaminants from inside the pipeline to the environment. After the grouted pipelines are excavated and disposed of inside the LAOCB, the remaining void space in the basin would be grouted to encapsulate the pipeline sections inside the basin and create a monolith that would further reduce the mobility of the pipeline contaminants.

\section{Short-Term Effectiveness}

Excavation and disposal of contaminated pipelines and soil are relatively straight-forward processes; however, due to the extensive handling of contaminated media, there could be significant short-term risk to the health of remedial workers. However, exposure to remedial workers could be minimized and maintained below acceptable levels with the use of proper protective clothing, scheduled monitoring of area radiation and airborne particulate levels, and strict adherence to the project health and safety plan. The LAOCB pipelines would be downsized by cutting and then transported to the LAOCB which is directly adjacent to the pipelines. The disposal process should not pose any additional risk to remedial workers.

Alternative P-3 should pose only a minimal risk to the environment. Erosion control measures would be taken to minimize the potential for erosion in areas where soil would be moved. Alternative P-3 should take approximately two months to complete after mobilization to the field. 


\section{Implementability}

Alternative P-3 should require conventional grouting and excavation equipment; however, extensive waste handling is required. No future remedial actions would be evaluated following implementation of Alternative P-3. Alternative P-3 could be readily implemented.

\section{$\underline{\text { Cost }}$}

Costs associated with Alternative P-3 include the labor and materials needed to excavate and dispose of the LAOCB pipelines in the LAOCB. Due to the direct handling of the pipelines, an assumed standby time is included in estimated costs to account for potential work delays that may be required to address health and safety issues. Because disposal is a permanent solution, there are no long-term operation and maintenance costs associated with this alternative. Costs are included for comparative purposes only and are not intended for forecasting budgetary expenditures. Estimated costs associated with Alternative P-3 are summarized below. Detailed costs for Alternative P-3 are provided in Appendix B.

\begin{tabular}{lr} 
Capital Costs & $\$ 990,000$ \\
O\&M Costs & $\$ \quad 0$ \\
\hline Total Costs & $\$ 990,000$
\end{tabular}

\subsubsection{Alternative P-4: Excavation and Disposal at the NTS Facility}

Under Alternative P-4, the pipelines would be grouted, excavated, downsized by cutting, and subsequently transported to the NTS for disposal. Following the removal of all pipeline and associated contaminated materials, the pipeline area would be backfilled to grade and made available for future use. 
The LAOCB pipelines would be grouted and excavated using conventional equipment. Confirmation soil samples would be collected and analyzed periodically during excavation in accordance with a confirmation sampling and analyses plan to verify that all contaminated material is recovered. Following excavation, the soil may require further treatment for waste handling purposes and packaging and disposal requirements. The pipelines would likely be downsized by cutting and placed into roll-off boxes and then loaded on trucks for transport from the waste unit. From the LAOCB, the pipelines would be transported directly by truck or intermodal by both rail and truck to the NTS facility near Mercury, Nevada. When the pipeline removal and disposal are completed, the excavation areas would be backfilled and compacted to grade.

Overall Protection of Human Health and the Environment

Alternative P-4 would be protective of human health and the environment since all routes of exposure to the pipelines would be permanently eliminated from the LAOCB pipeline area. Alternative P-4 meets the remedial action objectives by achieving RGOs, reducing risk to human health and the environment, and mitigating the leaching of COCs to unit groundwater.

\section{Compliance with ARARs}

All chemical-specific ARARs discussed under Alternative P-2 would be applicable to Alternative P-4. Alternative P-4 would meet the TBC factor discussed since all contamination would be removed from the pipeline areas. No location-specific ARARs were identified for Alternative P-4. Action-specific ARARs that would apply to Alternative P-4 would include regulatory requirements associated with the transport and treatment of radioactive materials. The pipelines removed under Alternative P-4 would be transported to the NTS facility for disposal. Transport of radioactive materials would be regulated under the Department of Transportation (DOT) regulations promulgated under 49 CFR Parts 172 through 203. Transport of radioactive 
materials within the boundaries of SRS must also be as specified in DOE Order 5480.3: Safety Requirements for the Packaging and Transportation of Hazardous Materials, Hazardous Substances, and Hazardous Wastes.

\section{Long-Term Effectiveness and Permanence}

Under Alternative P-4, the magnitude of residual risk remaining at the LAOCB pipeline areas would be negligible. Five year remedy reviews would not be required under Alternative P-4 since contamination would not be present at the waste unit and the LAOCB pipeline areas could be released for unrestricted land use.

\section{Reduction of Toxicity, Mobility, or Volume}

In-situ $\mathrm{S} / \mathrm{S}$ (grouting of the pipelines) is an active form of treatment that would significantly reduce the mobility of residual pipeline contaminants. Grouting before excavation and removal would greatly reduce contaminant mobility, thereby reducing the potential release of residual contaminants from inside the pipeline to the environment. However, this alternative would involve no treatment of the external pipeline surface that would result in a reduction of contaminant toxicity, mobility, or volume.

\section{$\underline{\text { Short-Term Effectiveness }}$}

Grouting, excavation, downsizing, and disposal of the pipelines would be a relatively straightforward processes; however, due to the extensive handling of contaminated media, there could be significant short-term risk to the health of remedial workers. However, exposure to remedial workers could be minimized and maintained below acceptable levels with the use of proper protective clothing, scheduled monitoring of area radiation and airborne particulate levels, and strict adherence to the project health and safety plan. 
LAOCB pipelines would be transported to the NTS facility located approximately $3,500 \mathrm{~km}$ $(2,200 \mathrm{mi})$ from SRS. Although transport of contaminated media from the LAOCB waste unit would increase the risk for exposure to contaminated materials, scheduling, road selection, and transport notifications should adequately reduce the risk of unwarranted exposure and accidents. The disposal process should pose minimal risk to disposal facility operators, the community, and the environment.

Alternative P-4 should pose no significant risk to the environment. Erosion control measures would be taken to minimize the potential for erosion in areas where soil would be moved. Alternative P-4 should take approximately three months to complete after mobilization to the field.

\section{Implementability}

Alternative P-4 would require conventional grouting and excavation equipment; however, extensive waste handling is required. In addition, there may public concern regarding the long distance transport of the waste. No future remedial actions would be evaluated following implementation of Alternative P-4.

\section{$\underline{\text { Cost }}$}

Costs associated with Alternative P-4 include the labor and materials needed to excavate, transport, and dispose of the LAOCB pipelines. Due to the direct handling of the pipelines, an assumed standby time is included in estimated costs to account for potential work delays that may be required to address health and safety issues. Because disposal is considered a permanent solution, there are no long-term operation and maintenance costs associated with this alternative. Costs are included for comparative purposes only and are not intended for forecasting budgetary 
expenditures. Estimated costs associated with Alternative P-4 are summarized below. Detailed costs for Alternative P-4 are provided in Appendix B.

\begin{tabular}{lr} 
Capital Costs & $\$ 4,630,000$ \\
O\&M Costs & $\$$ \\
\hline Total Costs & $\$ 4,630,000$
\end{tabular}

\subsubsection{Comparative Analysis}

Following is a comparative analysis of the four remedial alternatives being considered for remediating the LAOCB pipelines. Comparisons are summarized in Table 4-2. Remedial alternatives are evaluated against the NCP threshold (overall protection of human health, compliance with ARARs) and primary balancing criteria (long-term effectiveness and permanence; reduction of toxicity, mobility, or volume; short-term effectiveness; implementability; and cost).

\subsubsection{Overall Protection of Human Health and the Environment}

The No Action Alternative (P-1) would not be protective of human health and the environment. According to data gathered during the RFI/RI, the internal surface of the pipelines exhibit a relatively high radioactivity level (approximately $300,000 \mathrm{dpm}$ on internal surface); however radioisotopes have not been detected in the pipeline soil or in groundwater from the pipeline areas. This suggests radionuclides have not migrated from the pipeline to unit soil and groundwater. However, the No Action Alternative does not prohibit access to the pipeline areas, and the potential exists for human or wildlife intrusion and subsequent exposure to the pipelines. Furthermore, based on the shallow depth of the pipeline [within $0.91 \mathrm{~m}(3 \mathrm{ft})$ of the ground surface], its relatively high radioactivity level on the internal surface, the age ( $>30$ years) and 
material of the pipe (cast iron which could degrade over time and release radioisotopes), Alternative P-1 (No Action) would not be protective of human health or the environment.

Remaining alternatives being considered would all be protective of human health and the ecological receptors. Alternative P-2 would not result in the mitigation of potential future radionuclide release to the environment due to corrosion of the steel walls of the pipelines; however, Alternative P-2 would involve capping, which would (1) act as a barrier that would deter human access to contaminated media, (2) minimize infiltration and leaching of contaminants to unit soil and groundwater, and (3) act as shielding to reduce radiation exposure to hypothetical receptors to within acceptable levels. Alternatives P-3 and P-4 are protective of human health and the environment because they involve the excavation and disposal of the pipelines.

\subsubsection{Compliance with ARARs}

The potential chemical-specific ARARs associated with the pipelines include concentrationbased standards specified in DOE Order 5400.5. DOE Order 5400.5 is considered TBC information only. The DOE Order specifies an allowable annual effective dose to any member of the public resulting from all DOE operations of $100 \mathrm{mrem} / \mathrm{year}$. The pipelines were not sampled for specific isotopes, therefore it is unknown whether the radiation doses resulting from isotopes in the LAOCB pipelines meet the identified potential ARAR. Consequently, compliance with this potential ARAR cannot be evaluated for Alternative P-1. The remaining alternatives would comply with the potential chemical-specific ARAR through reduction of radiation dose (capping), treatment, or disposal.

No location-specific ARARs were identified under any of the alternatives. However, Alternatives P-2, P-3, and P-4 would require compliance with several action-specific ARARs. Alternative P-2 involves construction of a cap and would therefore require compliance with 
RCRA cap performance standards. Alternatives P-3 and P-4 involve construction-type activities and would require NESHAPs air modeling and permitting, an erosion control plan, and an OSHA worker health and safety plan. All alternatives could comply with the action-specific ARARs.

\subsubsection{Long-Term Effectiveness and Permanence}

Long-term effectiveness and permanence can be measured in broad terms by (1) the magnitude of residual risks associated with the waste unit, and (2) the adequacy of controls after implementation of the remedial alternative. The alternative having the highest residual risks is Alternative P-1 because the pipelines would remain in place without treatment and institutional controls would not be guaranteed under this alternative. Alternative P-2 would have less residual risk than Alternative P-1 because it would involve capping the pipeline areas which would minimize migration of contamination and would restrict human and wildlife access to the pipelines. Alternatives P-3 and P-4 would result in the least residual risk because they involve removing pipeline contamination from the area.

The adequacy of controls under Alternative P-1 cannot be ascertained since the continued maintenance institutional controls would not be guaranteed. Alternative P-2 would include the construction of a cap over the pipeline areas which would require maintenance, but would limit the radiation exposure potential, decrease the potential for migration, and limit access to the pipelines. Alternatives P-3 and P-4 involve the removal of pipeline contamination and would, therefore, not require any controls following remediation.

\subsubsection{Reduction of Toxicity, Mobility, or Volume}

Alternatives P-1, and P-2 offer no form of active treatment and, therefore, do not satisfy the NCP preference for remedial alternatives that offer a reduction in contaminant toxicity, mobility, 
or volume. However, Alternative P-2 offers capping which would reduce the mobility of contaminants by minimizing surface water infiltration, thereby reducing leaching of contaminants to unit groundwater. Alternatives P-3 and P-4 would offer active treatment through grouting that would reduce contaminant mobility.

\subsubsection{Short-Term Effectiveness}

The short-term risks to remedial workers increases with project duration. Handling (e.g., excavating, moving) and/or processing (e.g., treating) contaminated media increases the risk of remedial worker exposure to radiation effects. In addition, remedial workers are exposed to potential construction-related risks (e.g., falls, cuts, heavy equipment operation) which increase with corresponding increases in project duration; however, potential short-term risks to remedial workers should be manageable for all alternatives being considered. With strict adherence to project health and safety plans, it should be possible to maintain short-term risks of all considered alternatives within acceptable limits.

The potential risk to remedial workers would be lowest for the No Action alternative, followed by Alternative P-2, P-3, and P-4. Alternative P-2 (capping) would not involve any contact with the pipelines. Alternatives P-3 and P-4 would involve in-situ S/S and excavation and disposal of the pipelines. Alternative P-4 involves more waste handling due to downsizing and packaging of the pipeline for transport. The risk to remedial workers would be medium under Alternative P-3 and high under Alternative P-4. Alternative P-3 is estimated to take two months and Alternative P-4 three months.

All alternatives would pose negligible or low short-term risks to the community. The risks posed to the community from Alternatives P-1, P-2, and P-3, would be negligible since they would not include off-unit transport of contaminated media. Since Alternative P-4 involves transport of 
contaminated soil to the NTS facility near Mercury, Nevada, 3,500 km (2,200 mi) from the $\mathrm{LAOCB}$ waste unit, this alternative involves more risk than the other alternatives.

\subsubsection{Implementability}

Alternative P-1 (No Action) would be the most implementable alternative being considered since it would not involve any type of construction or remedial actions beyond existing institutional controls; however, the No Action Alternative could potentially arouse public concern since it does not involve treatment or removal of the contamination. Alternative P-2 (Capping) would involve the construction of a cap, but should be relatively easy to implement. Alternative P-2 should not elicit major public concerns since a cap would provide a physical barrier between receptors and the pipelines. Alternative P-3 (In-Situ S/S and disposal in the LAOCB) and P-4 (In-Situ S/S and disposal at NTS) could be readily implementable. S/S is a common applied technology for remediating low-level wastes and should not elicit public concerns. However, there may be potential public concern regarding the off-site transportation of low-level waste under Alternative P-4.

\subsubsection{Cost}

Total estimated present worth costs range between $\$ 730,000$ for Alternative P-2 (Capping) to $\$ 4,630,000$ for Alternative P-4 (In-situ S/S, excavation, and disposal at the NTS). The costs associated with alternatives are provided in Table 4-2. The cost of Alternative P-1, No Action, would be included under the No Action alternative for the LAOCB soils (S-1). Alternative P-2 $(\$ 730,000)$ include capping only. Alternative P-3 $(\$ 990,000)$ involves the grouting, excavation,

and disposal of the pipelines in the LAOCB. Alternative P-4 $(\$ 4,630,000)$ would involve grouting, excavation, and disposal of the LAOCB pipelines at the NTS. 
Alternatives P-1 and P-2 would require a remedy review every five years for 30 years because they do not result in unrestricted use of the pipeline areas; however, the cost for remedy review would be included with that of the LAOCB soils, depending on the remedy selected for the LAOCB. Alternative P-2 includes the operation and maintenance costs of a cap. Cost estimates are provided in Appendix B for each of the alternatives. 
THIS PAGE INTENTIONALLY LEFT BLANK 
CHAPTER 4 FIGURES 
THIS PAGE INTENTIONALLY LEFT BLANK 


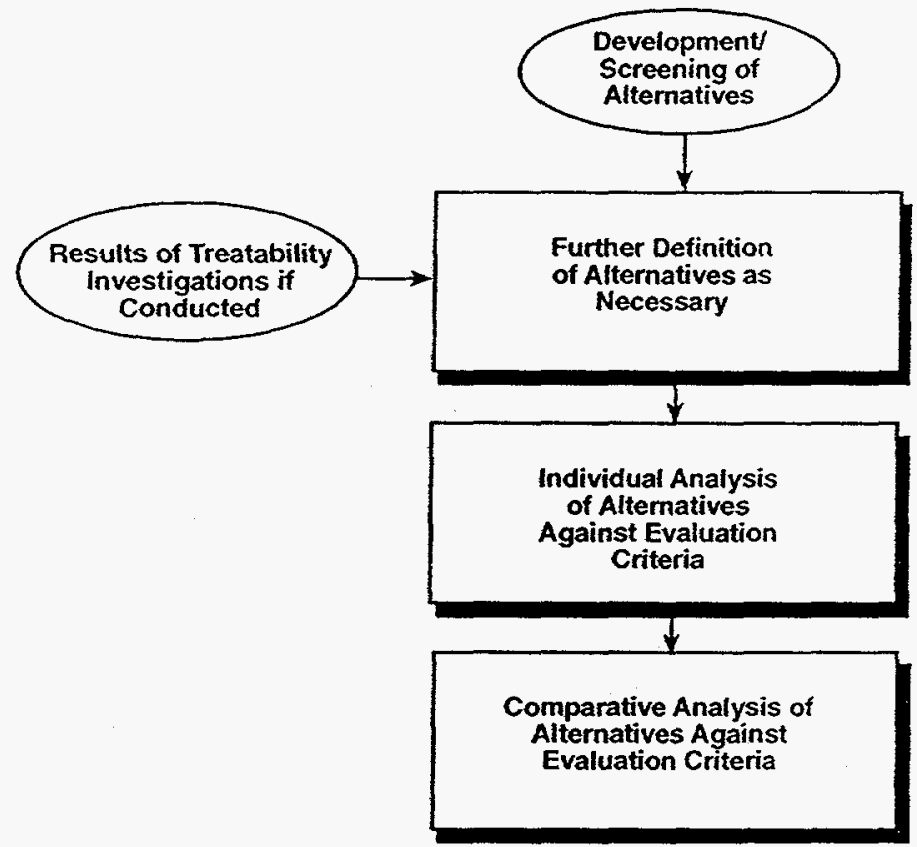

Source: EPA, 1988a

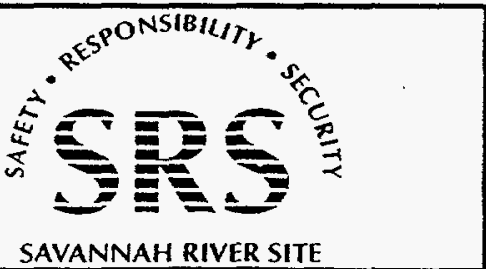

FIGURE 4-1

COMPONENTS OF THE DETAILED

ANALYSIS OF ALTERNATIVES

L-Area Oil and Chernical Basin

Savannah River Site 


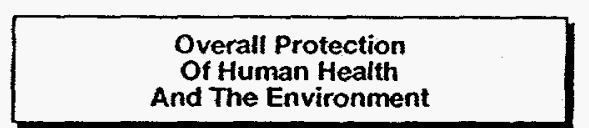

- How Alternative Provides Human Health and Environmental Protection

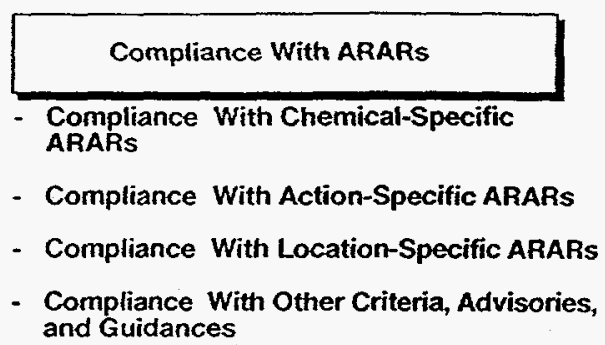
and Guidances

\begin{tabular}{|c|c|}
\hline $\begin{array}{l}\text { Long-Term } \\
\text { Effectiveness } \\
\text { And Permanence }\end{array}$ & $\begin{array}{l}\text { Reduction Of Toxicity } \\
\text { Mobility, And Volume } \\
\text { Through Treatment }\end{array}$ \\
\hline $\begin{array}{l}\text { - Magnitude of } \\
\text { Residual Risk }\end{array}$ & $\begin{array}{l}\text { Treatment Process Used } \\
\text { And Materials Treated }\end{array}$ \\
\hline \multirow[t]{4}{*}{$\begin{array}{l}\text { - Adequacy And } \\
\text { Reliability Of } \\
\text { Controls }\end{array}$} & $\begin{array}{l}\text { Amount Of Hazardous } \\
\text { Materials Destroyed Or } \\
\text { Treated }\end{array}$ \\
\hline & $\begin{array}{l}\text { Degree Of Expected } \\
\text { Reductions In Toxicity, } \\
\text { Mobility, And Volume }\end{array}$ \\
\hline & $\begin{array}{l}\text { - Degree To Which } \\
\text { Treatment Is Irreversible }\end{array}$ \\
\hline & $\begin{array}{l}\text { - Type And Quality of } \\
\text { Residuals Remaining After } \\
\text { Treatment }\end{array}$ \\
\hline
\end{tabular}

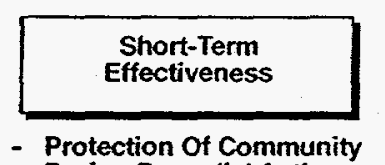

- Protection of Community During Remedial Actions

- Protection Of Workers During Remedial Actions

- Environmental Impacts

- Time Until Remedial Action Objectives Are Achieved

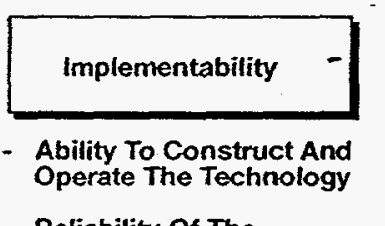

- Reliability of The Technology

- Ease Of Undertaking Additional Remedial Actions, If Necessary

- Ability To Monitor Effectiveness of Remedy

- Ability To Obtain Approvals From Other Agencies

- Coordination With Other Agencies

- Availability of Offsite Treatment, Storage, And Disposal Services And Capacity

- Availability Of Necessary Equipment And Specialists

- Availability Of Prospective Technologies

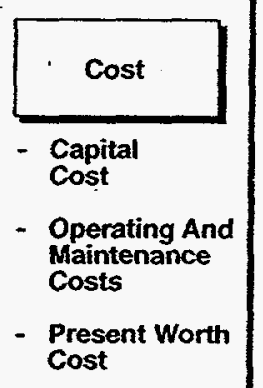

Present Worth
Cost cost 


\section{THIS PAGE INTENTIONALLY LEFT BLANK}




\section{FOUR GENERAL STANDARDS FOR CORRECTIVE MEASURES}

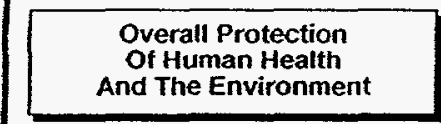

- How Alternative Provides Human Health and Environmental Protection

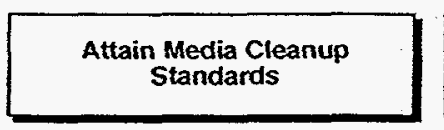

- Ability Of Alternatives To Acheive The Media Cleanup Standards Prescribed In The Permit Modification Or Enforcement Order
Control The Sources of Releases

- How Altematives Reduce Or Eliminate To The Maximum Extent Possible Further Releases
Comply with Standards For Management Of Wastes

- How Alternatives Assure That Management Of Wastes During Corrective Measures is Conducted In A Protective Manner

FIVE SELECTION DECISION FACTORS

\begin{tabular}{c|}
\hline Long-Term \\
Effectiveness \\
And Permanence
\end{tabular}

- Magnitude of Residuat Risk

- Adequacy And Retiability of Controls

\begin{tabular}{l}
$\begin{array}{l}\text { Reduction Of Toxicity } \\
\text { Mobility, And Volume } \\
\text { Through Treatment }\end{array}$ \\
\hline Treatment Process Used \\
And Materials Treated \\
- Amount Of Hazardous \\
Materials Destroyed Or \\
Treated \\
- Degree Of Expected \\
Reductions In Toxicity, \\
Mobility, And Volume \\
- Degree To Which \\
Treatment Is Imeversible \\
Type And Quality Of \\
Residuais Remaining After \\
Treatment
\end{tabular}

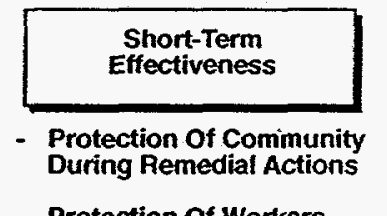

- Protection of Workers During Remedial Actions

- Environmental Impacts

Time Until Remedial Achieved Treatment Action Objectives Are

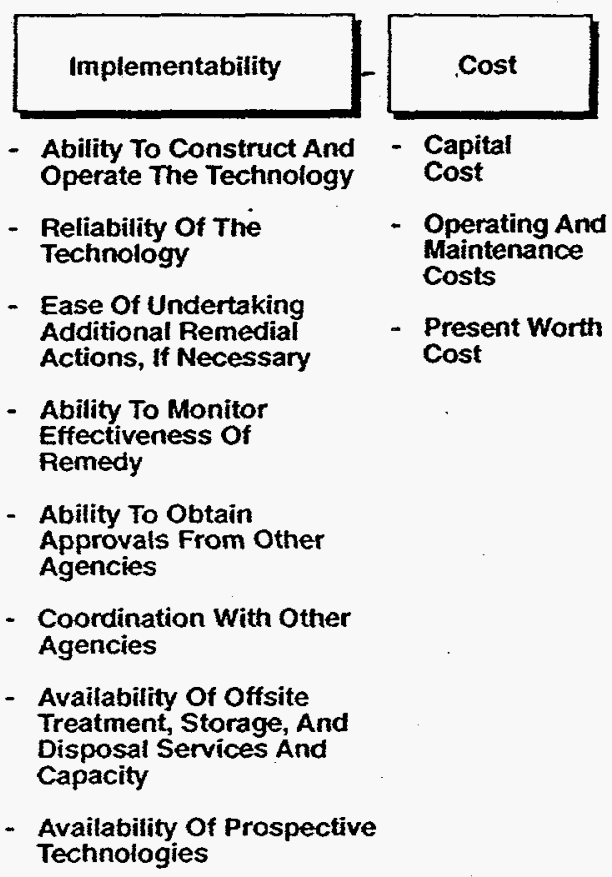

Source: EPA 1988 a

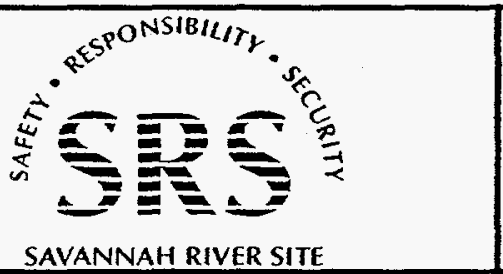

FIGURE 4-3

COMPONENTS OF THE NINE CMS CRITERIA

1-Area Oil and Chemical Basin Savannah River Site 
PHASE I FOCUSED CMS/FS REPORT

L-AREA OIL AND CHEMICAL BASIN
WSRC-RP-96-106, REV. 1.1

FEBRUARY 1997

\section{THIS PAGE INTENTIONALLY LEFT BLANK}


PHASE I FOCUSED CMS/FS REPORT

L-AREA OIL AND CHEMICAL BASIN
WSRC-RP-96-106, REV. 1.1

FEBRUARY 1997

\section{CHAPTER 4}

TABLES 


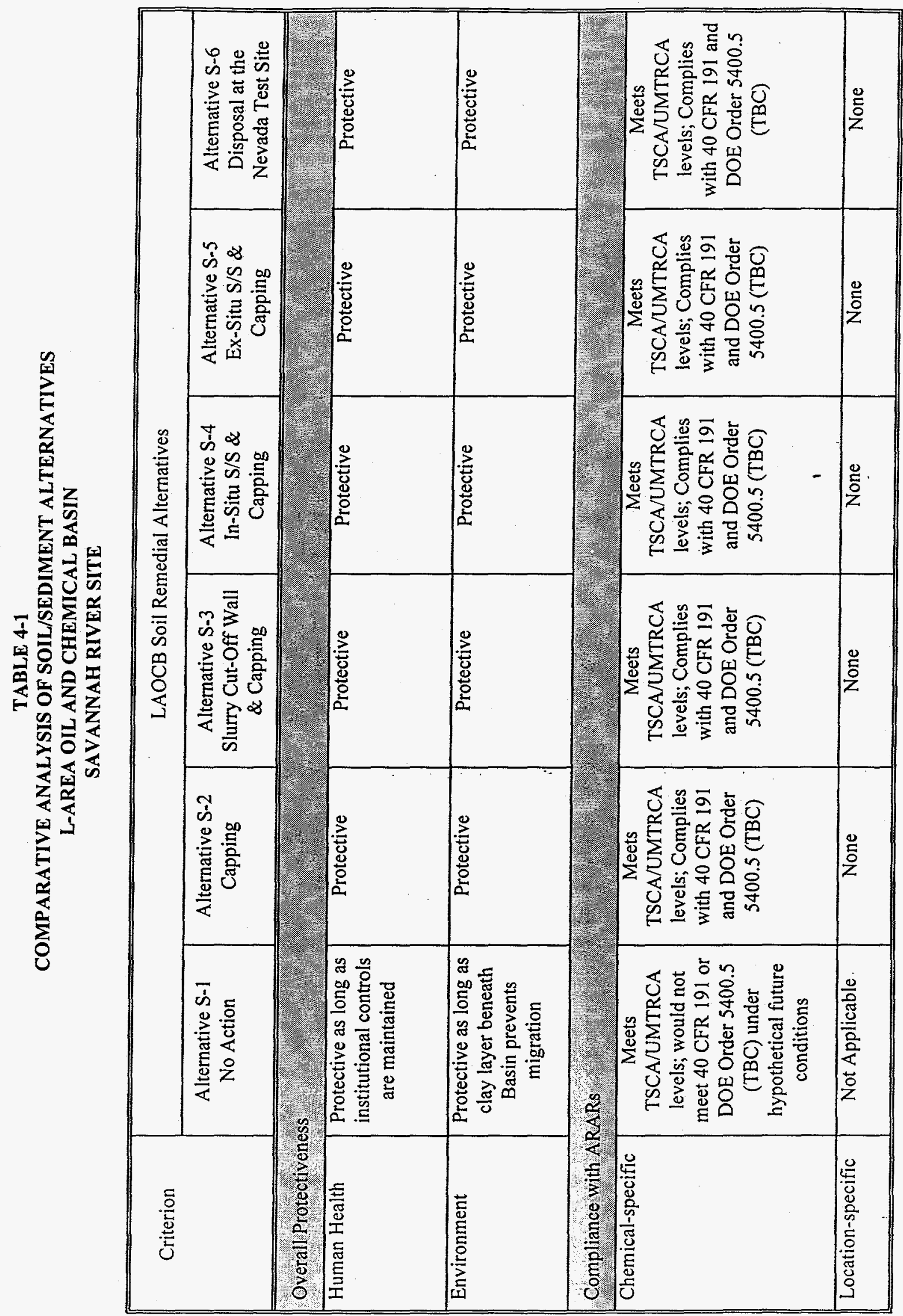


TABLE 4-1 (CONTINUED)

COMPARATIVE ANALYSIS OF SOIL/SEDIMENT ALTERNATIVES

L-AREA OIL AND CHEMICAL BASIN

SAVANNAH RIVER SITE

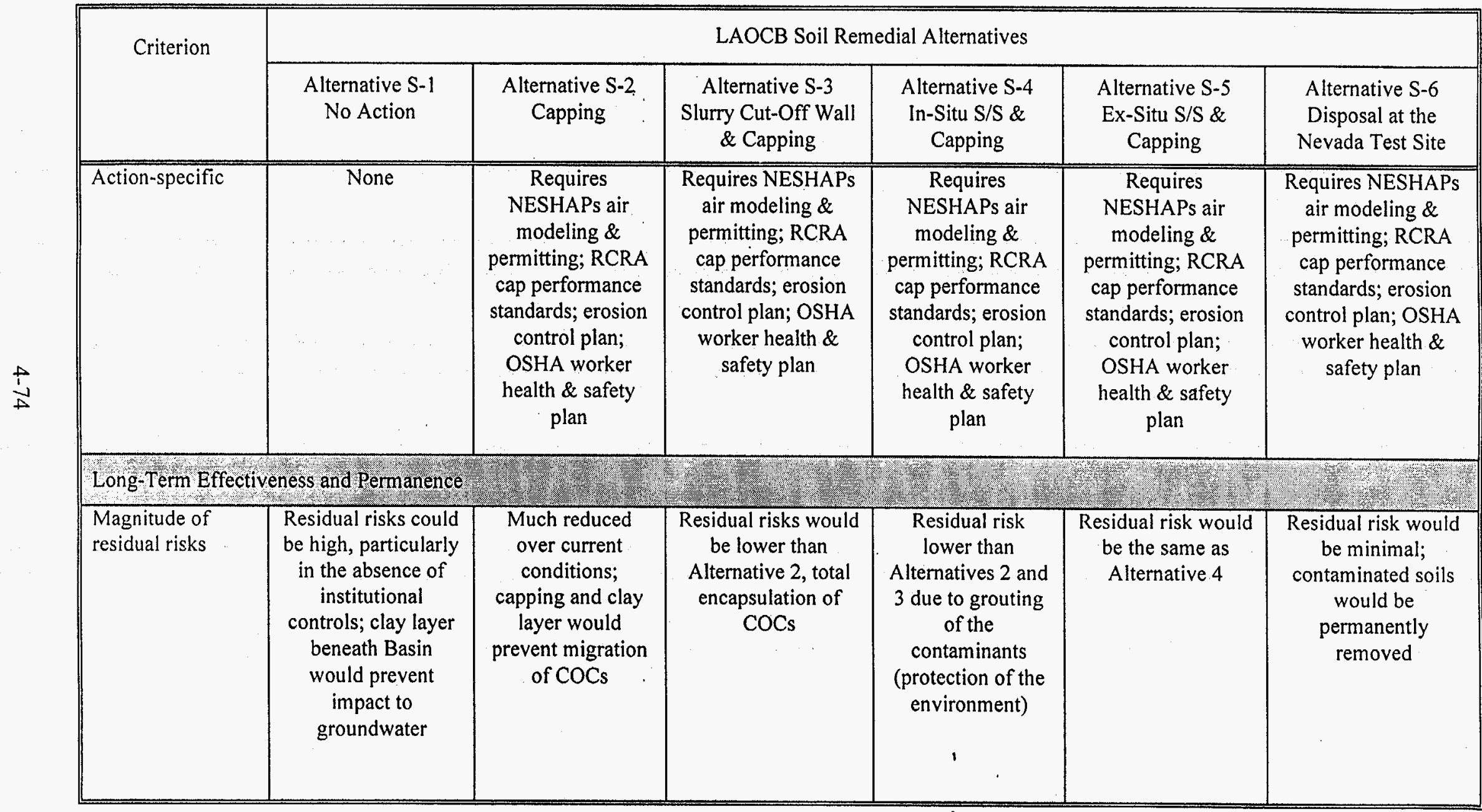


TABLE 4-1 (CONTINUED)

COMPARATIVE ANALYSIS OF SOIL/SEDIMENT ALTERNATIVES

L-AREA OIL AND CHEMICAL BASIN

SAVANNAH RIVER SITE

\begin{tabular}{|c|c|c|c|c|c|c|}
\hline \multirow{2}{*}{ Criterion } & \multicolumn{6}{|c|}{ LAOCB Soil Remedial Alternatives } \\
\hline & $\begin{array}{l}\text { Alternative S-1 } \\
\text { No Action }\end{array}$ & $\begin{array}{l}\text { Alternative S-2 } \\
\text { Capping }\end{array}$ & $\begin{array}{l}\text { Alternative S-3 } \\
\text { Slurry Cut-Off Wall } \\
\text { \& Capping }\end{array}$ & $\begin{array}{l}\text { Alternative S-4 } \\
\text { In-Situ S/S \& } \\
\text { Capping }\end{array}$ & $\begin{array}{l}\text { Alternative S-5 } \\
\text { Ex-Situ S/S \& } \\
\text { Capping }\end{array}$ & $\begin{array}{l}\text { Alternative S-6 } \\
\text { Disposal at the } \\
\text { Nevada Test Site }\end{array}$ \\
\hline $\begin{array}{l}\text { Adequacy of } \\
\text { controls }\end{array}$ & $\begin{array}{l}\text { Existing } \\
\text { institutional controls } \\
\text { are effective for the } \\
\text { protection of human } \\
\text { health, but cannot } \\
\text { be guaranteed; } \\
\text { adequacy of the clay } \\
\text { layer has proven } \\
\text { effective, but would } \\
\text { not be verified }\end{array}$ & $\begin{array}{c}\text { Existing and } \\
\text { supplemental } \\
\text { institutional } \\
\text { controls would be } \\
\text { effective; cap and } \\
\text { the clay layer } \\
\text { beneath the Basin } \\
\text { would prevent } \\
\text { migration of } \\
\text { COCs }\end{array}$ & $\begin{array}{c}\text { Existing and } \\
\text { supplemental } \\
\text { institutional controls } \\
\text { would be effective; } \\
\text { slurry wall, cap and } \\
\text { the clay layer } \\
\text { beneath the Basin } \\
\text { would prevent } \\
\text { migration of COCs }\end{array}$ & $\begin{array}{l}\text { Existing and } \\
\text { supplemental } \\
\text { institutional } \\
\text { controls would be } \\
\text { effective and } \\
\text { grouting of the } \\
\text { contaminated soils } \\
\text { would further } \\
\text { limit risk to the } \\
\text { environment }\end{array}$ & $\begin{array}{l}\text { Existing and } \\
\text { supplemental } \\
\text { institutional } \\
\text { controls would be } \\
\text { effective and } \\
\text { grouting of the } \\
\text { contaminated soils } \\
\text { would further limit } \\
\text { risk to the } \\
\text { environment }\end{array}$ & $\begin{array}{l}\text { No controls required; } \\
\text { could be released for } \\
\text { unrestricted land use }\end{array}$ \\
\hline Treatment type & No active treatment & $\begin{array}{l}\text { No active } \\
\text { treatment }\end{array}$ & No active treatment & $\begin{array}{c}\text { Stabilization/ } \\
\text { solidification of } \\
\text { the contaminated } \\
\text { soil }\end{array}$ & $\begin{array}{c}\text { Stabilization/ } \\
\text { solidification of the } \\
\text { contaminated soil }\end{array}$ & None \\
\hline
\end{tabular}


TABLE 4-1 (CONTINUED)

COMPARATIVE ANALYSIS OF SOIL/SEDIMENT ALTERNATIVES

L-AREA OIL AND CHEMICAL BASIN

SAVANNAH RIVER SITE

\begin{tabular}{|c|c|c|c|c|c|c|}
\hline Criterion & \multicolumn{6}{|c|}{ LAOCB Soil Remedial Alternatives } \\
\hline $\begin{array}{l}\text { Reduction of } \\
\text { toxicity, mobility } \\
\text { or volume }\end{array}$ & $\begin{array}{l}\text { None through } \\
\text { treatment }\end{array}$ & $\begin{array}{l}\text { Capping and the } \\
\text { clay layer beneath } \\
\text { the Basin would } \\
\text { effectively reduce } \\
\text { contaminant } \\
\text { mobility as long as } \\
\text { cap integrity is } \\
\text { maintained; not a } \\
\text { permanent } \\
\text { reduction in } \\
\text { contaminant } \\
\text { mobility }\end{array}$ & $\begin{array}{l}\text { Slurry wall, capping } \\
\text { and the clay layer } \\
\text { beneath the Basin } \\
\text { would effectively } \\
\text { reduce contaminant } \\
\text { mobility as long as } \\
\text { cap integrity is } \\
\text { maintained; not a } \\
\text { permanent reduction } \\
\text { in contaminant } \\
\text { mobility }\end{array}$ & $\begin{array}{l}\text { Permanently } \\
\text { reduce } \\
\text { contaminant } \\
\text { mobility in the } \\
\text { soils }\end{array}$ & $\begin{array}{l}\text { Permanently reduce } \\
\text { contaminant } \\
\text { mobility in the soils }\end{array}$ & $\begin{array}{l}\text { Contaminated soils } \\
\text { removed, but } \\
\text { relocated }\end{array}$ \\
\hline $\begin{array}{l}\text { Risk to } \\
\text { community }\end{array}$ & Negligible & Minimal & Minimal & Minimal & Minimal & $\begin{array}{l}\text { Medium; would } \\
\text { involve transport of } \\
\text { soils to the NTS }\end{array}$ \\
\hline $\begin{array}{l}\text { Construction } \\
\text { schedule }\end{array}$ & $\begin{array}{c}\text { Immediately } \\
\text { implementable }\end{array}$ & 3 months & 6 months & 12 months & 15 months & 3 months \\
\hline
\end{tabular}


TABLE 4-1 (CONTINUED)

COMPARATIVE ANALYSIS OF SOIL/SEDIMENT ALTERNATIVES

L-AREA OIL AND CHEMICAL BASIN

SAVANNAH RIVER SITE

\begin{tabular}{|c|c|c|c|c|c|c|}
\hline \multirow{2}{*}{ Criterion } & \multicolumn{6}{|c|}{ LAOCB Soil Remedial Alternatives } \\
\hline & $\begin{array}{c}\text { Alternative S-1 } \\
\text { No Action }\end{array}$ & $\begin{array}{c}\text { Alternative S-2 } \\
\text { Capping }\end{array}$ & $\begin{array}{l}\text { Alternative S-3 } \\
\text { Slurry Cut-Off Wall } \\
\text { \& Capping }\end{array}$ & $\begin{array}{l}\text { Alternative S-4 } \\
\text { In-Situ S/S \& } \\
\text { Capping }\end{array}$ & $\begin{array}{l}\text { Alternative S-5 } \\
\text { Ex-Situ S/S \& } \\
\text { Capping }\end{array}$ & $\begin{array}{l}\text { Alternative S-6 } \\
\text { Disposal at the } \\
\text { Nevada Test Site }\end{array}$ \\
\hline Potential concerns & $\begin{array}{l}\text { Potential for public } \\
\text { concern if no action } \\
\text { is implemented }\end{array}$ & $\begin{array}{l}\text { Potential for } \\
\text { public concern } \\
\text { since no treatment } \\
\text { is performed }\end{array}$ & $\begin{array}{l}\text { Potential for public } \\
\text { concern since no } \\
\text { treatment is } \\
\text { performed }\end{array}$ & None & $\begin{array}{l}\text { Medium; would } \\
\text { require pre- } \\
\text { excavation } \\
\text { treatment for waste } \\
\text { handling purposes }\end{array}$ & $\begin{array}{l}\text { High; would involve } \\
\text { transport of soils } \\
\text { outside SRS } \\
\text { boundaries; would } \\
\text { require pre- \& post- } \\
\text { excavation treatment } \\
\text { for waste handling \& } \\
\text { packaging purposes }\end{array}$ \\
\hline $\begin{array}{l}\text { Relative } \\
\text { implementability }\end{array}$ & $\begin{array}{c}\text { Readily } \\
\text { implementable }\end{array}$ & $\begin{array}{l}\text { Readily } \\
\text { implementable, } \\
\text { but would require } \\
\text { much more effort } \\
\text { than No Action }\end{array}$ & $\begin{array}{c}\text { Readily } \\
\text { implementable; } \\
\text { would require more } \\
\text { effort than capping } \\
\text { alone (Alt 2) }\end{array}$ & $\begin{array}{c}\text { Readily } \\
\text { implementable; } \\
\text { would require } \\
\text { more effort than } \\
\text { capping alone } \\
\text { (Alt 2) }\end{array}$ & $\begin{array}{l}\text { Implementable; } \\
\text { however, waste } \\
\text { handling may cause } \\
\text { down time during } \\
\text { remediation; also } \\
\text { requires pre- } \\
\text { excavation } \\
\text { treatment }\end{array}$ & $\begin{array}{l}\text { Implementable; } \\
\text { however, waste } \\
\text { handling may cause } \\
\text { down time during } \\
\text { remediation; also } \\
\text { requires pre- \& post- } \\
\text { excavation treatment }\end{array}$ \\
\hline
\end{tabular}


TABLE 4-1 (CONTINUED)

COMPARATIVE ANALYSIS OF SOIL/SEDIMENT ALTERNATIVES

L-AREA OIL AND CHEMICAL BASIN

SAVANNAH RIVER SITE

\begin{tabular}{|c|c|c|c|c|c|c|}
\hline \multirow{2}{*}{ Criterion } & \multicolumn{6}{|c|}{ LAOCB Soil Remedial Alternatives } \\
\hline & $\begin{array}{c}\text { Alternative S-1 } \\
\text { No Action }\end{array}$ & $\begin{array}{c}\text { Alternative S-2 } \\
\text { Capping }\end{array}$ & $\begin{array}{l}\text { Alternative S-3 } \\
\text { Slurry Cut-Off Wall } \\
\& \text { Capping }\end{array}$ & $\begin{array}{l}\text { Alternative S-4 } \\
\text { In-Situ S/S \& } \\
\text { Capping }\end{array}$ & $\begin{array}{c}\text { Alternative S-5 } \\
\text { Ex-Situ S/S \& } \\
\text { Capping }\end{array}$ & $\begin{array}{l}\text { Alternative S-6 } \\
\text { Disposal at the } \\
\text { Nevada Test Site }\end{array}$ \\
\hline $\begin{array}{l}\text { Basis for O\&M } \\
\text { costs }\end{array}$ & 30 years & 30 years & 30 years & 30 years & 30 years & Not applicable \\
\hline $\begin{array}{l}\text { Present worth } \\
\text { capital costs }\end{array}$ & $\$ 0$ & $\$ 1,000,000$ & $\$ 3,000,000$ & $\$ 3,150,000$ & $\$ 3,940,000$ & $\$ 9,100,000$ \\
\hline $\begin{array}{l}\text { Present worth } \\
\text { O\&M costs }\end{array}$ & $\$ 280,000$ & $\$ 430,000$ & $\$ 430,000$ & $\$ 430,000$ & $\$ 430,000$ & $\$ 0$ \\
\hline $\begin{array}{l}\text { Total present } \\
\text { worth costs }\end{array}$ & $\$ 280,000$ & $\$ 1,430,000$ & $\$ 3,430,000$ & $\$ 3,580,000$ & $\$ 4,370,000$ & $\$ 9,100,000$ \\
\hline
\end{tabular}

*Costs are developed for comparison purposes only and are not intended to forecast actual expenditures.

$\mathrm{S} / \mathrm{S}=$ Stabilization/Solidification 
TABLE 4-2

COMPARATIVE ANALYSIS OF PIPELINE ALTERNATIVES

L-AREA OIL AND CHEMICAL BASIN

SAVANNAH RIVER SITE

\begin{tabular}{|c|c|c|c|c|}
\hline Criterion & \multicolumn{4}{|c|}{ LAOCB Pipelines Remedial Alternatives } \\
\hline \multicolumn{5}{|l|}{ Overall Protectiveness. } \\
\hline Human Health & Not Protective & Protective & Protective & Protective \\
\hline Chemical-specific & $\begin{array}{l}\text { Compliance with DOE Order } \\
5400.5 \text { cannot be determined } \\
\text { until further investigation is } \\
\text { conducted }\end{array}$ & $\begin{array}{l}\text { Would comply with DOE } \\
\text { Order } 5400.5\end{array}$ & $\begin{array}{l}\text { Would comply with DOE } \\
\text { Order } 5400.5\end{array}$ & $\begin{array}{l}\text { Would comply with DOE } \\
\text { Order } 5400.5\end{array}$ \\
\hline Location-specific & None & None & None & None \\
\hline \multicolumn{5}{|c|}{ Long-Term Effectiveness and Performance. } \\
\hline Magnitude of residual risks & $\begin{array}{l}\text { Risk would include intrusion } \\
\text { into the pipeline, and future } \\
\text { release of contaminants due } \\
\text { to degradation of the } \\
\text { pipelines integrity }\end{array}$ & $\begin{array}{l}\text { Residual risks would be } \\
\text { lower than Alternative } 1 \\
\text { capping would decreases } \\
\text { access, migration and } \\
\text { external exposure potential }\end{array}$ & $\begin{array}{l}\text { Residual risk would be } \\
\text { minimal; the pipeline would } \\
\text { be grouted in place and } \\
\text { permanently removed; } \\
\text { contaminants would be } \\
\text { transferred to the basin to be } \\
\text { stabilized further }\end{array}$ & $\begin{array}{l}\text { Residual risk would be } \\
\text { minimal; the pipeline } \\
\text { would be permanently } \\
\text { removed }\end{array}$ \\
\hline
\end{tabular}


TABLE 4-2 (CONTINUED)

COMPARATIVE ANALYSIS OF PIPELINE ALTERNATIVES

L-AREA OIL AND CHEMICAL BASIN

SAVANNAH RIVER SITE

\begin{tabular}{|c|c|c|c|c|}
\hline \multirow[t]{2}{*}{ Criterion } & \multicolumn{4}{|c|}{ LAOCB Pipelines Remedial Alternatives } \\
\hline & Alternative P-1 No Action & Alternative P-2 Capping & $\begin{array}{c}\text { Alternative P-3 In-situ S/S } \\
\text { Excavation \& Disposal in the } \\
\text { LAOCB }\end{array}$ & $\begin{array}{c}\text { Alternative P-4 In-situ S/S } \\
\text { Excavation and Disposal } \\
\text { at the Nevada Test Site }\end{array}$ \\
\hline Adequacy of controls & $\begin{array}{l}\text { Existing pipeline appears to } \\
\text { prevent migration of } \\
\text { radionuclides, but migration } \\
\text { or protection against human } \\
\text { intrusion cannot be } \\
\text { guaranteed }\end{array}$ & $\begin{array}{l}\text { Capping would further } \\
\text { prevent migration of } \\
\text { contaminants by limiting } \\
\text { infiltration }\end{array}$ & $\begin{array}{l}\text { No controls required; could } \\
\text { be released for unrestricted } \\
\text { land use }\end{array}$ & $\begin{array}{l}\text { No controls required; } \\
\text { could be released for } \\
\text { unrestricted land use }\end{array}$ \\
\hline \multicolumn{5}{|c|}{ Reduction of Toxicity, Mobility or Volume } \\
\hline Treatment type & No active treatment & No active treatment & In-Situ S/S & In-Situ S/S \\
\hline $\begin{array}{l}\text { Reduction of toxicity, } \\
\text { mobility or volume }\end{array}$ & None through treatment & $\begin{array}{l}\text { None through treatment, but } \\
\text { capping would decrease the } \\
\text { mobility of contaminants }\end{array}$ & $\begin{array}{l}\text { Grouting the pipeline would } \\
\text { reduce mobility }\end{array}$ & $\begin{array}{l}\text { Grouting the pipeline } \\
\text { would reduce mobility }\end{array}$ \\
\hline \multicolumn{5}{|l|}{ Shott-Term Effectiveness } \\
\hline Risk to remedial workers & $\begin{array}{l}\text { None; would involve no } \\
\text { handling of pipelines }\end{array}$ & $\begin{array}{l}\text { Minimal; would involve no } \\
\text { contact with pipelines }\end{array}$ & $\begin{array}{l}\text { Medium; excavation would } \\
\text { require contact with the } \\
\text { pipelines, however residual } \\
\text { contaminants would be } \\
\text { immobilized before handling }\end{array}$ & $\begin{array}{l}\text { High; excavation and } \\
\text { loading would require } \\
\text { intimate contact with the } \\
\text { pipelines, however, } \\
\text { residual contaminants } \\
\text { would be immobilized } \\
\text { before handling }\end{array}$ \\
\hline Risk to community & Negligible & Negligible & Minimal & $\begin{array}{l}\text { Medium; would involve } \\
\text { transport of contaminated } \\
\text { media to the NTS }\end{array}$ \\
\hline Construction schedule & Immediately implementable & 2 months & 2 months & 3 months \\
\hline
\end{tabular}


TABLE 4-2 (CONTINUED)

COMPARATIVE ANALYSIS OF PIPELINE ALTERNATIVES

L-AREA OIL AND CHEMICAL BASIN

SAVANNAH RIVER SITE

\begin{tabular}{|c|c|c|c|c|}
\hline \multirow[t]{2}{*}{ Criterion } & \multicolumn{4}{|c|}{ LAOCB Pipelines Remedial Alternatives } \\
\hline & Alternative P-1 No Action & Alternative P-2 Capping & $\begin{array}{c}\text { Alternative P-3 In-situ S/S } \\
\text { Excavation \& Disposal in the } \\
\text { LAOCB }\end{array}$ & $\begin{array}{l}\text { Alternative P-4 In-situ S/S } \\
\text { Excavation and Disposal } \\
\text { at the Nevada Test Site }\end{array}$ \\
\hline \multicolumn{5}{|c|}{ Implementability } \\
\hline Potential concerns & May prompt public concern & None & $\begin{array}{l}\text { Medium; would involve } \\
\text { grouting, excavation, } \\
\text { pipeline downsizing, and } \\
\text { exposure to pipelines }\end{array}$ & $\begin{array}{l}\text { High; would involve } \\
\text { grouting excavation, } \\
\text { pipeline downsizing, } \\
\text { loading, and transport of } \\
\text { pipelines long distance }\end{array}$ \\
\hline Relative implementability & Readily implementable & $\begin{array}{l}\text { Readily implementable; } \\
\text { capping would require } \\
\text { significantly more effort than } \\
\text { Alternatives } 1\end{array}$ & $\begin{array}{l}\text { Implementable; however, } \\
\text { would require more effort } \\
\text { than Alternative } 2 \text { due to } \\
\text { potential for worker } \\
\text { exposure }\end{array}$ & $\begin{array}{c}\text { Implementable; however, } \\
\text { would require more effort } \\
\text { than Alternative } 3 \text { due to } \\
\text { transport }\end{array}$ \\
\hline Present worth O\&M costs & See Note 2 below & $\$ 170,000$ & $\$ 0$ & $\$ 0$ \\
\hline Total present worth costs & See Note 2 below & $\$ 730,000$ & $\$ 990,000$ & $\$ 4,630,000$ \\
\hline
\end{tabular}

1. Costs are developed for comparison purposes only and are not intended to forecast actual expenditures.

2. The cost of remedy reviews would be included in those for the LAOCB OU, depending on the remedy selected for the LAOCB.

$\mathrm{S} / \mathrm{S}=$ Stabilization/Solidification 


\section{THIS PAGE INTENTIONALLY LEFT BLANK}




\section{REFERENCES}

Benedict, M., T.H. Pigford, and H.W. Levi, 1981. Nuclear Chemical Engineering, McGraw-Hill, Inc., New York, NY.

Browne, E. and R.B. Firestone, 1986. Table of Radioactive Isotopes. Wiley-Interscience, New York.

Chapman, C.C., Robinson, R.A., 1993. Terra-Vit: A Low Cost Vitrification Technology For Site Cleanup; USDOE "ER'93-Meeting The Challenge Environmental Remediation Conference" (Proceedings Volume 2); October 24-28, 1993, Radisson Riverfront Hotel, Augusta, GA.

Conner, J.R., 1994. Hazardous Waste Soil Remediation-Theory and Application of Innovative Technolgies (edited by Wilson, D.J. and Clarke, A.N.), Marcel Dekker, Inc., New York, NY.

DOE (U.S. Department of Energy), 1985. Safety Requirements for the Packaging and Transportation of Hazardous Material, DOE Order 5480.3, U.S. Department of Energy, Washington, DC.

DOE (U.S. Department of Energy), 1988. Radioactive Waste Management, DOE Order 5820.2A, U.S. Department of Energy, Washington, DC.

DOE (U.S. Department of Energy), 1990. Radiation Protection of the Public and the Environment, DOE Order 5400.5, U.S. Department of Energy, Washington, DC.

DOE (U.S. Department of Energy), 1993. Remedial Investigation/Feasibility Study (RI/FS) Process, Elements, and Techniques Guidance, DOE/EH-940007658.

DOE (U.S. Department of Energy), 1994a. Public Involvement, A Plan of the Savannah River Site. Savannah River Operations Office, Aiken, SC.

DOE (U.S. Department of Energy), January 1994b. Implementation Plan-Environmental Restoration and Waste Management Programmatic Environmental Impact Statement; Prepared by the U.S. Department of Energy Environmental Restoration and Waste Management Program; Washington, DC.

DOE (U.S. Department of Energy), 1995. Closing the Circle on the Splitting of the Atom, Office of Environmental Management, U.S. Department of Energy, Washington, DC. 
England J.L., et al., August 1991. Perspective Of Hazardous Waste And Mixed Waste Treatment Technology At The Savannah River Site; Mixed Waste-Proceedings of the First International Symposium, August 26-29, 1991, Baltimore, MD.

EPA (U.S. Environmental Protection Agency), 1985. Handbook - Remedial Action At Waste Disposal Sites (Revised), (EPA/625/6-85/006); Office of Research and Development Hazardous Waste Engineering Research Laboratory, Cincinnati, $\mathrm{OH}$.

EPA (U.S. Environmental Protection Agency), 1988a. Guidance for Conducting Remedial Investigations and Feasibility Studies Under CERCLA, Interim Final, EPA/540/G-89004, U.S. Environmental Protection Agency, Washington, DC.

EPA (U.S. Environmental Protection Agency), 1988b. RCRA Corrective Action Plan, EPA/530SW-88-028, Interim Final. U.S. Environmental Protection Agency, Washington, DC.

EPA (U.S. Environmental Protection Agency), 1988c. CERCLA Compliance with Other Laws Manual, Draft Guidance, OSWER Directive 9234.1-01, U.S. Environmental Protection Agency, Washington, DC.

EPA (U.S. Environmental Protection Agency), 1988d. Technological Approaches to the Cleanup of Radiologically Contaminated Superfund Sites, (EPA/540/2-88/002); Office of Research and Development, Washington, DC.

EPA (U.S. Environmental Protection Agency), 1992. Vitrification Technologies for Treatment of Hazardous and Radioactive Waste, (EPA/625/R-92/002); Office of Research and Development, Washington, DC.

EPA (U.S. Environmental Protection Agency), 1993a. Remediation Technologies Screening Matrix and Reference Guide, EPA 542-B-93-005, U.S. Environmental Protection. Agency, Washington, DC.

EPA (U.S. Environmental Protection Agency), 1993b. Handbook-Approaches for the Remediation of Federal Facility Sites Contaminated with Explosive or Radioactive Wastes, (EPA/625/R-93/013); Office of Research and Development, Washington, DC.

EPA (U.S. Environmental Protection Agency), 1994. Superfund Innovative Technology Evaluation Program: Technology Profiles, Seventh Edition, EPA/540/R-94/526, U.S. Environmental Protection Agency, Washington, DC.

Envirocare, 1994. Utah Department of Environmental Quality Division of Radiation Control, Radioactive Material License No. UT2300249; Utah Bureau of Solid and Hazardous Waste Permit, EPA I.D. No. UTD982598898. 
Fenimore, J.W., J.H, Horton, Jr., K.B. Broom, and N.M. Park, 1988. Radionuclides in the Ground at the Savannah River Plant, DPST-74-319, Rev. 1, E.I. du Pont de Nemours and Company, Savannah River Laboratory, Aiken, SC.

Fay, W.M. and J.B. Pickett, 1987. Documentation of 1982 Soil Analyses to Determine the Natural Background Radioactivity in SRP Surface Soils, Technical Memorandum to D.E. Gordon, DPST-87-260, E.I. du Pont de Nemours and Company, Savannah River Plant, Aiken, SC.

HSDB (Hazardous Substances Data Bank), 1994. National Library of Medicine (NLM) On-Line Toxicological Network (TOXNET), Bethesda, MD.

Jarvis, W.L., 1993. Threatened, Endangered, and Sensitive Species Listing, L-Area Basins, U.S. Department of Agriculture, Savannah River Forest Station, Savannah River Site, Aiken, SC.

Kittel, H.J., ed., 1989. Radioactive Waste Management Handbook Volume 1: Near-Surface Land Disposal, Harwood Academic Publishers, London, England.

LaGrega, M.D., P.L. Buckingham, and J.C. Evans, 1994. Hazardous Waste Management, McGraw-Hill, Inc., New York, NY.

Looney, B.B., C.A. Eddy, M. Rammed, J. Pickett, V. Rogers, M.T. Scotta nd PA. Shirley, 1990. Geochemical and Physical Properties of Soils and Shallow Sediments at the Savannah River Site (U), WSRC-RP-90-1031, Westinghouse Savannah River Company, Aiken, $\mathrm{SC}$.

Oma, K.H., 1994. In Situ Vitrification, Hazardous Waste Site Soil Remediation - Theory and Application of Innovative Technologies, Wilson, D.J. and Clarke, A.N., eds., Marcel Dekker, Inc., New York, New York.

Tixier, J.S., 1995. Personal communication between Pacific Northwest Laboratories and RUST E\&I on February 1, 1995.

USDA (U.S. Department of Agriculture), 1990. Soil Survey of Savannah River Plant Area, Parts of Aiken, Barnwell, and Allendale Counties, South Carolina, U.S. Department of Agriculture, Soil Conservation Service.

Warren, R.W., et.al., 1994. Water Balance Relationships In Four Alternative Cover Designs For Radioactive And Mixed Waste Landfills; prepared for the USDOE Office of Technology Development/Office of Environmental Management and submitted to the American Nuclear Society for publication. 
WSRC (Westinghouse Savannah River Company), 1989. RCRA Facility Investigation Program Plan, WSRC-RP-89-994, Westinghouse Savannah River Company, Savannah River Site, Aiken, SC.

WSRC (Westinghouse Savannah River Company), 1993a. Federal Facility Agreement for the Savannah River Site, Appendix C. Docket No. 89-05-FF, WSRC-RP-94-42, Westinghouse Savannah River Company, Savannah River Site, Aiken, SC.

WSRC (Westinghouse Savannah River Company), 1993b. RCRA Facility Investigation/RI Program Plan, WSRC-RP-89-994, Revision 1, Westinghouse Savannah River Company, Savannah River Site, Aiken, SC.

* WSRC (Westinghouse Savannah River Company), 1993c. Phase II, Revision 2, RCRA Facility Investigation/Remedial Investigation Plan for the L-Area Oil and Chemical Basin (904-83G) (U), WSRC-RP-90-1028, Rev. 2. Westinghouse Savannah River Company, Savannah River Site, Aiken, SC.

WSRC (Westinghouse Savannah River Company), 1994. Savannah River Site Environmental Report for 1993, WSRC-TR-94-075, Environmental Monitoring Section-Environmental Protection Department; Westinghouse Savannah River Company, Savannah River Site, Aiken, SC.

*WSRC (Westinghouse Savannah River Company), 1995a. Radionuclide Background Levels in Production of Unimpacted SRS Soils Versus Preliminary Remediation Goals (U), Q-ESR-G-00011, Westinghouse Savannah River Company, Aiken, SC.

WSRC (Westinghouse Savannah River Company), 1995b. Savannah Rive Site Waste Acceptance Criteria Manual, Manual 1S, Westinghouse Savannah Rive Company, Aiken, $\mathrm{SC}$.

WSRC (Westinghouse Savannah River Company), 1996a. RCRA Facility Investigation/ Remedial Investigation Report for the L-Area Oil and Chemical Basin, WSRC-RP-95305, Revision 0. Westinghouse Savannah River Company, Savannah River Site, Aiken, $\mathrm{SC}$.

*WSRC (Westinghouse Savannah River Company), 1996b. Baseline Risk Assessment for the L-Area Oil and Chemical Basin, WSRC-RP-95-387, Revision 0. Westinghouse Savannah River Company, Savannah River Site, Aiken, SC.

* WSRC (Westinghouse Savannah River Company), 1996c. Laboratory-Scale Immobilization Study Report for the L-Area Oil and Chemical Basin, WSRC-RP-96-15,-Revision 0. Westinghouse Savannah River Company, Savannah River Site, Aiken, SC. 
003664

Appendix A

Dose Rate Calculations 
PHASE I FOCUSED CMS/FS REPORT

L-AREA OIL AND CHEMICAL BASIN
WSRC-RP-96-106, REV. 1.1

FEBRUARY 1997

THIS PAGE INTENTIONALLY LEFT BLANK 

REDUCTIONS DUE TO RADIOACTIVE DECAY AND SHIELDING

Cesium-137 Concentration and Effective Dose Equivalent Reduction from Radioactive Decay and Capping

Based on the Maximum Concentration of Cesium-137

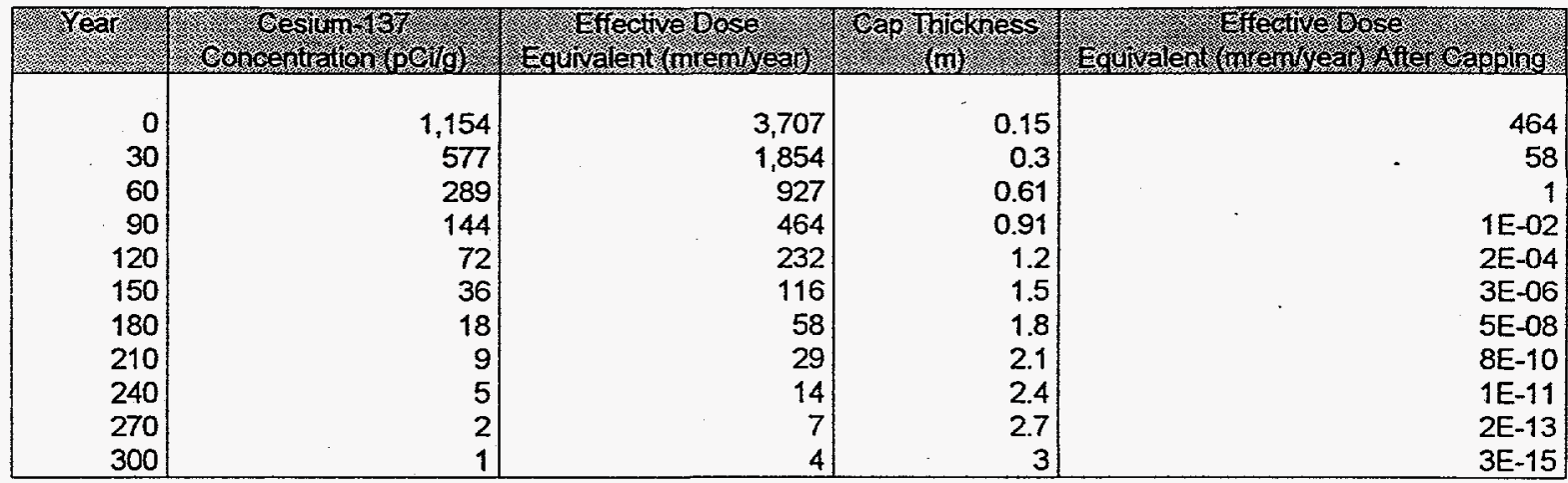

Cobalt-60 Concentration and Effective Dose Equivalent Reduction from Radioactive Decay and Capping

Based on the Maximum Concentration of Cobalt -60

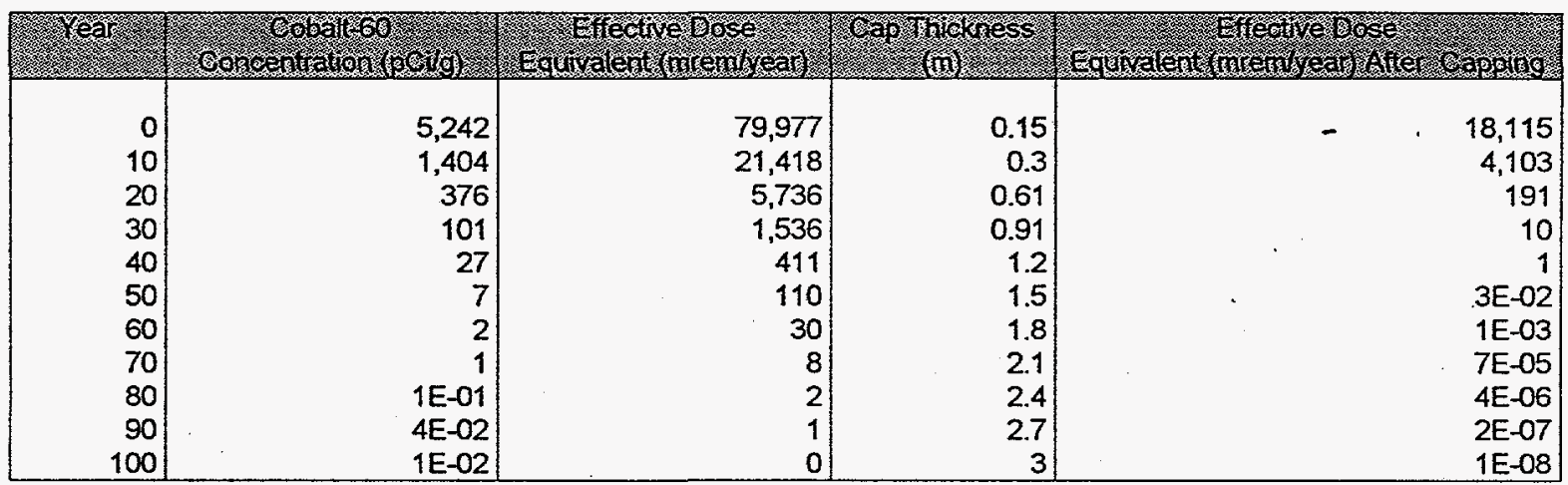

Dose Conversion Factor for Cesium-137 (Barium-137 X-ray) $=8.8 \mathrm{E}-03 \mathrm{mrem} /$ day/pCi/g Dose Conversion Factor for Cobalt $-60=4.2 E-02 \mathrm{mrem} / \mathrm{day} / \mathrm{pCi} / \mathrm{g}$

Reference: NRC (Nuclear Regulatory Commission), 1992. Residual Radioactive Contamination From Decommissioning, Final Report, NUREG/CR-5512, PNL-7994, Vol.1.

Mass Attenuation Coefficient per Unit Density (soii) for Cesium-137 (0.662 MeV gamma) = 7.7E-06 m2/g Mass Attenuation Coefficient per Unit Density (soil) for Cobalt-60 (1.332 MeV gamma) $=5.5 E-06 \mathrm{~m} 2 / \mathrm{g}$

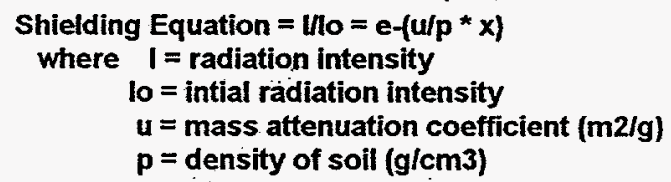

Reference: Turner, J.E. 1986. Atoms, Radiation, and Radiation Protection, Pergammon Press, New York, New York 
THIS PAGE INTENTIONALLY LEFT BLANK 
Appendix B

Cost Estimates 


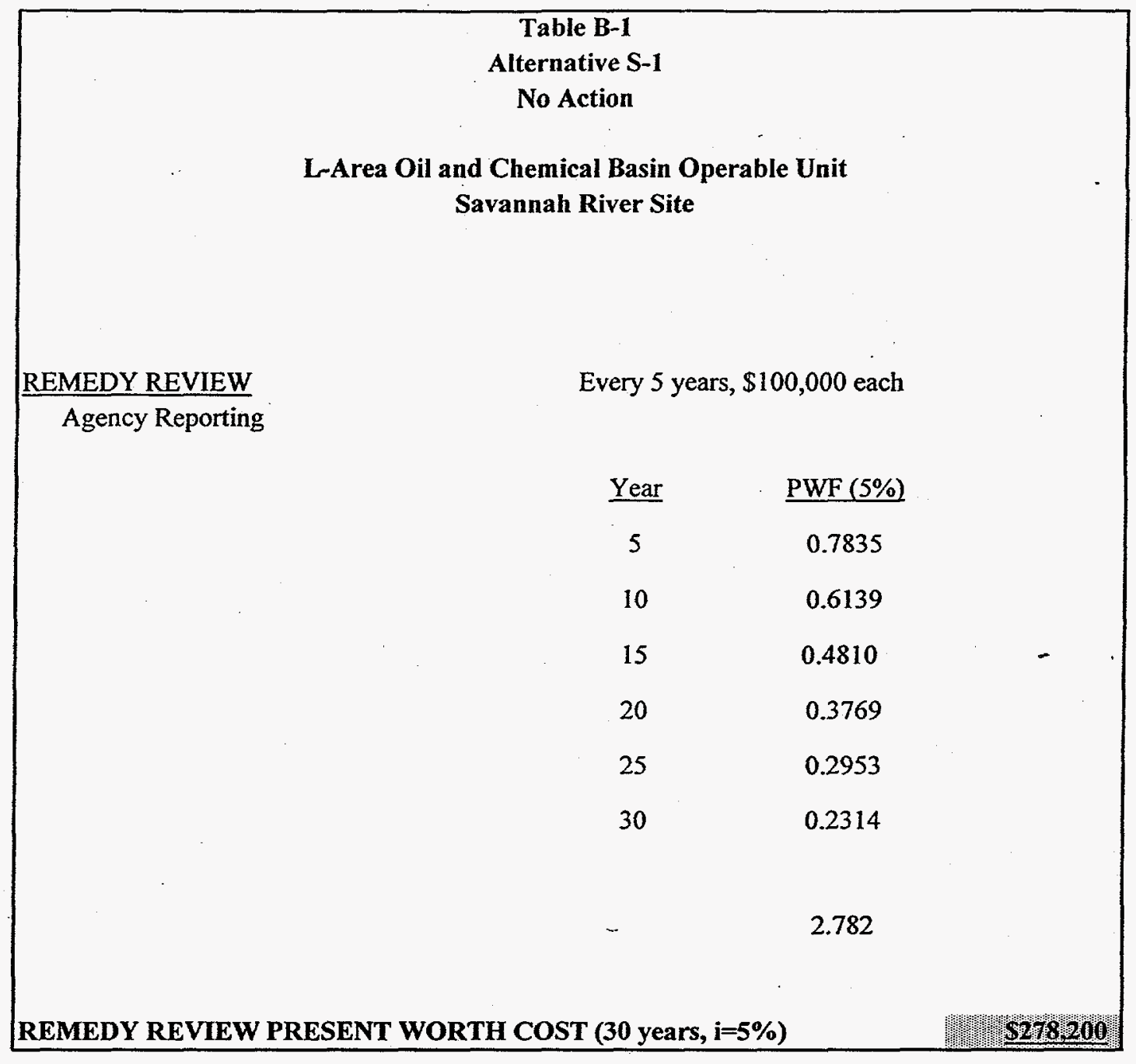




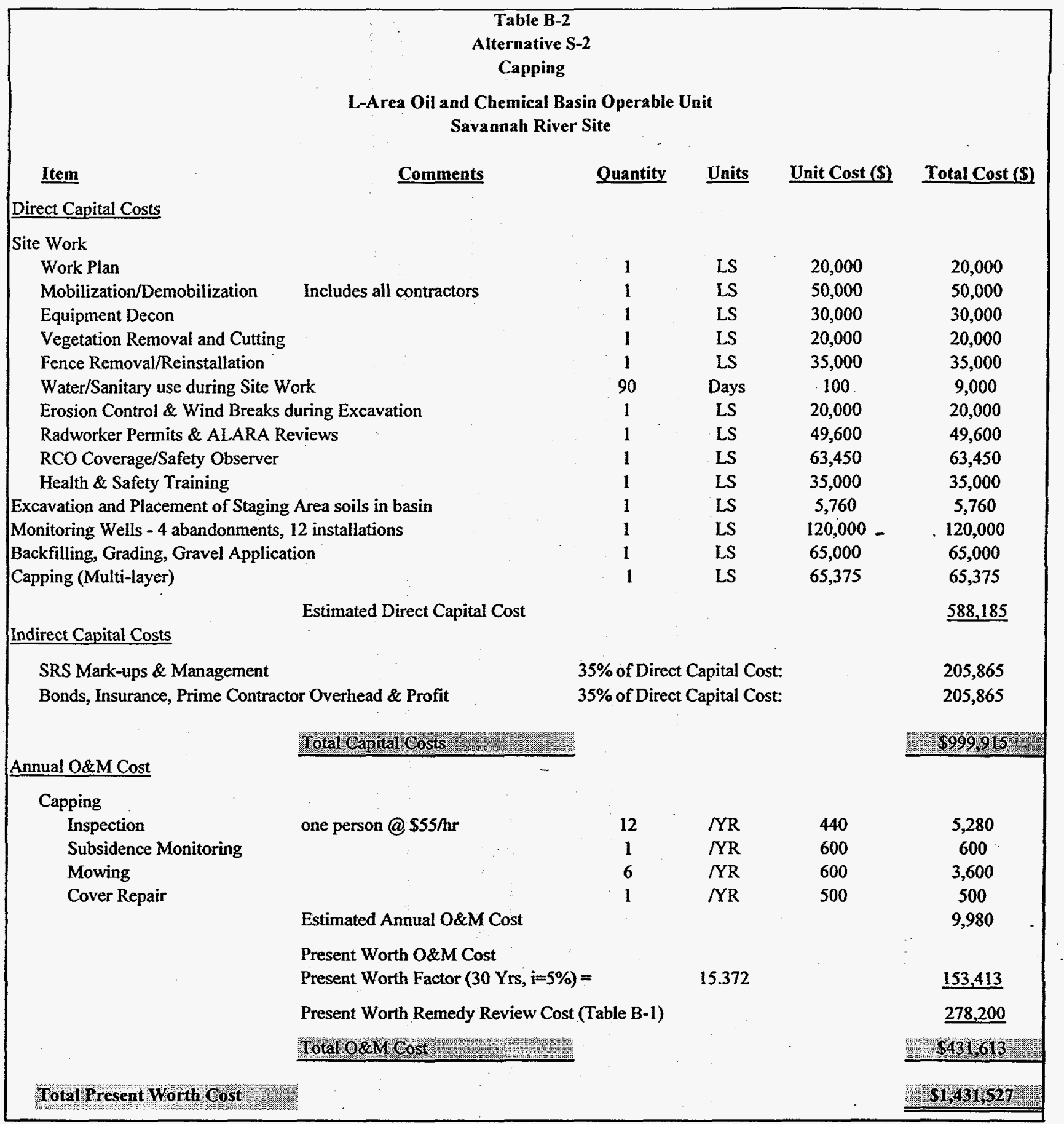




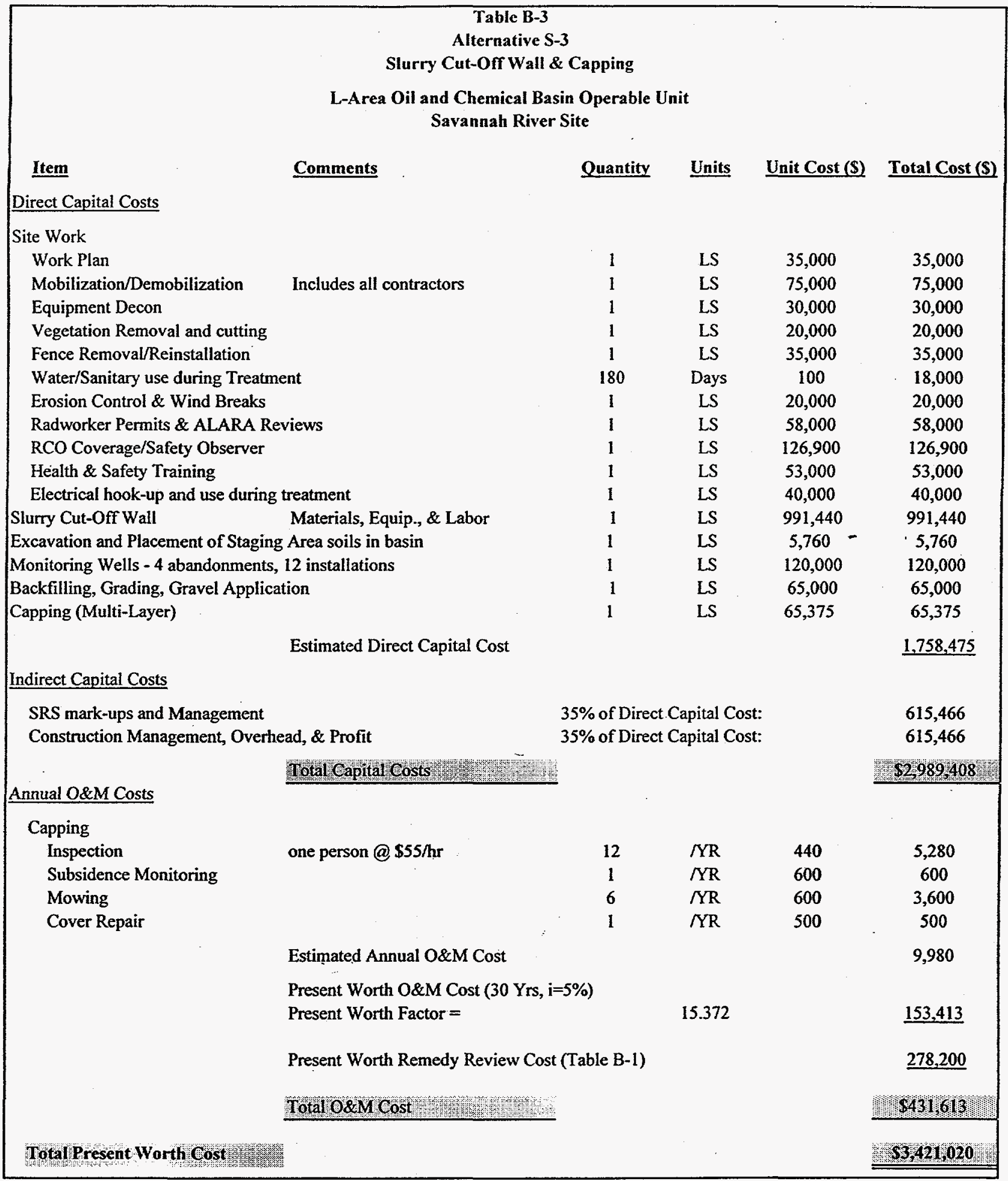




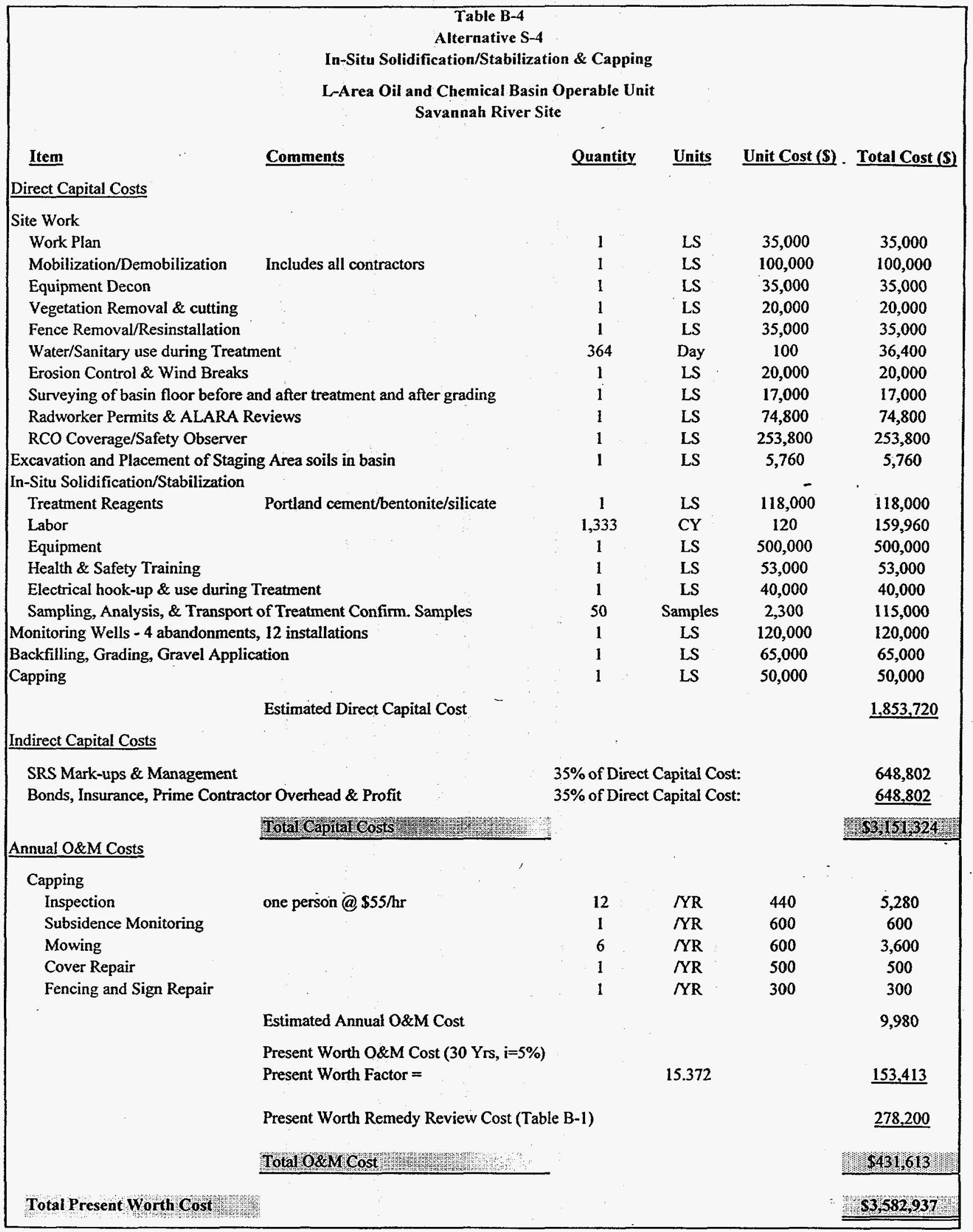




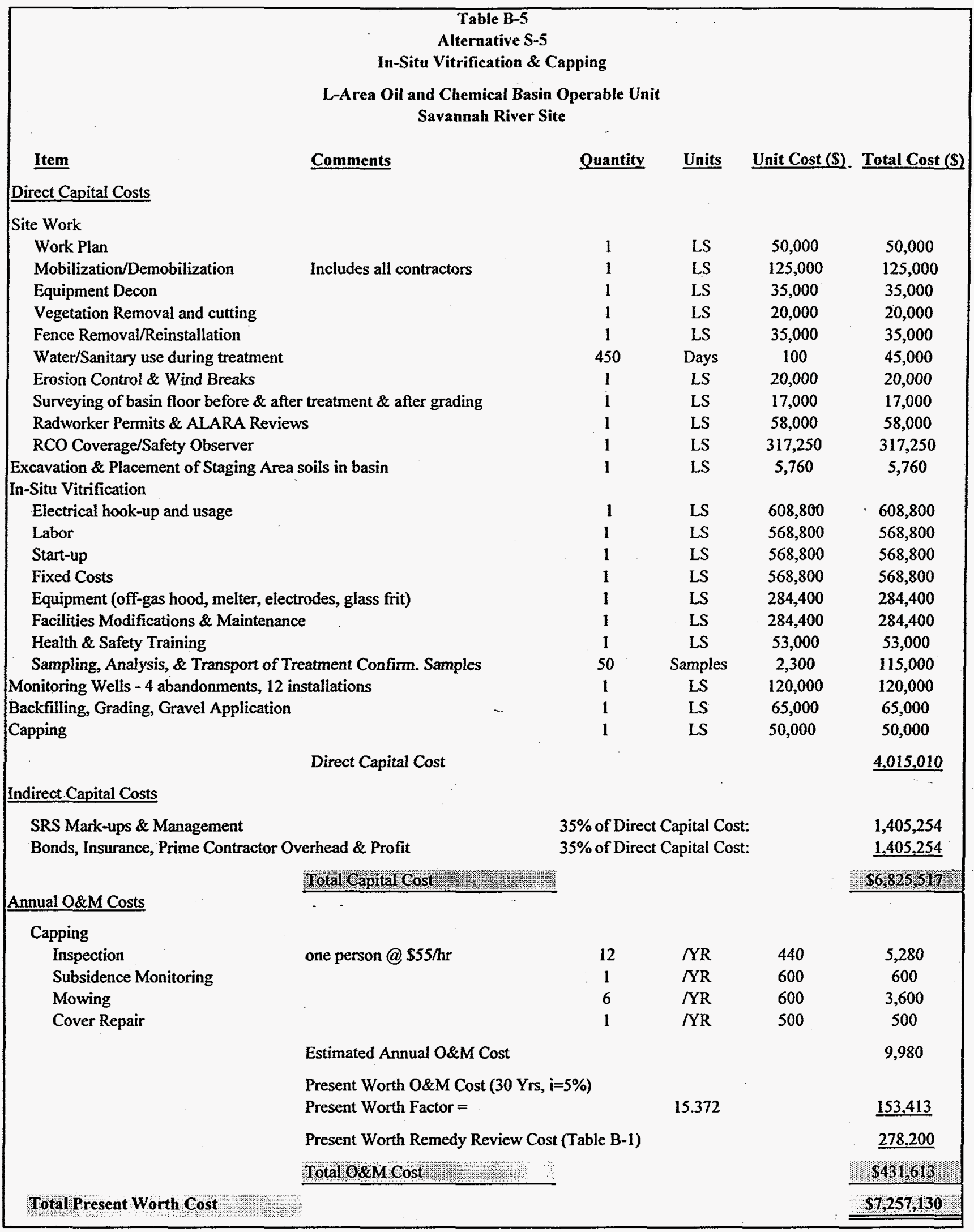




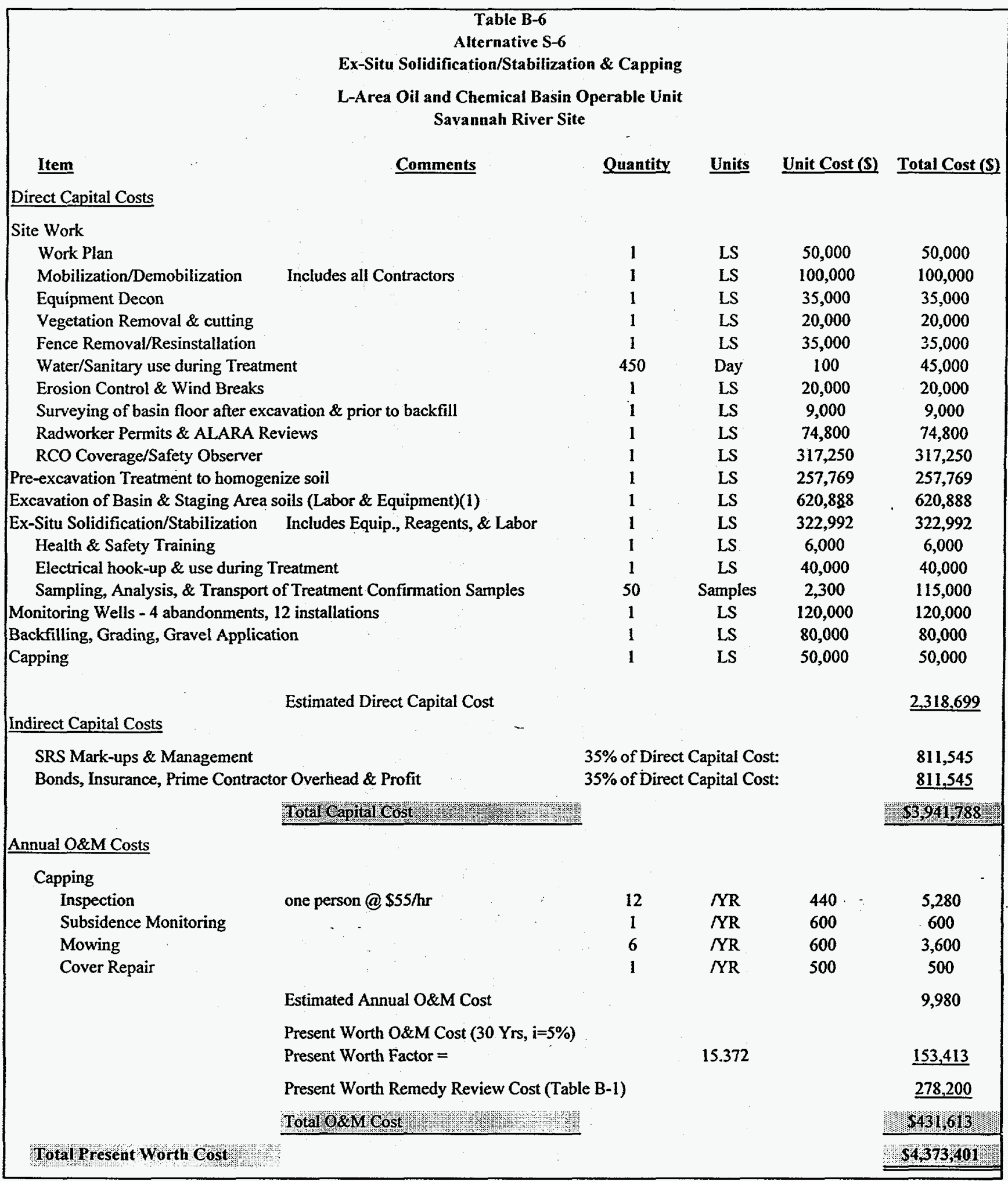

(1) Equipment will be decomissioned following excavation of soils due to high radiation exposure. 


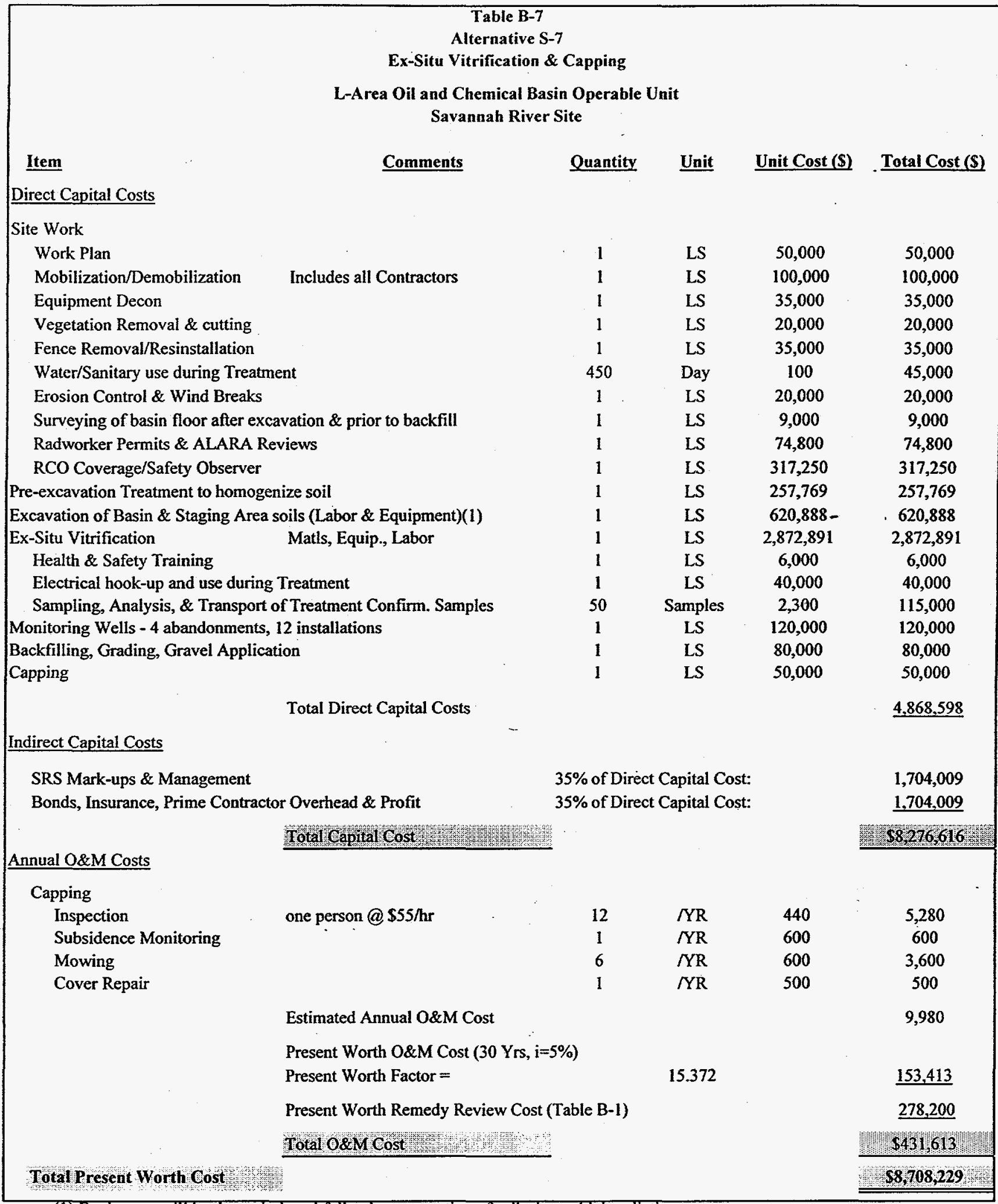

(1) Equipment will be decomissioned following excavation of soils due to high radiation exposure. 


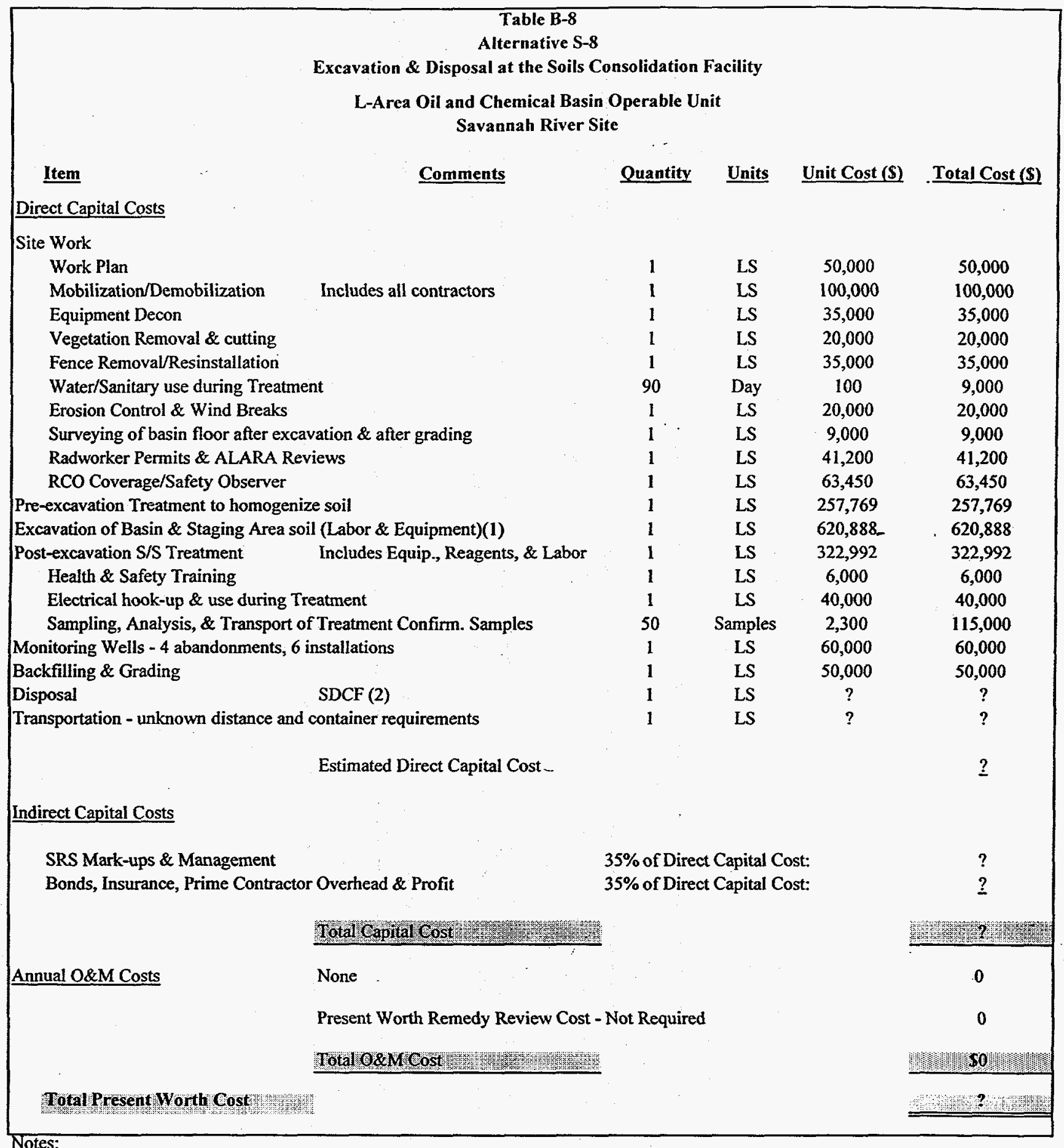

(1) Equipment will be decomissioned following use due to high radiation exposure.

(2) Disposal cost is unknown because the Soils Consolidation Facility has not yet been sited, designed, or constructed. 


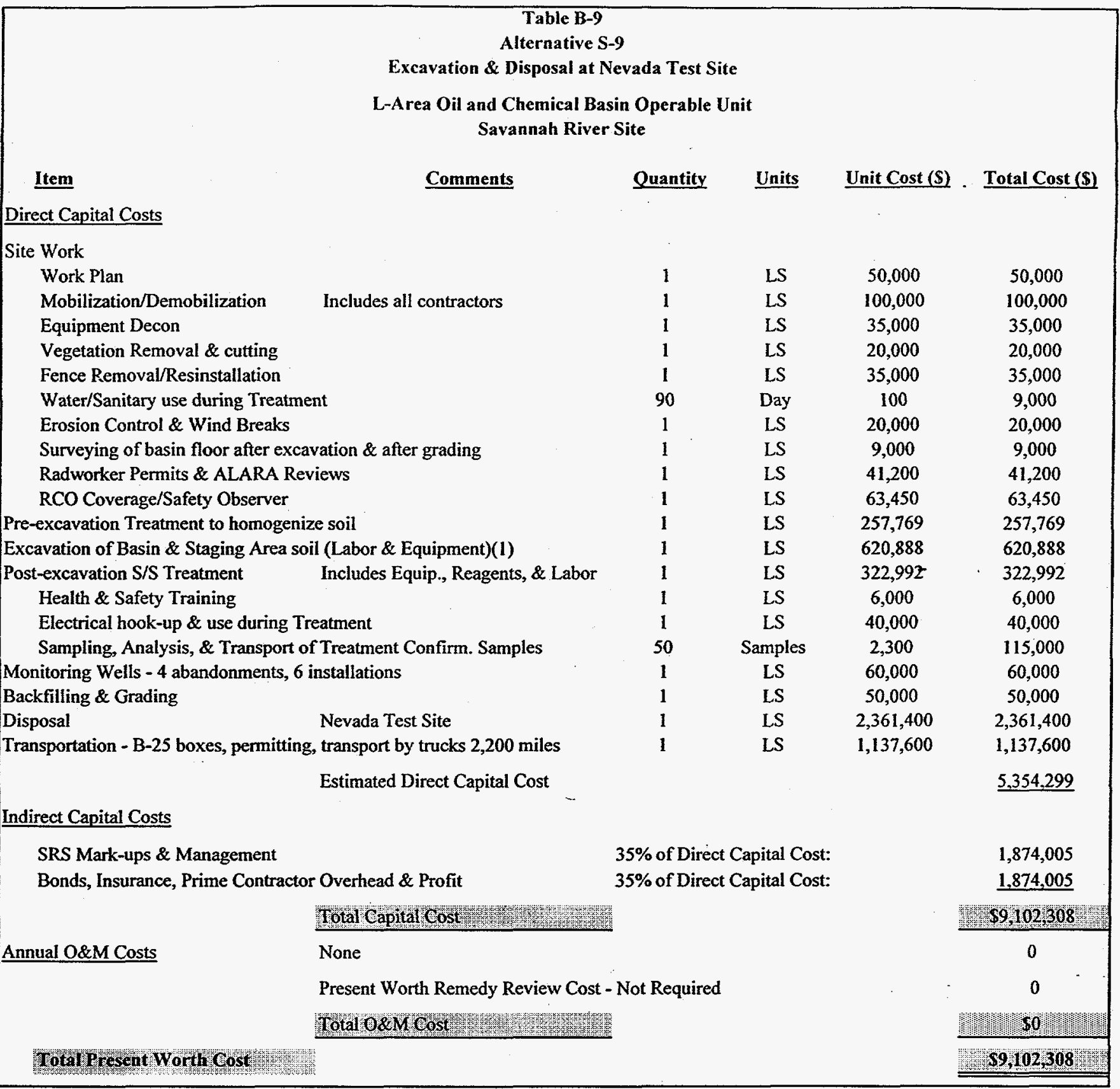

(1) Equipment will be decomissioned following excavation of soil due to high radiation exposure 


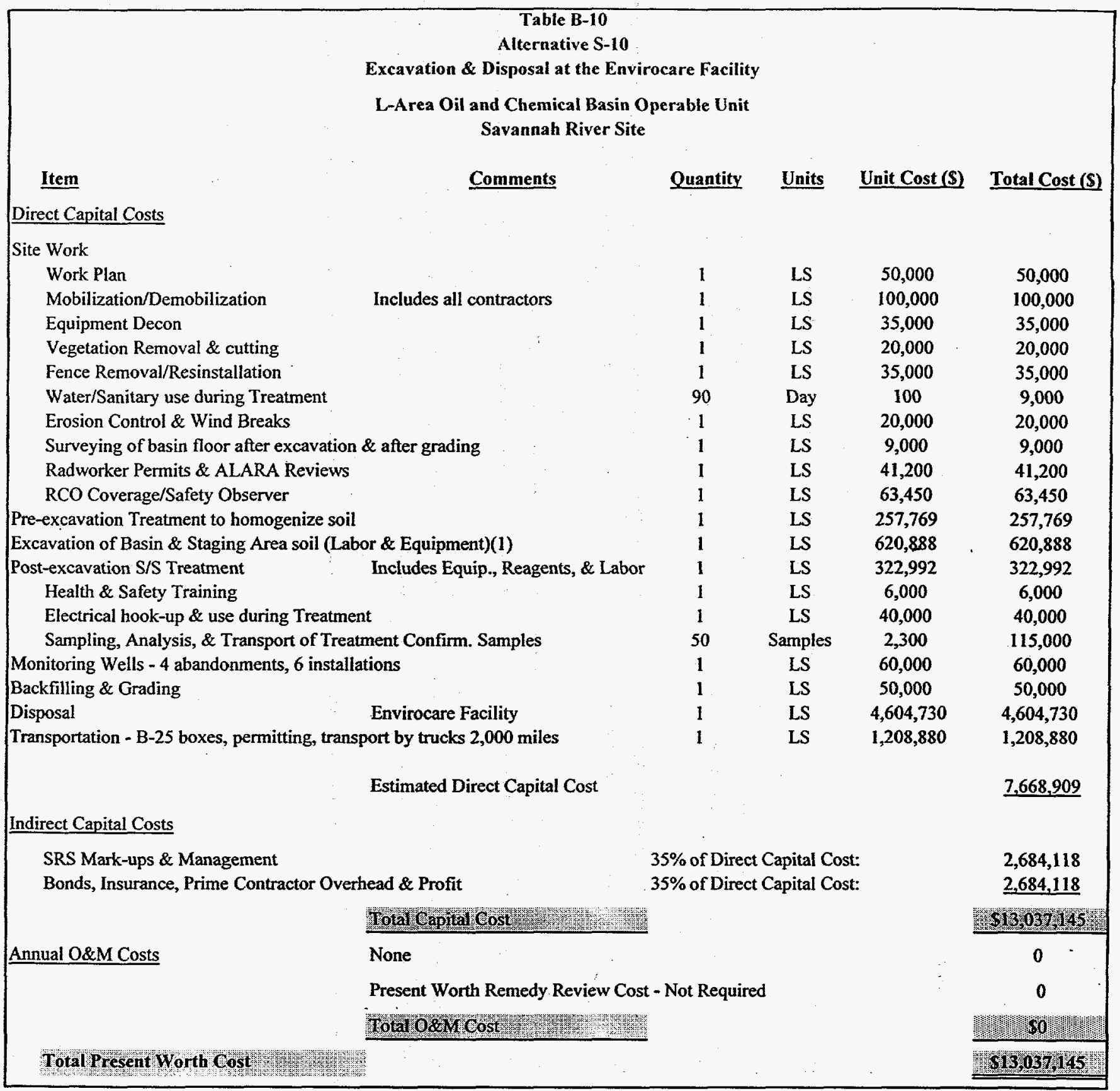




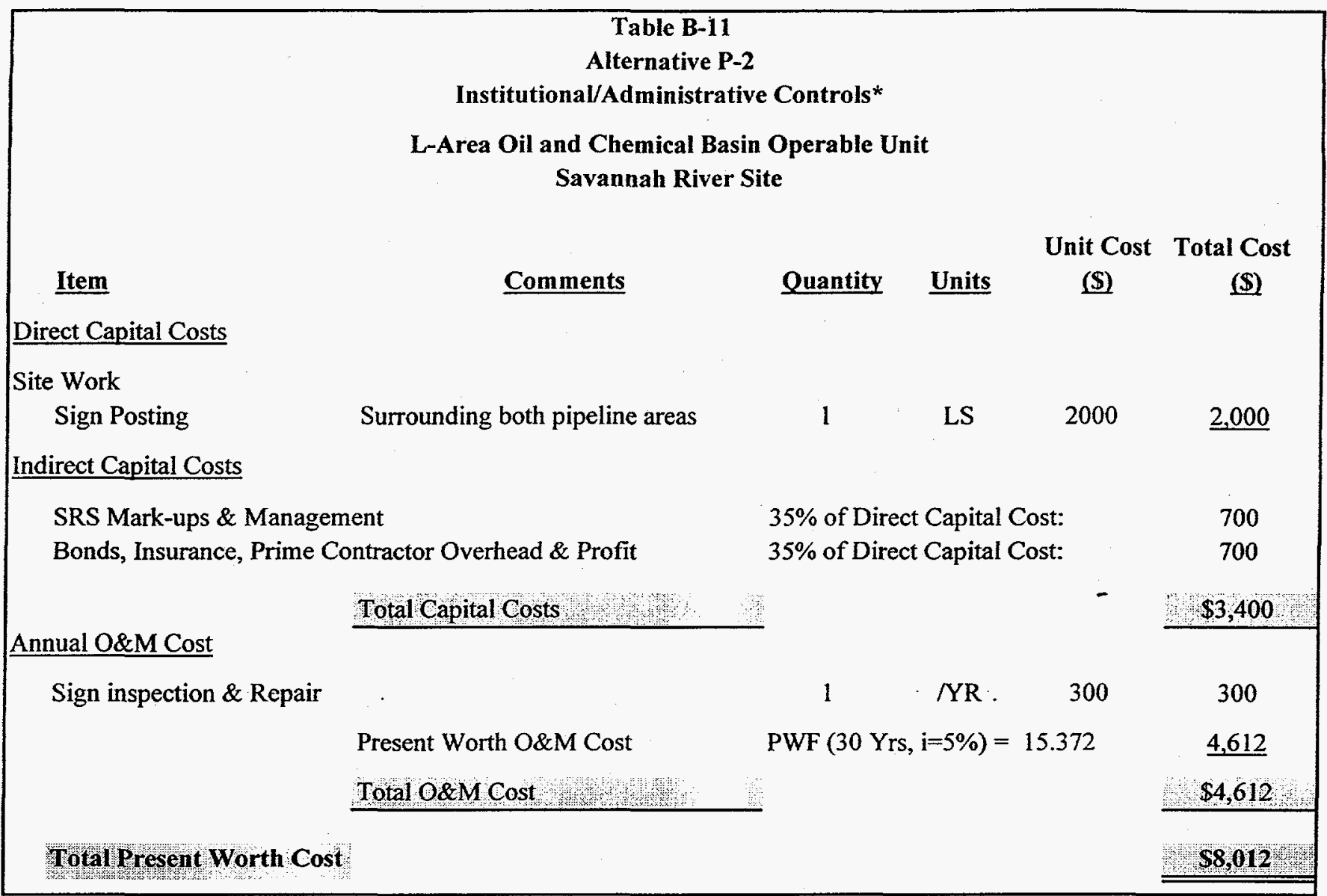

* Periodic Monitoring and 5-year Remedy Reviews are assumed to be covered by the LAOCB remedy. 


\begin{tabular}{|c|c|c|c|c|c|}
\hline & $\begin{array}{r}\text { Table } \\
\text { Alternat } \\
\text { Cappi } \\
\text { L-Area Oil and Chemica } \\
\text { Savannah }\end{array}$ & $\begin{array}{l}\text { Operable } \\
\text { ite }\end{array}$ & nit & & \\
\hline$\underline{\text { Item }}$ & Comments & Quantity & Units & (\$) & $\begin{array}{l}\text { Total Cost } \\
\text { (\$) }\end{array}$ \\
\hline Direct Capital Costs & & & & & \\
\hline Site Work & & & & & \\
\hline Work Plan & & 1 & LS & 20,000 & 20,000 \\
\hline Mobilization/Demobilization & Includes all contractors & 1 & LS & 50,000 & 50,000 \\
\hline Equipment Decon & & 1 & LS & 30,000 & 30,000 \\
\hline Water/Sanitary use during Site & Nork & 60 & Days & 100 & 6,000 \\
\hline Radworker Permits \& ALARA & Reviews & 1 & LS & 32,800 & 32,800 \\
\hline RCO Coverage/Safety Observ & & 1 & LS & 42,300 & 42,300 \\
\hline Health \& Safety Training & & 1 & LS & 35,000 & 35,000 \\
\hline Fence Installation & & 1 & LS & $40,0 \overline{0} 0$ & 40,000 \\
\hline Sign Posting & & 1 & LS & 2,000 & 2,000 \\
\hline Capping (Multi-layer) & & 1 & LS & 69,014 & 69,014 \\
\hline Indirect Capital Costs & Estimated Direct Capital & & & & $\underline{327,114}$ \\
\hline $\begin{array}{l}\text { SRS Mark-ups \& Management } \\
\text { Bonds, Insurance, Prime Contr }\end{array}$ & tor Overhead \& Profit & $\begin{array}{l}35 \% \text { of Dir } \\
35 \% \text { of Dir }\end{array}$ & $\begin{array}{l}\text { Capital } \\
\text { Capital }\end{array}$ & $\begin{array}{l}\text { ost: } \\
\text { ost: }\end{array}$ & $\begin{array}{l}114,490 \\
114,490\end{array}$ \\
\hline & Total Capital Costs & & & & $\$ 556,094$ \\
\hline Annual O\&M Cost & & & & & \\
\hline Capping & & & & & \\
\hline Inspection & one person@ @ \$55/hr & 12 & /YR & $440^{\circ}$ & 5,280 \\
\hline Subsidence Monitoring & & 1 & MR & 600 & 600 \\
\hline Mowing & & 6 & NRR & 600 & 3,600 \\
\hline Cover Repair & & 1 & /Y R & 500 & 500 \\
\hline Sign inspection \& repair & & 1 & rR & 300 & $\underline{300}$ \\
\hline & Estimated Annual O\&M & & & & 10,280 \\
\hline & Present Worth O\&M Cos & & & & \\
\hline & Present Worth Factor $(30$ & $=5 \%)=$ & 15.372 & & $\underline{158,024}$ \\
\hline & Total O\&M Cost & & & & $\$ 168,304$ \\
\hline Total Present Worth Cost & & & & & $\$ 724,398$ \\
\hline
\end{tabular}

* Periodic Monitoring and 5-year Remedy Reviews are assumed to be covered by the LAOCB remedy. 


\begin{tabular}{|c|c|c|c|c|c|}
\hline & $\begin{array}{r}\text { Tabl } \\
\text { Alterna } \\
\text { In-Situ Sta } \\
\text { L-Area Oil and Chemi } \\
\text { Savannah }\end{array}$ & $\begin{array}{l}4 \\
\text { ion* } \\
\text { in Operable } \\
\text { Site }\end{array}$ & & & \\
\hline$\underline{\text { Item }}$ & Comments & Quantity & $\underline{\text { Units }}$ & $\begin{array}{c}\text { Unit Cost } \\
\text { (\$) }\end{array}$ & $\begin{array}{c}\text { Total Cost } \\
\text { (\$) }\end{array}$ \\
\hline Direct Capital Costs & & & & & \\
\hline Site Work & & & & & \\
\hline Work Plan & & 1 & LS & 20,000 & 20,000 \\
\hline Mobilization/Demobilization & Includes all contractors & 1 & LS & 25,000 & 25,000 \\
\hline Equipment Decon & & 1 & LS & 10,000 & 10,000 \\
\hline Water/Sanitary use during trea & nent & 10 & Days & 100 & 1,000 \\
\hline Radworker Permits \& ALARA & Reviews & 1 & LS & 32,800 & 32,800 \\
\hline RCO Coverage/Safety Observe & & 1 & LS & 6,953 & 6,953 \\
\hline Health \& Safety for Treatment & & 1 & LS & 5,000 & 5,000 \\
\hline In-Situ Solidification & Equip., Matls, \& Labor & 1 & LS & 20,000 & 20,000 \\
\hline Fence Installation & & 1 & LS & 40,000 & 40,000 \\
\hline Sign Posting & & 1 & LS & 2,000 & 2,000 \\
\hline & Estimated Direct Capital & & & & $\underline{162,753}$ \\
\hline Indirect Capital Costs & & & & & \\
\hline SRS Mark-ups \& Management & & $35 \%$ of Dire & Capital & & 56,964 \\
\hline Bonds, Insurance, Prime Contr & tor Overhead \& Profit & $35 \%$ of Dire & Capital & & 56,964 \\
\hline & Total Capital Costs & & & & $\$ 276,681$ \\
\hline Annual O\&M Costs & & & & & \\
\hline Sign inspection \& repair & & 1 & $/ \mathrm{YR}$ & 300 & 300 \\
\hline & Present Worth O\&M Cos & $\mathrm{rs}, \mathrm{i}=5 \%, \mathrm{PY}$ & $=15.372$ & & $\underline{4,612}$ \\
\hline & Total O\&U Cost & & & & (\$ $84,612 m$ \\
\hline Total Present Worth Cost & & & & & $\$ 281,292$ \\
\hline
\end{tabular}

* Periodic Monitoring and 5-year Remedy Reviews are assumed to be covered by the LAOCB remedy. 


\begin{tabular}{|c|c|c|c|c|c|}
\hline & $\begin{array}{r}\text { Table B } \\
\text { Alternati } \\
\text { High Pressure Wash } \\
\text { L-Area Oil and Chemical } \\
\text { Savannah R }\end{array}$ & $\begin{array}{l}\text { [n-Situ } S / S^{*} \\
\text { Operable } \\
\text { ite }\end{array}$ & & & \\
\hline$\underline{\text { Item }}$ & Comments & Quantity & $\underline{\text { Units }}$ & $\begin{array}{c}\text { Unit Cost } \\
\text { (\$) }\end{array}$ & $\begin{array}{c}\text { Total Cost } \\
(\$)\end{array}$ \\
\hline Direct Capital Costs & & & & & \\
\hline Site Work & & & & & \\
\hline Work Plan & & 1 & LS & 30,000 & 30,000 \\
\hline Mobilization/Demobilization & Includes all contractors & 1 & LS & 35,000 & 35,000 \\
\hline Equipment Decon & & 1 & LS & 30,000 & 30,000 \\
\hline Water/Sanitary use during trea & & 15 & Days & 100 & 1,500 \\
\hline Radworker Permits \& ALARA & eviews & 1 & LS & 41,200 & 41,200 \\
\hline RCO Coverage/Safety Observ & & 1 & LS & 10,430 & 10,430 \\
\hline Health \& Safety for Treatment & & 1 & LS & 10,000 & 10,000 \\
\hline High Pressure Washing & Labor \& Materials & 1 & LS & 15,000 & 15,000 \\
\hline In-Situ Solidification & Equip., Matls, \& Labor & 1 & LS & 20,000 & 20,000 \\
\hline Fence Installation & & 1 & LS & 40,000 & 40,000 \\
\hline Sign Posting & & 1 & LS & 2,000 & 2,000 \\
\hline & Estimated Direct Capital & & & & $\underline{235,130}$ \\
\hline$\underline{\text { Indirect Capital Costs }}$ & & & & & \\
\hline SRS Mark-ups \& Management & & $35 \%$ of Dire & t Capital & & 82,296 \\
\hline Bonds, Insurance, Prime Contr & or Overhead \& Profit & $35 \%$ of Dire & t Capital & & 82,296 \\
\hline ... & Total Capital Costs & & - & & $\$ 399,721$ \\
\hline Annual O\&M Costs & & & & & \\
\hline Sign inspection \& repair & & 1 & /YR & 300 & 300 \\
\hline & Present Worth O\&M Cos & Yrs, $\mathrm{i}=5 \%)$ & & & \\
\hline & Present Worth Factor = & & 15.372 & & $\underline{4,612}$ \\
\hline & Total O\&M Cost & & & & W 84.612 \\
\hline Total Present Worth Cost & & & & & $\$ 404,333$ \\
\hline
\end{tabular}

* Periodic Monitoring and 5-year Remedy Reviews are assumed to be covered by the LAOCB remedy. 


\begin{tabular}{|c|c|c|c|c|c|}
\hline & $\begin{array}{r}\text { Tabl } \\
\text { Altern } \\
\text { In Situ Stabilization an } \\
\text { L-Area Oil and Chemi } \\
\text { Savannah }\end{array}$ & $\begin{array}{l}\text { in the } \mathrm{LAC} \\
\text { Operable } \mathrm{C} \\
\text { ite }\end{array}$ & $\begin{array}{l}\mathrm{CB} \\
\text { nit }\end{array}$ & & \\
\hline Item & Comments & Quantity & Units & $\begin{array}{c}\text { Unit Cost } \\
\text { (\$) }\end{array}$ & $\begin{array}{c}\text { Total Cost } \\
\text { (\$) }\end{array}$ \\
\hline Direct Capital Costs & & & & & \\
\hline Site Work & & & & & \\
\hline Work Plan & & 1 & LS & 30,000 & 30,000 \\
\hline Mobilization/Demobilization & Includes all contractors & 1 & LS & 55,000 & 55,000 \\
\hline Equipment Decon & & 1 & LS & 30,000 & 30,000 \\
\hline Water/Sanitary use during trea & ment/excavation & 60 & Days & 100 & 6,000 \\
\hline Radworker Permits \& ALARA & Reviews & 1 & LS & 58,000 & 58,000 \\
\hline RCO Coverage/Safety Observ & & 1 & LS & 42,300 & 42,300 \\
\hline Health \& Safety for Treatment & \& Excavation & 1 & LS & 53,000 & 53,000 \\
\hline In-Situ Solidification & Equip., Matls, \& Labor & 1 & LS & 20,000 & 20,000 \\
\hline Excavation \& Cutting of Pipeline 8 & Associated Soil & 1 & LS & 216,000 & 216,000 \\
\hline Backfilling \& Grading Pipeline Ar & & 1 & LS & 50,000 & 50,000 \\
\hline Confirmation Sampling/Analyses/T & ansportation & 60 & Samples & 400 & 24,000 \\
\hline & Estimated Direct Capita & & & & $\underline{584,300}$ \\
\hline Indirect Capital Costs & & & & & \\
\hline SRS Mark-ups \& Managemen & & $35 \%$ of Dire & et Capital C & & 204,505 \\
\hline Bonds, Insurance, Prime Contr & ctor Overhead \& Profit & $35 \%$ of Dire & ct Capital C & & 204,505 \\
\hline & Total Capital Costs & & & & $\$ 993,310$ \\
\hline Annual O\&M Costs & None & & & & 0 \\
\hline & Present Worth Remedy & Cost - Not Re & quired & & 0 \\
\hline & Total O\&MCost & & & & 80. \\
\hline Total Present Worth Cost & & & & & $\$ 993310$ \\
\hline
\end{tabular}

This alternative only includes excavating and delivering the pipelines to the LAOCB. Costs for subsequent treatment or disposal of the pipelines as part of the LAOCB is not included. 


\begin{tabular}{|c|c|c|c|c|c|}
\hline & $\begin{array}{r}\text { Table } \\
\text { Alterna } \\
\text { Situ Stabilization and D } \\
\text { L-Area Oil and Chemi } \\
\text { Savannah }\end{array}$ & $\begin{array}{l}\text { t Nevada T } \\
\text { Operable } \mathrm{C} \\
\text { te }\end{array}$ & th Site & & \\
\hline$\underline{\text { Item }}$ & Comments & Quantity & Units & $\begin{array}{l}\text { Unit Cost } \\
\qquad(\$)\end{array}$ & $\begin{array}{c}\text { Total Cost } \\
\text { (\$) }\end{array}$ \\
\hline Direct Capital Costs & & & & & \\
\hline Site Work & & & & & \\
\hline Work Plan & & 1 & LS & 30,000 & 30,000 \\
\hline Mobilization/Demobilization & Includes all contractors & 1 & LS & 55,000 & 55,000 \\
\hline Equipment Decon & & 1 & LS & 30,000 & 30,000 \\
\hline Water/Sanitary use during trea & ient/excavation & 90 & Days & 100 & 9,000 \\
\hline Radworker Permits \& ALAR & Reviews & 1 & LS & 58,000 & 58,000 \\
\hline RCO Coverage/Safety Observ & & 1 & LS & 63,450 & 63,450 \\
\hline Health \& Safety for Treatmen & Excavation & 1 & LS & $53, \overline{0} 00$ & 53,000 \\
\hline In-Situ Solidification & Equip., Matls, \& Labor & 1 & LS & 20,000 & 20,000 \\
\hline Excavation \& Cutting of Pipeline & Associated Soil & 1 & LS & 216,000 & 216,000 \\
\hline Backfilling \& Grading Pipeline Ar & & 1 & LS & 50,000 & 50,000 \\
\hline Confirmation Sampling/Analyses/ & insportation & 60 & Samples & 400 & 24,000 \\
\hline Packaging Pipelines for Transporta & & 1 & LS & 50,000 & 50,000 \\
\hline Roll-off Boxes for transport & & 2 & Boxes & 2,000 & 4,000 \\
\hline Disposal of pipelines in boxes & Nevada Test Site & 1 & LS & $1,393,000$ & $1,393,000$ \\
\hline Transportation & 2,200 miles & 1 & LS & 670,704 & 670,704 \\
\hline & Estimated Direct Capital & & & & $\underline{2,726,154}$ \\
\hline Indirect Capital Costs & & & & & \\
\hline SRS Mark-ups \& Managemen & & $35 \%$ of Dire & t Capital & & 954,154 \\
\hline Bonds, Insurance, Prime Contr & tor Overhead \& Profit & $35 \%$ of Dire & Capital & ost: & 954,154 \\
\hline & Total Capital Cost & & & & W4,634,462 \\
\hline Annual O\&M Costs & None & & & & 0 \\
\hline & Present Worth Remedy $\mathrm{F}$ & ost - Not Re & uired & & 0 \\
\hline & Total O\&M Cost & & & & 80 \\
\hline Total Present Worth Cost & & & & & $\$ 4,634,462$ \\
\hline
\end{tabular}




$$
3664 e
$$

